AAAAAAC

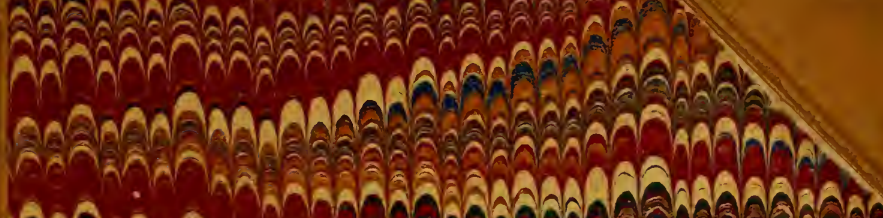

AnAिA

ÂैA

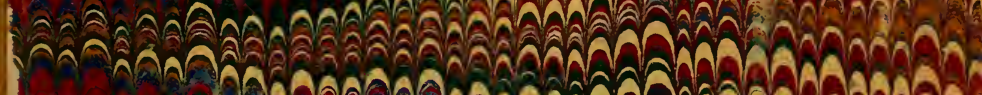

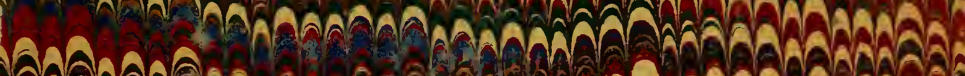
¿̂ै-

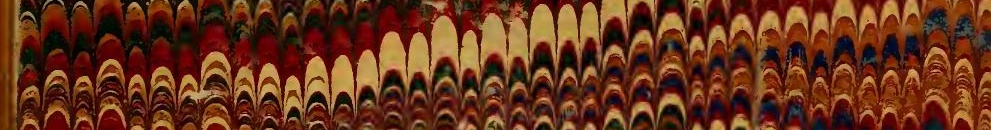

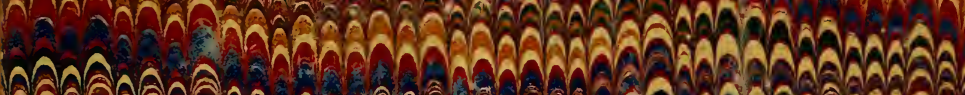

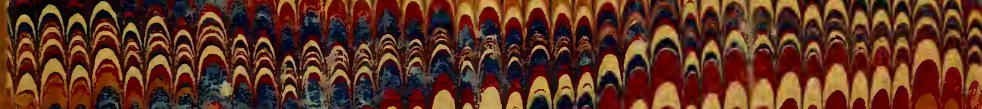

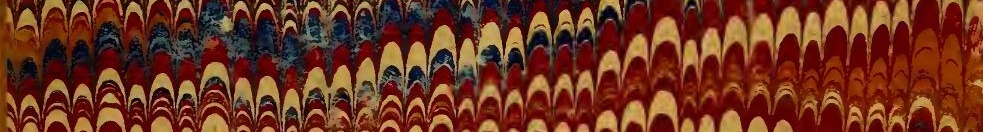
1

1. A A

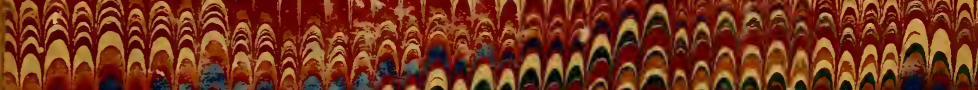

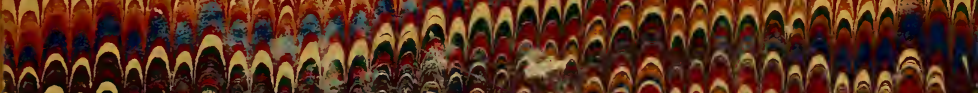
HADAOHS Y An

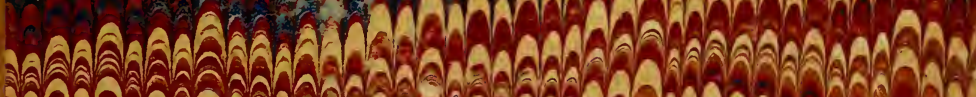
MAn

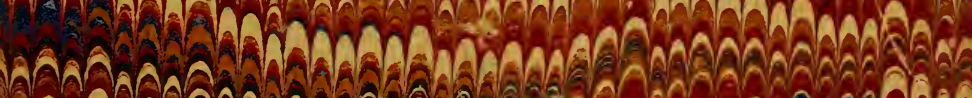
MAn

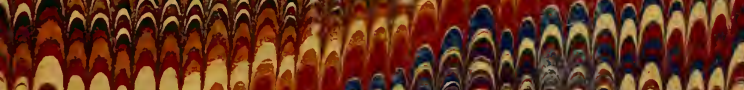
A (n)

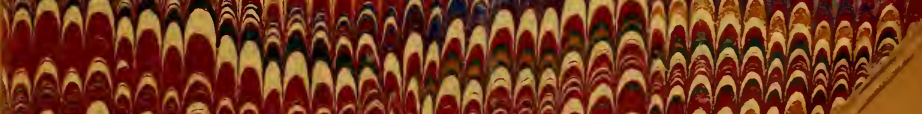
AnA anA ลวกลล 



14840

Ahe Ayaseis Caculiify 1869 



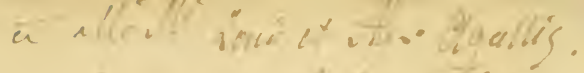

14846

\section{VOYA G E}

A UX

\section{L E S B A L É A R E S}


$\therefore$

$: \because$

PARIS. - IMPRIMERIE DE L. MARTINET, RUE MIGNON, 2. 


\title{
VOYA GE
}

\section{A U X \\ ILES BALEARES \\ ou. \\ R E C H E R C H E S}

SUR l'ANATOMIE ET LA PHYSIOLOGIE DE QUELQUES MOLLUSQUES

\section{DE LA MÉDITERRANÉE}

\author{
PAR LE DOCTEUR \\ F. - J. - H. LACAZE - DUTHIERS \\ Professeur à la Faculté des sciences de Lille \\ Membre des Sociétés philomatique de Paris et des sciences de Lillo
}

\section{PARIS}

L.IBRAIRIE DE VICTOR MASSON

PLACE DE L'ÉCOLL-DE-MÉdECINE 

A

\section{J U L ES H A I M E.}

AMI,

Je te dédie ces pages en souvenir de notre voyage.

Puisse ce gage de l'amitié te faire oublier un instant tes longues souffrances.

F.-J.-H. LACAZE-DUTHIERS. 

En 1853, j'ai parcouru le littoral méditerranéen entre Marseille et Cette, j'ai visité les environs de Barcelome, el exploré les principaux ports des îles Baléares.

Dans ce voyage j'ai pu recueillir de nombreuses observations d'anatomie et de physiologie zoologiques : je les ai publiées pour la plupart isolément.

Aujourd'hui je les réunis.

Ces observations toutes spéciales auraient dù être précédées de recherches sur la faune des localités explorées, surtout des iles Baléares.

Beaucoup font aisément ces sortes de travaux. Rien ne me parait rendre plus incertaine la géographie zoologique que ces énumérations faites à la hâte et presqu'en courant; aussi mes notes ne. un'ayant pas paru assez complètes, je me suis abstenu.

Pent-être ın jour, cédant an désir de revoir le hraul paiys des. 
Balćares, pourrai-je compléter mes observations, at entreprendre ce que je n'ose faire aujourd'bui.

Ce clésir est d'autiunt plus vil que c'est en parcourant ces îles ‘̀ pied, une boitte ì herborisation en santoir, recueillant ici un inseete, là une plante, adınirant partout la beanté dess sites, du ciel et de la mer, allant le soir me reposer dams un couvent où l'hospitalité n’est jamais refusée, yue j’ai ressenti les plus vives impressions, les jouissances les plus vrates, celles tue lait éprouver à l'homme la contemplation de la naturo!

Lorsque ahandonmé à cette riverie ou l'homme aime tant à se: laisser aller pour vive de souvenirs, less diflérents épisodes dr: mon voyage se représentent à ma mémoire, jo me promets de revoir les montagnes de Selva, de Cilimari, de Binisalem, toutes couvertes de Cistes roses ou blanes, ef de Pins dont la résine odorante en s'écoulant des trones sous les ardeurs du soleil embaumait les airs; je me promets de les revoir pour jonir eneore une fois du magnifique speetacle qui s'est offert à mes regards, quand jai apercu du hant de leur sommet, dans un immense horizon, tonte l’île Majorque entourée par les eaux blenes de lat Méditerranée où se détachaient, comme de vagnes et lógères vapeurs blanches, Cabrera, Iviça et Formentera, devenus tontes célèbres par les soulfrances des prisomniers et les travaux des phissiciens framęais; je me rappelle les gorges de Blue, dont les magnifiłues Chênesverts encadraient l'une manière si piltoresque le monastère qui seul, à l'époque où je le visitais, avait pu survivre encore aux révolutions, et où arrivaient le soir des pélerins, souvent des pêchenrs, pour prier me houne Vierge en renom dans les îles, Nostra Señora de Lluc; je n'oublie pas Valdemusa, avee sa belle Chartrense inarlıevée, ses ròtes essarpées et ses vieux Oliviers, dont les 
Iromes monstruens et difformet; do'ssitent entre la mer et la montagne lems silhoueltes bizarres el fantastipues.

Je revois ces magnifiques vallées de Sollers et de Deyha, dont les Oranges délicieuses approvisionnent nos marchés du Nidi. Leur souvenir ne peut s'eflicer de ma mémoire. Quand je les traversai, c’était la Saint-Jean, fëte de la Jemnesse en ce pays. Au milieu de leurs bois d'Orangers que des sources d'une eau vive arrosent incessamment, et font croitre sons ce ciel brulant avec une magiy ue rapidité, ce n’était durant des nuits, merveilleuses de calme el ile sérénité, que chauts et sérémades!

Que de choses à voir dans ces îles, dans ces petits coins de terre jetés au milieu de la ner! Laissant de côté les nombreux somvenirs historiques ef les monmments somptuemx de Palma (fui les rapjellent, le naturaliste et le tomriste devaient entreprendre le voyage de Majorque, n'eussent-ils à y voir, dans le voisinage de la petite ville d'Arta, que les grottes de l'Ermite. Lil, dans le llane d'une montagne s'ourre an-dessus de la mer une immense carerne, qui semble avoir été formée par le ploiement du sol, dans me rivolution du globe. Quand on a gravi, non sans quelque émotion peutêtre, le sentier étroit, tortueux et rapide creusé entre le roc ef les précipices où se brisent les flots ; puand on a franchi l'entrée de la grotte qui sert de refuge, contre les ardeurs du soleil, aux rares troupeaux de chèves de la montagne, et où viemnent nicher des vols de colombes sanvages, on est largement dédommagé de ses fatignes; on pénètre bientôt dans un dédale de colonnes aux mille formes, que la nature semble s’ètre plu à varier de toute manière, el sur lesquelles elle étale, aux yeux étomnés, toules les splendeurs des cristallisations les plus gigantesques. Là, dans les profondeurs de la terre, au milieudes ténèbres épaisses que la lımière rougeâtre et vacillante rles torrhes des guides vient seule écarter un moment; 
dans ce silence profond et absolu qui lait que l'on écoute, saisi que l'on est par cet éloignement romplet de tout mouvement, de tout ètre, de tout signe de vie; qui nese trouverait en face de la nature, ce grand et patient architecte; qui ne se demanderait alors que sont, pour arriver promptement au but, nos fougueux emportements auprès de cette goutte d'eau lentement distillée à la voûte de ces sombres demeures, et dont la chute régulière, constante et monotone élève une colonne aux proportious colossales!

Entreprendrai-je un nouveau voyage aux Baléares, qui me permette d'en faire l'histoire zoologique?

Comment répondre à cette question? L'affirmative est cependant mon désir le plus vif. Alors peut-être pourrai-je faire connaître Albufera et Albuferetta, si riches, dit-on, en Oiseaux de rivages, et dont les marais ne sont abordables qu'au printemps, car les miasmes qui s'en dégagent pendant l'été désolent les contrées voisines d'Alcudia oì le séjour est difficile et bien pénible.

Ferai-je une description détaillée de la faune maritime de Minorque? Elle doit être bien remarquable si l'on en juge par celle de l'admirable port de Mahon qui nourrit tant d'animaux divers; c'est à peine si j'ose l'espérer.

En tout cas, ces localités offrent de riches moissons à celui qui ira les visiter; s'il ne m'est pas permis de les revoir, je serais heureux que ces quelques lignes pussent déterminer d'autres plus favorisés à aller laire ce que je n'ai pu achever dans un premier voyage. 


\title{
M É M O I R E
}

SER

\section{L'ORGANISATION DE L'ANOMIE}

\author{
(ANOMIA EPHIPPIUM).
}

I.

Les études d'anatomie comparée ont toutes pour but, d'une manière plus ou moins éloignée, plus ou moins directe, de rapprocher les organismes afin d'en reconnaître les différences, et de chercher quelles lois ou principes président à leur formation. La nature a multiplié les formes, et eaché, dans bien des cas, sous des détails infinis, des choses différentes en apparence, quoique semblables au fond. Des modifications souvent très sensibles, mais de peu d'importance, conduisent aux innombrables formes que l'anatomiste voit devant lui. Parmi les eauses qui entrainent le plus fréquemment ces transformations, on peut placer les conditions biologiques; que nous en saisissions or non le but, elles jouent un grand rôle dans la modifieation des types.

Ainsi, qu'un poisson soit toujours oceupé à chercher sa proie, en nageant à plat, tout près du fond des lieux qu'il habite, et bientôt 
on le voit revêtir une forme particulière. II devient Pleuronecte, pour employer l'expression consacrée : ses yeux se placent d'un même côté de la tête; sa bouche, ses ouïes se modifient; en un mot, sa forme devient différente de celle du poisson qui nage dans les conditions ordinaires. Par des études comparatives, les ichthyologistes n'ont pas tardé à reconnaitre la cause de ces changements, à donner l'explication de toutes les anomalies, et à pouvoir rapporter l'organisation des Soles ou des autres Pleuronectes au type régulier.

Cet exemple n'a pas été choisi sans raison, comme on le verra; il fera comprendre le but de mes recherches sur l'organisation de l'Anomie. J'ai voulu, en effet, reconnaître si les irrégularités, les anomalies qu'on observe dans ce Mollusque, et que son nom rappelle (1), avaient pour origine une condition biologique particulière, ou bien si elles étaient la conséquence d'un plan tout spécial ; enfin si l'on pouvait, s'expliquant la déformation de l'être, en rap porter toute l'organisation à celle des Lamellibranches les plus réguliers, dont le groupe est si bien caractérisé.

On sait rue l'Anomie vit attachée aux rochers, aux autres corps solides sous-marins, aux coquilles vivantes, et que son adhérence est extrême. Son test prend la forme, l'empreinte des corps auxquels il est invariablement fixé en se moulant sur eux. On sait aussi que c'est à l'aide d'un os très dur, cui passe au travers de l'orifice de l'une des valves, qu'a lien l'adhérence. Cet os a reçu indifféremment le nom d'ossicule ou d'opercule. Sa nature a été diversement interprétée.

Bruguières (2) dit que les Anomies « appartiennent à l'ordre „ des Coquilles multivalves; elles sont formées de trois valves "distinctes... Elles s'altachent sur les corps au moyen de leur " troisième valve, qui, par sa nature, a beaucoup d'analogie avec „les opercules des coquilles univalves. »

Cette opinion ne semble pas ĉtre celle de Poli (3). L'autenr ita-

(1) De arousos, dissimilis.

(2) Articles Avonie, Vers (Mollusques), t. I, p. 70, Encyclopédic méthodique (1789).

(3) Poli, Testucearum utriusque Sicilice, p. 183, $2^{\circ}$ partie. 
lien a décrit avec soin l'ossicule des Anomies, mais il n'en a pas reconnu l'origine.

Cuvier (1) montre le muscle central de l'animal traversant l'ouverture de la coquille, et venant « s'insérer à une troisième pièce ou plaque, tantôt pierreuse, tantôt cornée, par laquelle l'animal s'attache aux corps, » tandis que le reste de ce muscle sert à joindre une valve à l'autre.

De Lamarck (2) pense que l'opercule osseux a été pris à tort pour une troisième valve; il n'est que l'extrémité dilatée et densifiée du tendon du muscle intérieur de l'animal, enfermé de manière à remplir le trou ou l'échancrure du crochet de la valve aplatic.

Dans l'article Anouse du Dictionnaire d'histoire naturelle, M. Deshayes (3) remarque que l'on n'a pas assez étudié l'analogie qui existe entre le Mollusque qui nous occupe et les Térébratules; que « la petite valve des Anomies représente la valve perforée des „ Térébratules; et que l'osselet qui passe ì travers représente le " ligament suspenseur de ceux des Brachiopodes qui en ont un.". Voilà une opinion différente de celles qui viennent d'être eitées, et qui conduit à éloigner des Acéphales lamellibranches le Mollusque qui va nous occuper. II. Deshayes dit que l'on doit le considérer comme intermédiaire entre les Ostréacées et les Brachiopodes (4).

Siebold, dans son Anatomie comparée, consacre un paragraphe spécial aux Lamellibranches privés de locomotion, et qui sont dépourvus de byssus. Il décrit encore l'ossicule comme la terminaison du muscle des valves (5). Ainsi, pour cet auteur, l'Anomie offre une organisation toute spéciale.

MM. Forbes et Hanley, dans un ouvrage récemment publié en Angleterre, ont indiqué à l'état de doute l'opinion qui fait la base de notre travail. Ces auteurs observent que l'Anomie se rapproche

(1) Cuvier, Règne animal, édition illustrée, t. VIII, p. 193.

(2) Lamarck, Animaux sans vertèbres, $2^{\circ}$ édition, t. VII, p. 272.

(3) Dictionnaire dhistoire naturelle de Charles dOrbigny, article Axomf, 1841 .

(4) Loc. cil., p. 559, vol. A.

(5) Siebold, Anat. comp., t. I, P. 2 $9, \S 180$ 
beaueoup du genre Pecten, et que le genre Hemipecten de Reeve est venu combler la lacune qui les séparait. Ils pensent que le sinus auriculaire de la valve plate des Peignes est l'analogue de l'échancrure profonde qu'on remarque sur la valve inférieure de I'Anomie, et ils ajoutent que si l'on avait étudié de très jeunes individus, on pourrait rencontrer l'animal produisant un byssus, qui, passant par le sinus, se fixerait à l'extérieur, et formerait le processus opereulaire avec une partie du muscle addueteur (1).

Ces auteurs citent des extraits d'un travail de M. Clarck, dans lesquels on ne voit point développer cette idée de l'origine de l'opercule; du reste, ì la tin de la citation, on trouve que la cause de l'irrégularité plus ou moins grande est la facilité avec laquelle ces animaux se moulent sur les corps étrangers, dont ils cmpruntent, pour ainsi dire, la forme.

Je n'ai pu trouver d'autres travaux sur l'animal intéressant dont je vais essayer d'esquisser l'anatomie et d'expliquer les formes anomales. Dans ceux que je viens de citer, on peut remarquer des oppositions assez grandes; car admeltre que l'Anomie est tantôt multivalve, tantôt voisine des Brachiopodes, tantôt, au contraire, très rapprochée des Pecten, ou bien enfin croire que le muscle des valves est, pour ainsi dire, fixé par une de ses parties aux corps environnants, c'est montrer quel vague existe encore sur ce Mollusque. Ces raisons m'ont encouragé ì faire connaître les résultats de mes études anatomiques. Je me propose de les faire suivre de recherches d'embrỵogénie, espérant ainsi confirmer les unes par les autres.

(1) Forbes and Hanley, 1850, t. II, p. 323: « ... and when the every young n fry of this genus shall have been carefully observed, we believe they will " found spinning a byssus which, passing through this sinus, fixes the shell in " the first instance, before a portion of it becoming attached, eventually be" comes detached with a part of the adductor muscle, and forms the opercular "process. " 
II.

oRganisation de L'anomia ePHipPIUM (1).

L'Anomie présente, dans tous les points de son organisation, une telle irrégularité, un tel déplacement des parties, que l'on éprouve, en commençant son étude, une peine assez grande à bien s'orienter, et à reconnaître ou fixer la position de quelques organes.

Ce qui frappe tout d'abord, e'est la présence de cet opercule osseux placé à peu près au centre du manteau, qu'il parait avoir perforé. L'animal ressemble, qu'on me passe la comparaison, à un Champignon dont le chapeau serait formé par le manteau et le cöps, et le pédoncule par le petit osselet.

Le manteau, taillé sur le modèle de la coquille, en est, si l'on peut dire, la reproduction; il ne s'agira ici que de lui, l'étude de la coquille trouvant mieux sa place au moment où il sera question du développement : d'ailleurs elle a été parfaitement déerite par les conchyliologistes.

Le côté droit (2) du manteau,-régulier dans la pius grande partic de son étendue, n'est pas perforé en avant par l'opercule, comme on le croirait; il présente une échanerure profonde (3) qui loge celui-ci : l'angle de réunion du bord libre se prolonge en une lan . guette pointue qui entoure l'ossieule (4) à sa base, semble fermer l'échancrure en, avant, et lui donne l'aspect d'un trou. Il n'y a done iei que le prolongement d'un appendice et que son reeroquevillement.

Le voile gauche ne présente aucune partieularité analogue (5).

(1) Anumia ephippium, Bruguières, Encycl. méth. - Lamarck, Anim. sans vert. - Poli, Test. utr. Sicilice.

(2) Dans tout ce travail, la position des organes sera indiquée d'après la position de l'animal, la bouche étant en avant, l'anus en arrière, la charnière en haut, l'ouverture ou manteau en bas.

(3) Pl. I, fig. 2.

(i) Pl. I, fig. $2\left(x^{\prime}\right)$.

(5) Pl, I, fig. 1. 
La réunion sur le dos des deux moitiés du manteau ne se fuit que dans une très courte étendue; elle n'a guère que $\mathbf{1}$ centimètre dans les plus grands individus. On y remarque un orifice (1) dont on ne. peut avoir la connaissance que par les soins les plus ninutieux. Il donne passage au ligament élastique, et à la dent cardinale de la valve plate, droite; et pour peu que l'on ouvre l'animal sans précautions, on déchire la mince languette qui le limite en haut. Il paraît avoir pour cause la soudure de deux prolongements qui entourent ce ligament, et passent derrière lui. Ce doit être quelque chose d'analogue à ce qui s'observe dans les Myes, et surlout dans les Pholades.

L'animal est presque rond et très aplati. On distingue sur les deux faces des muscles, bien différents en nombre, suivant que l'on regarde à droite ou à gauche; ils occupent à peu près le centre de la figure. A droite, on n'en voit qu'un (2), presque circulaire ; il est placé en arrière de l'ossicule (3). 1 gauche (4), on en compte trois, formant une série courbe, à concavité antéro-inférieure. Chacune des masses est forte et bien développée. Enfin, en avant, et tout à fait sur le bord du manteau, près de la petite perforation dorsale dont ill vient d'être question, on distingue un quatrième muscle $(5)$ peu considérable, qui ne paraît pas avoir élé indiqué. Cette différence dans le nombre des paquets musculaires a évidemment embarrassé les auteurs qui n’ont pas fait de longues recherches anatomiques; aussi voyons-nous que le muscle adducteur des valves a été considéré comme très volumineux, ct se partageant en deux parties, mies du côté gauche, séparées à droite, et se rendant l'une à l'ossicule, l'autre à la valve.

Cette opinion n'est nullement justifiée par les dissections; en ellet, le muscle, qui semble au centre du lobe droit (6), est le

(1) Pl. I, fig. 2 (z).

(2) Pl. I, fig. $2(y)$.

(3) Pl. I, fig. $2\left(x^{\prime}\right)$.

(4) Pl. 1, fig. $1(x, x, y)$.

(5) P. I, fig. $1(v)$.

(6) Pl. I, fig. $2(y)$. 
mème que celui qui parait le plus en arrière du côté gauche (1). Sans beaucoup de difficulté et d'attention, on peut le détacher nettement, et l'on voit ses fibres réunies formant un cylindre ne pas se mèler ì celles des autres faisceaux.

Ceux-ci '2) se dirigent vers l'opercule, et s'insèrent sur son extrémité supérieure, très inégale, couverte d'aspérités. Poli (3) a très bien vu les différents faisceaux; il les a déerits avee grand soin, et a donné sur eux beaucoup de détails ; mais il paraît tomber dans l'opinion commune : car il croit qu'on doit les considérer comme produisant un seul muscle, ru'il nomme muscle à quatre têtes "Musculus hic quadricipitis denominationem recte meretur." La distinetion s'établira nécessairement, quand l'organisation de l'Anomie sera rapportée à celle des autres Acéphales; et elle sera mieux comprise qu'elle ne le serait maintenant. Pour le moment, on peut établir que les deux masses antérieures, qui se voient sur la face du lobe gauche du manteau, sont les museles rétraeteurs de l'ossieule, tout à fait différents du muscle des valves, et que l'une est un peu antérieure à l'autre.

Quant au petit faiseeau (4), qui a été remarqué tout près de la marge antérieure du manteau, il appartient au pied.

Le manteau de l'Anomie, loin d'être séparé du corps de l'animal comme dans les autres Mollusques, lui adhère par sa face interne du côté droit; aussi sa description ne peut-elle être complétée ici, elle le sera quand nous étudierons les organes génitaux et le tube digestif.

Organe de la digestion. - Il y a dans l'ensemble de l'appareil qui sert à l'alimentation une anomalie bien grande, qui, au premier abord, est très embarrassante ; on éprouve même de la difficulté à en suivre les dispositions.

La bouche(5) est placée du côté droit, entre deux voiles labiaux, un peu en avant et en haut de l'opercule; celui-ci, quand il est en-

(1) PI. I f fig. 1 (y).

(2) Pl. I, fig. $1\{x, x\}$.

(3) Testac. utr. Sicilice, $2^{\mathrm{e}}$ partic, $\uparrow .181$ i 185

(4) Pl. I, fig. $1(v)$.

(5) Pl. 1, fig. 4 (b), fig. $6(b)$. 
core en place, paraît entouré par un cercle complet, que forment, en arrière et en bas, les branchies, en avant et en haut les lèvres. Dans ce cercle est compris aussi un petit appendice (1) musculaire, qui rappelle par sa forme le pied de la Moule ou des Peignes : c'est le pied que l'on trouve, en général, caché sous l'ossicule.

Les lèvres sont des replis à peine saillants, très rapprochés, se terminant aux extrémités antérieures des branchies, et se dilatant un peu au niveau de la bouche. Leurs extrémités voisines des organes de la respiration sont disposées de telle sorte, que la lèvre postérieure semble se continuer avec les lames internes, tandis que la lèvre antérieure s'unit aux lames externes $(2)$. Comme la branchie gauche est beaucoup plus longue que la droite, un rapport inverse existe dans les proportions des moitiés gauches et droites des lèvres; c'est ce qui les fait paraitre presque entièrement sur le côté droit de l'animal. Remarquons que si, dans les Mollusques acéphales, les parties latérales des voiles labiaux sont celles qui se développent le plus, ici, au contraire, c'est la partie moyenne : la bouche, infundibuliforme, assez grande, et regardant à droite, est cachée par la dilatation des lèvres. Sa position la met en rapport avec le bord contourné du manteau (3), qui entoure l'osselet en se roulant en spirale.

L'osophage est tellement court, que c'est à peine s'il mérite ce nom.

L'estomac fait, pour ainsi dire, suite immédiatement à la bouche; il est creusé dans l'intéricur de la masse du foie. Par une coupe (4) de la partie du corps qui reste entre les deux lobes du manteau, on met à découvert une multitude de cavités irrégulières, inégales, rappelant grossièrement la disposition des glandes en grappe, creusées dans la substance hépatique, et y formant autant de loges où tombe la bile et où viennent se placer les substances alimentaires. Du côté du dos, ces loges sont plus nombreuses. La cavité générale de l'estomac, résultant de tous ces enfoncements,
(1) Pl. I et pl. II $(p, p)$.
(2) Pl. I, fig. 4.
(3) Pl. I, fig. 2.
(i) Pl. I, fig. $3(i)$. 
le toutes ces anfractuosités, n'a donc pas de forme appréciable ; elle est tapissée par une muqueuse, dont l'épithélium vibratile est remarquable de vivacité. Sur aucun autre exemple, je n'ai vu, avec autant de netteté et d'évidence, l'apparence d'une série de perles roulant avec une régularité extrême.

En arrière, vers le muscle des valves, la cảvité communique avec deux orifices, ou plutôt deux orifices distincts s'abouchent à côté l'un de l'autre dans une même anfractuosité : l'un est supérieur, l'autre est inférieur. Le premier conduit dans l'intestin, le second dans le coecum.

L'intestin (1), très court, presque droit, marche directement d'avant en arrière, et se courbe un peu en passant au-dessus du muscle des valves, puis se termine à l'anus en formant un tube libre assez long. Dans son trajet, il ne décrit aucune circonvolution; il traverse la partie postérieure de la masse abdominale, formée en arrière du foie par les organes génitaux. Sa largeur égale tout au plus 1 centimètre; il mérite bien le nom de rectum, d'après ses caractères, mais il représente tout l'intestin. Nous verrons, en parlant du cœur, qu'il passe sous lui, et non dans son intérieur.

Le cœcum (2) occupe une position, et acquiert un développement bien remarquable. Les auteurs semblent ne pas s'en être occupés. Il nait immédiatement au-dessous de l'intestin, se porte d'abord en arrière, se dirige ensuite en bas et à droite en décrivant une courbe à concavité antéro-supérieure, et gagne le manteau, dans le lobe droit duquel il se loge complétement. Dans ce trajet (3), il passe entre l'os et le muscle des valves, croise la branchie droite, et marche un moment à côté de l'organe de Bojanus. Dans le manteau (4), il est en rapport avec les organes de la génération, au milieu desquels il est placé ; on le voit remonter très haut en avant, et salongueur étonne quand on la compare à celle de l'intestin. Il semble qu'il y a compensation de la brièveté de l'un par l'allongement de l'autre. Dans son intérieur, on trouve un stylet hyalin,

(1) Pl. I, fig. $3(l)$.

(2) Pl. I, fig. $3(q)$.

(3) Pl. I, fig. $3(q)$; fig. $5(q)$.

(4) $\mathrm{Pl}$ I, fig. $3(s)$. 
analogue à celui que l'on rencontre chez la plupart des Acéphales lamellibranches qui le remplit complétement, et dont la nature, ainsi que les fonctions, est encore inconnue.

Le foie (1) représente à peu près à lui seul toute la masse abdominale; il entoure de toutes parts l'estomac; sa teinte et sa consistance, de même que sa structure, rappellent beaucoup ce que l'on observe dans l'Huitre : de telle sorte que, sur une coupe, il paraît compacte et dense. A gauche et en arrière, il est recouvert par une petite portion des glandes sexuelles; celles-ci, d'une leinte jaunâtre ou rouge assez vif, s'en distinguent facilement, ì eause du fond brun foncé qu’il forme.

La texture de la glande hépatique ressemble beaucoup à celle qu'on observe dans les autres Acéphales. On y remarque des acini en forme de eul-de-sac, remplis par de la matière jaune caractéristique; celle-ci est formée de cellules gorgées de granulations. L'intérieur de ehaeun des acini est creux et rempli de liquide, dans lequel flottent les cellules du parenchyme sécréteur. Les parois sont tapissées par un épithélium vibratile des plus évidents, qui se continue avec celui de la muqueuse stomacale.

Organes de la respiration. - Les branchies s'observent entre les deux voiles du manteau avec des courbures et des formes diverses, suivant qu'on les examine à droite ou à gauche. La branchie droite (2) est plus courte que la gauche; elle est aussi beaucoup plus courbée, et décrit presque les trois quarts d'une eirconférence autour du musele postérieur des valves, dont elle est séparée en avant par l'insertion du manteau. Son extrémité antéricure est éloignée de la bouche par toute la longueur de l'ossicule, et son extrémité postérieure est libre, recourbée vers l'anus. Celle du côté gauche(3) monte beaucoup plushaut, et vient se terminer en avant de l'opereule, non loin de la bouche; son extrémité postérieure, égale à celle de la branchie droite, est libre; aussi peut-on dire qu'elle est plus longue et moins courbée.

(1) Voyez dans les différentes figures $(f)$.

(2) Pl. I, fig. 5 ; pl. II, fig. 1.

(3) Pl. 1, fig. 5 ; pl. 11, fig. 2. 
Les feuillets (1) sont au nombre de quatre de chaque côté : deux pour chaque branchie, un moyen et un réfléchi. La lame interne, réfléchie du côté droit, se soude, par son bord libre ou supérieur, avec celle du côté opposé. La soudure a lieu dans toute la longueur; aussi, pour arriver à voir les organes profonds, faut-il détruire les adhérences.

En général, dans les Lamellibranches, quand les deux feuillets réfléchis des deux branchies internes s'unissent sur la ligne médiane, ce n'est jamais dans toute leur étendue ; presque toujours, les moitiés antérieures se trouvent séparées par l'abdomen de l'animal. Parmi les exemples fort nombreux, je ne citerai que les Unio, Anodonta, Cardium, Solen, etc., ete. Dans l'Anomie, il n'en est pas de même ; la jonction s'effectue sur toute la longueur du bord des lames internes, et cela avec une particularité qui peut embarrasser au premier abord. Le pli interne du côté droit se porte un peu à gauche, de telle sorte qu'il semblerait, en n'y regardant pas de très près, que la branchie droite n'a que trois feuillets, tandis que la gauche en aurait cinq ; il suffit d'être averti pour ne pas faire d'erreur.

On observe en dehors de la lame externe ou réfléchie de la branchie externe une lamelle très petite, qui forme réellement une cinquième lame (2). Cette circonstance n'est pas commune parmi les Acéphales; elle mérite donc d'être signalée. Le bord inférieur en est libre, et ne descend pas jusqu'au bord inférieur des autres feuillets; il ne porte pas de lame réfléchie, et reste simple.

Les branchies, en arrière du corps et de la masse abdominale, sont portées par des replis falciformes (3), dont le tissu brunâtre ressemble par sa structure à un corps spongieux. Le manteau (4) adhère en quelques points avec cette partie, base d'insertion de l'organe de la respiration, et dont nous étudierons la texture en nous occupant de la circulation.

La structure des branchies de l'Anomic ressemble, à certains

(1) Pl. I, fig. 4.

(2) Pl. I, fig. $4(h)$.

(3) Pl. II, fig. 1 et 2.

(4) Pl. I, fig. $4(u)$; pl. II, fig. $1(u)$. 
égards, à celle que l'on observe dans la Moule, et surtout le Pcigne (1). Les filaments, les rayons qui en forment les lames, sont à peine unis entre eux, et il n'est guère possible d'y toucher sans les diviser en une foule de débris qui flottent dans l'eau, comme une multitude de dents de peigne.

Organes de la circulation. - Nous n'étudierons ici que la disposition des centres et des ramifications principales; car c'est surtout sur ces parties que portent les anomalies. La circulation des Mollusques a été l'objet de recherches si importantes, que l'utilité des . détails serait au moins contestable ici. Je renverrai done aux magnifiques travaux de $\mathbf{1 1}$. le professeur Edwards (2).

Le cœur (3) présente des anomalies bien curieuses et bien rares, si même elles cxistent dans d'autres Mollusques ou dans le règne animal. Il est placé, comme toujours, en arrière de la masse hépatique, au-dessus du musele des valves et un peu en avant de lui; mais tandis que, dans les autres Acéphales, il est enfermé dans un péricarde, clos de toutes parts sans communications directes avee l'extérieur, ici, au contraire, il est libre, et paraît comme une petite sphère, dans la dépression que l'on remarque tout près du rectum, en avant de l'extrémité postérieure des branchies. C'est là un fait singulier dans l'histoire des organismes, que de voir la partie centrale de l'appareil de la circulation placée en dehors du corps, auquel il semble suspendu par un court pédoncule. Poli (4) avait reconnu cette disposition; elle cxcita même son admiration : "Nunquam me hercule mirari desinimus, "s'écrie-t-il après avoir fait contracter le ventricule par de légers attouchements, et il ajoute : "Cor adeo nuda atque detecta reliquisse ut et iteratis arence ictibus " et jugi aquarum collisioni obnoxia sint. "

Est-ce à dire que le péricarde n'existe pas? Ce serait pent-être s'avancer un peu trop que de l'affirmer. Il est probable que le cœur était recouvert par une enveloppe qui s'est moulée sur lui, et qui

(1) Mytilus edulis, Pecten Jacobaus.

(2) Recherches sur la circulation des Mollusques. - Voyage en Sicile, $18 \$ 3$.

(3) Pl. II, fig. 1 et $2(V)$.

(i) Testacearum utriusque Sicilice, $2^{\mathrm{e}}$ partie, p. 184. 
s'est soudée arec ses parois; que l'un et l'autre sont protégés par la couche générale épithéliale du corps ; mais la fusion des trois parties est telle, que quelque soin que l'on apporte à les séparer, on ne peut y réussir. Jamais je n'ai pu parvenir à voir une cavité quelconque qui pût déceler la présence du péricarde.

Le ventriculc a un volume variable; on le rencontre sur des individus les plus grands avec un diamètre de 5 millimètres; mais le plus souvent les proportions sont de beaucoup inférieures, et, sans aucun doute, elles doivent changer avec les mouvements de systole et de dyastole.

Quant à l'observation de ces contractions, j'ai été bien moins heureux que Poli (1); je n'ai jamais pu les apercevoir. On sait avec quelle facilité, dans la plupart des Acéphales, on peut les observer, soit qu'elles se produisent naturellement, soit qu'elles naissent par des excitations. Malgré toute mon attention, je n'ai jamais pu les faire naitre. Les animaux que j'observais élaient parfaitement vivants; le cœur n'avait pas été blessé. On comprendrait, d'après les explications de Poli, que le ventricule doit être, en effet, peu sensible aux attouchements, puisqu'il est en contact incessant avec l'eau, le sable et les corps étrangers. Peut-être les animaux que j'ai eus entre les mains n'étaient-ils pas dans des conditions convenables ; toujours est-il qu'à Mahon, pas plus qu'à Cette (2), je n'ai pu voir de contractions analogues à celles que l'on voit si facilement dans tous les Acéphales; je suis loin d'en conclure qu'elles n'existent pas, mais je crois qu'elles se font d'une manière plus insensible et plus lente.

Une autre particularité se présente non moins importante que les précédentes : on sait que, dans les Acéphales lamellibranches, le cœur est traversé par le rectum; cela s'observe dans une foule

(1) Poli, passage cité précédemment.

(2) Depuis la rédaction de ce mémoire, j'ai dans d'autres localités, à Granville, à Saint-Malo, à Saint-Jacut-la-Mer en Bretagne; observé très distinctement ces contractions; elles sont lentes, mais elles peuvent être déterminées par les attouchements. Je dois avouer ne pas comprendre comment, sur les Anomies de la Méditerranée, je n’ai pu voir une chose qui seproduit si nettement sur a même espèce de la Manche et de l'Océan. 
d'espèees, comme l'a démontré M. le professeur Edwards dans ses belles recherches sur la circulation, et comme l'avaient vu Poli et autres. L'Anomie fait exception à cette règle; l'intestin passe au-dessous du cœur, qui forme avec les oreilleltes comme un pont véritable au-dessus de lui. Cette différence exceptionnelle doit certainement se ratlacher à la première anomalie, qui a détaché pour ainsi dire le cour de tous les organes. Ici nos observations ne sont pas d'accord avec celles de Poli, qui admet le passage du reclum au travers de l'organe central de la circulation : "Rectum..... " ex abdomine emersum cordis cavitatem pertransire visum est. "

Les oreillettes (1), placées latéralement de chaque côté du cœur, varient beaucoup de position, de direction, de forme et de volume, à droite et à gauche.

L'oreillette droite (2) est, comme le ventricule, placée extérieurement; on la voit dès que l'on a enlevé le manteau; elle devient très évidente sur la face à droite. Si l'on pousse un liquide coloré dans le cordon cylindrique qui, du ventricule, se rend aux branchies en décrivant une courbe concentrique à leur bord d'insertion, les injections dilatent l'oreillette, et la mettent bien en évidence. Quand on injecte le ventricule, les liquides ne pénètrent pas dans celle-ci, ce qui indique la présence de valvules auriculo-ventriculaireś, semblables à celles qu'on ohserve dans les autres Mollusques.

L'oreillette gauche est moins distincte; on ne l'aperçoit que lorsqu'on a écarté les parties quil l'entourent. Il est nécessaire de la gonfler par une injection; elle (3) est placéc dans l'enfoncement qui existe entre les deux masses museulaires de l'ossicule en avant et le muscle des valves en arrière; elle a la forme d'un ovoïde allongé, dont le grand diamètre est dirigé d'avant en arrière, c'est-à-dire dans un sens absolument opposé à celui de l'oreillette droite qui se dirige de haut en bas. Ces différences sont en rapport, on le comprend, avee les variations de formes des branchies et des autres parties du corps.

(1) Pl. II, fig. 1 et $2(O D),(O G)$.

(2) Pl. II, fig. 1 (OD).

(3) PI. II, fig. 2 (OG). 
Circulation branchiale. - En poussant une injection par l'oreillette droite, et l'on réussit presque toujours facilement, on voit le liquide coloré suivre un vaisseau (1) circulaire, concentrique an bord adhérent des branchies, et arriver jusqu'ì leur extrémité postérieure. Il n'est pas douteux que l'on n'ait rempli le conduit qui rapporte le sang de l'organe de la respiration au cœur ; cela est évident, par cette raison que le lirguide pénètre de ce vaisseau dans le ventricule, mais que du ventricule il est impossible de le faire aller vers les branchies : ce sont les vaisseaux branchio-cardiaques.

Les différences de forme des deux organes de la respiration entraînent après elles des différences correspondantes dans la marche des organes de la circulation. Ainsi, à droite (2), après avoir quitté l'oreillette, le vaisseau branchio-cardiaque marche d'abord parallèlement au repli du manteau, aux voiles labiaux, et se dirige de haut en bas vers le bord concave des branchies, qu'il suit dans toute son étendue. A gauche, au contraire (3), il se porte directement en avant entre la masse hépatique et les muscles de l'ossicule ; il contourne ceux-ci en se portant d'abord directement en avant pour atteindre l'extrémité antérieure de la branchie gauche, que nous avons vue s'avancer beaucoup en avant. Il suit alors le bord concave de cette portion de l'organe, et arrive jusqu'à son extrémité postérieure.

Ces deux canaux, en suivant la ligne courbe d'insertion des branchies, reçoivent, perpendiculairement à leur direction, les vaisseaux capillaires qui viennent des rayons.

Le sang qui va aux branchies pour respirer et se transformer est distribué dans leur intérieur par un ensemble spécial de canaux, dont la réunion forme un véritable corps spongieux. 11 arrive des différentes parties du corps par un vaisseau unique (4). placé à la base de chacun des replis falciformes portant les branchies. En écartant celles-ci, on le voit près du muscle adducteur des valves; il ne tarde pas à se diviser en deux branches secondaires, l'une anté-
(1) Pl. II, fig. 1 (BC).
(2) Pl. II, fig. 1.
(3) Pl. II, fig. 2.
(4) Pl. II, fig. 3 (SV). 
rieure, l'autre postérieure, qui suivent le bord courbe du repli falciforme près de son insertion au corps, et qui sont séparées par conséquent des branchies par un assez grand intervalle. Le sang s'en échappe par une multitude de conduits secondaires capillaires, se répand dans un réseau dont la richesse est telle, qu'une injection poussée avec le moins de soin colore en masse toute la partie. Un canal à parois vagues, véritable sinus (1), parallèle et voisin du vaisseau branchio - cardiaque, recoit le sang pour le faire passer dans les branchies par une foulle de petites dilatations faisant saillie en dehors, et produisant comme une série de petits sinus lamellaires secondaires, servant d'intermédiaire entre lui et les ares branchiaux. Ces petits sinus sont très apparents sur la face interne d'insertion des branchies, et paraissent comme une série de feuillets en nombre à peu près égal à celui des rayons (2), c'est-à-dire en nombre très considérable, et dont le plan est perpendiculaire au plan des lames branchiales : telle est la disposition des vaisseaux des branchies.

Circulation artérielle. - On vient de voir le sang arriver aux branchies, se distribuer dans leur intérieur, et revenir au coeur par les vaisseaux qui le conduisent aux deux oreillettes. Voyons maintenant comment, après avoir respiré, il est de nouveau répandu dans tout le corps. Entre les deux oreillettes, le ventricule est uni en avant, à la partie postérieure de la masse viscérale, par un conduit un peu dilaté, d'où naissent les artères principales. Ce vaisseau qui fait suite au cour, et où prennent leur origine les artères, mérite le nom de bulbe artériel (3). On l'observe facilement, ear il est placé en dehors del'organisation, exactement comme le cour.

Il fournit trois ordres d'artères, dont la direction et la présence sont loin d'être constantes. Habituellement on trouve une branche qui se porte directement en avant en suivant le bord convexe de la masse viscérale, et en fournissant à droite et à gauche des rameaux aux parties qu'elle rencontre : c'est l'artère principale anté-

(1) Pl. II, fig. $4\left(\mathrm{SV}^{\prime}\right)$.

(2) Pl. II, fig. 3, 4.

(3) Pl. II, fig. $5(a)$. 
rieure (1); elle porte done le sang aux parties du côté de la bouche. A gauche de son origine, on voit sur le bulbe aortique naître une artère, que par opposition à la première on peut nommer artère principale postérieure (2); elle passe en dedans de l'oreillette gauche qu'elle eroise, pour arriver dans l'espace qu'on observe entre le. muscle de l'ossicule et celui des valves. Arrivée auprès du dernier, elle se divise en deux branches : l'une, inférieure, s'introduit entre les museles pour aller se diviser daus les parties qui les avoisinent; landis que l'autre, en remontant un peu, rencontre le rectum, qu'elle accompagne d'abord en lui foumissant quelques ramuscules, qu'elle abandonne ensuite pour se distribuer dans le manteau. Le muscle des valves (3) est done enfermé dans un cercle artéricl.

La troisième branche est loin d'être constante : on la trouve en haut de l'artère antérieure principale; elle va se distribuer anx portions postérieures de la masse viscérale.

Une disposition assez fréquente est celle où, du bulbe artériel, naissent deux trones : l'm, inférieur, se porte sur la masse viscérale, tandis que l'autre remonte en se courbant, et se divise à la hauteur de l'oreillette en deux rameaux secondaires qui correspondent, le postérieur à l'artère postérieure principale, l'antérieur à une seconde artère antérieure. Alors la masse viscérale est parcourue par deux artères principales antérieures.

En résumé, la circulation de l'Anomie se passe à peu près comme dans les autres espèces, seulement avec des particularités dont la déformation des organes est cause et rend parfaitement compte.

On sait que dans les Mollusques, en général, il y a une partie du sang qui revient du manteau au cour sans passer par les branchies; en sorte que ces animaux ne sont pas absolument, comme l'a démontré pour beaucoup d'entre eux MI. Edwards, à sang complétement rouge, c'est-ì-dire à sang ayant été tout entier respiré. Le vaisseau (4) qui s'observe, surtout au côté gauche, vers l'extré-

(1) Pl. II, fig. 5 $\left(a^{\prime}\right)$.

(2) Pl. II, fig. $5\left(a^{\prime \prime}\right)$.

(3) Pl. II, fig. $5(y)$.

(4) Pl. II, fig. :5 (e). 
mité postérieure de l'oreillette de ce côté, et présente, le plus souvent, trois branches placées entre le muscle des valves et le muscle de l'ossicule, rapporte au cœur le sang veineux dont il est ici question.

Système nerveux. - Le système nerveux de l'Anomie présente une disposition tout à fait analogue à celle qu'il offre dans la plupart des Acéphales lamellibranches; les différences que nous allons indiquer sont toutes les conséquences des changements de formes déjà si souvent signalés.

La paire de ganglions labiaux, buccaux ou cérébroïdes (1), est placée sur le côté de la bouche dont clle suit le changement de position. L'un des ganglions, le droit, se trouve reporté bien en arrière sur le milieu de la face de l'animal. Il est très facile à distinguer, même sans préparation; on le voit, en écartant les lèvres, en arrière de la bouche, dans le sillon qui lui fait suite. Sa teinte, légèrement orangée, permet de le distinguer très nettement. Il est un peu triangulaire; sa base est tournée vers la bouche, tandis que son sommet regarde en arrière; de chacun de ses angles partent deux cordons qui l'unissent aux autres ganglions.

Quant au ganglion buccal lgauche, il est très difficile à mettre à découvert. On le trouve en dedans des lèvres, à peu près à la hauteur de l'extrémité de la branchie et en dehors du pied. Il se trouve au milieu des paquets musculaires qui se rendent à celui-ci, et comme il est très petit, on éprouve une très grande peine à le trouver: il faut avoir recours à des préparations fort laborieuses.

Les ganglions pédieux sont, à la base même du pied, extrêmement rapprochés l'un de l'autre; ils semblent confondus en une seule masse à peu près sphérique, dont la teinte sert encore à les faire reconnaître. Ils sont moins difficiles à découvrir que le ganglion buecal gauche, dont ils sont très voisins.

La paire branchiale (2), beaucoup plus volumineuse que les précédentes, est ici, comme dans tous les Acéphales, très facile à reconnaître. On la voitsur la face inférieure du muscle des valves;

(1) Pl. I, fig. $6(i, k)$. $-I b$., fig. $5(i)$.

(2) P. I, fig. $5(\mathrm{~g})$. 
elle ne présente pas de particularités très remarquables, à part des irrégularités, conséquences des déformations générales.

Ces différents ganglions sont unis entre eux par des commissures dont la longueur varie; celle des ganglions branchiaux paraît encore, bien qu'elle soit très courte, tandis que dans la paire pédieuse on ne la distingue pas du tout. Celle qui unit les ganglions cérébraux est très longue; elle fait le tour de la bouche en restant logée dans la base de la lèvre supérieure : elle fournit le moyen le plus sûr d'arriver au ganglion gauche que l'on trouve difficilement, si on le clıerche sans se guider sur la commissure qui le joint à son homologue.

Les connectifs méritent d'attirer surtout l'attention. Celui qui joint le ganglion buccal droit au ganglion branchial suit à peu près directement l'insertion des voiles labiaux jusqu'à l'extrémité antérieure des branchies, puis il croise le corcum pour arriver au-dessous du muscle des valves. Il est facile à distinguer dans cette dernière partie de son trajet. Du côté gauche, le connectif, plus gros et plus courbe passe en dehors du pied, et du muscle de l'ossicule en suivant d'abord l'insertion de la branchie, mais en s'en éloignant ensuite pour se porter en dedans.

Les connectifs unissant les ganglions buceaux aux ganglions pédieux présentent une longueur bien différente. Celui de droite passe en avant de l'opercule pour venir dans la base même du pied; quoique très grêle, on peut encore le disséquer et le reconnaître, sa longueur est assez grande; tandis que celui du côté gauche (1), extrêmement court, rapproche beaucoup le ganglion buccal de cc côté du centre nerveux abdominal. Si l'ou joint le développement de l'un à la petitesse de l'autre, et surtout à la taille considérable du connectif, on comprendra comment il y a vraiment une difficulté extrême à bien reconnaittre le ganglion buceal gauche.

En résumé, on peut retrouver les éléments généraux du sỵstème nerveux, et l'on voit la bouche entourée par un cercle de cordons en dehors duquel sont placés le pied et l'ossicule.

Des ganglions branchiaux partent quelques nerfs, dont le déve-

(1) Pl, I, fig. 6 . 
loppement est en rapport avec les organes auxquels ils vont se: distribuer. Tel est, par exemple, celui qui, du côté droit, remonle vers le muscle de l'opereule. Il ressemble, par sa taille et sa direclion, all connectif bueco - branchial; on pourrait d'abord prendre l'un pour l'autre, aussi est-il besoin d'être averti pour ne pas les confondre. Le nerf palléal droit est très développé, et ressemble aux cordons destinés aux branchies; mais en le suivant, le doutr. n'est pas possible. Ces données suffisent pour arriver au but que nous nous proposons d'atteindre dans ce travail. On comprend, à priori, que bien des différences secondaires doivent se présenter, mais que toutes sont la conséquenee des irrégularités plus générales qui précèdent.

Organes génitaux. - L'Anomie est un Acéphale bissexue, ou din̈̈que. Dans un travail général sur le sexe des Acéphales lamellibranches, je crois avoir démontré que l'hermaphrodisme est dans cette classe véritablement peu fréquent, comparativement aıx espèces ayant les deux sexes, et qui forment un groupe que, par opposition an premier, on doit nommer monö̈ques. Il est done inutile de reprendre ici toutes les preuves à l'appui de celte manière de voir; mais, je crois, d'après un grand nombre d'observations qui me sont propres, que l'on doit regarder l'Anomie comme diö̈que, e'est-à-dire à sexe séparé. A vee R. Owen (1), Sicbold (2), il faut done rejeter l'hermaphrodisme que Garner (3) et Poli (4) nous ont montré comme la condition habituelle du sexe des Lamellibranches.

L'ovaire, comme le testicule, occupe pen d'étendue sur la partie postérieure de la masse viscérale; ils s'étendent davantage en avant sur le côté gauche que sur le côté droit; mais, par une disposition très rare dans l'organisation des Acéphales, on observe (5) que toute la fare interne du lobe droit du manteau est converte par

(1) R. Owen, Leclures on the comparalive analomy and physiology, t. I, ए. 287.

(2) Von Siebold, Anat. comp., trad. française, t. I, p. 286.

(3) Garner, Charlesworlli's magazine of natural hislory. 1. II, p. ๕.8.

i) Poli, Teslac utrinsque Sirilice, t. II, Anomia.

j) Pl I, fig. $3(s, s, s)$. 
l'une ou l'autre de ces glandes, qui lorment des bourcelets au milieu desquels on a vu le erecum se loger. Quel que soit le sexe, toujours les bourrelets dont il est iei ruestion sont remplis d'une substance jamàtre qui ressemble à du tissu adipeux; toutefois Poli (1) a vu dans quelques exemples l'ovaire d'une belle couleur rouge. Les masses qui composent la partie palléale de la glande conmuniquent avec celles placées sur le foie en deux points principaux : l'un (2) est dans le voisinage du rectum, l'autre (3) un peu att-dessus de la dépression où est logé le cour. Ce sont deux véritables ponts formés par de la substance glandulaire.

Les oufs se développent dans les cellules qui tapissent les extrémités en cul-de-sae des tubes sécrétenr's réunis en grappe. Its présentent une enveloppe vitelline, mitellus granuleux, une vésienle transparente ou de Purkinje, une ou plusieurs laches germiuatives. Les culs-de-sac séeréteurs, ou se réunissan!, forment des lobules primitifs en général peu distinets; mais enfin la structure et la texture de la glande femelle ne paraissent pas différentes de celles que j’ai indiquées dans un travail général sur les sexes des Lamellibramehes.

Les spermatozoüdes 4 sont du nombre de ceux qui ont une tète globuleuse : leur queue est très déliée. Ils se meuvent avee beaucoup d'agilité, et se développent daus des rellules spéciales qui tapissent la face interne des culs-de-sae sécréteurs. Il n’y a là rien qui ne se présente dans les Acéphales en général; aussi l'ouf et les spermatozoüdes doivent-ils être eonsidérés comme le produit de la sćerétion qui a lieu dans des cellules dont se dépouille le tube sécréteur.

Les eanaux excréleurs dans l'un et l'autre sexe sont tapissé. d'un épithélium vibratile très vif, et viennent s'ouvrir daus la glande de Bojanus.

(1) Testac. utriusque Sicilice, 2ّe part., Anomia. - Sur les Anomies de la même espèce que jai observées depuis dans l'Océanie, à Saint-Jacut, en Bretagne, la couleur rouge s'est présentée très habituellement; elle n'avait rien de vif, el siaprochait un peu du rouge brique.

(2) PI. I, fig. $3\left(u^{\prime}\right)$.

(3) Pl. 1, fig. (u).

i) II. II, fir. 7 
Organes de Bojanus. - Dans presque tous les Lamellibranches, on trouve un sac à parois glandulaires, sur les fonctions duquel on n'est pas d'accord, qui, pour Bojanus, était un poumon, pour Neeuyler un testicule, pour les auteurs modernes un rein, et qu'on peut, à certains égards, regarder comme annexé aux organes de la génération. Quoi qu'il en soit de ces opinions, l'organe de Bojanus (1) est très évident dans les Anomies; on le trouve en dedans del'insertion des branchies, dans la partie antérieure au muscle des valves; aussi, tandis qu'il est allongé à gauche et parallèle à l'axe du corps, il devient courbe et perpendiculaire à cette direction du côté droit. Cette différence nous explique comment la forme de l'un est celle d'un boyau allongé, tandis que celle de l'autre représente un croissant; comment, à droite, le connectif venant du ganglion buccal croise sa direction, quand à gauche, au contraire, il la longe. Enfin, en arrière de l'organe droit, on voit un eylindre transparent décrivant une courbe en sens inverse : c'est le cœecum qui se porte vers le manteau. Des deux côtés, l'organe se termine dars l'angle que forment les nerfs branchiaux et les connectifs branchio-buccaux. C'est dans cet angle qu'il faut chercher son orifice (2); et ici la position se trouve absolument la même que dans les Acéphales en général, où toujours les orifices de la génération et de l'annexe sont placés en dehors des cordons nerveux.

La texture de l'organe de Bojanus est la même que dans tous les Acéphales lamellibranches. Un tissu (3) exclusivement cellulaire, des vésicules lâchement unies, remplies de granulations tantôt brunâtres, tantòt jaunâtres, tantôt d'un joli violet, composent la partie sécrétante; une couche interne de ces cellules couverte de cils vibratiles forme un épithélium dont les mouvements sont très vifs.

(1) Pl. I et II $(r)$.

(2) PI. I, fig. 5 (c).

(3) PI. II, fig. 6. 
III.

CONSIDÉRATIONS GÉNÉRALES.

On vient de voir les anomalies que présentent la position et la forme des divers organes. It reste maintenant à en découvrir la cause, à montrer qu'elles sont la conséquence d'une condition biologique particulière, et que le plan qui a présidé à la formation de l'organisme de l'Anomie est le même que celui sur lequel sont constitués les auitres Lamellibranches.

On est frappé, dès le commencement des recherches, de l'importance et de la prépondérance que prend le côté droit sur le côté gauche. Le manteau, qui se découvre le premier, est épais, et renferme en grande partie la glande génitale et un prolongement du tube digestif ; tout le reste du corps semble tourné de ce côté; le pied, la bouche, les branchies, et surtout l'ossicule, semblent en occuper le milieu. Aussi ne tarde-t-on pas à se convainere qu'il y a eu une sorte de torsion, qui a reporté vers ce côté tous les organes. La cause est certainement, d'une part, la puissance considérable d'un byssus devenu osseux, et de l'autre sa position forcée sur le côté droit. En d'autres termes, l'ossicule de l'A nomie est un byssus, et toutes les anomalies sont la conséquence de la position de l'animal sur le cốté droit, et de la soudure dubyssus aux corps étrangers.

Telles sont les propositions quil faut démontrer.

Quand on étudie la manière dont vivent quelques autres Mollusques, les Arches par exemple, on ne tarde pas à se convainere qu'ils peuvent s'enfoncer dans les rochers où ils sont fixés, en y adhérant avec une force extrême à l'aide d'un byssus très puissant dont ils sont pourvus. Ce byssus, dur, cartilagineux à son extrémité, est assez développé pour que le bord libre des valves présente une échancrure qui lui livre passage. L'Arche se tient toujours de champ; elle n'est pas couchée sur le côté; mais que l'on suppose l'animal placé exactement dans les mêmes conditions, sauf la position, c'est-à-dire que la puissance de son byssus reste la même, et qu'il soit couché sur le côté, on comprendra 
bien vite quelles modifications entrainera ce léger shangement de position. Il semble hors de doute que sur l'Anomie, e'est ce qui arrive; un byssus aussi très puissant s'est fixé solidement aux corjus voisins, et l'animal, au lieu d'être vertieal, est sur le côté. Cette seule condition biologique peut expliquer toutes les anomalies; et quand en commenegant je disais que, daus les Poissons, la position forcée sur le côté entrainait après elle la forme qui a servi à caractériser le groupe des Pleuronectes, ce n'élait pas sans raison que je cherchais à faire un rapprochement; et je dirai maintenant que l'Anomie est III Pleuronecte des Lamellibranches. En employant ce mot, je n'entends toutefois faire aucune comparaison entre des êtres si différents; et je veux seulement faire comprendre ma pensée, sans chercher à appliquer d'une manière absolue à un animal toujours fixé le nom d'un animal qui est libre.

Et d'abord, l'ossicule est un byssus.

L'étude comparative du byssus dans les Lamellibranches prouve que cet organe est placé le plus généralement en arrière du pied. Ainsi dans la Nacre (1), la Monle (2); la Dattile (3), ete., on le voit sorlir d'une fente dont les lèves se prolongent jusqu'aux bords d'une cavité creusée à la face postérieure de l'organe de la locomotion (4).

Dans l'Anomic (5), ce rapport est exactenent le mène. On a vu le pied rejeté un peu à gauche, mais on distingue très nettement qu’il est antérieur à l'ossicule.

Le cercle que forment les lèvres et les brauchies en se réunissaut par leurs extrémités enferme toujours et le pied el le byssus; cela est très distinct ici.

Sil'on pénètre plus avant diuns l'intérieur de l'organisme, et que l'ou cherehe à avoir des preuves tirées de la position et des rapports avec le système nerveux, voici ce que l'on observe : Le ganglion huccal droit, très facile à reconnaître avec son homologue de

(1) Pinna notilis.

(2) Mytilus edulis

(3) Lithodome, Modiolu lithophaga.

(4) La Lima squamosa fait exception, le Byssus est en avant.

(5) Pl. J, fig. 6 . 
gauche, et les deux ganglions pédieux, forment un cercle où la bouche seule se trouve enfermée (1); le pied et le byssus sont en dehors. Ainsi rien de différent ici entre l'Anomie et les autres Acéphales.

D'après la position respective du pied et du byssus, c'est à la base du premier que doivent être les ganglions pédieux. C'est, en effet, ce qui s'observe : les nerfs des museles qui meuvent l'un et l'autre de ces organes naissent des centres nerveux qui nous oceupent. L'Inomie ne fait pas encore exception à la règle; les nerfs animant les museles de son ossicule viennent du ganglion pédieux droit, ainsi que du ganglion branchial. On voit, en général, dans les Acéphales, que les muscles postérieurs du byssus recoivent chacun un nerf, qui remonte des ganglions branchiaux sur la ligne médiane. Cela paraît très nettement dans la Moule; ici l'importance de l'un des museles de l'ossicule explique le volume de la branche nerveuse qui remonte vers ce faisceau musculaire. Aussi, loin de trouver là une différence, ne doit-on y voir qu'une preuve de plus à l'appui de l'opinion que je cherche à démontrer.

Ainsi la position de l'ossicule est bien celle ru'oecupe habituelleunent la partic ì laquelle nous la comparons, et je erois surtout que les rapports avee le système nerveux ont une grande valeur, et montrent bien quelle est son origine. Du reste, cette détermination d'un organe d'après la connaissance des filets nerveux qu'il reçoit n'est pas sans exemple en anatomie comparée; et l'une des plus jolies applications est sans contredit celle qu'en a faite M. Blanchard, (quand il a montré la nature de quelques appendices des Goléodes. Il a fait voir, en effet, que, malgré la forme, on devait les considérer comme ayant telle ou telle origine, d'après les filełs nerveux qui se rendaient dans leur intérieur; car jamais le centre cérébroïde, sus-cesophagien, ne fournit des nerfs aux mêmes parlies que les ganglions sous-œesophagiens. On voit que e'est par une marche analogue que l'on arrive à donner des preuves de la véritable nature de l'osselet des Anomies.

La dureté, la consistance pierreuse el caleaire, peuvent-elles 
s'opposer à ce que l'on voie dans cet ossicule une production semblable au byssus? Je ne le pense pas. D'abord il est des exemples où la consistance devient des plus grandes : dans les Arehes, on a beaucoup de peine à diviser le byssus, tellement les fibres en sont serrées et agglutinées par une matière plastique, et même à l'extrémité adhérente aux corps étrangers, il devient presque impossible de les couper. Mais dans le cas qui nous oceupe, on remarque que la partie, même la plus dure, est finement striée, ce qui indique une origine fibreuse; et quand on arrache l'ossicule de ses insertions musculaires, on voit une surface hérissée de pointes, qui rappelle. alssolument la même disposition que l'on observe dans l'Arche, quand on détache brusquement aussi le byssus de ses museles.

En général, le développement des faisceaux musculaires est toujours en rapport avec celui de l'organe qu'il est appelé à mouvoir. Dans l'Arche, souvent prise comme terme de comparaison, les paruets de muscles ont de telles proportions, qu'ils s'étendent de la partie antérieure à la partie postérieure; mais on remarque toujours qu'ils sont en dedans des muscles adducteurs des valves. Dans les Jambonneaux, dans les Moules, ete., on les voit avec une nelleté qui en rend l'étude très facile. Ils se divisent en deux masses, l'une antérieure, l'autre postérieure, ce qui fait en tout quatre museles. Voisins de ceux du pied, ils se mêlent et se confondent quelquefois avec eux, comme on le voit pour la Moule.

Dans l'Anomie, il y a une grande différence; on ne trouve que deux masses (1), toutes les deux d'un même côté. On a vu qu'elles sont parfaitement distinctes du musele des valves, et que les opinions des auteurs, qui regardaient les museles de l'ossicule comme une dépendance de ceux-ci, n'étaient pas exactes. Des deux masses, l'une est antérieure, l'autre est postérieure. Faut-il admettre qu'elles correspondent aux deux museles antérieurs et postéricurs gauches du byssus ? La première est formée de faisceaux beaucoup plus petits que la seconde, absolument comme cela se remarque dans un même musele des valves (Limes, Peignes, etc.); elle semble plus tendineuse, et c'est sur elle que s'attache plus spécialement l'ossi-

(1) PI. I, fig. I $(x x)$. 
cule ; enfin la veine branchio-cardiaque en fait le tour en passant en avant. Pour ces raisons, on peut considérer le byssus comme n'ayant (fu'un seul muscle. D'ailleurs, quand on étudie le pied, on voit qu'il a pour se mouvoir deux petits faiseeaux musculaires : l'un, postérieur, se confondant avec la masse dont il vient d'être question; l'autre, antérieur, sortant au bord antérieur du manteau (1); mais ce dernier est accompagné par quelques paquets de fibres veuant du byssus, qui se confondent eneore avec lui, et représentent, je crois, à la fois un des muscles antérieurs peu développés du pied et du byssus. Cette différence dans l'appareil moteur de l'ossicule ne peuten rien infirmer l'opinion qui est soutenue ici ; on en trouvera l'explication dans ce qui reste à étudier.

D'après les détails qui précèdent, il ne paraît pas possible de méconnaître l'origine de l'ossicule; mais il reste maintenant à montrer comment la soudure et l'ossification du byssus, aiusi que la position forcée de la coquille, peuvent expliquer toutes les anomalies dont il vient d’être ruestion.

Les Mollusques à byssus flexible se fixent en restant souvent couchés à plat, comme, par exemple, les Peignes bigarrés (Pecten varius), la Moule comestible (Mytilus edulis), etc.; mais si le byssus est résistant, on les voit se poser de champ comme l'Arche Noé (Arca Noé, A. barbata), ete. Que l'on suppose la première position coïncidant avec la condition de résistance, et l'on verra bientôt, par les progrès du développement, le manteau et la coquille, du côté où reposera l'animal, arrêtés dans leur développement en un point qui correspond juste à l'obstacle que leur oppose l'ossicule. Bien certainement la perforation, qui semble être au centre de la valve droite, a dû être d'abord une simple échancrure du même bord; elle a été peu à peu transformée par l'accroissement des parties qui se sont pour ainsi dire rejointes antour du byssus qu'elles n'ont pu repousser, et qu'elles ont contourné. Ceci se comprendra d'autant plus, que la déviation a certainement porté d'abord sur le manteau qui, flexible et mou, a pu céder ; mais comme e'est lui qui séerète la coquille, il lui a imprimé sa forme.

(1) Pl. I, fig. I (v). 
Ce premier résultat est suivi d'une sorte de transport sur te côté droit de tous les organes; mais, par opposition, les muscles du byssus de ce côté deviennent inutiles : aussi les voit-on avorter complétement; de plus, toute la force de développement se porte sur un seul fuisceau, sur celui qui doit remplir le rôle le plus aclil et le seul nécessaire. Son action est d'autant plus efficace, qu’il devient perpendiculaire au levier qu'il doit mouvoir' c'est ce qui l'a rendu parallèle au musele des valves, et l'a fait prendre quelquefois pour une partie de celui-ci. Ainsi les muscles, venant s'attacher à la valve droite, n'ayant plus de raison d'être, avortent. Rapprocher du corps sur lequel est fixé l'animal la valve gauche ou supérieure, tel est le seul but du bysaus; aussi ne trouve-t-on qu'un muscle qui suffit à cet unique mouvement, c'est le postérieur. L'antérieur, confondu avec ceux du pied, est à peine marqué; ainsi s'explique cette masse si robuste d'un seul muscle, dont la direction a changé, et dont le développement a élé la conséquence du but que lui impose la position biologique nouvelle.

La présence au milieu de l'organisme de ce gros faisceau museulaire, l'avortement des autres, le changement dans sa direction, tout contribue à porter une perturbation dans l'ordre habituel des appareils.

Ainsi le raceourcissement de la branchie droite est évidemment le résultat de la présence, en avant de son extrémité, d'un corps résistant qui l'empêche de s'étendre.

La position de la bouche sur le côté droit est la conséquence: d'une part, de la position reculée de l'extrémité antérieure de la branchie, à laquelle doivent s'unir les lèvres; de l'autre, de l'allongement de la branchie gauche, et enfin de la torsion générale qui a tout rapporlé ì droite; de plus, en se tournant ì droite, le byssus a dû laisser le pied un peu du côté opposé, ce qui nous explique le voisinage et presque la fusion du ganglion buceal gauche ct des ganglions pédieux.

Quant à la position bizarre du cour, sur laquelle des détails nombreux ont été domnés, est-il possible de la rapporter au développement et à la position du byssus? C'est plus difficile, mais cependant l'explicalion qu'on jeut en domer ne nuanque pas de vrai- 
semblance. D'abord une partie considérable de la glande génitale, et le cecum, fort long, se trouvent accolés surle lobe droit dumanteau, unis cncore au corps proprement dit par des ponts qui s'étendent de l'un à l'autre : on dirait qu'il y a eu transport des glandes sur le côté, vers lequel tont l'organisme s'est tourné; le cocur aurait ćlé abandonné, et laissé seulement enfermé dans son péricarde, (qui, n'étant plus soutenu par les organes environnants, se serait accolé au ventricule et aux oreillettes. Cette sorte d'abandon de la masse centrale par une grande partie des glandes explique comment l'abdomen se trouve pour ainsi dire réduit au foie seul; comment, en avant du muscle des valves, on ne retrouve plus cette masse abdominale, presque toujours si nettement caractérisée dans les Peignes, les Spondyles, les Bucardes, etc.

La position de la branchie, interposée entre la partie glandulaire, le cœecum dans le manteau et la masse du foie, ne peut infirmer cette idée; car, d'après des recherches faites sur l'embryogénie des Houles comestibles, l'appareil digestif se développe bien avant les organes de la respiration, et l'on comprend comment alors le cœcum a pu se placer dans le manteau, et comment plus tard les organes génitaux en se développant ont dù s'étendre dans cette partie, en se glissant à côté de l'appendice du tube digestif. Il est impossible, en effet, de ne pas reconnaître que tout l'animal s'est porté du côté droit; et l'explieation qui vient d'être donnée, pour paraître un peu forcée, ne me semble pas moins exacte.

Comment se rendre compte de ce dernier fait, le cour n'est pas traversé par le rectum? C'est ce qu'il est très difficile d'expliquer par les changements de position. Est-ce une exception, conséquence, ainsi que la brièveté du tube intestinal et la longueur dı coecum, des anomalies, déjà si nombreuses et plus importantes, ayant leurs causes dans les changements de direction du byssus? C'est probable, mais ancunc particularité anatomiçue n'en donne la preuve absolue.

En résumé, l'Anomie est un être très irrégulier ; mais toutes les déformations qu'elle présente sont la conséquence dı changement de direction de son byssus, qui prend une consistance osseuse en se fixant invariablement aux corps étrangers. Ces conditions 
toutes particulières s'accompagnent de changements dans la position des organes et surtout dans le développement des parties, qui prennent des proportions en rapport avec le rôle nouveau et le but ‘fu'elles doivent remplir.

L'Anomie ne diffère donc pas au fond des Acéphales lamellivranches; elle est formée sur le même plan général qu'eux, et les variations, qui, au premier abord, sont difficiles à reconnaitre pour des modifications des formes ordinaires, trouvent toutes leurs explications dans les particularités qui se rattachent à l'origine et au rôle de l'ossicule dont la nature semblait avoir été méconnue.

Ces détails suffisent pour montrer qu'il n'est pas possible de considérer, avec Bruguières, l'Anomie comme un Acéphale multivalve; avec M. Deshayes, comme un genre intermédiaire aux Brachiopodes (Térébratules) et aux Acéphales lamellibranches; ou enfin de voir, avec d'autres auteurs, dans l'ossicule une partie de la coquille, véritable opercule mû par uneportion du muscle des valves.

J'espère par d'autres recherches démontrer encore ces vues, et faire voir que l'embryogénie, ici comme toujours, est consultée avantageusement pour résoudre des questions souvent difficiles et quelquefois insolubles sans son secours.

\section{EXPLICATION DES FIGURES.}

Planche 1.

\section{Organisation de l'Anomie (Anomia ephippium).}

Fig. 1. Animal enfermé dans le manteau; on remarque quatre muscles du côté gauche.

Fig. 2. Id., vu du côté droit. Ossicule $\left(x^{\prime}\right)$; muscle des valves $(y) ;(z)$ orifice où passait le ligament élastique de la charnière.

Fig. 3. Coupe de l'animal, pour montrer les glandes génitales mâles ou femelles, suivant le sexe $(s, s, s)$, accolées sur le lobe droit du manteau, au milieu desquelles se place le cœcum $(q)$. Le rectum $(l)$, très court, naît à côté du précédent de la cavité stomacale $(j)$ creusée dans le foie $f .\left(u, u^{\prime}\right)$ points d'union des glandes génitales placées dans le manteau avec les parties qui restent sur le foie.

Fig. 4. Le manteau a été enlevé, ainsi que l'ossicule et lo pied, afin de mettre en 
évidence les rapports des lèvres qui entourent la bouche $(b)$, et qui vont s'unir aux extrémités des branchies ; $(h)$ feuillet externe supplémentaire.

Fig. 5. Les branchies écartées laissent voir les corps de Bojanus $(r r)$, dont les orifices $(c)$ sont en dehors des ganglions branchiaux $(g)$ : de ceux-ci part un nerf du manteau $(m)$; lobe droit, des nerfs branchiaux $(n)$ un gros nerf du muscle du byssus (o); ganglion buccal droit $(i)$.

Fig. 6. (b) bouche; $(i)$ ganglion buccal droit; id., gauche; $\left(p^{\prime}\right)$ pied; $(p)$ ganglions pédieux.

\section{PLANCHE 2 .}

Fig. 1. Le cœur et les vaisseaux du côté droit. (V) ventricule placé en dehors de l'économie, non traversé par le rectum; (OD) oreillette droite ; (BC) vaisseau branchio-cardiaque droit.

Fig. 2. Idem du côté gauche.

Fig. 3. Circulation veineuse. Le sang veineux, avant de se distribuer aux branchies, arrive par deux vaisseaux (SV), l'un à droite, l'autre à gauche, placés à la base du repli falciforme qui porte les branchies, se répand dans ce repli, est reçu dans un nouveau sinus $\left(S^{\prime}\right)$, d'où il sort pour aller dans chaque filament en passant par une petite ampoule allongée : cet ensemble de conduits forme une sorte de corps spongieux.

Fig. 4. Une partie de ce corps spongieux grossi.

Fig. 5. Circulation artérielle. Il semble y avoir un bulbe aortique $(a)$ d'où partent les artères antérieures $\left(a^{\prime}\right)$ et les postérieures $\left(a^{\prime \prime}\right) ;\left(b c^{\prime}\right)$ vaisseau branchio-cardiaque gauche; $(e)$ vaisseau rapportant au cœur le sang veineux qui ne passe pas par les branchies.

Fig. 6. Texture du corps de Bojanus. Grossissement, 400 diamètres.

Fig. 7. Animalcules spermatiques et corpuscules qui les produisent. Grossissement, 500 diamètres.

Nota. - Dans les deux planches les lettres sont les mêmes pour les mêmes organes; cela a dispensé de multiplier les renvois.

PARIS, - Imprimerie de I. MARTINET, rue Mignon, 2. 


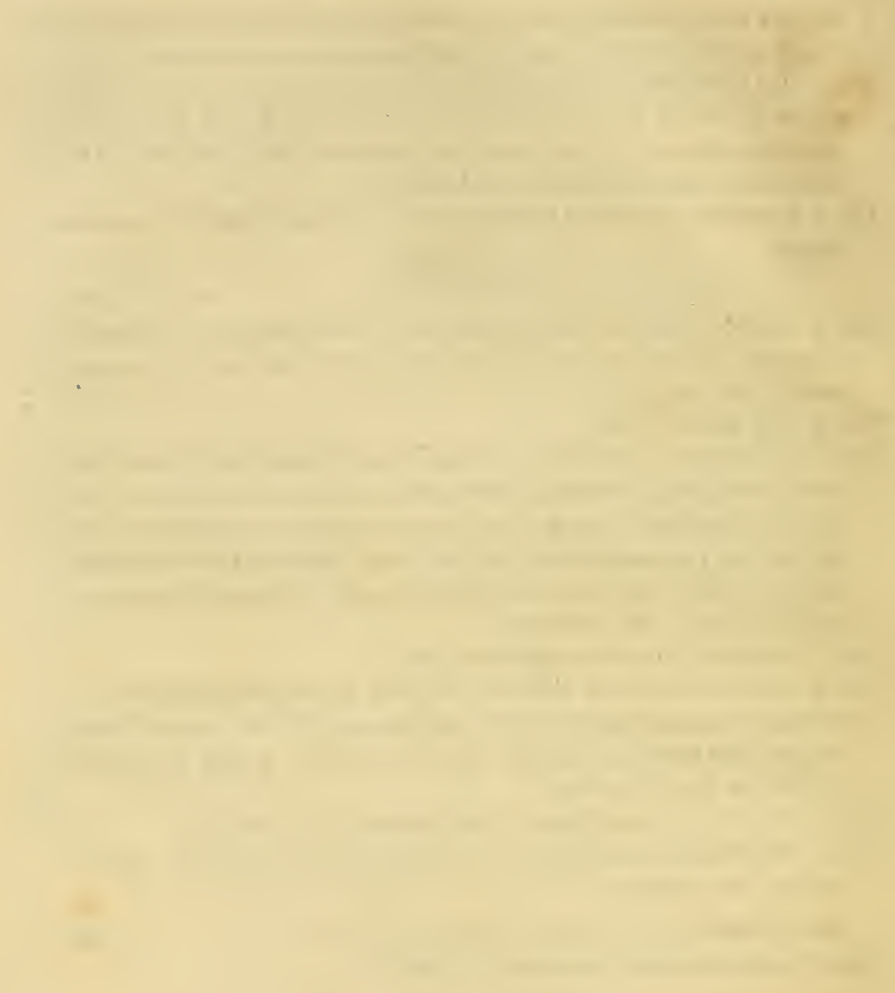



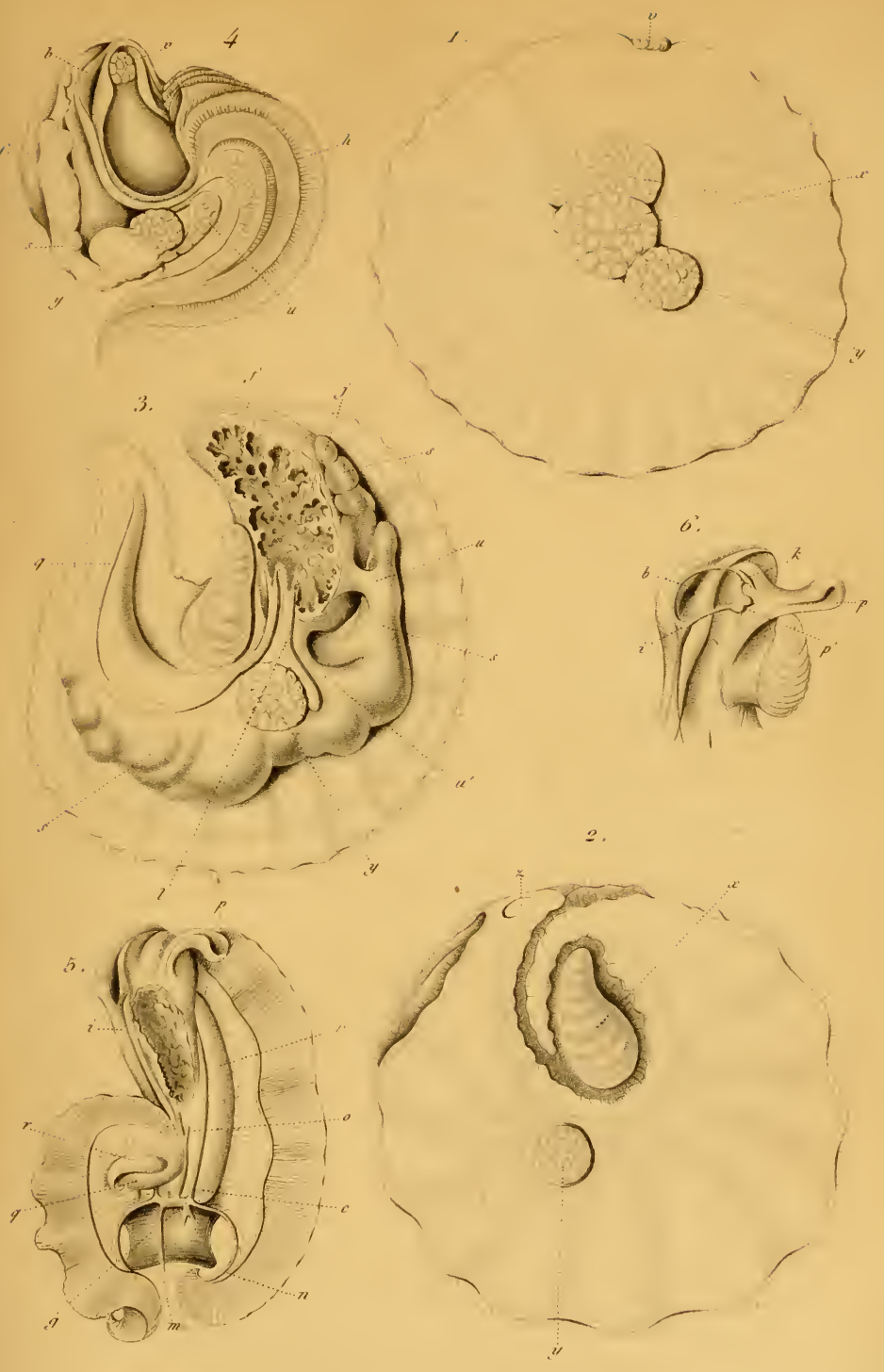

Digarnisntion de I'Anomie. 


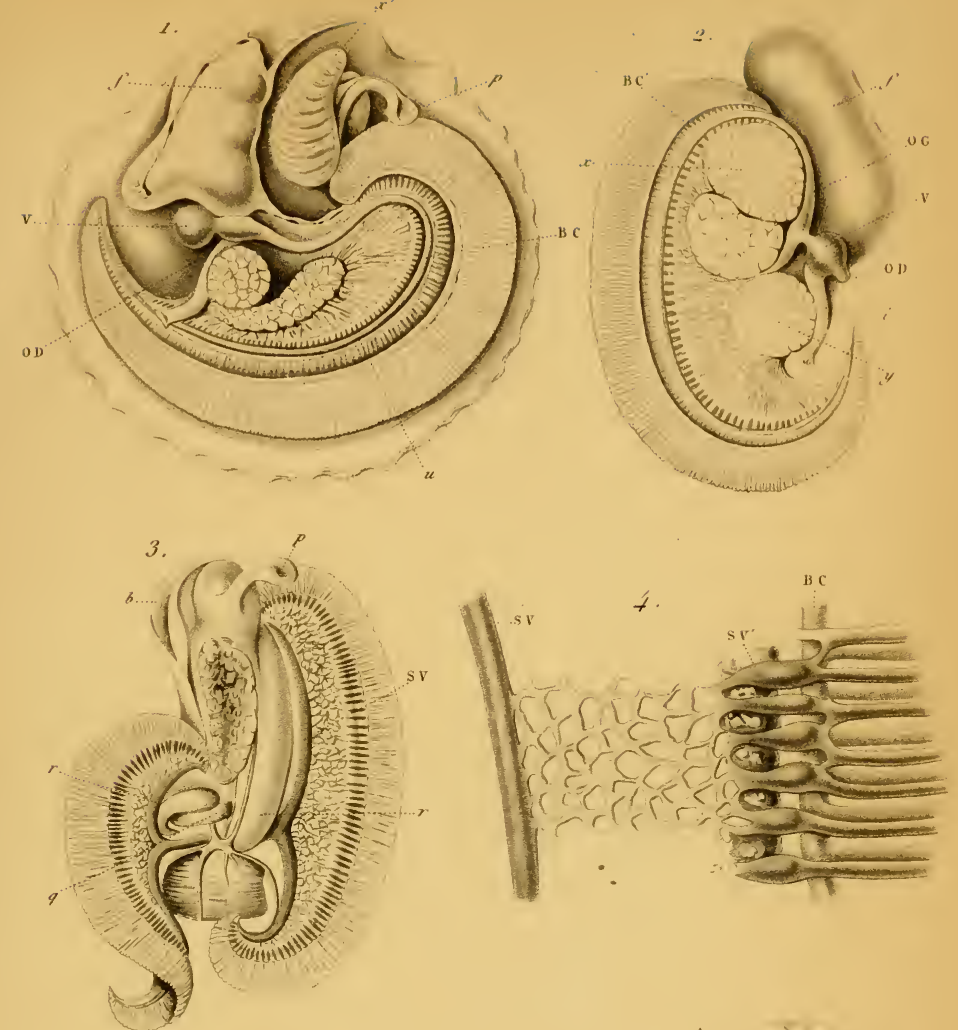

O3)

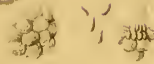
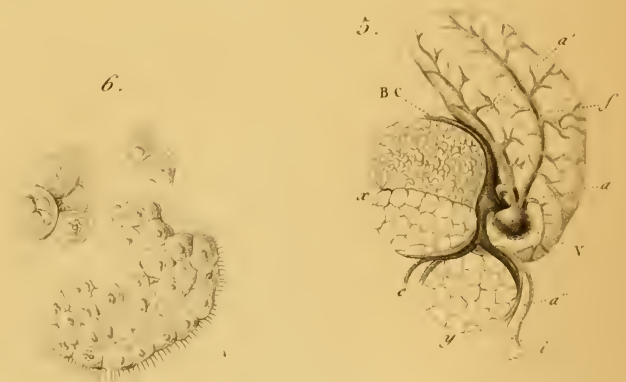

Oiganisation de I'Anomie 

RECHERCHES

SUR LES

\section{ORGANES GÉNITAUX DES ACÉPHALES LAMELLIBRANCHES.}

\section{Introduction.}

L'histoire de la reproduction du groupe nombreux des Mollusques acéphalés lamellibranches n'a pas été l'objet d'études suivies, bien que les questions qui s'y rattachent aient donné lieu à des discussions fréquentes basées toutes sur des opinions absolues, nées de l'observation d'un petit nombre de faits trop vite généralisés. Quand on consulte les auteurs, on est frappé du désaccord de leur manière de voir ; mais on ne tarde pas à s'apereevoir que la cause des contradictions, sans cesse renaissantes, se trouve dans le manque de recherches générales faites sur un nombre suffisant d'espèces. On voit surtout que les naturalistes, imbus de cette idée que la connaissance de l'un des types d'un groupe suffit pour avoir une idée du groupe entier, se sont trop hâtés de généraliser. Aussi les opinions, quelquefois exactes quand il s'agissait d'une seule espèce, sont-elles devenues souvent fautives quand on les a étendues à un grand nombre.

En abordant la question, on se trouve done en face des opinions les plus opposées, soutenues tour ì tour, après avoir été abandonnées ou reproduites, tantôt dans les traités généraux, tantôt dans des monographies. C'est surtout dans ces dernières que l'on rencontre la vérité sur l'espèce dont il y est question; tandis que dans les travaux sur l'enscmble de l'organisation des Acéphales, on voit s'accumuler les erreurs. Cela montre que la cause des divergences des opinions est bien celle qui vient de lui être assignée. 
A l'origine de la science paraît d'abord la croyance aux générations spontanées. D’après Aristote, les coquuilles naissaient dı limon de la mer. On cite cette opinion, mais on ne la diseute plus aujourd'hui ; il eût été même inutile d'en parler si Bonanni (1), à la firı dı xvı' siècle, ne l'eût reprise, et, en agitant la question de savoir si les coquillages bivalves tirent leur origine du limon des eaux, n'eût été, par la publication de son livre, cause des recherches de Leuwenhoeck.

Qu'il soit permis de faire remarquer combien, ì toutes les époques, la génération spontanée n’a servi qu'i déguiser l'ignorance des naturalistes. Elle explique le développement de tous les animaux dont la présence dans une localité embarrasse. D’abord ce sont les Anguilles elles-mêmes qui naissent de la vase des marais, puis les Vers; bientôt la connaissance plus exacte des organismes fait perdre à cette théoricue et gratuite explication une partie de son importance. Aujourd'hui elle ne s'applique plus qu'aux êtres rudimentaires, et sur lesquels nous ne sommes pas encore complétement éclairés; elle n’est, en effet, soutenable et soutenue que pour les Infusoires.

Peu satisfail des opinions de Bonanni, Leuwenhoeck 2 chercha comment se reproduisaient les Acéphales; et pour cela, il étudia, avee raison, d'abord les sexes. Dans ses leftres, il indique nettement que dans les Moules, les Anodontes, les Mulettes, quelques Vénus et Buccardes des côtes de la Hollande, les glandes sexuelles sont séparées et portées par des individus différents.

Voilà la première observation exacte basée sur l'examen mieroscopique, seul moyen de pouvoir résoudre la question.

Cependant Méry (3) et Poupart (4) faisaient aussi, au commen-

(1) Recreatio mentis et oculi in observatione animalium Testacearum, ed. latin. Rome, 1684 .

(2) Leuwenhoeck (Antoine), Arcana naluree detecta, 1722, t. II, epist. 83, p. 417 ; et t. III, epist. 95 et 96 .

(3) Mèry (Jean), Remarques failes sur la Moule des élangs (Anodonte) (Ac. des sc, 1701$)$.

(4) Poupart (François), Remarques sur les coquillages à deux coqnilles, el premièrement sur les Moules (Anodontcs) (Ac. des sc., Paris, 1'706). 
cement de a sibele, des recherehes sur les Anodontes, et ils arrivaient ì des conclusions tout opposées. Ils admettaient l'hermaphrodisme.

La question, laissée de còté jusqu’au milieu du xrü siècle, est alors tirén de l'oubli par Baster 1), dont les observations, publiées à Harlem, sur les œufs et la semence de quelques plantes ou animaux marins, fout reconnaitre un observateur exact. On aurait tout lieu de s'étonner que les résultats de ses expérienees, empreintes du eachet de la vérité, n’aient pas suffi pour convainere les naturalistes; mais on voit ceux-ci, toujours poussés par des idées préconcues, nées de faits biologiques embar rassants, être constamment à la recherche de la démonstration de l'hermaphrodisme. Baster observa des Moules comestibles (2) placées sćparément dans des rases distinets, et il remarqua que les unes émettaient un liquide blanchâtre formant un muage dans l'eau, et fourmillant d'une multitude de petits Vers comme des Anguilles, tandis rue les autres rejetaient de très petites Moules. Sa conclusion fut que les sexes étaient séparés, que les premiers étaient des mâles et les seconds des femelles.

Ces faits semblent concluants; cependant Poli (3), dans son grand et heau travail sur les Mollusques des Deux-Siciles, revint de nouveau à la réunion des sexes sur un même individu; et l'importance de son ouvrage, les développements qu'il lui donna, les détails qu'on y rencontre, déterminèrent Jongtemps les naturalistes à en admettre les résultats sans vérifieation. L'opinion du savant italien a prévalu dans les ouvrages classiques, c'est à elle qu’il faut attribuer cette idée très généralement répandue encore aujourd'hui que les Acéphales se fécondent eux-mêmes, idée qui est reproduite par Cuvier même dans son Règne animal.

Ainsi, avant le $\mathrm{xix}^{\boldsymbol{\theta}}$ siècle, on voit Leuwenhoeck et Baster soutenir la distinetion des sexes, tandis que Méry et Poli arlmettent

(1) Baster (Job), Opuscula subseciva, continentia observationes miscellaneas de animalculis et plantis quibusdam marinis eorumque ovariis et seminibus, $2 \mathrm{vol}$. in-4. Harlemi, 1759-1765.

(2) Baster, loc. cit. De mylilis, t. I, liber III, p. 101.

(3) Poli (Xavier-Jos.), Teslacea utriusque Sicilirp Paric 1791-179:3 
l'hermaphrodisme; entre ces deux derniers auteurs, il faut faire une distinclion : le premier pense yu'un seul individu ne peut suffire, tandis que le second eroil que le fluide mâle peut féconder les oufs sur le même animal.

Il est curieux de voir que la vérité se trouve toujours du côté des auteurs qui ont fait usage du microscope. Aujourd'hui on comprend très bien la supériorité des observations faites à l'aide de cet instrument; mais on ne peut se rendre compte des raisons qui déterminaient l'opinion des auteurs, ne prenant pas pour point de départ de la distinction des sexes, l'œuf et le spermatozö̈le, seuls éléments capables de fournir des idées netles sur la question.

A cefte époque, c'est-ì-dire ver's la fin du xwm siècle, paraît une opinion étrange, qui, bien qu'un peu en dehor's de la question, s'y rattache cependant soit par les recherches nouvelles qu'elle fait entreprendre, soit par la critique qu'elle renferme de la nature de la branchic externe des Naidées, prise par Méry (1) pour une partie de l'organe génital : je veux parler de la détermination, sous le nom de Glochidium parasiticum, des jeunes larves de ces animaux comme un parasite par Ratlie 2 ).

Assez longtemps après celte prétendue et singulière découverte, Prévost de Genève 3), roulant s'assurer du fait, étudia la reproduction des Mulettes des Peintres. Les expériences de cet habile physiologiste sont trop remarquables et trop concluantes pour ne pas les rapporter. Il examina d'abord les glandes, et ne les trouva jamais remplies à la fois de spermatozö̈les et d'cufs ; toujours ces éléments étaient portés par des individus distinets. Ce premier fait anatomique aurait déjà pu suffire pour faire ouvrir les yeux aux naluralistes séfluits par les travaux de Poli ; mais Prévost ne s'en contcnta pas, il le confirma par des expériences phỵsiologiques d'une rigueur complète. Il plaça isolément dans des baquets des mâles et des femelles, et remil mnsemble d'antres individus de sexes

(1) Méry, loc. cit.

(2) Ralke, 1797

(3) Prévost, De la géu'ration des Moules des peintres (.tan. des sc. natur., fre série, t. VII, P. 449. - Mémoires de la Socieli de physique el d'histoire naturelle de ficuice, 1. III, $182 . \%$. 
dilférents. Il vit dans les premiers ses Mulettes rejeter, les unes, un fluide blane linteux rempli de spermatozoïdes, les autres des unfs restés inféconds, tandis que lì où étaient réunis les deux sexes, les crufs se développèrent. Élait-il possible de montrer plus nettement que ces Mollusques ne pouvaient se suffire, que leurs sexes étaient séparés, et que la fécondation ne devait s'opérer que par le voisinage des milles et des femelles? On a de la peine à comprendre qu’il ait été tenu si peu de compte de recherches aussi rationnelles, dirigées avec autant de soin, à une époque où les études physiologiques étaient loin d'être ee qu'elles sont devenues aujourd'hui.

Ces faits infirmaient les opinions de Ratke. Cependant Jacobson (1), deux annćes plus tard, en 1827, les renouvela dans un mémoire conmuniqué à l'Aeadémic des sciences, et sur lequel de Blainville lit un rapport (2). Ce fut une oceasion pour eet académicien de s'occuper du sexe des Mollusques lamellibranches, et bien qu’il eut connaissance du travail de Prévost, il se rangea cependant à l'opinion de Poli; mais en admettant l'hermaphrodisme, il pensa que la glande mâle devait être plus extérieure que l’ovaire, afin que les ceufs en li traversant pussent être influencés par le lluide spermaticue. L'auteur dı rapport ne fit pas connaître les faits anatomiques qui déterminaient son opinion, où l'on a peine à voir autre chose qu'une simple vue de l'esprit. Il critique même indirectement le travail de Prévost, en lui reprochant de n'avoir pas indiqué comment it reconnaissait les mâles des femelles. Le physiologiste de Genc̀ve ne pouvait faire un parcil oubli, il aurait trop donné prise à la eritique; aussi a-t-il indiqué dans son mémoire (3) que de légères blessures faites à l'abdomen des Mulettes, sans troubler les fonctions, lui permettaient, d'après la présence des oufs ou des spermatozoüdes, de distinguer les sexes.

Ainsi, à cette époque, la question n’a jas fait un pes. Le travail remarquable de Prévost n'a servi à rien, et l'on est de nouveau reporté au xvu siècle; les noms seuls sont changés : Baster est

(1) Jacobson, Oliservations sur le développement prélendu des oufs des Moulettes on Unios el des .1nodontes dans leurs branchies (Amn. des sc., 1827).

(2) Rapport de de Blainville sur ce mémoire

(3) Prérost, loc cil. 
remplacé jar Prérost, Poli par de Blainville, el Ralke par Jacobson.

Les ćtudes anatomiques sont même faites avee si peu de suite, que Tréviranus 1 admet que la sortie des arufs a lieu par la bouche, landis que Carus $(2)$ pense que e'est par l'anus.

1 mesure que l'on se rapproche davantage de l'éporpue actuelle, on ne trouve pas plus d'aceord : ansi de Baer / 3 avait pleinement confirmé les faits avancés par Prévost; mais II. Raspail (4), dans un travail commmiqué à l'Aeadémie, les réfuta, en objectant que les prétendus spermalozoüdes n'étaient que des portions d'épithélium vibratile.

Beaucoup plus frès rle nous, dans une série d'articles, justement estimés, sur l'anatomie des Lamellibranches, Garner (5) revient ì l'hermaphrodisme; il admet un mélange complet des glandes; il peuse que les cenl's sortent le l'ovaire dans des conditions convenables ì leur développenent; il nie mème l'existence d'un organe mâle distinet. On voit que l'hermaphrodisme ne peut pas être mieux indiqué $(6)$

II. Milne Edwards (7), ayant éludié à Nice les organes génilaux des coquilles de Saint-Jaeques, montra que, avee des glandes sexuelles distinctes, il y a cependant un hermaphrodisme bien marqué. Le savant professeur reconnut de plus à eette époque que

(1) Treviranus, Ueber die Zengung der Mollusken, recueil de MM. Treviranus et Tiedenann, vol. I, cah. 1, p. 31.

(2) Carus, Lehrbuch der Zootomie, p. 618, vol. ii.

(3) Baer, Frorieps noliced., janv. 1826.

(4) Raspail, Histoire des Alcyonelles des élangs, lue à l'Académie des sciences le 24 septembre 1827 .

(5) Garner, On the anatomy of the Lamellibranchiata conchifereus animals, t. II. Magazine of natural history de Charlesworth.

(6) Id., p. 439. " But there appears every reasons to believe that there is no $n$ difference in the individuals as to sexes and that the ova are discharged from $n$ the ovaries in a state fit to develope, wittout the necessity of the contact of " any vivifing fluid; or in other words that they are fecundated before they leave ") the ovaries, by lestes which must be conjointed with those organe. No distine, " male organes appears to be present. ")

(7) Milne Edwards, Observations sur la structure el les fonctions de quelyues Zoophyjtes, Mollusques el Crustacés de's cóles de la France (Ann. des sc. wat,

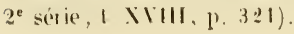


les Venus el les Cardium avaient, au contraire, les sexes séparés et portés par des individus différents.

Von Siebold (1), en 1837, établit la séparation des sexes dans plusieurs espèces. Depuis, ce savant a étendu cette observation à un plus grand nombre (2). Quant à l'hermaphrodisme des Peignes, sans le nier, il dit qu'il n'apu confirmer le fait observé par M. Milne Edwards, et cela paree qu'il a examiné une autre espèce (3).

Dans ses Lecons d'anatomie comparée, R. Owen (4) indique, d'après les observations des naturalistes, que les vues de Leuvenhoeck sont exactes; lui-même a pu s'assurer de leur vérité sur les Anomia qui se développent aux plages de l'Angleterre; mais il signale l'exception des Cyclas, où, suivant Wagner, un testicule s'ajoute à l'ovaire. Il est évident que le savant professeur n'a pas poussé très loin ses recherches, car il parle du testicule de l'Huître en décrivant la glande mâle; ce qui montrerait qu’il croit ì la séparation des sexes dans ce Mollusque, surtout si l'on remarque qu'il a cité l'exception découverte parWagner.

Je suis forcé de passer successivement en revue les opinions émises isolément sur quelques espèces, et ne portant que sur l'hermaphrodisme. Cette énumération, tout aride qu'elle puisse être, montre, je l'espère, les doutes qui existent encore sur la question; elle fait voir la nécessité d'une étude générale, d'un travail servant à fixer lesidées, en faisant dans les travaux antérieurs la part du vrai et du faux.

II. Van Beneden (5) en appelle de l'opinion de Leuwenhoerk sur les Anodontes; il rejette la séparation des sexes : car " c'est „ bien, dit-il, l'hermaphrodisme le plus complet quel'on connaisse ; n à mesure qu'ils se forment, les oculs et les spermatozoïdes vien"nent au contact, et la fécondation s'opère (6). "Nous aurons occasion de revenir sur cette manière de voir.

(1) Voy. Siebold, Muller's Archiv., 1837, p. 380.

(2) Voy. Siebold, Anat. compare, trad. franc., t. I, p. 286

(3) Idem, I I, p $28 \%$, note 8

4) R. Owen, Leclures on the comparative anatomy and physiology, t. I, F. 28 7.

(3) Van Beneden, Bultelins de l'Academie de Bruxelles, t. XI, nn 11, 1844.

(6) Llem page 381 . 
M. de (Quatrefiges (1), en étudiant le genre Taret, a montré l'erreur où Sellius et M. Deshayes sont tombés en considérant eet animal comme hermaphrodite; les résultats que lui avait fournis l'anatomie ont été confirmés par des observalions physiologiques de fécondation et de développement. Tous les faits, qu'une longue étude m’a permis de constater, se rapportent complétement à ceux que le savant aeadémicien a cousignés dans son remarquable travail.

Dans les additions que M. Duvernoy (2) a ajoutées à la seconde édition des Lecons d'anatomie comparée, on trouve que "les bivalves hermaphrodites sont probablement les plus nombreux. " Les quelques renseignements qu'on y rencontre conduisent l'auteur à celte conclusion très juste: "Les organes des deux sexes existent toujours réunis ou séparés."

Les Huitres à elles seules out donné lieu à plus de discussions que tous les Acéphales réunis. Il est préférable de renvoyer l'appréciation des opinions an moment où nous nous occuperons de ces Mollusques. Qu'il nous suffise de dire maintenant qu'ils ont été considérés tour à tour coinme à sexes distincts ou comme hermaphrodiles.

Il est un dernier travail sur lequel l'attention doit se fixer plus spécialement, e'est la deseription des Mollusques de l'Algérie par M. Deshayes (3). Il forme la collection la plus nombreuse des monographies anatomiques les plus étendues, que l'on ait sur les Acéphales. Le mode de publication, les magniliques planches qui l'accompagnent, en font un ouvrage de luxe; on avait tout lieu d'espérer que la question des sexes s'y trouverait montrée sous son véritable jour; il faut avouer ecpendant (ju'il n'en est rien, et que l'hermaphrodisme parait y ètre considéré comme la condition sexuelle générale des Acéphales. Cette opinion, quoiqu'elle ne soit nulle jart formulée nellement, ressort cependant de l'ensemble du travail ; aussi est-il nécessaire de quelques développements jour

(1) De Quatrefages, Ann.des sc. natur., $3^{\text {e }}$ série, 1849. Le Tarel, son histoire et son ieveloppement.

(2) Cuvier et Duvernoy, Leçons d'analomie comparee, t. VIII, p. 496, 498.

(3) Exploration scientifique de I'Agerie, publiée par ordre du gouvernement français, Mollusques, par M. Deshayes 
montrer qu'elle est bien telle que nous l'indiquons. Dans les premières monographies, on voit l'auteur encore à la recherche de faits qui démontrent la réunion des sexes; maisàmesure que l'on avance, et que les observations se multiplient, la question est moins doutense. Ainsi II. Deshayes, ayant reẹu des Solen vivants (1), espérait trouverdans l'ovaire des preuves de la fécondation en y découvraut à la fois des oufs et des zoospermes; mais son " atteute a été " trompée, l’ovaire n'ćtait pas assez mùr.... Nous avons également " cherehé, dit-il, dans l'organe spécial des crochets, si nous y dé" couvririons des animalcules spermatiques, destinés ì produire "plus tard la fécondation des œufs. Nous n’avons pas été plus heu"reux (2). "

Dans les Pandores, on trouve décrites les parties mâles et les parties femelles : “ Des organes particuliers, assez nombreux, font ") une sorte de bordure, à la masse viscérale et au foie en par" ticulier, le long du bord dorsal, depuis le muscle adducteur ") antérieur juscu'ì l'ovaire, en suivant les anfractuosités ocei„ sionnées par la charnière (3). " En examinant au microscope, une portion mince de cet organe, on reconnait des granules qui a se prolongent en une quene courte et très pointue, que „les organes dont il s’agit sont de véritables testicules, et que, dans " les Pandores, les deux organes de la génération sont nette" ment séparés. Au reste, ees organes milles oceupent la place qui » est affectée ordinairement à l'organe des crochets, lequel, conme "nous l'avons vu, joue un rôle évident dans l'acte de la généra" tion (4). " Plus loin, à propos des Lyonises, après avoir dit qu’il n'avait pu rencontrer les organes mâles analogues ì ceux des Pandores, il ajoute : «Il est done à présumer que ces deux sortes d'or"ganes génitaux sont confondus, comme cela a lieu si souvent chez " les Mollusques acéphales (5). „On voit déja se traduire l'opinion

(1) Un grand nombre de recherches de cet auteur, comme il l'indique luimème, ont été faites sur des animaux conservés dans l'alcool.

(2) Loc, cil., p. 152.

(3) Loc. cit., p. 250

(i) Loc. cil , P. 250 .

(:) Loc. cit, P 270 . 
sur l'hermaphlurodisme ou sur le mélange des glandes des deux sexes.

Dans les Lutraires, l'organe descrochets reparait, et son voisinage avec l'ovaire conduit à conclure que ses globules rougeâtres doivent se répandre dans la glande femelle, et y joner un rôle important pour les fonctions de reproduction.

Les Mésodesmes n'ayant présenté que des animaleules spermatiques, l'ovaire est considéré comme vidé, et l'organe " mâle est "probablement confondu avec l'ovaire, car nous y avons observé " un grand nombre d'animaleules spermatiques. „Et puis, dans les conclusions, résumant tout ce qui a été dit sur le genre : "L’organe » des erochets ne paraît pas exister dans les Mésodesmes. Ce genre „ serait done du petit nombre de ceux qui jouissent du singulier pri"vilége d'avoir les organes des deux sexes confondus en un $n \operatorname{seul}(1) . "$

Ainsi déjà il ressort de ces faits que lorsque les organes des crochets existent, M. Deshayes les considère comme des glandes mîles ; que lorsqu'au contraire ils ne paraissent pas, les testicules et les ovaires ne font plus qu'un.

On trouve dans la monographie dı dix-septième genre quelques figures d'une grande exactitude et d'une vérité remarquable, les ovaires surtont y sont parfaitement représentés; mais quant à l'interprétation des faits, elle est toujours dinns le même sens. Ainsi les organes des erochels out présenté encore des granules pourvus d'appendices candiformes extrêmement courts, qu'on ne peut affirmer être des spermatozoïdes, parce que les individus sur lesquels ils ont été pris étaient morts. a Au reste, en examinant l'ovaire sur » des animaux vivants, nous n'y avons jamais rencontré de sperma„ tozoïdes avant l'époque de la fécondation, ce qui nous porte à con» clure que l'organe des erochets doil être considéré comme l'or" gane mâle dr la génération $(2)$. "

tinsil'on voit parces citations que e'est toujours la mème mamicure de voir : ynand l'organe des crochets existe, il uy a pas d'animalcules spermatiques dans l'ovaire, séparalion des glandes portées par

(1) Loc. cil., p. 400 .

(2) Loc. cil., P. 498 
un mêne individu; quand cet organe n'existe pas, Jrésence du lestienle an milieu de l'ovaire, fusion des deux glandes.

Dans les conclusions, l'opinion est plus nettenent exprimée : " Les organes de la génération sont de deux sortes chez les Lavi"gnons : un ovaire énorme envahissant toute la masse abdominale, " et un organe mâle placé sur le dos, et remplissant la cavité des " erochets $(1) \ldots$. . "

“.... Nous ignorons ì fuel moment sont disper'sés dans l'ovaiue " les spermatozoüdes accumulés dans l'organe des erochets $(2)$. "

Il ne peut paraitre douteux maintenant que M. Deshayes considère le's Acéphales lamellibranches commedes êtres hermaphrodites ; ear on voit partout les efforts qu'il fait pour retrouver les glandes des deux sexes sur un même individu, et partout on le voit conclure ì leur fusion, quand il ne peut les rencontrer; que s’il n'a pas la preuve de la fécondation, comme nous l'avons dit en commençant, en trouvant dans l'ovaire les deux éléments caractéristiques des sexes, il croit que l'époque de son observation n'est pas favorable, et rue les éléments ne sont pas arrivés à leur maturité. Il m’a paru utile de montrer en détail cette opinion, parce qu'un ouvrage aussi sérieux porte par cela même un plus grand trouble dans la question en montrant tous les Acéphales hermaphrodites, et en attribuaut à un organe, dout la présence est loin d'être constante pour l’auteur lui-mème, un rôle qui ne lui appartient pas. Aussi est-il évident yue la glande mâle ayant été complétement méconnue, toutes les opinions sur le sexe entachées d'inexactitude ne peuvent plus soutenir la eritique. Cela est si vrai, que des dessins remarquables l'exécution et de vérité, désignés comme représentant les ovarres en voie de développencut, ne sont autre chose que l'image fidèle des testicules gorgés de liqueur séminale (3).

(1) Loc. cit., p. 506 .

(2) Loc. cit., p. 507.

(3) Dans la planche $\mathrm{L}$, on trouve une figure qui représente l'ovaire lorsqu'il est encore rempli de a cette matière blanche semblable à de la laite de poissons : " la forme des vésicules ressemble à des digilations inégales ou obtuses, ou plu" lôt à une multitude de coecums (p. 463)... n Quand l'ovaire est mùr (pl. I.II, fig. 6), on voit que ces granules qui le forment "sont euchainces les uns aur 
Je dois en terminant citer une note toute récente de $\mathbf{~ I I}$. Humbert 1) sur la structure de la glande de diverses espèees du genre Pecten. L'auteur observe que les unes sont hermaphrodites, les autres à sexes séparés; il s’attache à concilier ainsi les opinions, en apparence opposées, de MII. Edwards et Sicbold. Du reste, l'anatomie des organes n'y est pas complète, car l'auteur avoue n'avoir pu reconnaitre les canaux excréteurs.

En résumé, on voit d'abord la génćration spontanée eacher l'ignorance des naturalistes; puis viennent les opinions qui montrent les femelles pondant des oufs aptes par eux-mêmes à se développer, et n'ayant pas besoin de l'influence d'un fluide mâle; plus tard paraît l'hermaphrodisme avec ou sans distinction des glandes; enfin la connaissanee de la séparation des sexes n'est aequise qu'au moment où le mieroscope est appliqué à l'étude de la question; mais loin de la résoudre, ee moyen d'investigation ne fait que renouveler et multiplier les discussions; il les rend plus ardues en fournissant des faits plus diffieiles à constater. En général, l'apparition d'une opinion opposée à celle que l'on avait déjì est la conséfuence de la manière trop absolue dont la première était présentée, et surtout de sa généralisation à toutes les espèces, quand elle n'en avait le plus souvent qu'une seule pour point de départ; l'absence de travaux généraux, faits en s'aidant des moyens d'investigations sulfisants, est done une cause des erreurs répétées dans les ouvrages les plus importants jusqu'ì notre époque; en sorte quill n'existe pas de question plus obseure et moins facile à éclaircir en s'en fenant aux recherches bibliographiques. On voit de plus que toujours on n’a agité que cette unique question : les Acéphales sont-ils hermaphrodites ou à sexes séparés; qu'on a étudié d'une manière pen approfondie la strueture ot les autres caractères des glandes génitales.

Tel était l'état de la question, lorsque j'ai entrepris les recherches qui font l'ohjet de ce travail. Il était naturel de faire des efforts pour éviter les causes qui paraissaient avoir produit les erreurs

1) autres... quiils sont fixés aux parois d'un raisseau, ce qui leur donne beau1) coup de ressemblance avec une grappe de rasin (p. 46:3). D

(1) Humbert, Amo dessc, nut., $3^{\mathrm{e}}$ scrie, rol. XX, 1853. 
nombreuses. Dans ce but, et afin de pouvoir présenter avee plus de confiance les résultats de mes observations, j’ai cru devoir observer, à des époques varićes de l'année, dans des localités différentes, le. plus grand nombre d'espèces possibles. Aussi ai-je passé une partie du mois d'août 1852 à la Rochelle, tout l'été, et une partie dı printemps de l'annéc 1853 en Espagne, tant à Barcelone, sur Ie continent, que dans les îles Baléares, à Palma et à Mahon. J'ai fait surtout dans cette dernière localité de nombreuses et précieuses observations. Pendant le mois de septembre de la même année, eı explorant le littoral méditerranéen français à Marscille, aux Martigues, sur l'étang ou la petite mer de Berres, à Bouc, à Cette, j’ai recueilli des faits importants.

Poursuivant toujours le mème objet, j’ai, en 1854, passé durant le mois d'avril quelque temps aux environs de Bordeaux, on j'ai étudić la Mulette des rivières; à Rochefort et à la Rochelle, oì j’ai pu avoir des espèces nouvelles, et constater encore, dans une autre époque, les résultats obtenus une première fois deux ans auparavant.

Dans les mois de mai et de juin 1854, j’ai étudié les Acéphales des environs de Paris, et ceux appportés des ports de mer de la Nanche, sur les marchés de la capitale.

Enfin, dans l'été et l'automne de cette même année, j’ai, en explorant les eôtes de Normandie, ì Bernières, Courseulles, Grandville; et celles de la Bretagne, à Saint-Malo, Saint-Servan, SaintJacut-la-Mer, les Hébiens, eu l'oceasion de recueillir quelques faits de plus.

Ainsi les recherches ont été faites à des époques ef dans des mois différents sur des points éloignés. La concordance des résultats permettra, je l'espère, de pouvoir désormais se faire une idée plus exacte des organes reproducteurs du groupe nombreux des Acéphales lamellibranches.

Árant de conmenerr l'étude des organes de la génération, pour bien s'intendre ef éviter toute confusion, il me parait nécessaire de caractériser le terrain qui va servir aux olsservations, et de dire quelyues mots très brefs du plan général d'organisalion d'un . Iré- 
phale lamellibranche, pour fixer la valeur des termes mis en usage dans le courant du travail.

Le corps d'un Acéphale lamellibranche est composé de deux parrties similaires, placées sỵmétriquement de charque côté d’un plan vertical passant par la bouche et l'anus. Les irrégularités et les différences qui peuvent exister sont toutes accidentelles, et n’infirment en rien ce principe.

Pour poser l'animal, on place en haut la charnière de la cofuille, en bas le bord libre, et en avant l'extrémité qui rorrespond ì la houche. Dans cette position, le Mollusfue a un côté droit, un côté gauche, ete., ete. Il est important de se rappeler que e'est à cette position que seront rapportées toutes les directions.

En dedans des branchies, que protégent le manteau et la coquille, parait le corps proprement dit; sa forme est variable; on le trouve plus ou moins aplati, globuleux ou allongé; il se termine en avant près de la bouche. Celle-ci est recomnaissable à sesfeuillets labiaux qui revìtent un peu l'apparence générale des branchies.

On trouve fréfuemment sur le bord inférieur et antérieur $1 \mathrm{~m}$ appendice museulaire moteur, le pied, qui porte souvent un byssus. Le foie est placé près de la bouche, et l'intestin se contourne en arrière de lui vers la base du pied. Nous domnerons désormais le nom de masse viscérale ou d'abdomen à cette portion du corps qui renferme les viscères. Souvent l'abdomen se contourne en arrière et en dessous du muscle postérieur des valves; conme dans les Peignes, il prend la forme de la bosse de Polichinelle, et ressemble à un pied; mais on ne doit pas le confondre avec cet organe.

Les muscles rétracteurs du pied traversent la masse splanchnique; les postérieurs, en se reudant près du muscle postérieur des valves, se dégagent dans le voisinage de celui-ci, et semblent faire 111 pédoncule à l'abdomen; entre autres excmples où cela est surtout évident, on peut citer les Cardium.

Entre le muscle postérieur des valves, d'une part, le lobe dorsal du foie ot la masse viscérale, de l’antre, il y a une sorte de vide, de dépression oecupée par le eceur ; clle se prolonge latríalement sous l'insertion des bramchies, ef loge l'organe dre Bojanus. 
Dans le système nerveux, un seul point doit fixer l'attention. (On sait que les trois paires de ganglions buccaux, pédieu.x, branchicux, sont réunies par des connectifs; l'un d'eux, le connectifbucco-branchial, affecte un lapport inmédiat avec les organes génitaux. Ce rapport est constant ; il conduit à reconnaitre ef i fixer la place des orifices de la génération. J'appelle done l'attention sur lui.

On adunet, en général, daus l'appareil de la reproduction, trois ordres d'organes, suivant qu'ils produisent l'ćlément essentiel, qu'ils fournissent des liquides accessoires, ou qu'ils aident l'action des fluides mîles sur les germes; cesont les glandes génitales proprement dites, les annexes ou les appendices copulateurs.

Daus les différents groupes du rìgne animal, la constance el la perfection des deux derniers est en raison directe de la perfection de l'ètre. Ainsi, pour ne nous occuper que des Iollusques, on voit les Gastéropodes présenter des organes copulateurs et des annexes remarquahlement développés, tandis que dans les Acéphales tout l'appareil est réduit aux glandes sexuelles seules; et e'est à peine si l'on peut regarder un organe placé dans leur voisinage comme jouant le rôle d'annexe : mais l'organisation des premiers est bien supérieure ì celle des seconds. Il y a rertainement des exceptions : presque toujours alors des conditions particulicres viennent en donner la raison.

On ne doit done pas s'attendre à rencontrer dans les Acéphales lamellibranches des fonctions multiples et complexes concourant ì la reproduction de l'espèce, les organes étant rédnits, pour ainsi dire, ì leur plus simple expression, ì la partie fondamentale.

Pour se conformer à l'usage, il fant étudier successivement les appareils femelles et les appareils mâles; mais une difficulté se présente tout d'abord, car on rencontre l'hermaphrodisme au sujet duquel on a vu tant de discussions s'élever. Aussi, anticipant sur ce qui sera démontré plus loin, est-il nécessaire d'étalılir quelques distinctions qui permettent de faire rentrer les descriptions anatomiques daus les formes habituelles.

Tantôt les sexes, portés par des individus différents, sont séparés, tantôt, an contraire, ils peuvent ittre rémis sur un sẹl animal, M être distimets ou eonfoundus. Les Lamellibranedes qui présenient 
la première disposition doivent être nommós diöqques, tandis qur les seconds méritent à tous égards le nom de monoüques. Dans eenx-ci il fant eneore faire une distinction secondaire on rappont avec la séparation on le mélange des gylandes. On peut done élahlir les divisions suivantes :

Les Acéphales lamelli-
branches sont $\left\{\begin{array}{l}\text { diö̈ques } \\ \text { ou } \\ \text { monöqques. Ceux-ci ont les glandes } \\ \text { sexuelles }\end{array}\left\{\begin{array}{c}\text { distincles } \\ \text { ou } \\ \text { confondues. }\end{array}\right.\right.$

Ces expressions expriment très nettement les différents ćtals des sexes dans ce groupe; leur usage fréquent daus les sciences naturelles dispense de plus d'explications.

\section{ORGANES GÉNITAUX PROPREMENT DITS.}

I.

Acéphales dioĩques.

$\S I^{\mathrm{er}}$.

Organes femelles (ovaires).

L'ovaire des Acéphales dioünues varie beancoup avec le moment de l'année où on l'examine, l'âge et l'espèce de l'animal. C'est ì cela que tiennent les changements de couleur, de forme, de sitnittion et de rapport qui n'ont eertainement pas contribué peu à faire tomber les auteurs dans une série d'erreur's que nous anrons soin de relever dans le courant du travail. La ponte surtout change eomplétement l'aspeet de l'abdomen. Ainsi, tandis yue daus les Peignes bigarrés (1), les Clovisses (2), l'Huitre vermeille (3) et la plupart. des autres espèces, on reneoutre des individus dont la masse viseérale est prespue globuleuse, d'autres, an contraire, présentent leur abdomen aplati, lanelliforme, et l'on peut distinguer, an travers des téguments, les circonvolutions intestinales ot les museles du pied. Du reste cela n’a rien qui puisse élomner, quand on sait

(1) Peclen varius.

(2) Venus decussuta.

(3) Spondylus grederopus. 
quelle prodigieuse quantité d'oufs est pondue par une seule femells.

Il semble que les jeunes Acéphales, toute proportion gardée, ont une glande génitale moins considérable. Ainsi le nombre des lobules glandulaires est d'autant plus grand que l'animal est plus fros; il n'augmente pas proportionncllement à l'accroissement du corps, il le dépasse ; relativement, l'animal âgé pond beaucoup plus a'œufs que le jeune, car une surface de un centimètre carré, par exemple, renferme peut-être deux fois plus d'acini dans le premier que dans le second. Aussi les études anatomiques sont möins difficiles à cause de la distinction plus grande des éléments sur les individus de petite taille.

I.e volume de l'ovaire varie essentiellement avec les espèces: cela n'est pas douteux, mais c'est difficile à préciser; d'abord parce que la taille des individus n'est pas la même, et que l'on vient de voir des variations causées par l'âge. Cependant, d'une manière générale, on peut dire que plus la glande ovarique s'avance sur le foie, et plus elle acquiert de développement. Ainsi dans la Lime (1), où l'ovaire enveloppe tous les organes, à l'exception du manteau, des branchies, du pied et des voiles labiaux (2), l'animal paraît complëtement rouge quand il est en gestation ; moins la couleur, les Pholades sont dans le même cas, deurs glandes génitales prennent un développement excessif. Dans la Nacre(3), bien que l'ovaire ne s'ẻtende pas sur le foie et en soit parfaitement distinct (4), il forme une masse considérable. Dans l'Huître vermeille (5), il est aussi très développé, et je crois qu'on peut le considẻrer comme relativement plus volumineux que dans les Bucardes, ete. (6).

La position, on le comprend, doit se ressentir des variations de volume. Elle est caractéristique surtout dans les jeunes, et c'est sur de jeunes Peignes bigarrés (7) et des Lucines (8) que l'on pent fa-

(1) Lima squammosa.

(2) Pl. 7, fig. 1.

(3) Pinna nobilis.

(4) Pl. 5, fig. 1 et $2(0)$.

(5) Spondylus gaderopus.

(6) Cardium edule, Cardium ruslicum.

(7) Pecten varius.

(8) Lucina lactea. 
cilement s'en rendre compte, ainsi que dans la Bucarde rustique, si facile à disséquer. Dans la Lucine, le pied très long se détache nettement de la masse viscérale, et permet de constater, ainsi que dans les Bucardes, que l'ovaire ne pénètre pas dans son intérieur, comme on l'a trop souvent dit. La glande occupe la partie du corps qui se trouve au-dessous du foie et du cour, en arrière de la bouche, au-dessus du pied et en avant du muscle postérieur des valves; elle entoure les circonvolutions intestinales.

Par les progrès du développement, les lobules se multipliant, on les voit remonter peu à peu sur les côtés du foie qu'ils finissent par recouvrir; il est même des espèces où les organes sont totalement cachés sous la couche glandulaire ovarique, comme cela s'observe dans l'Arche, la Lime, le Spondyle, les Pholades. Jamais on ne reneontre la disposition que j'indique ici dans les Jambonneaux (1). Le foie dans cette espèce forme une masse nettement séparée et antérieure à l'ovaire $(2)$.

Ainsi il est difficile d'assigner une forme spéciale à la glande génitale; on peut dire qu'elle occupe tous les espaces du corps laissés libres par les autres organes, et qu'elle s'étend et remonte davantage à mesure qu'elle est plus développée. C'est ainsi que dans les Huitres vermeilles (3) on la voil accompagner le rectım jusque vers l'anus, all-dessus du muscle postérieur des valves.

Ce qui précède suffit pour montrer que les rapports varient avec les conditions diverses; mais on peut cependant voir que la glande est toujours placée autour de l'intestin. Dans quelques cas, celui-ci s'en détache et s'en sépare avec une facilité remarquable. Dans les Cardium, les Vénus, les Pecten varius, par exemple, rien n'est plus facile à voir que les grappes de l'ovaire groupées autour des circonvolutions intestinales; mais dans l'Anodonte, pour peu surtout que la gestation soit passée, c'est un travail des plus pénibles que de chercher à isoler l'intestin; on n'y parvient qu'avec les plus grandes difficultés.

Dans l'Anomie et la Moule enmestible, l'ovaire oreupe une
(1) Pinna nobilis.
(2) Pl. 5, fig. 1 et 2.
(3) Spondylus grederopus. 
position toute différente; il est logé en grande partie dans l'épaisseur lu manteau, et par cela même affecte des rapports très différents. Dans l'Anomie (1), l'un des voiles du manteau seulement enferme tme partie de la glande; dans la Moule comestible, les deux voiles sont occupés entièrement par la presque totalité de l'ovaire (2). Nous aurons oceasion de revenir sur cette disposition, anornale dans la première espèce, naturelle dans la seconde.

On peut consulter avec beaucoup d'utilité les magnifiques planches qui accompagnent les ouvrages de Poli (3) et de $\mathbf{Y}$. Deshayes (4) pour toutes les généralités qui touchent à la position, au volume, aux rapports, etc., de l'ovaire; mais il faut se tenir en garde contre la confusion que ces auteurs ont faite entre l'ovaire et le testicule. Le travail de Garner (5) renferme aussi à cet égard beaucoup de renseignements.

Il est un autre caractère de l'ovaire sur lequel il faut s'arrêter à cause de son importance, et surtout à cause des erreurs commises ¿̀ son égard. Sa couleur, en effet, est tantôt blanche, tantôt jaune, et tantôt enfin d'un rouge plus ou moins vif, plus ou moins caractéristique. La première teinte s'observe nettement, parce qu'elle tranche sur la teinte brune du foie, dans les Bucardes (6), les Clovisses (7) ou Vénus, les Peignes bigarrés, les Lucines (8), les Pholades (9), les Rupicoles (10), les Gastrochènes (11), les Lavignons (12), les Prères (13), etc. Cependant pour être complétemeut

(1) Anomia ephippium.

(2) Pl. 6, fig. 2 .

(3) Poli, Testacea utriusque Sicilice.

(4) Deshayes, Exploration scientifique de l'Algérie, Mounusques.

(5) Garner, On the anatomy of Lamell, conch.; Transact. of zool. Soc. of Loudon, II, 1841, ou dans le Mag. de Charlesworth, t. II

(6) Cardium rusticum.

(7) Venus decussata.

(8) Lucina lactea.

(9) Pholas dactylis.

(10) Petricola striata.

(11) Gastrochana tarentina.

(12) Trigonella piperata.

(13) Carbula striatu. 
Jans la vérité, je dois ajouter que dans quelques individus, des Peignes, des Lucines, des Lavignons, etc., la teinte passe un peu au jaune, mais très légèrement. Dans les Unios des peintres la couleur jaune est bien sensible, mais elle est faible. Dans lesChames elle devient jaune-bistre pâle ; dans le Solen (Solen vagina) elle est brun-chocolat, quelquefois un peu vineuse; tandis que dans les Dattiles (1) elle est fauve ou rouge-brique mêlé de jaunâtre ; dans la Moule comestible, elle est d'un rouge de litharge; dans la Nacre, cette teinte se rapproche du rouge-brique foncé, devient presque vineuse dans la Lime, et l'Arche de Noé; enfin dans le Spondyle pied d'âne la couleur est magnifique : elle ressemble à celle du jus de la framboise, et elle a valu à cette espèce le nom d'Huître vermeille, d'Ostia vermella, qui lui a été donué par les Mahonais.

Ce caractère a une grande valeur pour faire recomnaitre les sexes, mais seulement après l'examen microscopique.

Poli semble avoir fait une étrange confusion : il a remarqué ces belles couleurs, et a surtout exprimé son admiration (2) à l'égard du Spondyle, mais il les a mal interprétées ; au lieu de les considérer comme le caractère des ovaires parfaitement développés, il a supposé qu'elles n'appartenaient qu'aux germes non encore arrivés à eomplète maturité, et il croit qu'au moment où les ceufs sont mûrs ils changent de couleur et deviennent blanes ; évidemment l'auteur a pris les mâles pour les femelles : cela résulte essentiellement, du reste, de sa croyance à un hermaphrodisme général (3).

(1) Modiola lithophaga.

(2) Loc. cit., t. 1I, p. 108 :

(3) T. II, p. 68, dans les géuéralités sur la couleur il s'exprime ainsi : " In ") diversis autem stadiis... tum colore admodum variare conspicitur... quibus 1) sensim a fœtu deductis, rosæus ipsorum color in aureum immutatur, donec " postremo ovis ad integram maturitatem perductis, ramuli prædicti ultra mo") dum distenti... promiscuam massam effingunt lacteo veluti humore turgidam ») lacteoque splendentem lacteus iste humor; ingentem veluti errorat ovulorum ") cumulum, quem in suo sinu fovet;... sunt quædam Testacea in quibus sub" fuscus est ovarii color, qui deinde opportuno tempore in albicantem illico immuv tatur. „ Et il cite les exemples où ce changement peut être observé facilement, la Moule comestible, le Spondyle, la Chame grypoïde, les Huîtres de Saint-Jacques, et higarrées, les Limes, les Pinnes nobles, etc, etc. - Ici le 
M. Deshayes admet comme Poli le changement de la conleur avec les progrès de la maturité des œufs ; pour lui, l'ovaire passe du blane au rouge. Quoique plus près de la vérité, il n'en reste pas moins dans l'erreur ; croyant à l'hermaphrodisme, il prend, à l'op)posé de Poli, le testicule pour l'ovaire non développé (1).

Il suffit, je crois, d'opposer ces deux opinions pour montrer combien cette idée préconçue de l'hermaphrodisme a servi à enbrouiller et à obscurcir la question. Certainement la vivacité de la couleur augmente à mesure que les germes s'approchent davantage du terme de leur maturité ; d'un autre côté, après la ponte, les ovaires pâlissent beaucoup ; mais je dois dire que dans des Pinnes marines, alors que les œufs n'étaient encore reconnaissables qu'aux vésicules germinatives, la teinte de l'ovaire était déjà très caractérisée. Aussi toutes les assertions sur le changement de couleurs doivent être considérées comme des erreurs résultant des idées fausses qu'avaient les auteurs sur les sexes. Faisons toutefois cette restriction, que lorsque la glande est complétement vidée, la teinte peut disparaître absolument, comme cela s'observe avec pleine évidence dans une espèce monoüque, le Pecten Jacobeus, où, comme on le verra plus loin, la partie femelle est d'un bel orangé avant que les culs soient sortis.

\section{Struclure.}

L'anatomie intime des glandes génitales est très difficile dans certaines espèces; aussi faut-il en commencer l'étude par des exemples convenablement choisis si l'on vent arriver à des notions mot illico semble indiquer que le changement est brusque, et certainement l'auteur a pris le testicule et les individus mâles pour des ovaires et des individus femelles.

(1) Mollusques de l'Algerie, t. I, p. 151 . "Nous avons eu l'occasion d'obser) ver dans le Solen legumen ce phénomène fort remarquable du changement de ") couleur de l'ovaire; avant que les œufs soient en état d'être pondus, l'ovaire 》) reste d'un blanc jaunâtre, tel que nous l'avons fait représenter (planches et „) figures du Solen legumen); les œufs étant complétement mûrs changent de cou" leur et donnent à l'ovaire une couleur lie de vin très prononcée. "II n'est pas douteux que le testicule n'ait été pris encore pour l'ovaire, comme cela ressort des descriptions des Mésodesmes (p. 400) et des Lavignons (p. 465). 
précises; et c'est peut-être pour n'avoir pas agi de la sorte que des auteurs sont tombés dans l'erreur. Le Peigne bigarré et les Bucardes, surtout la Rustique, se prêtent d'une manière remarquable à l'observation.

Sur les côtés du foie d'un Peigne bigarré de taille moyenne, orr voit de petits îlots de substance blanchre ou légèrement jaunâtre , disposés en grappes. Cela parait ì l'oxil nu, et devient bien plus évident sous la loupe, qui permet de reconnaitre des éléments parenchymateux glandulaires, groupés autour des dernières ramifications d'un conduit excréteur (1 .

Le parenchyme se présente, dans le point que nous avons choisi, c1 une couche mince, dont les éléments se distinguent facilement, ear le foie, par sa teinte obseure, forne un fond sur lequel ils se détachent parfaitement. Avec une étude attentive, on distingue sinns difficulté dans ces petites masses une quantité de grains devenus polyédriques par leur rapprochement, et auxquels je conserverai le nom d'acini, uniquement pour la commodité de la description, bien que ce mot ait perdu de sa valeur, et que les auteurs, Müller en particulier, rejettent absolument son emploi $(2)$. Ces grains ou acini présentent à leur centre une teinte plus foncée; ils sont nettement séparés par une ligne obscure, quoique très près les uns des autres. Il est facile de voir (3) qu’ils se gronpent en nombre variable autour des ramifications des conduits qui serpentent entre eux, et ‘u’ils prodtisent des lobules primitifs; que ceux-ci en se réunissant forment des lobules secondaires, constituant les lobes prineipaux; ct qu'enfin, à cause de ces dispositions, on doit placer l'ovaire des Lamellibranches parmi les glandes en grappes.

Les conduits sont très nettement dessinés. Dans les espèces dont il est iei question, on les observe entre les lobules primitifs dont ils sortent pour se réunir deux à deux d'une manière irrégulière, et pour former peu à peu des trones plus considérables dont la marche devient plus difficile à suivre, ear ils plongent dans la couche profonde de la glande, et se confondent par leur couleur, leur trans-

1) Pl. 6, fig. 1. - Pl. 8, fig. 1.

(2) Physiologie, traduclion framcaise de Jourdan.

(3) P'l. 6. fig. I. 
parence avec les filanents musculaires ou fibreux. J'ai été assez heureux pour rencontrer la glande génitale, dans un état tout particulier, sur un Peigne bigarré (1), et cet état m’a permis de pouvoir me former une idée exacte de la marche des conduits principaux que j'avais cherchés bien longtemps, et toujours, malgré les soins les plus assidus, avec un insuccès désespérant. Tout le parenchyme de la glande était réduit à de petites masses terminant les ramifications, dans lesquelles on ne distinguait plus d'acini ni de grains quelconques. Était-ce un parenchyme avorté avec des canaux excréteurs bien développés, ou bien était - ce une glande revenue sur elle-même après la ponte? C'est ce qu'il ne m'a pas été possible de décider. Toujours est-il que j’ai pu suivre les conduits excréteurs avec pleine certitude, et que j'ai remarqué qu'ils consistaient en deux trones principaux : l'un, antéro-supérieur (2), venant du lobe sus-hépatique; l'autre, postéro-inférieur (3), venant du lobe abdominal. Ces deux conduits, formés par la réunion successive des ramuscules des canaux des deux lobes, se confondaient dans un point très voisin de l'orifice (4) de la génération. Le trone unique n'avait pas $\mathbf{1}$ millimètre de longueur, il recevait quelques petits ramuscules des acin $i$ voisins; aussi ne mérite-t-il guère le nom d'oviducle.

L'orifice se trouve dans la cavité de l'organe spongieux (5), dans cette glande placée entre le cour, l'abdomen et le muscle postérieur des valves; mais ce qui arrive dans le Peigne varié, le Spondyle (6) ne se présente pas dans les Cardium, etc. Comme l'étude de cet orifice se lie intimement à celle de l'organe de Bojanus, nous renvoyons pour nous en occuper au moment où nous traiterons de ce dernier. Nous dirons seulement ici que l'orifice génital est toujours placé sur les côtés du corps, à la racine de l'abdomen, tantôt dans l'organe de Bojanus, tantôt aı sommet

(1) Pl. 8, fig. 1 .

(2) $\mathrm{Pl} .8$, fig. $1\left(a^{\prime}\right)$.

(3) Pl. 8, fig. I (a).

(4) Pl. 8, fig. 1 (b).

(b) Pl. 8, fig. $1(b)$.

(6) Spondylus grederopus. 
d'une papille confondı avec celui de cette glande; lantôt enfin il en est distinct et s'ouvre tout près. Son rapport avec le connectif bucco-branchial facilite sa recherche; il est toujours en dehors de celui-ci, et vers le point où le cordon nerveux plonge dans la masse viscérale.

Les exemples aussi heureux que celui que je viens de eiter sont rares, et quand on veut suivre les vaisseaux excréteurs, on éprouve dans quelques cas des difficultés insurmontables, si l'on ne prend beaucoup de précautions. C'est surtout en commençant la préparation qu'il est utile d'apporter tous ses soins; car pour peu que l'on déchire les tissus plus qu'il ne faut, l'on est bientôt au milieu d'une sorte de bouillie où l'on a peine à se reconnaitre; les difficultês tiennent, pour la plupart, à une délicatesse ct àune fragilité extrêmes des tissus : au moindre attouchement les acini se rompent, inondent la préparation de leur contenu, et voilent les conduits excréteurs, dont la transparence et le peu de résistance les fait échapper facilement à la vue. Les conduits excréteurs sont surtout évidents quand ils sont remplis d'oeufs; mais si on les onvre ils se vident, leurs parois s'accolent et ils disparaissent. On peut encore les découvrir par des injections; mais combien les précautions ne doivent-elles pas être minutieuses pour ne pas entamer les parois de l'orifice génital : car par la moindre déchirure tous les tissus s”injectent, surtout les vaisseaux sanguins; et l'on court risque de prendre ceux-ci pour les canaux excréteurs de la glande. Si j'insiste sur ce fait, e'est pour montrer que les auteurs ont pu facilement faire des erreurs; elles sont, en effet, nombreuses ì cet égard.

Dans les Bucardes et le Peigne bigarré, où les choses sont les moins difficiles à constater, on voit, de charque côté du corps, un orifice génitil, auquel aboutit un conłluit excréteur unique, formé par la réunion de deux trones principaux venant des lobes sushépatiques et abdominaux. It y a donc deux ovaires, placés symétriquement un de chaque côté; mais la distinction entre l'ovaire droit et l'ovaire gauche n'est pas aussi narquée que semble l'indiquer la description; les deux glandes, en effet, se mêlent et s'melıcrêtrent sur la ligne médiane, où il est impossible de reconnaitre les parlies gandules des parties droites. Il ne serait même pas 
impossible yu'il y eût communication entre les canaux de l'une et les conduils de l'autre; je n'ai pas cherché à constater ce fait, à cause de la difficulté des préparations.

Telle est la disposition principale qui peut servir de type, et à laquelle on peut rapporter les différences, qui sont pour la plupart des consé(juences des variations tenant au volume, à la position ou au développement.

Quand on sait où trouver une chose, la recherche en devient plus facile; aussi, malgré la difficulté, arrive-t-on à reconnaître que, dans les Spondyles (1), la Lime (2), le parenchyme et les canaux glandulaires présentent ì peu près la mème disposition, que l'organe de la génération s'ouvre dans le sac glandulaire placé à la base des branchies.

Dans les Arches (3), les Dattiles (4), les Moules (5), l'orifice génital est placé au sommet d'une papille vers le milieu de la longueur du corps. Les acini, très peu distinets dans les deux premières espèces, adhèrent les uns aux autres, et sont très difficiles à isoler.

Dans les Nacres (6), il existe pour les canaux excréteurs des différences marquées. L'orifice (7) est placé très bas presque sur la ligne médiane et en arrière; aussi les conduits (8) se portentils en avant et en haut pour se distribuer aux différentes parties de la glande. Il y en a deux, l'un supérieur, l'autre inférieur ; le dernier est le plus important. Le parenchyme glandulaire ne semble plus formé d'acini distincts; il paraît comme une masse compacte, dans laquelle on ne reconnaît que bien difficilement les éléments primitifs. Cependant, vers la partie supérieure de la glande, quand on a enlevé le tube digestif et le cœur, on trouve dans le fond de

(1) Spondylus gæederopus.

(2) Lima squamosa.

(3) Arca Noe.

(4) Modiola lithophaga.

(5) Mytilus edulis.

(6) Pinna nobilis.

(7) Pl. 5, fig. I (c).

(8) Pl. 5, fig. 4. 
la gouttière (1) qu'elle forme à ces organes une couche assez mince, qui permet de constater que les acini sont disposés le long des canaux excréteurs.

Dans les Mulettes (2), on distingue nettement les acini quand on ouvre l'abdomen; ils se présentent au moment de la ponte en forme de cœecums allongés, très évidents, et faciles à voir; souvent ils sont bilobés, et se continuent par leur extrémité adhérente avec les ramifications des conduits excréteurs, disposés comme dans les premières espèces, et venant s'ouvrir auprès de l'extrémité antérieure des branchies.

Dans les Lavignons (3), les Vénus (4), les Corbules (5), les Lutraires (6), etc., les acini sont très évidemment appendus aux dernières ramifications des canaux.

J'ai constaté une disposition analogue, avec des modifications insignifiantes, dans toutes les autres espèces objels de mes recherches; il est donc inutile de les passer toutes en revue.

L'Anomie et la Moule, dont l'ovaire est placé en grande partie dans le manteau, font exception à ce qui vient d'être dit. Cette disposition a été observée pour la Moule par Poli (7), Siebold (8) et Garner $(9)$. Ce dernier auteur a aussi considéré les Modioles et les Lithodomes comme étant dans les mêmes conditions; mais Sicbold remarque avec raison qu'il n'a rien vu de semblable dans le Lithodomus dactylis, et que, dans cette espèce, l'ovaire occupe l'abdomen; e'est aussi ce que j'ai toujours vu sur la Dattile de Mahon.

Dans la Moule, on voit les glandes occuper un peu les côtés du foie, et aussi ce rudiment de masse viscérale placé en arrière du

(1) Pl. 5, fig. $2(i)$.

(2) Unio sinuata, id. litloralis, id pictorum.

(3) Trigonella piperata.

(4) Venus decussala.

(ち) Corbula striata.

(6) Lutraria solenoides

(7) Loc. cit. Testacea utriusque Sicilia.

(8) Analomic comparée.

(9) On the anatomy of the Lamellitranchiata, loc. cit. 
pied; mais la majemre partie de la ghande est dans le manteau (1). Quant aux conduits excréteurs, ils sont tris visibles; seulement if est à craindre que l'on ait pris pour eux les vaisseaux sanguins. La confusion n'est pas possible, si l'on observe que ceux-ci se voient sur la face interue du manteau, tandis que les premiers ne se re. marquent que sur la face externe, sur celle qui est immédiatement appliquée contre la face interne de la coquille. Il est à peine besoin de préparations pour en voir les ramuscules. Nés dans toute l'éten. due du manteau, et réunis en trois troncs principaux, l'un antérieur, l'autre postérieur, le troisième médian, ils ne forment plus qu'un seul canal (2 qui s'enfonce dans la glande annexe, en quittant le manteau au bord antérieur dı muscle postérieur du pied; de là il se dirige vers la papille qui a été indiquée plus haut, et reçoit les conduits venant des portions glandulaires hépatiques et abdominales. Cette portion du tube excréteur est assez résistante pour pouvoir être séparée nettement des parties enviromnantes, et l'on pourrait lui donner ici assez justement le nom d'oviducte.

En raison de ces conditions, on peut dans cette espèce étudier avec beaucoup de facilité la disposition des acini, et Jeur réunion enl lobules primitifs et lobules secondaires.

Tels sont les éléments dont il nous reste à connaìre la texture; on comprend qu'ils ne sont pas entièrement libres, et qu'ils sont entourés par ras lisang formant une trame véritable autour d'eux. Cette trame produite par l'entre-croisement des lamelles des tissus fibreux ou musculaires, des vaisseaux artériels, ete., forme des aréoles, dans l'intérieur desquelles se logent les acini. Nous verrons, en nous occupant des Acéphales monö̈ques à glandes conlondues, que ces trabécules, ces parois aréolaires, ont été prises par M. Davaine pour une partie même de l'ovaire, mais que cela n'est pas, comme on peut l'observer dans l'Unio des Peintres,

(1) Pl. 6, hig. 2. - Jai fait une observation, malheureusement incomplète, sur une espèce de Moule d'un rose vif, vivant dans le sable des côtes de Bretagne. Le manteau ne renfermait pas la glande génitale qui occupait sa place habituelle, n'ayant pas conservé la coquille que j'avais ouverte sur la plage et и ayant pas trouvé d'autres individus, je n'oserais donner le nom de l'espèce.

(2) PI. 6, fig. $2(b)$. 
où les acini se détachent très facilement des parois de l'aréole qui les renferme.

\section{Texture.}

De même qu'il était utile de commencer l'étude de la structure de l'ovaire par les exemples les moins difficiles, les moins confus, de même pour la texture le choix est très important, car les difficultés augmentent encore. Quelle est la texture de l'œuf, des acini, du canal excréteur? Où et comment est sécrété le premier ? Quel rapport a-t-il avee les seconds? Telles sont les questions qui doivent nous oceuper maintenant.

\section{OEuf.}

Rien n'est plus facile à se procurer que l'œuf des Acéphales. Quelle que soit la disposition des glandes, en piquant la masse splanchnique, sans aucune précaution, dans un point quelconque, immédiatement, si l'ovaire est en état de gestation, il s'écoule un liquide coloré comme la glande, où l'on voit une immense quantité de germes à des états divers de développement. Si le moment de la ponte n'est pas très éloigné, on peut, à l'aide de légères pressions, faire aussi sortir les œufs par les conduits naturels, et j'ai même, en titillant les orifices des glandes annexes ou de Bojanus, produit je ne sais quelle sensation qui a détermine, chez l'Huître vermeille (1) et la Lime (2), une ponte presque naturelle. Mais le plus sûr moyen est de prendre un lambeau de la glande, on y trouve toujours des œufs à divers états.

L'euf a une forme peu variable. On ne peut en juger que lorsqu'il est pondu. Il est rond dans l'Unio (3), le Lavignon (4); ovale dans la Spondyle (5), la Lime (6) et l'Arche (7), etc. Ces différences peu sensibles sont d'une importance tout à fait

(1) Spondylus gæederopus.

(2) Lima squamosa, pl. 7, fig. 1 (c).

(3) PI. 7, fig. 41, 10, 9, 8

(4) Pl. 6, fig. 7 .

(5) Pl. 7, fig. 4.

(6) Pl. 7, fig. 2.

(7) Pl. 6, fig. \& 
secondaire; mais il est nécessaire de bien établir que l'œuf est ré. gulier, plus ou moins sphérique, quand il est hors de la glande.

Dans l'ovaire, il parait tout autre; il est pyriforme, et se termine par une sorte de queue plus ou moins allongée, dont tous les auteurs, ayant donné des figures des œufs des Lamellibranches, ont indiqué l'existence. D'après M. de Quatrefages (1), la queue est excessivement marquée dans le Taret, où, suivant ce savant académicien, les neufs ressemblent à des larmes bataviques. Pour M. Deshayes, cette forme est tellement absolue qu'elle le conduit à une théorie sur le développement. Dans un grand nombre de cas, elle parait ètre le résultat d'un effet d'endosmose; car lorsqu'on peut détacher les acini (2) sans les rompre, et les cxaminer entiers sous le microscope en les comprimant graduelle. ment, on voit que les aufs qu'ils renferment sont polyédriques. ou ronds (3), mais qu'ils n'ont pas une forme aussi pyriforme; sur les Lavignons, les Bucardes (4), les Mulettes (5), le fait que j'indique est parfaitement appréciable; mais il est un exemple où les effets de l'endosmose sont des plus remarquables, e'est dans la Gastrochcena tarentina. Les oufs, pris dans l'ovaire et mis dans l'eau, affectent, sous les yeux même de l'observateur, les formes les plus bizarres (6); ils poussent des prolongements au nombre de trois, quatre et même davantage, et deviennent souvent triangulaires. Si l'on ne suivait cette transformation de la forme première presque sphérique ou ovale (7), on pourrait être embarrassé ; mais il n'y a pas de doute possible. Nous verrons, du reste, qu'une autre cause peut être assignée à ce prolongement pyriforme, mais toujours il est augmenté, ou causé en partie par l'eau.

Le volume des germes est très variable, et les différences qu’ils

(1) Loc. cit., Ann. des sc. nat., Développement du Taret.

(2) Pl. 6, fig. 6 .

(3) Trigonella piperata, pl. 6, fig. 7.

(4) Cardium rusticum, C. tuberculatum, pl. 7, fig. 3.

(5) Unio pictorum, pl. 7, fig. 11.

(6) Pl. 5, fig. 5.

(7) Pl. 5, fig. 6 . 
présentent ne sont nullement en rapport avee la taille des espèces. Les œufs les plus volumineux que j’ai observés sont ceux de l'Unio littoralis (1) et ceux du Pectunculus pilosus (2). Dans la Gastrochoena tarentina (3), ils sont aussi gros que dans le Spondylus gaderopus (4), et ceux de la Lima squamosa (5) sont presque aussi considérables que ceux de la Pinna nobilis (6). Il ne faut pas attacher à ces différences plus de valeur qu'clles n’en méritent; mais on ne peut s'empêcher d'être étomné de la disproportion excessive qui existe entre les germes dles Mulettes des Pétoncles d'une part, et ceux des Jambonneaux d'une autre, dont la taille est infiniment plus considérable.

Il va sans dire que le volume varie avec le plus ou moins grand développement; et qu'il n'est ici question que de l'ouf mûr.

La composition de l'œuf des Lamellibranches est la même que dans les autres animaux; on y trouve une enveloppe vitelline, un vitellus, une vésicule transparente et une on plusieurs taches germinatives.

Enveloppe vitelline. Avec les espèces, et aussi avec le point où l'œuf a été recueilli, son enveloppe parait différente; ainsi, tandis que, dans les IIuîtres vermeilles, l'œuf ne paraît pas entouré par une membrane (7) quand il vient d'être pondu, chez les Unio, au contraire, dans les mêmes circonstances, on le croirait enveloppé par une capsule épaisse, qui rappelle absolument la zone transparente de l'œuf des animaux supérieurs. Cette apparence particulière, et qui a èté cause de certaine confusion, se présente aussi dans les oufs qu'on prend dans l'ovaire (8) des Cardium rusticum, des Trigonella piperata, des Corbula striata, des Arca Noe, etc., à un moindre degré pour ces dernières. On verra plus fard quelle est

(1) Pl. 7, fig. 11

(2) Pl. 6, fig. 5 .

(3) Pl. 5, fig. 6 .

(4) Pl. 7, fig. 4.

(5) Pl. 7, fig. 2.

(6) Pl. 5, fig. 3

(7) Pl. 7, fig. 4.

(8) Voyez les planches et les figures des œufs de ces espèces. 
l'origine de cette zone transparente; disons pour le moment qu'elle ne fait pas partie intégrante de l'œuf proprement dit.

L'enveloppe vitelline se remarque difficilement à cause de son peu d'épaisseur ; toutefois on ne peut nier son existence, car, dans quelques cas où l'œuf est rompu ; on voit les granulations du vitellus être retenues en partie par la vésicule germinative, qui vint, entrainée par le courant, boucher l'orifice de la rupture (1). Je ne puis donc partager, en cequi touche les Acéphales au moins, une opinion émise par M. Vogl (2) pour l'Actéon, et qui consiste à considérer le vitellus comme une masse plastique analogue à une boulette de suif, sans membrane enveloppante propre; il arrive souvent de rencontrer l'enveloppe externe plissée autour de l'œuf, qui conserve sa forme parfaitement régulière $(3)$.

Le vitellus ne présente rien de particulier ; il est composé d'une multitude de granulations, auxquelles l'cuf doit sa couleur ; aussi je ne puis m'empêcher de remarquer combien ici le nom de jaune qui lui est quelquefois donné est impropre, de même que la plupart des noms tirés des propriétés physiques; car la coloration qui domine, quand il en existe une franche et bien caractérisée, est le rouge.

Le mélange des œufs à l’eau en détermine fréquemment par endosmose la rupture ; il permet d'apprécier l'état plastique du vitellus, qu'on voit, en effet, s'écouler comme une masse visqueuse, sans que les granulations s'écartent, et c'est à cette plasticité, je crois, qu'est due le plus souvent l'apparence piriforme. Comment pourrait-on expliquer les formes bizarres que prennent les œufs des Gastrochænes, si l'on n'admettait pas une membrane vitelline, et s'il n'existait pas une certaine viscosité qui tient rapprochées les granulations?

La vésicule germinative ou transparente est toujours évidente dans les œufs mùrs, et tombés de l'ovaire ; mais on ne la reconnait que par la compression ou à une sorte d'éclaircie blanchâtre (4), qui

(1) Pl. 7, fig. 11.

(2) Sur l'embryogénie des Mollusques gastéropodes, t. VI (Ann. des sc. nat., $3^{\text {e }}$ série), p. 11, du tirage à part.

(3) Pl. 6, fig. 4.

4) Pl. 7, fig. 2, 4; pl, 6, fig. 5, 4, 3 
tranche en se perdant peu à peu sur la masse opaque gramuleuse du vitellus. Dans l'œuf ovarique, aı contraire, elle apparait avec la plus grande netteté (1). Ses contours bien accusés frappent tout d'abord; aussi n'a-t-elle jamais échappé aux observateurs. Elle parait évidemment formée d'une membrane très mince et transparente, d'un contenu également transparent, dont les granulations sont excessivement légères et peu accusées, si même elles existent.

Quand les œufs sont rompus, la vésicule de Purkinje est presque toujours placée au commencement de la partie allongée. Emportée dans ce point par le courant, elle n'a pas pu franchir l'orifice, elle le bouche.

Quand on soumet une parcelle de l'ovaire à l'examen du microscope, on remarque un grand nombre de vésicules germinatives flottant au milieu des granulations vitellines devenues libres, et animées d'un mouvement brownien très vif quelquefois. Cette apparence a souvent été figuréc surtout par II. Deshayes (2); mais elle ne donne aucune idée de la structure de l'ovaire. Je l'ai moi-même indiquée $(3)$ pour permettre de faire la comparaison avec les figures, qui expliquent réellement la composition de la glande.

La vésicule germinative a, comme les œufs, un volume variable; ainsi, dans le Pectunculus, l'Unio, on la trouve beaucoup plus considérable que dans les autres espèces; il y a aussi des variations tenant à l'état du développement. La forme est toujours parfaitement sphérique.

La tache germinative n'est pas toujours unique, comme e'est le cas le plus fréquent dans les animaux supérieurs; souvent on en voit deux. Dans tous les Acéphales lamellibranches, elle est d'une évidence remarquable ; rarement elle occupe le centre de la vésicule de Purkinje; le plus souvent, elle est rejetée sur un des côtés. Je ne puis eiter d'espèces où il se présente quelques doutes sur son existence ; mais dans les Gastrochènes (4), les Lavignons (5),

(4) Pl. 7, fig. 11 ; pl. 6, fig. 7 .

(2) Loc. cit.

(3) Pl. "i, fig. 7.

(4) Gastrochcena terentina, pl. 5, fig. 5, 6.

(5) Trigonella piperata, pl 6, fig. 7. 
les Mulettes (1), olle paraît plus caractérisée encore que dans les antres.

Dans to us les cas, clle ne m'a jas paru mériter le nom de tache; elle ressemlıle à un corpuscule, ou une petite vessie parfaitement sphéríute, dont les contours, nettement accusés, indiqueraient presifue l'existence d'une enveloppe. Son contenu réfracte la lumière autrement que la vésicule germinative; aussi paraît-elle plus oşaque, et comme remplie de matière un peu huileuse. Dans quelques cas, sur des Nulettes des Peintres, je l'ai trouvée comme finement ondulée, sans arriver cependant jusqu'à un élat chagriné.

Il a paru quelquefois dans son intérieur comme une tache plus petite, également sphérique. Dans la Mulette (2) de la Seine, j’ai toujours trouvé plus d'une tache germinative; le plus ordinairement deux rapprochées l'une de l'autre, l'une est toujours plus petite; dans les cufs peu avancés, quelques granulations les accompagnent. Il ne semble pas qu'il faille, arec de Siebold (3), les regarder comme deux nucléoles accolés l'un à l'autre.

On a dit que l'eau était la cause de l'apparition de la tache germinative ; cette opinion n'est pas soutenable devant les faits qu'on observe dans les Mollusques acéphales; car la constance et les progrès du développement ne permettent pas de douter de son existence. D'ailleurs, en examinant des œufs sans eau, elle parait toujours, seulement elle parait un peu mieux, quand les ceufs sont mélangés à ce liquide.

Ainsi en résumé, l'œuf des Lamellibranches se compose des mèmes parties que dans les autres animaux.

Texture des acini el developpement des oufs.

La texture des acini de l'ovaire a besoin, pour ètre bien vue, d'être éludiée sur les exemples dont l'anatomie est la plus facile. Les Bucardes, les Lavignons, les Vénus, les I utraires, les Pholades, nous offrent ces exemples; mais parmi les Naïdes la Nulette me

(1) Unio pictorum et Unio littoralis, pl. 7, fig. 11.

(2) Unio pictorum, pl. 7, fig. 11.

(3) Anatomic comparéc, Mumel Roret, t. I, p. 282, 1850. 
parait encore plus commode, et c'est d'elle qu'il sera d'abord question. Quelques mots suffiront ensuite pour caractériser les différences ou les ressemblances qui se présentent.

Quand on soumet à l'examen microscopique des acini de l'ovaire de l'Únio pictorum, on remarque qu'extérieurement ils sont limités par une membrane fine, lisse, qui représente un véritable cul-de-sac terminant l'une des dernières ramifications des canaux excréteurs ; qu'intérieurement ils renferment des nufs de grandeur sifférente évidemment sécrétés et produits dans cet endroit, et que pour cette raison on doit les nommer tubes sécréteurs. En multipliant les recherches on ne tarde pas à rencontrer des acini, dont les parois présentent (1) une couche externe de cellules polyédriques remplies de granulations brunâtres fines, ayant presque toujours un noyau ou un petit corpuscule jaune rougeâtre assez apparent. Ces cellules, lont les parois transparentes en dessinent les contours, forment la couche placée immédiatement sousl'enveloppe des acini.

D'un autre côté, en étudiant attentivement les a̛ufs contenus dans l'intérieur, et en les comparant surtout à ceux qui viennent d'être pondus, on remarque une zone transparente très manifeste tout autour d'eux; mais tandis que dans ces derniers la masse vitelline est tout à fait centrale, dans les seconds elle s'approche un peu des bords de la zone $(2)$, qui dans ce point parait prolongée en un petit pédicule. Ce pédicule s’efface dans l'icuf pondı, ou dı moins devient beaucoup plus difficile à reconmaitre.

Si l'on prend des aufs beaucoup plus petits, ayant un diamètre sept a huit fois moindre que les ceufs pondus (3), on voit qu'il n'existe plus de zone transparente, que le pédicule est relativement et absolument parlant plus grand que dans les oufs mùrs. On rencontre souvent des exemples (4) où les hords de ce pédicule re. jetés au dehors indiquent une déchirure et un orifice; il n'est même pas rare de voir la substance interne faire an travers une petite hernie. A ce moment des granulations légères et peu nombreuses

(1) Pl. 7, fig. 5 .

(2) $\mathrm{Pl} 7$, fig. 10

(3) Pl. 7, fig. 9-8

i) I'l. 7, fig. 8 . 
s'étemlent jusqu'au hord de la membrane externe, et entourent une résicule germinative très distincte relativement très grande, af ayant deux taches très nettes quoique petites. Enfin l'on rencontre des états intermédiaires, soit dans la cavité, soit dans la couche des cellules de l'acini.

La zone transparente se développe 1 vers le pòle opposé à celui où est le pédoncule, en formant d'abord un segment qui augmente et se rapproche du point d'insertion dı pédicule; ceci explique comment dans l'œuf, avant la małurité complète et sa séparation de l'ovaire, le vilellus semble rapproché dans un point de la cireonférence.

Dans les cellules de la paroi, on trouve de petites vésicules d'autant plus développées qu'elles sont plus près de la cavité centrale. Ces vésicules en se formant font perdre à la cellule qui les renferme son aspect granuleux ; elles finissent par l'égaler en volume et par la remplir totalement $(2)$.

Enfin si prenant des acini, et les déchirant avec le plus de soin possible, on arrive à une préparation convenable, on voit avec toute évidence que le pédoncule est fixé â la paroi interne, et que l'œuf est comme suspendu dans la cavité.

Voilą les faits, cherchons à les expliquer.

La couche externe de cellules granuleuses (3) compose le parenchyme des acini; dans son intẻrieur se développent les œufs (4) qui se trouvent repoussés en dedans à mesure qu une nouvelle couche parenchymateuse externe se forme. L'œứ, en faisant saillie dans la cavité des acini, reste enveloppé par une membrane qui, en adhérant à la paroi du tube sécréteur, cause le pédicule (5).

En résumé, l'œuf, développé dans une cellule de la circonférence, tombe comme produit de la sécrẻtion dans la cavité de l'organe; ce mécanisme de la production des germes dans l'Unio se

(1) Pl. 7, fig. 10

(2) Pl. 7, fig. 6 .

(3) Pl. 7, fig. 5.

(4) Pl. 7, fig. 6.

(3) La figure 13 de la planche 7 exprime théoriquement la marche de la sécrétion et la composition des acini. 
rapporte done d'une manière remarquable à la théorie des séeréiions, théorie qui tend à se généraliser de plus en plus depuis qu'elle a été mise en lumière par Goodsir.

L'oul' est évidemment enfermé daus une enveloppe qui ne fait pas partie de ses éléments; car il faut considérer la zone transparente qui l'entoure comme une coque; aussi nous parait-il que les auteurs ont fait erreur en disant que, dans certains eas, le jaune se fractionne en dedans de son enveloppe, et tourne dans la membrane vitelline; ils ont pris à tort la capsule détachée de l'ovaire restée autour de l'œuf, pour une partie de celui-ci.

Quant à l'origine de cette eapsule, elle est plus difficile à reconnaitre. L'œuf en grandissant a-t-il été suivi par la cellule qui l'a produit, eelle-ci venant faire saillie dans la cavité des acini, et nr restant attachée au parenchỵme que par un pédicule? ou bien l'œuf, après avoir rompu, par son accroissentent, la cellule où il s'est formé, est-il chassé dans la cavité en poussant devant lui, et en s'en coiffant, une membrane qui tapisse les parois? C'est véritablement ce que je n’oserais déeider. Mais quelle que soit l'explieation que l'on armette, toujours est-il rue les wufs passent des parois des acini dans la cavité avec une très grande rapidité, car on ne trouve fas d’intermédiaire entre ceux déjà très évidents dans les parois et ceux aỹant un pédieule, bien que leur taille soit presque la même.

Ainsi entouré d'une capsule, l'ouf, en grandissant peu à peu, est séparé par un liquide qui vient s'interposer entre lıi et son enveloppe. Ceci ne peut faire un doute, car, dans quelques exemples, le vitellus s'épanche daus la zone transparente entre la membrane. vitelline et la coque (1), et s'échappe un peu par le pédoneule; en sorte que l'apparence de la zone ne serait pas due à l'épaissem. le la membrane, mais bien ì un liquide probablement albumineux et assez épais.

Qu'est-ce qui apparait d'abord, de la tache, de la vésicule ou du vitellus ? M. de Quatrefages pense que dans le Taret 2 e'est la vésienle germinative. Je dois avoner qu'il reste quelques doutes à eet égarl daus mon esprit; car dans des ouls tre's petits, n’étant pas

(1) Pl. 7, fig. 11.

(2) Loc. cit., 1) 202-203. 
eneore enveloppés de leur capsule, la tache paraissait, la vésicule germinative se dessinait vaguement, et les trois éléments semblaient s'accroître en même temps. Quoi qu'il en soit, les germes apparaissent sous la forme d'une vésicule dans l'intérieur des cellules du parenchyme, et il n'est pas rare d'en voir deux ou trois naitre dans la même; quand leur développement commenee, les granulations pâlissent, elles semblent disparaître, ee qui serait loin d'indiquer que les granulations des cellules mères forment plus tard le vitellus comme on pourrait le eroire au premier abord, et comme on l'a cru. Du reste, dans les ceufs très peu avancés, où l'on distingue à la fois la tache, la vésicule et le vitellus, on voit les granulations de ce dernier extrêmement pâles et diffuses prendre peu à peu une teinte plus foncée, et cela surtout autour de la vésienle transparente. A ce moment la membrane vitelline existe, ef les granulations ne peuvent être considérées comme venant du parenchyme de l'ovaire, mais comme se développant sur place dans la eavité même de l'ceuf.

La texture des acini est-elle la mème dans les autres Acéphales que dans les Naïades? C'est ce qu'il nous reste ì examiner. Dans les Bueardes, les Cardites, les Lucines, les Arches, les Trigonelles ou Lavignons, les Peignes bigarrés, lesCames gryphoüdes, les Lutraires, les Pholades, ete., ete., on trouve toujours, fuand on est assez heureux et surtout assez habile pour enlever des lambeaux de glande sanstrop de déchirures, des culs-de-sac parfaitement limités par une enveloppe renfermant en grande (puantité des reufs qui paraissent plus ou moins polyédriques, suivant qu’ils se compriment davantage. Sil'on déchire avee beaucoup de soin ces petits coecums, on voit que les oufs restent suspendus aux débris par des pédoneules eontinus avee leurs capsules. Il parait bien difficile de ne pas voir lì une analogie complète avec eo qui s'observe pour la Mulette. Dans le Cardium rusticum (1), la résistance qu'offrent les pédoneules facilite surtout l'examen. On éprouve plus de difficulté à voir les choses dans l'Arca Noe (2), mais eependant, avec du soin, on arrive ¿̀ préparer quelquefois de véritables grappes d'onufs sus-

(1) Pl. 7, fig. 3 .

(2) Pl, 6, fig. 3 . 
pendus ì un lambeau de substance des acini. Dans la Nacre, la facilité avec laquelle la glande se détruit aux moindres attouchements est extrême, et cependant, dans cet exemple encore, j'ai eu de petits paquets de trois, quatre oufs, réunis par le sommet de leur allongement; aussi n'y a-t-il pas de doute possible quand on considère que toujours les parois des acini sont tapissées par une couche cellulaire (1). Les neufs séerétés dans la paroi du culde-sac vienment ensuite faire saillie dlans sa cavité, jusqu'à ce qu'ils tombent, et deviennent libres par la rupture du pédicule qui les fixait.

Il parait vraisemblable que, dans un grand nombre de eas, les wufs sont entourés par une capsule, et que celle-ci persiste pendant les premières périodes du développement de l'enbryon; aussi ne serait-il pas étonuant que n'ayant pas remouté à la structure de l'ovaire, quelques auteurs aient pris la capsule pour l'enreloppe vitelline elle-même; car, dans quelques cas, la Nacre par exemple, la coque est peu épaisse ; et lorsqu'on n'a pas fait de recherches comparatives, on a de la difficulté, au premier abord, à trouver dans l'wuf de cet Acéphale la même composition que dans celui de la Came gryphoïde par exemple.

Les auteur's se sont peu occupés de la structure de l'ovaire. II. de Quatrefages a fait comnaitre celle de la glande du Taret. II. Deshayes a donné un grand nombre de dessins à l'appui de ses idées sur le mode de développement des germes. Il n'est guère possible de se faire une idée bien nette de l'opinion de ce dernier auteur, car elle est revêtue d'expressions qu'on est peu habitué à voir réunies. Sa théorie d'une attraction partieulière des granulations autour d'une vésicule n'est pas conforme à la vérité; car Jorsque l'oruf est déjà formé, le vitellus est encore transparent, et e'esi dans l'intérieur de sa membrane que se développent suecessivement les granulations vitellines. D'ailleurs quels sont les exemples daus lesquels l'ouf est ouvert? Je citerai les passages les phus saillants (qui paraissent résumer les opinions de M. Deshayes, laissant le lecten juge; on y remarquera que la disposition du 
pédoncule a été aperçue, mais que l’interprélation est difficile à admettre, car elle semble étrange et en dehors de tout ce qui est connu.

Dans des cellules, l'auteur voit « chaque embryon renfermé dans " une enveloppe ovalaire, terminé par un bec ouvert, plongé dans " le parenchyme de l'ovaire. Les embryons sont composés d'une " matière jaunâtre, granuleuse, qui, vue par transparence au mi„ croscope, prend une couleur grisâtre. Une petite vésicule trans" parente, vésicule de Purkinje, existe vers le centre de tous les „ embryons (1). " Voilà pour la structure de l'ovaire. Quant au développement de l'neuf (2), il est ainsi décrit aux conclusions qui terminent la monographie des Lavignons (3) : «L'embryon se déve" loppe dans l'ovaire de la même manière que dans les autres Mollus» ques de la même classe. Une vésicule vivante, attachée sur les pa» rois internes, attire à elle des globules blanchâtres contenus dans la " masse de l'ovaire; à mesure que le nombre de ces globules s'ac» croît, elle se développe sous la forme d'une ampoule, que l'on voit " se modifier successivement jusqu'au moment où elle a acquis " celle d'une fiole à long col. Ce col, fixé dans la masse ovarienne, » reste ouvert, et c'est par lui que de nouveaux granules s'intro» duisent et s'ajoutent i ceux qui entourent déjà la vésicule. Lorsque „ l'embryon est parvenu au terme de son développement, l'extré"mité du cou se ferme, se détache, et il devient libre dans la coque » de l'œuf. En même temps la coque s'est développée, s'est remplie » d'un liquide transparent, et l'extrémité de l'embryon reste encore » ouverte à travers les parois de l'œuf jusqu'au moment où celıi-ci » est enfin ciétaché des parois de l'ovaire. »

Les conduits excréteurs ont une texture bien simple; ils sont formés d'unc couche externe, peu épaisse, transparente, dans laquelle des trainées et des granulations vagues se distinguent ęà et lì indiquant comme une disposition un peu fibreuse; peut-être y a-t-il même quelques fibres musculaires; à l'intérieur on trouve

(1) Mollusques de l'Algèrie, p. 226.

(2) On doit supposer que les mots oufs et embryons sont synonymes pour l'auteur.

(3) Loc. cil , ए. 506 . 
une couche de cellules formant un épithélium (1) très remar(uable it chaque cellule, excessivement transparente, se dessine firiblement, elle a un noyau bien marqué, et est couverte sur toute sa surface libre par des eils vibratiles d'une grande longueur et d'une vivacité très grande. Cette structure cesse là où commence le parenchyme du cul-de-sac sécréteur. La direction du mouvement de l'épithélium est de dedans en dehors; elle explique la sortie des oufs, la ponte.

Le rôle physiologique de cet épithélium est beaucoup plus important qu'on ne saurait le croire au premier abord. Certainement les contractions musculaires de la paroi abdominale pourraient expliquer la sortie des œufs par les pressions qu'elles exercent sur. tout l'ovaire; mais dans les Moules, où la glande est placée dans les parties les moins musculaires du manteau, les mouvements vibriltiles des conduits exeréteurs peuvent à eux seuls expliquer la ponk. J'ai vu dans une Mulette, dont j'avais ouvert sous l'eau l'ovaire et les principaux trones excréteurs, tous les œufs épars a la surface de la déchirure être attirés par les courants, venir former des trainées, et marquer ainsi la place des conduits. La ponte continuait à se fuire à ciel ouvert, si je puis ainsi dire, et quoique les oeufs ne fussent plus soumis à l'influence des pressions intérieures.

J'ai constaté ce fait sur les Moules, les Arehes, les Dattiles, les Pinnes marines, les Bucardes, ete., toujours avec une grande facilité; on peut même sur la Moule, au monnent de la ponte, quand on a détaché une des valves de la corquille, voir dans les canaux excréteurs un cylindre central formé par les oufs, tenus éloignés rles parois par les mouvements ciliaires; on le voit même quelquefois se mouvoir.

En résumé, quand l'œuf se détache de la paroi du tube sécréteur, enveloppé on non par une capsule, il tombe dans la cavité poussé par ceux qui le suivent, il arrive au canal exeréteur, où les cils vibritiles s'emparent de lui et le transportent au dehors. On pourrait peutêtre considérer les lamelles dont parle $\mathbf{1 1}$. de Quatrefages (1), et qui remplissent l'ovaire du Taret après la ponte, comme les débris des ('apsules des coufs.

1) Pl. 7, fig. 12, épithélium vibratile des conduits de l'ovaire del'Unio pictorum.

12) Loc. cit, 1. 205 . 
$\S$ II.

\section{Organes males (testicules).}

Eulre le testicule et l'ovaire d'un Acéphale, la différence se réduit presque aux produits de la sécrétion; anssi n'aurons-nous que peu de détails à domner sur la position, la forme et les autres earactères généraux de la glande mâle : ce qui a été dit pour les femelles pourrait être, en grande partie, reproduit ici.

Le testicule occupe l'abdomen et les còtés du foie; il contracte les mêmes rapports que l'ovaire : cola est si vrai que les auteurs qui admettentl'hermaphrodisme les ont constamment pris l'un pour l'autre.

Lin seul caractère est très différent, c’est la couleur. On a pu remarquer que dans l'ovaire des teintes rouges, souvent des plus belles et des plus riches, s'offraient ì l'olservateur; jamais le testicule ne s'est présenté dans de scmblables conditions. Je ne prétends cependant pas (que l'organe mâle ne puisse avoir une teinte plus ou moins rougeâtre; mais la couleur la plus habituelle a été ou le blane mat parfois nacré, ou le blane jaunàtre quelquefois lavé de bistre. La première teinte se remarque surtout dans les Clovisses (1), les Prìres (2), les Gastrochènes, la Donace (3), etc. ; et la seconde dans les Bucardes, surtout dans les Huitres vermeilles (4). Dans les Arehes, les Dattiles (5), le jaune est un peu ferrugineux. Cette différence de la couleur cutre le mâle et la femelle, quand on s'est éclairé d’ahord de recherches microscopiques, devient précieuse par la recomnaissance des sexes: elle ne trompe presfue jamais.

\section{Structure.}

Ici encore les détails vont être abrégés par les développements consacrés à l'étude de l'ovaire; la structure de l'or-

(1) Venus decussata.

(2) Corbuba striata.

(3) Donax anatinum.

(4) Spondylus grederopus

(5) Modiola lithophaga. 
gane mâle ressemble beaucoup à celle de l'organe fenelle, ì ce point que dans le Peigne bigarré, l'Huître vermeille, la couleur laissée de côté, l'œeil ne peut discerner aucune différence, et l'intervention du microscope est tout à fait nécessaire. Ceci explique les erreurs des naturalistes rui, partant d'une idée préconçe, n’ont pas employé cet instrument, ou l'ont employé sans prendre de précautions suffisantes.

Toutefois, après de nombreuses recherches, il m'a paru qu'on pouvait apprécier dans quelques espèces des différences légères ; que les acini de l'ovaire n'étaient jamais aussi allongés que ceux du testicule; que lorsque les dernières granulations de ce dernier se rémissaient, elles formaient des grappes plus serrées. Mais, je le répète, si l'on peut, après une étude spéciale de ces organes, arriver à recomnaitre leur différence, il est des cas intermédiaires où la distinction devient impossible.

Ainsi nous admettons dans le testicule, comme dans l'ovaire, des acini, ou dernières ramifications appréciables de la glande, des lobules primitifs, secondaires, des lobes, en n'attachant à ces mots qu'une importance tout à fait secondaire.

La forme et le mode de groupement des acini permettent de rapporter à deux types principaux toutes les différences.

Dans le premier, on voii les culs-de-sac, très nombreux, fortement renflés, suspendus à un rameau des tubes excréteurs par IIn pédoncule, une sorte d'étranglement. Il en résulte une apparence de grappe parfaite (1), que les Bucardes, surtout le Rustique, présentent d'une manic̀re remarquable; il est f.cile, quand on s'est livré longtemps à res sortes de recherches, d'enlever avec le tube excréteur toute la grappe, qui se compose pent-être d'une vingtaine d'acini; on peut observer cela sans préparation, mais avec moins de netteté dans le Peigne varié, sur les lobes hépatiques, ou bien dans l'Huître vermeille sur l'anse intestinale qui, placée dans l'abdomen à l'extrémité de la partie en bosse de polichinelle (2), se rapproche de la surface, et cuui, dilatée par des liquides transparents,

(1) Pl. 9, fig. 1 .

(2) Pl. 9, lig. 13 . 
n'est quelquefois separée de l'enveloppe extérieure que par une eouche mince de lobules primitifs.

Le second type se remarque, avee la plus grande facilité, dans les Clovisses (1), qui abondent, on peut le dire, dans tous nos ports de mer; les acini ne sont plus globuleux, ils sont cylindroïdes, et s'insèrent de distance en distance sur le tube excréteur; en sorte que le lobule a la forme et l'apparence de ces arborisations, de ces dendroïdes que l'on rencontre sur les lames de certaines roches.

Entre cette forme et la première il y a une différence très grande, qui, cependant, n’a au fond qu'une importanee peu marquée, et qui tient ì un allongement de la partie du tube exeréteur dans le point où s'insèrent les euls-de-sae producteurs du fluide séminal. Cette eirconstance les éloigne et les isole ; ici le lobule primitif est loin d'être aussi distinct et earactérisć que dans le premier cas, ee qui montre bien que l'importance attachée à ces expressions ne doit avoir rien d'absolu.

Entre ces dispositions extrêmes on trouve des intermédiaires, et, dans beaucoup de cas, le rapprochement des éléments, les nombreuses brides museulaires empêchent, comme pour l'ovaire, d'arriver aussi facilement à se rendre un eompte très exact de ce qui existe. Ylais ceci tient à une difliculte anatomique, et ne peut infirmer en rien les résultats.

Dans les Corbules, les Mactres, les Myes, les Lutraires, les lobules paraissent bien plus rapprochés, par la forme, de ceux des Bucardes que de ceux des Clovisses; cependant, dans le premier exemple, on roit un passage de l'un i l'autre. On distingue bien les lobules primitifs dans la Pholade ( 2 sur le dos, où la couleur, d'un blanc laiteux, les rend très évidents sur le fond brun-vert du foie.

Quant aux conduits excréteurs nés de culs-de-sac glandulaires, ils se rémissent en se rapprochant de l'orifice, et forment des ea-

(1) Venus decussata, pl. 9, fig. 4.

(2) Pholas daclylis de la Rochelle. Il faut toutefois remarquer que si l'on observe au moment de la plus grande activité des glandes le corps tout entier paraît blanc, et les canalicules du testicule se dessinent avec moins de nettelé. 
naux de plus en plus volumineux, qui, de même que dans l'ovaire, se terminent en un seul tronc, et s'ouvrent dans un joint du corps tout semblable. La position de l'ouverture des deux glandes est done identique dans les deux sexes d'une même espèce; aussi ce qui s'applique à l'un peut s'appliquer ì l'autre.

Quant aux lobes principaux, on peut, comme pour l'ovaire, les distinguer en sus-hépatiques et abdominaux; en général, deux branches principales des conduits excréteurs se rendent à chacun d'eux, souvent une branche moyeune vient de la partie située entre les deux lobes principaux, et s'unit au canal abdominal, dans le point où celıi-ci se joint à celui qui vient du lobe sus-hépatique. Cela s'observe également dans l'ovaire.

En résumé l'applareil mâle se compose de deux moitiés symétriques, le testicule gauche est distinct du testicule droit, mais il se mêle avee lui sur ladigne médiane; et pas plus que l'ovaire, je ne puis nier ou affirmer yu'il y a ou qu'il n'y a pas de communication entre les deux glandes.

En terminant, je ferai observer que pour les mâles comme pour les femelles, dans l'Anomie et la Moule, la position du testicule est différente, que la glande est placée dans l'épaisseur du manteaur.

\section{Texlure.}

D'après ee qui précède, on peut voir que l'histoire du testicule ne commence ì proprement parler quici. Nous suivrous la même marche que dans la description de l'ovaire. Nous étudierons d'abord le produit sécrété et le Spermatozö̈de, puis l'acini, en cherchant ì voir quels sont les rapports des premiers avee les seconds; en d'autres termes comment se développent les filaments spermatiques, comment sont composés les canaux excréteurs; telles sont les questions que nous allous aborder.

\section{Spermalozoïde.}

Les mèmes moyeus yui ont servi ì se procurer l'ouf peuvent servir aussi à avoir les spermatozoürles; les piquires, les déchirures

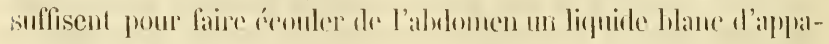

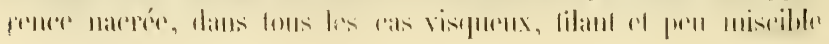


l'abond a l'aun : c'est le sperme daus lequel fourmillent les filaments spermatiques.

Que l'on prenne du sperme dans le fond de la glande, ou que, frar une éjaculation forcée, on l'obtieune à la sorlie des orifices, les caractères des filaments sont ì peu près les mêmes, ì moins qu’ils ne soieut pris dans des glandes peu développées.

Tontes les formes se réduisent à deux tỵes différents, bien distincts. Tantôt la tête est allongée ; tantôt, au contraire, clle est globuleuse : la queue, ne variant que pour la longueur, n’imprime pas de earactère spécial.

La Lime (1), le Gastrochène, l'Anomie, la Chame, le Lithodome, le Peigne bigarré (2), le Spondyle, et l'un des Bucardes $\mathbf{3}$, la Moule (4), l’Unio (5), les IIyes, les Lutraires, les Pétricoles (6), les Pholades, les Arches, les Solens, les Donaces 7), etc., présentent la forme plus ou moins globuleuse; tandis que les Bucarles (8), les Cardites $(9$, les Lucines, les Corbules (10), les Vénus (11), les Pétoneles, les Laviggnons (12) présentent la forme allongée.

Il ne faut pas attacher à ces formes trop d'importance, puisque dans un même genre des espèces offrent les deux types; cepenlant, à part cette exception, il faut remarquer qu'elles sont en rapport avec les relations naturelles des genres.

Nous devons signaler de légères variétés, que nous verrons, dans quelques cas, faire le passage entre les formes les plus extrêmes.

Quand la tête est allongée, tantôt elle est droite, comme dans le

(1) Pl. 9, fig. 14.

(2) Pl. 2, fig. 8.

(3) Cardium ciliare

(4) Pl. 6, fig. 2'.

(5) Pl. 7, fig. 14.

(6) Pl. 9, fig. 15.

(7) Donax anatinum.

(8) Pl. 9, fig. 3.

(9) Pl. 9, fig. 12.

(10) Pl. 9, fig. 11.

(11) Pl. 9, fig. 7 et 8

(12) Pl. 7, fig. 15. 
Pectunculus pilosus, la Venus decussata, les Cardiums, la Trigonella piperata; tantôt courbée en forme de faucille, conme dans la Corbula striata; tantôt enfin, sans être courbe, elle est tordue sur elle-même et ressemble à un Spirillum, ainsi que cela s'observe dans le Cardium rusticum (1). Voilà daus un même genre une nouvelle différence qui montre encore le peu d'importance qu'il faut attacher à la forme. Quant à la disposition cque l'on rencontre dans la Corbula, on pourrait peut - ètre la rapporter au développement; je dois dire eependant qu'elle m'a paru constante, quoique peu marquée.

Le plus souvent la tête ou le corps se termine en pointe en avant, mais elle est effilée en arrière dans les Lavignons (2); dans les Cardium, surtout lo rusticum, elle est très obtuse.

Les variétés de forme du type où la tête est globuleuse sont plus nombreuses et moins marquées encore. Elles portent surtout sur la terminaison antérieure et moins sur la postérieure. Ainsi, dans la Petricola striata (3), l'Anomia ephippium, la Chama griphoüdes, la Modiola lithophaga, la tête est conique, et son sommet fournit l'insertion à la queue. Dans le Jytilus edulis (4, la Donax anatinum, au contraire, e'est l'extrémité opposée à l'insertion caudale qui est allongée et pointue; dans la Moule la pointe est excessivement aiguë et marquée, ce rui donne an Spermatozö̈de l'apparence piriforme. En général, quand le filament réfracte peu la lumière, et qu`il échappe facilement aux recherches, on est tenté de prendre son extrémité effilée pour le commencement de la queue; mais ce n'est pas toujours à cette partie que celle-ci s'insère. Dans une Arca Noe, j'ai une fois reneontré des filaments dont la queue s'attachait, tantòt sur l'extrémité renflée, tantôt sur l'extrémité effilée du corps, ce qui prouve une fois de plus que la forme est bien secondaire. J'ai répété cette observation, dans mon dernier voyage sur les côtes de Bretagne, pour d'autres espèces.

Entre ces formes globuleuses, tronquées, ou effilées en avant,

(1) Pl. 9, fig. 3.

(2) Pl. 7, fig. 15.

(3) Pl. 9, fig. 45.

(4) Pl. 6, fig. 92 
et ces formes allongées cylindrö̈ques, également obtuses ou pointues, viennent se placer eclles que l'on observe dans la Mulette d'eau douce (1) déjà indiqnée depuis 1825 par Prévost de Genève. Les spermatozoüdes de ces animaux ressemblent un peu à un biscuit à la cuiller. Ils ont leur corps renflé aux deux extrémités, ce qui les fait paraitre comme étranglés vers le milieu.

La grandeur des filaments spermatiques des Lamellibranclies n'est pas moins variable que leur forme; ici comme pour l'ouf on ne rencontre aucun rapport entre la taille de l'animal et celle du spermatozoïde. Ainsi dans la Rupicola striata, la tête est plus considérable que dans le Pecten varius. Cependant ces individus ont des proportions bien différentes. Il en est de même dı Spondylus goederopus, dont le filament tout entier est plus petit que dans le Pecten varius. De tous les spermatozoïdes à forme globuleuse, ceux des Mytilus edulis et des Unio pictorum et $\boldsymbol{U}$. littoralis sont les plus grands.

Considérés en général, les tilaments à corps allongé sont ceux qui offrent la plus grande taille ; entre eux ils varient aussi, et leurs proportions ne sont pas plus en rapport avec la taille des espèces auxquelles ils appartiennent que les autres. Ainsi à part les Lavignons, dont la taille moyenne se rapproche de celle des Corbules et des Bucardes, etc., et dont les animaleules sont véritablement les géants de la classe (2), la Gastrochona tarentina a présenté les filaments les plus gros, bien que sa taille soit la plus petite de toutes les espèces étudiées.

La longueur de la queue est plus ou moins grande, et sa transparence, dans quelques cas, la ferait souvent échapper à l'observationsi l'on n'employait des moyens d'éclairage propres à la faire découvrir.

Les spermatozoïdes ont les mouvements d'autant plus marqués et plus vifs qu'on les observe plus loin du parenchyme glandulaire et mélangés à de l'eau; quand on les prend dans la partie profonde de la glande, ils sont rapprochés et souvent unis, ce qui les rend

(1) Unio littoralis, U. striata, U. pictorum, pl. 7, fig. 14.

(2) Cette taille considérable des spermatozoïdes de la Trigonella piperata en facilite beaucoup l'étude, et M. Deshayes doit avoir employé des grossissements bien insuffisants pour ne les avoir pas recounus. 
presque immobiles. C'est tout au plus alors si leurs quenes ondulent légèrement.

L'eau parait avoir sur eux une influence hemreuse bien différente en cela de celle qu'elle a sur ceux des animaux supérieurs; mais l'eau douce détruit la vitalité des filaments des acéphales marins, de même que l'eau salée fait disparaitte celle des filamentsdes $U$ nio. Les conditions normales de la féeondation expliquent ces faits.

Les mouvements sont de deux sortes. Les uns appartiennent i la queue : ils sont ondulatoires comme dans les animaux supérieurs; les autres se passent dans le point d'union du corps et de l'appendice caudiforme. Ceux-ci ressemblent ì des flexions brusques de la première partie sur la seconde. En s'ajoutant, ces deux mouvements font progresser le filament avec une telle rapidité qu'il n'est pas rare d'en voir quelques-uns traverser le champ du microscope comme un trait, on a même peine à les suivre.

On trouve, dans les auteurs, peu de chose sur les filaments spermatiques des Acéphales lamellibranches. Siehold (1), dans un long article sur les spermatozoïles en général, n’a donnẻ de figures que pour trois espèees; quant i MM. Wagner et Leuckardt (2), ils se sont contentés de dire que les filaments étaient eylindriques dans les Unio, Cyclas, Clavagella, ou bien piriformes dans les Mytilus et Pholas. J'ai eité la figure exacte qu'a donnée Prévost (3). Enfin II. de Quatrefages (4) a, dans son travail sur le Taret, douné un dessiu qui se rapproche beaucoup de ce qui existe dans la Moule. Mais ancun auteur n'aỵant fait un travail d'ensemble sur le sujet n’a insisté d'une manière générale et comparative sur les variétés de forme et de grandeur.

Texlure des acini ou tube sécréteur et développement des spermalozoïdes.

Il y a, dans la texture du canal séeréteur des Acéphales lamellibranches dioïques une telle similitude pour toutes les

(1) Voyez Siebold, Arch. de Miuller, 1837.

(2) Wagner et Leuckardt, article SEmen, dans le Cyclopedia of analomy and physiology de Tood.

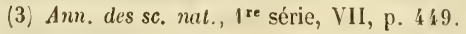

(4) Ann. des sc. nat., 3 série, $18: 9$. 
espèces, que ce qui sera dit pour une s'appliquera, d'une manière presque absolue, aux autres. Nous reprendrons done les deux exemples qui nous ont servi de type pour en indiquer la disposition générale, le Cardium rusticum el la Venus decussata.

Dans l'un et l'autre cas, en soumettant ì un faible grossissement (60 diamètres), l'un des culs-de-sac terminaux des condıils excréteurs, on voit que les bords sont transparents, tandis que le centre est obscur ; si l'on augmente le pouvoir grossissant (180 diamètres à 230 diamètres), on ne tarde pas à s'apercevoir que la partie transparente correspond au parenchyme cellulaire des acini, tandis que la partie obscure est due à l'accumulation dans le centro du conduit des parties sécrétées, aux spermatozoïles soit réunis en paquets, soit déjà lihres.

Dans le parenchyme des acini, avee un grossissement considérable (500 à 700 diamètres), on trouve, sans distinction d'espèce, seulement avec des différences de volume, des éléments toujours semblables. Ce sont de petits corpuscules réfractant assez vivement la lumière, et offrant un aspect constant qui les fait reconnaître et les rend caractéristiques. Ces corpuscules forment une couche assez épaisse tout le tour du cul-de-sac, ce qui explique l'apparence indiquée plus haut.

Leur contenu est finement granuleux, ct, dans quelques cas, on y trouve un noyau ordinairement peu considérable et pen distinct.

Les différences que l'on remarque dans les espèces se rapportent exclusivement an volume, elles coïncident toujours avec les variations de taille des spermatozoïdes. Ainsi dans la Gastrochcena tarentina, filament spermatique très grand, cellule du parenchyme relativement considérable, de même dans l'Unio pictorum, surtout dans le littoralis, la Trigonella piperata, cte., la différence de grandeur peut aller plus loin que du simple au double (1).

La forme est polyédrique par suite de la compression réciroque.

La couche représente à elle seule toute la partic productrice du sperme, elle est enfermée et entourée extérieurement par la membrane mince qui enveloppe les acini. Leur multiplicatiou

(1) Voyez fig. 14 et $15, \mathrm{pl} .7$. 
semble se faire dans l'intérieur d'autres corpuscules plus grands; cependant il faut dire que rarement on rencontre ces exemples montrant le développement endogène. La Gastrochcena tarentina, l'Unio littoralis, l'A nomia ephippium, sont les exemples qui ont le mieux favorisé l'ohservation. On pourrait peut-être s'expliquer cette difficulté à observer ce mode de développement en admeltant que la cellule mère se détruit promptement et avec beaucoup de facilité. Toutefois, d’après les auteurs qui ont éerit à ce sujet, il serait loin d'en c̀tre ainsi (1). Ce qu'il m'a toujours été impossible de bien éclaircir, e'est de savoir si les corpuscules ou cellules secondaires inclus se sont développés séparément et ont grandi peu à peu jusqu'ì ee qu’ils aient rempli la cavité de la cellule mère ; ou bien si celle-ri s'est partagée en un plus ou moins grand nombre de vésicules secondaires, par la production de cloisons adventices, comme cela a été déerit. Je croirais plus volontiers à cette dernière opinion, ear dans quelques cas, les cellules internes étaient ¿ peine appréciables, et cependant la cellule mère ressemblait, 'fu'on me passe la comparaisou, à un ouf qui commence à se fraclionner; des lignes à peine sensibles indiquaient que plus tard il y aurait des rellules bien développées, mais qu'elles n'avaient eneore que leurs limites de masquées. D'un autre côté, jamais je n'ai même cru voir de petites cellules en voie de développement dans la grande cavité; je dois eneore ajouter que les noyaux regardés romme centre de développement des cellules étaient loin d'être évidents dans tous ces cas.

Quoi qu’il en soit, il paraît hors de doute que les éléments cellılaires du parenehyme des acini, se mulliplient sur place dans la paroi du tube sécréteur par la voie endogène $(2$.

Oì et comment sont produits les filaments spermatiques?

Quand on étudie les spermatozö̈des dans les différentes classes d'animaux, on remarque bientôt qu'ils sont fréufuemment réunis en paruets, dont la disposition très variable est quelquefois remar-

(1) Siebold, Miiller's Archiv, 1837. - Wagner and Leuckart, Cyclopedia of anatomy and physiology, article SExEx. - Kölliker, Développemenl des Spermalozoüdes dans l'Ileli.x pormatia.

2) Vovez fig. 11, 1:, pl. 7; fig. 9. 11, 12, pl. 9 
quable; on s'est batsé sur celle rémion pour explifuer le développement et l'on a, d'un autre còté, invoqué le développement pour se rendre comple de la formation des paquets; il y a lì un un ecrele vicieux. Dans les Acéphales, les filaments sont groupés d’une manière plus ou moins irrégulière; janrais je n’ai rencontré les paruets enfermés dans une cellule. Cependant mon attention était éveillée à cet égard, et mes observations ont été trop multiplices pour n'avoir pas rencontré quelques exemples. C'est toujours par la tête qu'on trouve les spermatozoïdes rapprochés et unis; les queues rayomment dans tous les sens; quand la tête est allongée, l'aspect change un peu mais sans offrir rien de différent, si cen'est toutefois dans la $V$ enus decussata $(1)$, où les paquets très gros forment de véritables masses 'ylindriques arrondies à une extrémité. Ces masses sont formées par les têtes des filaments qui ne rayonnent plus autour d'un centre, mais bien tout le long d'un axe. C'est à ces masses qu'est dù l'aspect particulier du centre du tube sécréteur. Ces cỵlindres sont formés d'une quantilé prodigieuse de filaments et aequièrent quelquefois une assez grande longueur.

Quand on détache des lambeaux des acini, on voit souvent (2) d'un côté des cellules de la couche parenchỵmateuse, et de l'autre des spermatozoïles dont la tête adhère, tandis que la queue est libre. Cette chose, qu'on observe dans tous les eas, n'a pas échappé ì M. de Quatrefages qui en a donné un dessin dans son histoire du Taret. La première idée que fait naitre ce fait, c'est que les filaments se développent dans les corpuscules du parenchỵme, et cela ne me parait pas douteux; chaque cellule ou corpuscule, comme on voudra l'appeler, produit un filament; la paroi, en se détruisant, laisse tomber son produit dans la eavité du tube, mais il n'y devient pas libre tout d'un coup ; sa tête reste adhérente, pendant que sa queue ondule et se débarrasse la première. C'est surtout dans les espèces dont les filaments sont allongés que l'on voit cette origine. Dans la Venus decussata, la C'ardita sulcata (3) et le Pectunculus pilosus, j’ai rencontró des filaments dont la tête courbée pré-

(1) Pl. 9, fig. 6-7.

(2) Pl. 9, fig. $14\langle$ a $\rangle$ 12.

(3) Pl. 9, fig $12(r)$ 
sentait, dans sa courbure, une apparence de cellule rappelant le corpuscule du parenchyme; dans ces mêmes exemples, j’ai vu des corpuscules tout ì fait analogues ì ceux de la paroi, renfermant, sur un de leurs côtés, un corps allongé qui ressemblait à celui d'un spermatozö̈de (1). Dans la Chama griphoüdes, où la tête est différente, je l'ai aperẹte très évidemment près de la paroi, et même il s'est présenté des eas où la cellule semblait avoir un prolongement caudiforme.

Mais je dois dire que, malgré tous mes efforts, je n'ai jamais ju distinguer la queue dans le corpuscule. Cela se comprend quand on songe que mème sur des spermatozoüdes libres, il est des espèces où on l'observe difficilement.

En résumé, il me parait probable qu'ici la théorie générale des sécrétions peul expliquer la formation du sperme. Nous voyons, en effet, dans un enl-rle-sac, une couche ecllulaire produite par la multiplication endogène, dont chaque élément renferme un spermatozö̈de qui devient libre par la disparition de la cellule produetrice; cette couche, se développant à la surface, repousse et rejette en dedans du tube séeréteur les produils les plus anciens qui tombent ainsi dans les canaux excréteurs.

Là s'arrête ce que nous avons vu ; quant à dire si le noyau de la cellule a formé le corps du filament, et les granulations la queue, en s'ajoutant à la suite les uns des autres (2), ou bien si la cellule tout entière s'est allongée, e'est ce qu’il ne m'a pas été donné de roir. Aussi ne suivrai-je pas MM. Wagner et Leuckardt (3) d'une part, et M. Kölliker (4), de l'autre dans leurs appréciations sur l'origine des filaments.

Le désir de faire tout rentrer dans la théorie cellulaire peut expliquer des iclées qui, bien que soutenues par les plus habiles micrographes, n'en paraissent pas moins devoir être démontrées avec plus d'évidence.

(1) Pl. 9, fig. $12(d)$.

(2) Cyclopedia of anatomy and physiology de Tood, article Semex.

(3) $I d .$, Wagner and Leuckart, p. 499.

(i) Développement des spermatozö̈des de l'Helix pomalia ( 1 ew Denlischrift de Allgem, VIII, 18 i6, p. 4, pl I, fig. 1 ì 10). 
Faut-il, avec ces auteurs, considérer le groupement des spermatozoüdes comme la conséquence de leur réunion, dans une cellule mère des corpuscules ou cellules secondaires qui les ont produits? Je ne le erois pas pour les leéphhales, car jamais, comme dans l'II lix, je n'ai trouvé de paquet spermatique dans une grande cellule. D'ailleurs, comment admettre que les longs eylindres de la $V$ enus se soient produits dans une seule cellule mère. Le nombre. des corpuseules secondaires qu’ils représentent est considérable et nous conduirait à admettre l'existence de vésieules mères vraimen! monstrueuses.

I)'ailleurs on n'a pas besoin de supposer leur séjour dans la cellule mère, pour s'expliquer leur réunion. Je dirai même que l'on ne comprend pas comment cette condition ferait nécessairement rassembler, d'un même côté, toutes les tètes qui se fixeraient après avoir été éloignées et séparées, tandis que l'on voit très bien que, dans une masse cellulaire dont charue cellule est adhérenle à la voisine, la destruction progressive des utricules, permeltant d'abord aux queues de flotter, tient les eorps unis de moins en moins solidement, à mesure que le travail de la séerétion les rejette plus en dedans. Dans une autre division des Lamellibranches, dans les Huìtres, on verra cela encore plus évidenment.

\section{Conduits excréteurs.}

Nous aurons à dire ici peu de chose qui ne se rapporte à ee qui a été indiqué pour les femelles. La même texture s'observe, la couche parenchymateuse, caractéristique des culs-de-sac sécréteurs cesse dans ces canaux, elle est remplacée par un Epithelium vibratile des plus vifs et des plus caractérisés. Son but est le même, il sert à l'éjaculation qui peut avoir lieu sans que les contractions soient absolument nécessaires.

Ici se termine ce que nous avions ì dire sur les Acéphales lamellibranches diö̈ques. Il reste établi et mis hors de doute que, dans toute cette division, les sexes sont parfaitement distinets el caractérisés, qu’ils sont portés par des individus différents et que le nom général rui leur est donné se troure par cela même justifié. 
II.

Acéphales monoïques.

PREMIÉRE DIVISION.

Aceiphales monsïques à glandes distinctes.

Jusqu iei les sexes nettement séparés, sans que le plus léger doute fùt jossible, nous ont permis de refuser aux individus d'un grand nombre d'espèces la faculté de se féconder eux-mêmes, mais il est d'autres cas où il n'est plus possible de nier l'existence de l'hermaphrodisme. Il reste done à s'oceuper des Acéphales portant a la fois les deux sexes. Le nombre en est très restreint. Quatre genres seulement, les Pandores, les Bucardes, les Pectens et les Huîtres, se sont présentés dans ces conditions. Toutefois Siebold (1 et Krohn (2) ont aussi considéré les Cyclas et les Clavagelles comme hermaphrodites; on se rapjelle quelle distinction a été établie en commençant entre les Lamellibranches nonoïques, suivant que les glandes sexuelles sont séparées ou confondues.

A la première division appartiennent les Pectens ef les Pandores. Les especes mont paru, sur des points très éloignés, à des noments différents, loujours dans les mêmes conditions, ì Mahon, ì IIarseille, à Celte, à la Rochelle, à Saint-Jacut-la-Mer, Loujours l'hermaphrodisme s'est offert si nettement qu'il ne pouvait être douteux.

Le Lamellibranche monoïque à glandes distinetes fail le passage entre la première et la seconde division; la séparation des glandes est tellement nette que chacune des portions de l'abdomen des Pecten Jacobeus, P. maximus, P. glaber, Pandora rostrata, représente un individı tout entier des Lamellibranches diö̈ques. Cette nettetí daus la distinetion, nous permettra d'abréger beaucoup les détails, car pour chacune des portions mâles on femelles il n'y aurait yu’ì répéter ec qui a été dit, relatirement à la première division.

Dans les premières espoiees qui viennent d'ìtre citées, l'abdomen contourné en-dessous du muscle des valves, semblable ì la bosse de Polichinclle, est, daus loute sa partic postéricure, coloré en ver-

(1) Müller's Archiv, 1837, p. 383.

(2) Kroln, Frorieps neue nol, $n^{\circ} 3: 56$, p. 32 . 
millon, quelquefois d'une teinte très riche 1, mais dans quelques cas on pourrait ètre induit en erreur, car un pigment noirâtre la voile et la dérobe aux regards. Pour la faire reparaitre, il suffit de hrosser l'animal arec un pinceau.

Ce qui frappe d'abord, e'est la netleté de la ligne de séparation entre la partic rouge et la partie blanche : celle-ci, antéricure, correspond au testicule; celle-là, postérieure, à l'ovaire; les doutes sur la nature des glandes et leur distinction ne peuvent se présenter à l'esprit. Cette disposition avait été indiquée, du reste, déjì depuis longtemps par II. Nilne Edwards (2.

La texture de chacune de ces glandes ressemble à celle qui a été décrite avec soin en commençant. L'ouf ne présente rien de particulier; e'est au vitellus qu'il doit sa couleur, et l'ovaire est formé d'acini dont la structure ne diffère pas de celle d'une Huître vermeille par exemple, et dont la réunion produit des lobules primitifs secondaires, ete., absolument comme dans l'exemple arec lequel je fais la comparaison.

Les spermatozö̈les (3) ont une tète globuleuse fréquemment conique en avant, une queue assez longue que l'on distingue facilement; ils se développent et se meuvent comme il a été dit pour les Lamelibranches diö̈ques.

Les conduits excréteurs méritent de nous oecuper un instant, non qu'ils présentent quelque chose de particulier, mais bien frar ce qu'ils ont été méconnus. Les deux glandes s'arrêtent au-rlessous du foie, et rie remontent pas sur ses côtes, non plus que vers le dos; différence très marquée avec ce qui s'observe dans le P'ecten varius, où nous avons cherché sur le foie à débrouiller la structure de l'organe. Les canaux (4) forment deux trones principaux, l'un antérieur, l'autre postérieur, et se confondent en un seul, qui vient s'ouvrir dans le fond de l'oronane de Bojanus (5), vers son extrémité

(1) Pl. 8, fig. 4 .

(2) Observations sur la struclure et les fonclions de quelques Zoophyles, Mollusgues et Crustacés des cótes de la France (Ann. des sc. nat., $3^{\mathrm{e}}$ série, t. XVIII, p. 321 ).

(3) Pl. 8, fig. 3.

(4) Pl. 8 , fig. i $(a)$.

(5) Pl. 8 , fig. 4 (b). 
antérieure. Il y a répétition de la même disposition pour chacun des còtés du corps, en sorte qu'iei il y a fuatre glaudes, deux ovaires, et deux testicules, mais il n'y a que deux orifices génitaux, répondant ì deux systèmes de canaux, dont les ramifications se répandent à la fois dans la glande mâle et dans la glande fenelle. Comme la marche des canaux est oblique, comme aussi la séparation des glandes est brusque et nette, il arrive que vers la ligne de démarcation, ì côté des acini fenelles, viennent s'insérer sur le même ramuscule excréteur des acini mâles; el la conséquence forcée de cette disposition est le mélange des deux produits de la sécrétion avant leur sortic par le même orifice.

De Blainville avait entrevu cette position, et cela parce qu’il se faisait une idée fausse du sexe en général; il regardait, en effet, ces animaux comme étant hermaphrodites, et, pour celte raison, il pensait que l'ovaire devait être plus profondément situé que le testicule, afin que les germes fussent arrosés par la liqueur fécondante à leur sortic. C'est une chose singulière que de voir des erreurs condure quelquefois à la vérité; nous allons encore en trouver un exemple pour les Pandores.

J'ai rencontré sur des Peignes glabres de Cette de petits îlots de glandes femelles isolés (1) au milien de la glande mâle. Leur couleur les faisait reconnaitre bien vite. Ceci a déja été inchiqué par les anteurs, par M. Humbert en particulier, qui a observé des faits analogues dans la même loealité; je n’ai jaunais rencontré cette particularité sur la même espèce que je pêchais si abondamment à Mahon et que j'y ai examinée si souvent. Dans ce cas, le mélange des oufs avec le sperme se fait presque à la sortie des acini. J'insiste sur eetle disposition des canaux exeréteurs dans les Peignes, parce qu'elle a été complétement méconnue. Siebold dit : "Les conduits excréteurs n’ont pas encore été représentés d'une manière satisfaisante (2), » et M. Humbert (3), dans sa note, déclare n'avoir pu en reconnaître la marche. Je dois dire eependant que

(1) Pl. 8, fig. $4(d)$.

(2) Analomie comparée, p. 286, t. I, trad. franç.

(3) Humbert, Note sur la structure des organes générateurs chez quelques eqpèces du genre Pecton (1nn des sc nat., $3^{\text {e }}$ série, I XX, p. 333). 
Garner 1), dans un travail que j'ai déjà eu l'occasion de eiter souvent, a reeonnu dans quelques espèces la place de l'orifice génital. La difficulté que l'on éprouve à pouvoir suivre les conduits est des plus grandes, et les vaisscaux sanguins, qui sont très apparents et marchent parallèlement à eux, rendent les erreurs très faciles à faire. Ceux-ei sont plus superfieiels, tandis que les autres ne se rencontrent que dans une eouche profonde. Dans tous les cas, les injeetions servent, quand elles sont poussées assez loin, à bien établir la différence.

Dans ce second exemple, la disposition, tout en ayant beaucoup de rapport avee la précédente, offre eependant quelques particularités qui méritent une mention toute spéciale.

La couleur vive de l'ovaire manque dans les Pandores, et la position du testicule n'est plus la mème; enfin les orifices des deux glandes sont distinets et séparés. Cette dernière différenee aurait dù nous faire étudier les Pandores avant les Peignes, comme faisant plus directement le passage entre les diöques et les monöques. Si j'ai suivi un ordre inverse, e'est que la coloration, très marquée dans les Peignes, facilite beancoup la distinction des glandes.

Le testicule est placé sur les côtés du foie, l'ovaire est en arrière de lui, et un peu dans la base du pied. Ce changement de position est, on le comprend, une conséquence d'une organisation générale tout autre dans les Pandores que dans les Peignes. Tandis que le pied est, dans un cas, un appendice très grêle placé en avant d'une masse viscérale considérable, dans l'autre il est en forme de lame et se continue avec cette masse peu développée jusqu'au muscle postérieur des valves. Il faut eependant remarquer qu’ici comme dans les Peignes, la glande mâle est antérieure à la glande femelle, mais elle n'est pas plus extérieure que cette dernière. En sorte que l'opinion de de Blainville, basće sur des idées générales préconçues, et qui se trouvait justifiée par les premières espèces monoïques, est contredite par les secondes.

Je n'aurai rien à ajouter pour la texture; les spermatozoïdes pt

(1) The Magazine of natural history de Charlesworth, 1839, p. 394 et suiv. Il y a des erreurs toutefois que je ferai remarquer en parlant des orifices. 
les auls se développent dans des eceuns qui terminent les ramifications des eanaux de la glande. Le parenchyme cellulaire des cocums ressemble en tout point à celui des autres Acéphales.

La disposition offre quelques particularilés : le testicule se remarque avec beaucoup de netteté sur le foic; il y forme des arborisations d'une élégance extrême, dont la blancheur ressort sur le fond brunâtre du foie. Ces deux conditions, s’ajoutant à un écartement assez grand des rameaux, permettent d'étudier le testieule avee beaucoup de facilité.

En traitant des diö̈ques, j’ai montré que les formes des éléments de l'organe mâle pouvaient se rapporter à deux types principaux; que les cœeums sécréteurs formaient, tantôt de longs tubes plus ou moins ramifiés sur les côtes, tantôt des amas de granulations donnant l'apparence parfaite d'une grappe. Le testieule de la Pandore se rapporte au premier type, et se rapproche, par conséquent, de celui des Vénus; mais il en diffère en ce que l'extrémité des culs-de-sac séeréteurs est un peu plus renflée.

Les conduits de l'ovaire ne présentent rien de remarquable dans leur profondeur; mais dans le voisinage de leurs orifices ils sont très dilatés, et forment de véritables ampoules.

Quant aux orifices de la génération, ils sont distincts et séparés, placés l'un à côté de l'autre à la racine de la masse abdominale, vers l'extrémité antérieure du sac rénal et annexe; ils ont la forme de deux boutonnières placées parallèlement l'une à côté de l'autre. Ce fait mérite toute notre attention, ear voilà sur un même individı hermaphrodite une disposition anatomique qui entrâne le mélange des produits de la génération hors des glandes; et à l'égard de celte séparation il n'est pas possible qu'il y ait eu erreur de ma part, ear les dernières portions de l'oviducte étant dilatées et remplies d'ceufs, je pouvais par de légères pressious causer une ponte forcée. De même pour la liqueur séminale, elle s'écoulait toujours avec facilité par l'orifice le plus antéricur.

Cette séparation des deux orifices est un lait que je n’ai vu signalé par aucun auteur. Il prouse cucore mieux que ne l'avaient fait les Peignes rue rlacune des parties sexuelles de l'animal représente eomplélement un individu de l'un des sexes des Icéphales diöques. 
Je ne puis n'empêteher, à l'oecasion des Pandores, de revenir sur le travail de II. Deshayes.

L'auteur est dans la rérité en disant que ces Mollusques sont hermaphrodites; on va voir cependant que e'est le hasard qui le ronduit à cette conclusion. J'ai déji cité le passage (1) auquel je fais allusion; maintenant on pourra mieux eomprendre que «l'organe »des crochets, qui fait une sorte de bordure aux organes le long » du bord dorsal, »ne peut être considéré comme un testicule $(2)$. "Les petits amas blane jaunâtre formant la glande sont rappro" chés, mais isolés les uns des autres, et ne semblant avoir entre eux » de communication que par le tissu parenchymateux dans lequel ils » sont englobés. „Ce n'est pas là le cas de la glande mâle dans laquelle on voit si nettement les conduits excréteurs, portant ì l'extrémité de leurs ramifications les petites ampoules ou coccums sécréteurs. La deseription et les figures que l'auteur donne des spermatozö̈des montrent que bien certainement ce ne sont pas les filaments spermatiques qu'il a vus; il ne les a observés qu'à un grossissement de 250 diamètres, et il a reconnu qu'ils sont formés par des granules se prolongeant en une queue courte et très pointue. Les figures indiquent ì un aussi faible grossissement une taille beaucoup trop grande pour des spermatozoïdes. La position de la glande mâle, sa disposition et sa texture, ainsi que la deseription des zoospermes, tout montre que le testicule n'a pas été obscrvé. Vais comme l'auteur eroit, en général, à l’hermaphrodisme, il arrive, par une heureuse coüncidence due au hasard et ì l'erreur, à cette conclusion vraie : « Dans les Pandores, les deux organes de la génération sont »nettement séparés. ”

En résumé, glandes en tout semblables à celles des Acéphales diö̈ques, mais réunion des deux sexes dans le corpss d'un même. animal; distinetion nette et parfaite des éléments testiculaires ef ovariques : tels sont les caractères de ce groupe, qui s'est présenté peu nombreux en espèces dans nos observations.

(1) Voyez la partic historique au commencement du travai! sur les organce. génitaux des Icéphales (Annal., P. 163, t. II, ann. 1854).

(2) Deshayes, Exped de lidgerie, Mollusques, p. 2:20, pł. XXIT. 
Ainsi se concilient les opinions de MIM. Edwards et de Siebold. Ces deux savants étaient l'un et l'autre dans la vérité, ils avaient seulement observé des espèces différentes: les unes hermaphrodites, les autres uniscxuées.

SECONDE DIVISION.

Acéphales monö̈ques à glandes confondues.

Dans celte division des monö̈ques, on trouve une espèce de Bucarde et les Huitres.

Le Cardium hermaphrodite qui s'est offert à l'observation est assez commun sur les côtes de la Nanche et de la Bretagne. II se trouve au niveau des plus basses marées, au milieu des herbages ou prairies marines formées par les Zostères. J'ai trouvé nıoi-même ceux que j'ai étudiés au milieu du groupe des Hébiens, près d'une île que les habitants de la côte appellent la Colombière; les pêcheurs de Soles et de Turbots en rapportent aussi du fond après avoir trainé leur chalut sur les herbages. Il y a eu quelques confusions sur l'espèce; je les signalerai afin que l'on se trouve averti. J'espère qu'il n'y aura pas de doutes avec les indications de la localité où je l'ai observée.

Deux espèces ressemblent beaucoup, d'après les planches et les descriptions, à la coquille dont il s'agit : ce sont le Cardium serratum (1) et le Cardium lavigatum (2). D'après Bruguière (Encyclopédie méthodique), la première espèce se trouve sur les còtes de l'océan d'Angleterre; la seconde appartient aux mers des Antilles. Ce serait à tort, dit cet auteur, que l'on penserait avec le baron de Born que la dernière vient aussi sur les côtes d'Angleterre. Ce qui aurait eausé l'erreur de Born, e'est que Pennant a appelé C. lavigatum le $C$. serratum. D'après Forbes et Hanley (3), ce serait le $C$. norvegicum qui vivrait sur les côtes d'Angleterre, el

(1) Brug., Encycl. méth., t. I, p. 229, n 27, fig. 2, pl. 299. - Lamk, l. VI, p. $401, \mathrm{n}^{\circ} 25$.

(2) Id., p. 231, n 30, fig. 2, pl. 300. - Lamk, t. VI, p. 403, $\mathrm{n}^{\circ} 25$.

(3) Histoire des Mollusques d'Angleterre, t. II, p. 33 et 3s. - Leur C. norvegicum (Sprengles) est celui que Pennant a nommé $C$. lavigatum, que Lamk désigne sous le nom de $C$. serratum. Il est commun. Leur $C$. serratum, placé à la fin du genre comme spurious, est une West-Indian shell. 
cela doit faire supposer que e'est le même qui vit sur les plages de Bretagne.

Quoi qu’il en soit, ces deux espèces sont très voisines, et j'ai trouvé dans la collection de la Sorbonne plusieurs échantillons des corpuilles dont il s'agit, ayant servi ì M. de Blainville pour faire son Manuel de malacologie, et annotées de sa main, lantôt sous le nom de lavigatum, tantôt sous cclui de serratum, toujours comme étant fort communs sur les côtes de la Nanche.

J'ai insisté sur cette distinction spécifique, parce qu’il me parait important et nécessaire, après les doutes nombreux, les discussions qui se sont élevées sur le sexe des Acéphales, de bien déterminer de quel animal il était question. Oı a vu que pour les Peignes, les auteurs, quoique dans le vrai, semblaient cependant en désaccord, justement parce qu'une espèce sortait de la règle générale à laquelle était soumis le genre.

La glande génitale des Bucardes dont il est iei question est disposée comme dans les autres espèces du même genre. Elle remonte sur les côtés du foie, et s'étend dans la cavité viscérale qui оссupc la base du pied. Ses ramifications s'engagent au milieu des brides fibreuses et musculaires, où on les reconnaît facilement à une teinte un peu grisâtre, et aux arborisations qui rappellent un peu, soit le testicule des Pandores, soit la glande màle des Vénus.

Toutes ces dispositions générales sont semblables à celles qui ont été déjà indiquées, et il est inutile d'y revenir.

La texture de cette glande génitale est bien importante, car elle aide à arriver à une conclusion sur la sexualité des Huîtres, si difficile à bien établir.

Quand on enlève un lambeau de la glande en tirant sur un canal cxcréteur, et qu'on le soumet au microseope, on voit avec la dernière évidence des culs-de-sac des cœcums latéraux plus ou moins ramifiés, plus ou moins courts, plus ou moins éloignés, insérés tout le long d'un tube sécrétcur. Ainsi pas de différence pour la disposition des acini; mais en portant son attention sur le contenu des coecums, on ne tarde pas à remarquer que les uns sont remplis d'oufs, les autres de sperme, et que quelques-uns renferment ì la fois les deux. 
En poussant les investigations plus loin, on ne tarle pas à recommaitre que le parenchyme de ces culs-de-sae est cellulaire, et que les aufs et les spermatozoïdes se développent dans le Cardium serratum exactement comme dans les autres $\Lambda$ céphales ; mais senlement nous trouvons cette différence capitale et du plus haut intérêt pour la philosophic anatomique, que le même cul-de-sac peut être en partic mâle, en partic femelle, ou bien que deux cœcums de sexe différent peuvent être insérés à côté l'un de l'autre sur un même conduit excréteur.

On retrouve done ici dans un Mollusique acéphale quelque chose de complétement analogue à ce qui s'observe dans les Gastéropodes, Aplisie, Limace, Itélix, ete.

Je reviendrai plus loin sur celte disposition curieuse, qui nous montre, au milieu d'un groupe bien earactérisé par la distinction des sexes, une espèce faisant exception.

Les observations ont porté sur cinq individus pris dans les conditions les plus naturelles; que s'il arrivail, ce qui me paraît peu probable, que par la suite un examen plus étendu fit reconnaître l'unisexualité, il resterait au moins aequis ce fait: que les Acéphales dioïques peuvent accidentellement se présenter à l'élat hermaphrodite. Je erois que eet état est vraiment l'état habituel de l'espèce qui nous occupe.

On sait à quelles nombreuses discussions le sexe des Huîtres a donné lieu; les opinions les plus opposées se sont tour à tour trouvées en face, et c'était pour avoir une idée plus nette de la question que j'avais entrepris des recherches. Mais pendant mon voyage aux îles Baléares et en Espagne, M. Davaine (1) me devancait par une publication étendue sur le même sujet. J'ignorais complétement les résultats obtenus par lui, quand j'arrivais exactement aux mêmes conclusions seulement en ce qui touche l'état de la glande génitale de l'Huìtre, qui paraît tantôt mâle, tantôt femelle, lantôt hermaphrodite; quant à l'interprétation de ces états, elle est différente, à mes yeux, de celle qui a été donnée dans le travail que je rappelle. Toujours est-il que cette concordance entre les résultats obtenus par deux observateurs employant un long temps it

(1) Mémoires de la Sociélé de liologie, $18: 3$. 
l'élude du mème fait, sans avoir connaissance de leurs travaux, permettra aux naturalistes de considérer désormais la question du sexe de l'Huitre comme résolue; la priorité, si priorité il y a, à déclarer les Huîtres hermaphrodites après que tant d'auteurs ont soutenu cette opinion, en revient ì M. Davaine.

Je bornerai là les apprécialions historiques; l'analỵse des travaux de Martin Lister (1), de sir Everard Home (2), de Quatrefages (3), le Forbes et Hanley (4), ete., ne présenteraient que des opinions opposées tantòt pour la réunion des sexes, tantôt pour leur séparation.

Mes observations ont porté sur les Huitres de la Méditerranée et de l'Océan. J'ai cxaminé, en passant à la Rochelle, quelques Huîtres de Marennes; à Paris, celles qui sont apportées sur les marchés des côtes de la Mlanche; mais les études suivies ont principadement été faites sur la grosse Huître pied-de-cheval, sur l'Huître comestible ordinaire de Mahon et de Cette, sur celle de Saint-Jacut-la-Mer yui se développe autour des Hébiens, et surtout sur la petite IIuitre dentelée ou stentine (5) qui abonde dans le port de Mahon.

La glande génitale de l'Huitre est placée dans les parties latérales et dorsales du corps, tout le tour du foie. Si elle est plus développée dans la partie antérieure que dans les autres Acéphales, e’est que l'abdomen est rudimentaire en avant et en dessous du musele des valves Les rapports, du reste, sont les mêmes que dans les exemples précédents, et je n'ai rien à ajouter à cet égard.

La glande est sans couleur; cependant il arrive assez somvent que, lorsqu'elle est gonflée par les produits de la sécrétion, elle est un peu jaunâtre.

La structure est très difficile à observer, comme, du reste, tous les autres points d'anatomie de l'Huitre, et l'on n'arrive à s'en faire une idée exacte que par des recherches comparatives. Geci

(1) M. Lister, 1678, Londres, Historice animalium Anglice tres tractus.

(2) Sir E. Home, Recherches sur la manière dont se fail la propagation dans: l'Ifuitre commune (Transact. phil. de Londres, 1827).

(3) De Quatrefages, Comples rendus, t. XXVIII.

(i) Forbes and Hanley, Histoire des Mollusques des cótes de l'Angleterre.

(5) Ostrea stentina, Lamk, 2" edit., t. VII, p. 336, n० 30. 
nous explique comment les auteur's qui n'ont pas cherché de point de départ comparatif ont fait des erreurs grossières, même d'anatomie descriptive. Quand on coupe le eorps de l'animal, on est frappé de la densité de la glande génitale, dont la couleur pâle blane jaunâtre se distingue nettement des lobules du foie qu'elle entoure; on y voit, à l'aide de la loupe, de petits amas qui rappellent les lobules primitifs et les acini des Peignes variés, des Huitres vermeilles, ete. Et il n'y a pas le moindre doute à avoir ; les acini, malgré la confusion des éléments, sont bien la terminaison en eulde-sac des canaux excréteurs. J'ai trouvé un exemple dans un état analogue à celui que j’ai indiqué pour le Pecten varius; le parenehyme de la glande était réduit à de petites masses placées aux extrémités des dernières ramifications des canaux, dont la disposition rappelait beancoup celle des espèces précédentes, avec cette différence toutefois, en rapport avec l'avortement de l'abdomen, qu'il n'y avait qu'un seul canal excréteur principal et antérieur.

Mis sur la voie par cet heureux exemple, j’ai pu reconnaitre l'orifice de la génération (1) et la marche des vaisseaux qui y aboutissent. On ne trouve qu'un orifice de chaque côté du corps ; il est placé en bas et un peu en avant du muscle des valves; on ne le découvre qu'avec beaucoup de difficulté, parce qu'il est entre les deux cordons nerveux, qui vont : l'un, interne (2), du ganglion branchial au ganglion buccal; l'autre, externe (3), du ganglion branchial à la branchie. Ce rapport de l'orifice génital avec le nerf connectif fournit un point de repaire précieux ; ear, en suivant le cordon nerveux, un arrive, comme pour les autres Acéphales, à l'orifice de la génération. Cette analogie montre la réalité de la position. On pourrait peut-être éprouver quelques difficultés, si, après avoir coupé les branchies, on ne faisait attention que le nerf branchial, plus apparent, très voisin du connectif, doit être en dehors. Mais jamais on ne manquera d'arriver dans le canal, si l'on fait glisser, d'arrière en avant, entre les deux nerfs, une epingle fine en présentant la tête la première. Le trone principal du canal se dirige

(1) Pl. 8, fig. $5(b)$.

(2) Pl. 8 , fig. $5(x)$.

(3) Pl. 8 , fig. $5(n)$. 
vers le dos en décrivant une courbe en avant et en bas de l'excilvation du péricarde. Vers son bord inférieur, et puis sur les deux côtés, se rendent les canaux secondaires venant de toutes les parties de la glande. Ce canal devient évident quand on y pousse une injection; mais le point difficile est de trouver l'orifice, et e'est à cette difficulté qu'il faut rapporter les erreurs commises par les auteurs.

Ainsi, sir Everard Home (1) décrit un oviducte, unique pour les eleux glandes, placé sur la ligne médiane, remontant vers la bouche, non loin de laquelle il s'ouvre. Une telle erreur est difficile. à comprendre; car le dessin qui accompagne le texte montre un orifice et un oviducte, pour le moins anssi grand que l'intestin.

Quant à II. Davaine, il est plus généreux que E. Home; il indique pour lecôté droit, par des lignes ponctuées, trois pertuis (2); comme l'on doit penser qu'il admet le même nombre de chaque côté du corps, cela fait six orifices génitaux, qui ne sont visilbles, dit-il (3), qu'au moment de la ponte. Ceci prouve, sans aucun doute, que les deux seuls et véritables orifices ont été méconnus; car, dans l'hiver, sur les Huîtres à peu près infécondes que l'on apporte à Paris, on peut reconnaitre, sans qu'il puisse rester de doute, la fente génitale en forme de boutonnière. M. Davaine ne dit pas, il est vrai, qu'il existe six orifices; mais ce nombre est une conséquence forcée de son travail : car ou bien il rejette la symétrie (il faudrait en donner les raisons), et l'on n'a que trois orifices à droite, ou bien il l'admet, et l'on est conduit à considérer le côté gauche comme semblable au côté droit, qu'il a dessiné planche 1, figure 1. Ce dilemme conduit des deux côtés à des conclusions fausses.

Il est fìcheux de rencontrer une telle erreur d'anatomie descriptive sur un fait relativement facile au début d'un travail où les questions les plus ardues d'anatomie, de structure et d'embryogénie, doivent être abordées.

En résumé, les orifices indiqués par sir E. Home et parr M. Da-

(1) Cronian lectures for 1826, dans Philosophical Transactions of the royal Sociely of London, 1827, p. 42.

(2) Mémoires de la Société de biologie, t. IV, 1853, pl. I, fig. $2(k)$.

(3) Id., f. 30 \&. 
vaine doivent ètre considérés comme des déchirures, et la position exacte que nous indiquons est un fait nouveau resté inaperçu jusqu'ici.

Quant à la texture intime de la glande génitale, on l'observe avec d'autant moins de difficulté que l'un des deux sexes prédomine davantage. Le caractère desHuitres, et de la division des Acéphales où nous les plaçons, est précisément le mélange, la confusion la plus complète des deux glandes sexuelles; aussi, quand on prend une portion du parenchỵme abdominal, trouve-t-on presque toujours dans le champ du mieroscope des spermatozoïdes et rles oufs.

Ceux-ci se distinguent facilement par leurs contours nets, l'opacité du vitellus et l'éclaircie qui, vers le milieu, correspond ì la vésicule transparente. On rencontre autour de l'ceuf une enveloppe mince, largement ouverte, qui est évidemment la capsule (1); ‘uand il n'est pas bien développé, on trouve des vésicules transparentes en assez griand nombre pour ne point faire de doute sur l'élat femelle.

Less spermatozoüdes (2) sont de ceux dont la tête est globuleuse, légèrement conique en avant. Leur taille n'est pas considérable; ils sont un peu aplatis et convexes sur leurs faces. Quand ils sont complétement libres, ils ont une vivacité qui les rend difficiles ì suive; mais, comme on les trouve le plus souvent réunis en paquets (3), on peut les étudier, surtout quand ils cherchent à s'isoler. Les têtes forment des masses assez régulièrement ovoüdes entourées d'un espace clair; celui-ci est dù à l'éloignement des granulations par les mouvements ondulatoires des yueues qui hérissent de toutes parts cette agglomération. La disposition que nous indiquons iei est très caractéristique et loute particulière àl'Huître.

Voilà les produits de la sécrétion; où et comment sont-ils formés? Je disais que plus un sexe l'emporte sur l'autre, plus la distinction des éléments est facile à faire : dans un cas où l'Huìtre était presque complétement mille, j’ai trouvé des culs-le-sae, de véri-

(1) Pl. 8, fig. 6 .

(2) Pl. 8, fig. 9

(3) Pl. 8, fig. 8 . 
tables acini (1, yui ressemblaient tout à fait à eeux des Lantellibranches diö̈ques. Dans leur intérieur, on remarquait des lignes indiquent vaguement les paquets de spermatozoïdes. Les cellules, analogues à celles où l'on a vu se développer les filaments, se retrouvent ici avec une identité absolue de caractères; mais elles sont réunies en masses (2), tout à fait semblables à celles des filaments, même avant que ceux-ci soient développés. Jamais je n'ai vu ces amas entourés d'une membrane pouvant indiquer une cellule mère, bien que la disposition donne à supposer son existence. Toujours est-il qu’icion voit, avec la dernière évidence, la relation qui existe entre la partie productrice et la partic produite. Il est done évident que, dans les acini mâles, la production des spermatozoïdes a lieu comme dans les autres Acéphales; mais seulement que le parenchyme se trouve divisé en pelites masses, correspondant par arance aux paquets des filaments.

Pour l'œuf, il doit en être aussi de même. La capsule enveloppante nous conduit à admettre que e'est dans la paroi du tube sécréteur qu'il se développe, pour faire ensuite saillie dans l'intérieur, et pour y tomber quand, peu à peu, celle-ci s'est détruite. L'œuf, devenu libre lorsqu'il est pondu, n'est jamais entoure d'une zone transparente.

Voilà pour la glande où l'on trouve des acini mâles et des acini femelles assez distincts ; mais quand il y a une fusion telle, que l'on a toujours, quelques précautions que l’on prenne, mélangé ensemble des neufs et des spermatozoüdes, quels sont les rapports de r'es éléments? Je dois avouer rue, malgré des recherches les julus atlentives, je n'ai pu voir si le même cul-de-sac, les mêmes acini, produisaient à la fois des spermatozoüdes et des culs; ou si toujours les acini mâles étaient séparés des acini femelles. Rien ne s’opposerait à la première opinion, ear dans les Limaces, jar exemple, il en est ainsi. On verra, dans la comparaison générale de l'ovaire et du testicule, que celte manière de voir peut être sontenue aree quelque raison.

(1) Pl. 8, fig. 7. L'indication des masses est trop maigre, les lignes sont Irop fortes.

(2) Pl. 8, fig. $8(a)$. 
Mais la meilleure preuve est ce qui s'observe dans le Cardium hermaphrodite dont il vient d'être question. Là, en effet, il n'y a et ne peut y avoir de doute; un même cul-de-sac sécrète à la fois les deux éléments sexuels, et tout porte à croire que si la démonstration n'est pas absolue pour l'Huître, elle doit cependant lui être applicable. Comme tous les points d'organisation présentent une certaine difficulté à être bien constatés dans cette dernière espèce, on comprend que pour un fait d'une telle délicatesse on soit un peu embarrassé à arriver ì une détermination exacte sans le secours des faits comparatifs.

Je ne sais si le moyen employé par M. Davaine pour éludier la texture intime peut l'avoir bien servi ; les dessiccations auxqquelles il a eu recours ne me semblent pas faites pour lever les diffieultés. Comment, dans des lissus aussi mous et aussi abreuvés de liquide, peut-on arriver à obtenir un corps solide conservant assez sa forme, pour qu'en observant ensuite des tranches minees, on ait une idcée exacte des parties? Les aréoles, les loges tantôt vides, tantòt l'emplies de spermatozoïdes et d'œufs, qu'il déerit, paraissent ètre les lacunes de l'abdomen formées par l'entrecroisement des faisceaux fibreux et musculaires, dans lesquelles viennent se loger les acini, ou extrémités des tubes sécréteurs, qui ont ćchappé à son observation.

D'apres les recherches de cet auteur, « les zoospermes forment "la couche interne, et les ovules la couche externe ou envelop"pante (1). "On aurail done deux parties emboîlées l'une daus l'autre; la partie femelle entourerait la partie mâle. Je erois qu'il y a là une erreur due au morle d'observation. Les spermatozoüdes tombent après lenr sécrétion dans l'intérieur du canal, mais à ce moment ils ne font déjà plus partie du testicule; aussi, dans cette deseription de la texture de la glande, l'auteur paraît avoir pris le produit de la sécrétion pour le parenchyme sécréteur. Du reste, la dessiccation ayant fait disparaitre les parois du crenm sécréteur, on comprend qu’il était difficile d'arriver à un résultat conforme aux fails que l'étude comparative d'animaux non desscechés a pu nous fournir. On comprend aussi comment les oufs, que l'on a vus, dans

(1) Loc. cit, p. 315. 
d'autres exemples, lixís aux parois dı tube séeréteur, sont plus externes que les spermatozoïdes; mais cela n'indirue pas le moins du monde les rapports des éléments séeréteurs de la glande.

Comment expliquer les différentes opinions des auteurs au sujel des sexes de l'Huître? Je crois inutile de les rapporter toutes, ear elles se résument dans une affirmative pour ou contre l'hermaphrodisme; affirmative qui a eu le plus souvent pour cause lit manière dont l'auteur envisageait le sexe des Acéphales en général ; et je crois avoir donné assez d'étendue à cette partic historique pour n'avoir point à y revenir ici.

La cause de ces divergences tient à ce que tantôt l'Huître a l'apjparence hermaphrodite, tantôt l'apparence unisexuée. Mais faut-il croire avec M. Davaine (1) que ces élats sont la conséquence des changements successifs qui se passent dans la glande ? Suivant lui, le sperme se développe le premier (apparence mâle); viennent ensuite les oufs (apparence hermaphrodite); enfin la fécondation s'opère, les spermatozoïdes disparaissent, les nul's restent seuls (apparence femelle) (2). Les preuves à l'appui de cette opinion sont tirées de ce fait plyssiologique que, lorsque la fécondation est accomplie, les spermatozö̈les devenus inutiles disparaisserit, et de ce que aussi la glande, en se développant de nouveau, commence son évolution par la glande mâle, qui marche parallèlement au développement des embryons.

D'après lui, lorsque la ponte a eu lieu, a ou lorsque les aufs "pondus n'ont encore subi que les premières phases du fraction»nement, on ne reneontre dans la glande ovospermagène ni » zoospermes ni ovules en voie de formation. En général, des cel"lules spermatogènes se montrent dans eette glande, lorsque les " ovules contenus dans le manteau commeneent à ètre pourvus de » cils vibratiles.... « (Lorsque les embryons contenus dans la eavité s incubatrice ont un appareil de matation distinct, la glande ovo"spermogène contient toujours des cellules spermatogènes et des » masses de zoospermes bien earactérisées. Enfin à l'éporque où les »embryons sont rejetés du manteau de l'Huitre mère, les masses

(1) Loc. cit., p. 309 .

(2) Loc. cil., p. 316 
"de zoospermes sont nombreuses, bien développées, et ont acquis » des mouvements très vifs. „ Ceci semble indiquer que lorsure lit ponte a eu lieu, l'activité des glandes recommenee.

Ces raisons sont loin d'être convaincantes; quand ou trouve des Huîtres presque complétement mâles, dont tout le corps est gonflé de sperme, on se demande où prendront place les oufs qui se développeront eux aussi en quantité énorme. Certains exemples m'ont paru être rendus aussi turgides par les éléments femelles que les autres l'étaient par le sperme; rien ne prouve que la fécondation entraine la cessation de la séerétion spermatique, et la disparition de tout le fluide mâle est pour le moins hypothélique. Quand elle serait démontrée, elle n’aurail lieu qu'au moment où les reufs sont mûrs, et aptes à être fécondés; avant cetle éporjue, cenx-ci ont déjì une taille si grande, et sont en nombre si considérable, que je ne comprends pas la pơssibilité de les voir se placer dans une glande déjì remplie, dans quelques cas, jusqu'aux dernic̀res limites, par le produit de sa séerétion.

Quaut ì démontrer la sucession du développement des glandes par l'élat plus ou moins avancé du développement des jeunes restant dans le manteau de leur mère, cela me parait absolument impossible. Cart, d'une part, il y a des variations dans le développement des glandes, tenant ì la température, à la localité et à l'individu: cela est si vrai, que l'on trouve des Huitres en gestation pendant prìs de deux mois. De l'antre, les plases d'évolution des embryons sont trop peu lixes pour servir à résoudre la question; il est des moments où elles marchent arec une rapidité extrême, tandis que dans d'autres elles deviennent presufue stationnaires. J'avais eu, en 1851 (aoùt), l'oceasion de chereher ì Cette quelques faits sur le léveloppement de l'Aplysie; l'une des périodes passait avee une telle rapidité, que les ceufs recucillis à la sortie de l'oviduete, dans le port, avaient déjà franchi cette période quand j'étais rentré pour les examiner. En 1853 (fin de septembre), au contraire, mais dans un autre moment, je ne reneontrais que cet état devenu presque stationnaire. Il me paraît done difficile de prendre pour mesure du développement de la glande l'échelle d'évolution de l'œuf.

Je suis loin de vouloir dire rue la sécrétion du sperme el de l'œuf 
marche absolument en même temps, je erois mène que le sperme doit arriver plutôt que les aufs au terme de son développement; mais entre cette opinion et celle qui consiste à montrer la glande produisant d'abord du sperme, cusuite des oufs, il y a une immense différence. D'une part, tandisque les exemples du Pecten, du Cardium hermaphrodite sont lì pour appuyer ma manière de voir, de l'autre les faits ne sont nullement en rapport avee l'explication des états successifs de la glande hermaphrodite. Celte explication, si l'on en tirait les conséquenees naturelles, ne tendrait ì rien moins qu'à montrer la même glande jouant suceessivement le rôle de mâle et de femelle. Pour admettre une telle chose, il faut des preuves eontre lesquelles nulle objection ne puisse s'élever.

Mais il est un fait qui frappe dans celte étude, e'est le nombrr relativement très considérable de mìles. Les femelles, an contraire, semblent très rares, de l'areu même de .1!. Davaine qui II'en a rencontré que deux sur trois cents lluitres dans une première série de recherches, et dans une seconde la proportion n’a pas cété plus forte (page 310 , alinéa $f$, loc. cit.). Si les différents aspects de la glande étaient dus aux changements suceessifs indiqués, on ne voit pas pourquoi l'état complétement lemelle ne serait pas plus fréquent. Comment expliquer eette multiplicité des mâles? Dans presque tous les cas les animaux pondant, ou ayant des larves dans leur manteau, sont remarquablement caractérisés comme mâles. Il faut attribuer cela à la sortie des œufs, qui a laissé le testicule plus apparent; car, dans la plupart des exemples, la glande est flétrie après la ponte, et eependant clle renferme des spermatozoïdes très bien développés et très vifs. J'ai fait encore la même observation à Saint-Jaeul-la-Mer en Bretagne, les spermatozoïdes que je reneontrais dans tous les points de la glande éticicut trop développés pour être dus à une nouvelle sécrélion, ayant eu lieu pendant l'ineubation des œufs. L'aspect particulier de la glande ne pouvait laisser de doute; il n’indiquait pas du tout une nouvelle activité, une nouvelle érolution.

Dans l'opinion de M. Daraine, il fiul admettre, pour explirfuer ce lait, que, dès que la ponte a eu lieu, la glande mâle reprend ses fonctions avec une promptitude extrêne, et sécrète de nouvean le. 
sperme; en preuve le développement simultané de l'cubryon et du testicule. Le plus souvent, je ne crois pas qu'il en soit ainsi, et que la reproduction ou l'évolution des glandes se fasse d'avance; en général, elle ne commence qu'au printemps.

Si le testicule recommence son évolution, peudant que l'embryon se développe, on est conduit à se demander ce que devient le produit de la séerétion dans l'intervalle de deux pontes. La sortie des neufs n'a lieu, comme on le sait, que pendant l'été, et il faut admettre dans l'opinion que je eritique, ou bien que le sperme sécrété ì la fin d'un été est conservé dans la glande pour servir à la fécondation des oufs dans la saison prochaine, ou bien qu’il est rejeté. Cette dernière supposition n'est guère admissible; on ne comprend pas, en effet, qu'une glande sécrète inutilement un produit sans usage. Reste done la première, dont la conséfuence forcée est la présence du sperme bien caractérisé dans l’intervalle de deux périodes de reproduction. II. Davaine est muet à cet égard; il n’indique pas du tout dans quel état il a trouvé les Huitres pendant l'hiver, pendant l'époque où la plupart des Acéphales ont leurs glandes génitales à l'état de repos ; et où les tissus des glandes, bien que renfermant quelquefois des éléments déjà caractéristiques, ne présentent presque jamais des produits nettement caractérisés, comme la deseription des spermatozoüdes, an moment où l'embryon de l'Huitre va quitter le manteau de sa mère, l'indiquerait.

Après bien des recherches, j’ai fini par retrouver, dans les animaux qui semblaient seulement femelles, toujours des zoospermes 'ui étaient dans la partie rulimenlaire, représentant la masse abelominale au-dessous du musele des valves. Ce point, très restreint, oì semble être relégué le peu de testicule qui existe dans ces cas, n'aurait-il pas échappé à M. Davaine ; et quand il aurait cru avoir un exemple où le sperme avait disparu en fécondant les oeufs, n’y avait-il pas encore des zoospermes qu’il n'avait pas trouvés? A-l-il recherché le sperme dans le point que j’indique sur le millier d'Huîtres qu'il a observées?

Pour nous, il parait démontré que les glandes se développent dans des proportions variables; que lel individu est plus femelle que mâle, que tel autre est plus mâle que femelle; enfin que, dans d'autres 
cas, il y a égalité. Les faits comparatifs viennent à l'appui de cetle nuanière de voir : ainsi, dans le Pecten glaber de l'étang de Thau, près de Celte, j'ai rencontré, ainsi que l'a vu M. Humbert (1), des individus ayant des îlots de glande femelle placés au milieu de la glande màle, ou inversement. N'est-il pas évident que, dans ces cas, la tendance de la nature était le mélange des éléments, et que la proportion d'une glande relativement à l'autre devenait plus grande?

Ce qui cause l'opinion de M. Davaine, e'est qu'il ne peut eroire ¡que, dans quelques cas, l’Huître soit sinon complélement unisexuée, du moins presque entièrement unisexuée; et cela parce qu'il serait conduit à admeltre « une condition encore inconnue. » Or il est une observation de M. Humbert qui peut lever ce doute; elle est, par elle-même, très curieuse et très intéressante (2). Le Pecten glaber, dont l'hermaphrodisme est incontestable et incontesté, le même que je citais il n'y a qu'un instant, s'est présenté deux fois complétement unisexué. Un individu était entièrement mâle, et l'autre était tout ì fait femelle, sauf un lobule blanc lesticulaire. N'y a-t-il pas dans ce fait très remarquable un enseignement qui nous démontre, dans une espèce hermaphrodite, la proportion relative des glandes tellement variable, que la séparation des sexes peut en être la conséquence, et ne pouvons-nous, rapprochant cette observation de celle du Cardium serratum, en faire une application à l'Huître(3)?

Il faut avoir l'altention éveillée sur tous ces faits pour n'être pas entrainé vers des opinions en partic vraies, mais qui, trop absolues, deviennent fausses. Ainsi quand, dans $ı$ individu plus femelle que mâle, les oufs masquent et eachent le testicule, il faut avoir du courage et ne pas abandonner trop vite les recherches pour trouver des spermatozö̈des; quelquefois ce n'était qu'après une journée entière employée à examiner tous les points de la glande que jarrivais à trouver des filaments, et que je considérais l'individu non plus comme femelle, mais comme hermaphrodite.

Le nombre des individus que j'ai examinés s'élève tout au plus ì

(1) Ann. des sc.nal., $3^{\ominus}$ série, 1853, 1. XX.

(2) Loc. cit., p. 307.

(3) Ann. des sc, nat., 3e série, + XX, p. 337. 
deux cents ; je n'ai pas été à un millier comme M. Davaine : car, pour rechercher convenablement dans tous les pointsde la glande, et décider si telle ou telle vésicule est ou n'est pas un œuf, il faut beaucoup de temps, et il ne suffit pas de piquer au hasard ou d'examiner rapidement une large tranche de la glande. Mes observations, quoique moins étendues, ont établi ma convietion; car toujours clles ont porté suir des Huìtres qui venaient d'être pêehées le plus souvent par moi, quelquefois par les plongeurs de Mahon, dont le port se prête si admirablement à ees sortes de recherches, par la limpidité et la tranquillité vraiment merveilleuses de ses eaux.

On comprendra maintenant pourquoi les auteurs, tombant sur tels ou tels individus, ont nié l'hermaphrodisme ou l'unisexualité.

\section{III.}

Considérations générales.

Les nombreux détails qui précèdent ont mis hors de doute ce fait, que l'appareil de la génération est d'une grande simplicité dans tout le groupe des Acéphales lamellibranches; on le voit, pour ainsi dire, réluit à la partic productrice des éléments sexuels qui, dans quelques eas, tombent dans les mêmes conduits excréteurs devenus communs aux deux sexes. Aussi la comparaison entre les organes mâles et les organes femelles peut-elle être établie avec facilité et évidence dans tout le groupe.

Il serait intéressant de rechercher les analogies qui existent entre les organes des sexes chez les mollusques en général: mais les observations manquent souvent, et bien qu'il existe des monographies anatomiques détaillées, on ne doit eependant établir la comparaison qu'avec beaucoup de réserve. Il m'a paru que dans les Aplisies, les Limaces, les Oseabrions, les Patelles, les Fissurelles, l'Haliotide et quelques autres Gastéropodes de différents groupes, les oufs et les spermatozoïdes avaient une origine et un développement qui pouvaient se rapprocher, à beaucoup d'égards, le ceux qui ont été indiqués ici; mais aussique sur beaucoup d'autres points de la ruestion, des recherches devraient être entreprises daus un hut spécial, et qu’alors seulement une ćtude comparative 
pourrait ètre faite avec quelque intérêt, et conduire à des résultats utiles.

Depuis que les progrès de l'histologie et de l'anatomie mieroscopique, appuyés sur les notions de physiologic générale, sont venus montrer, dans toutes les sécrétions de l'économie, une analogie très grande, puisque toujours, quel qu'en soit le but, la nature du produit diffère seule ou a établi des comparaisons fréquemment entre les organes mìles et femelles, et souvent on a été conduit ì des résultals philosophiques heureux. Pour le groupe qui vient de nous occuper, aucun rapprochement de ce genre n'ayant ćté fait, je saisirai l'oceasion pour établir un parallèle qui semble facile, et dont l'ensemble résumera les faits qui ont été consignés précédemment.

D’abord, pour rencontrer une différence appréciable, il faut remonter jusqu'aux parties profondes de l'apparcil; tout l'ensemble des vaisseaux excréteurs offre une analogie telle, dans le mâle et la femelle, que son étude seule ne pourrait faire reconnaître les sexes. Que l'attention se porle sur l'orifice, sur le mode de ramification des canaux, sur leur structure, partout on voit des caraclères qui s'appliquent, sans aucune réserve, aussi bien au mâle qu'à la femelle. La glande dont nous avons signalé les rapports avec l'orifice génital, qu'on la considère ou non comme une annexe, ne présente pas la plus légère différence dans les deux sexes.

linsi, pour trouver des différences appréciables, il faut, abandonnant toute la partie accessoire de l'appareil génital, remonter jusqu'ì celle qui sécrète, jusqu'ì celle rqui seule caractérise l'organe, et dans laquelle nous voyons se former d'me part un œuf, de l'autre un spermatozö̈de. Nous n'avons douc à nous occuper que de rechercher si les acini miles sont semblables aux acini femelles, si les éléments qui dans l'un séerètent le filament spermatique sont les analogues de ceux rui dans l'autre produisent l'ceuf. On le voit, la question du parallèle se réduit presque à celle-ci : les sécrétions du sperme et de l'ouf proviennent-elles l'une et l'autre d'un même tissu, et la théorie de la sécrétion qui nous montre cette fonetion eomme une chute ef une reproduction incessante de ecl. lules, se trouve-t-elle applicable iei? En restreignant de plus en 
plus les limites du parallèle, on arrive ì se demander quelle est la cellule des acini mâles qui correspond à la cellule des acini femelles.

Quand on a examiné, à un grossissement suffisant, les deux extrémités arrondies des culs-de-sac sécréteurs, mâles et femelles, avant qu'ils soient gorgés d'une trop grande quantité de leurs produits, avant, en un mot, qu'ils soient complétement arrivés à leur maturité, on voit que leur substance placée en dedans de la membrane d'enveloppe est, dans l'un et l'autre cas, purement cellulaire. La seule différence appréciable entre les cellules parait être dans le volume; de plus, la multiplication des premières semble être endogène, ce qui n'a pas été observé pour les femelles.

Dans chacune des cellules on a vu se développer, là un œul, ici un filament spermatique. Les différences deviennent alors très appréciables, et ces deux éléments constituent les produits définitifs de la sécrétion. Il ne paraît pas possible de pousser plus loin la comparaison; charque glande a sa spécialité, et son produit jouit et doit jouir du même privilége. L'ouf et le spermatozoïde ont leur but chacun bien marqué, de là ces différences que l'on observe dans la composition des parties qui les produisent. Ceciétabli, on comprend où s'arrête, à nos yeux, le parallèle : il doit finir lì où des produits nouveaux ont des destinations distinctes; aussi ne suivrons-nous pas les auteurs dans les spéculations plus ou moins ingénieuses qui les ont poussés à reconnaitre des analogies entre des choses qui nous paraissent par trop différentes pour pouvoir être comparées. Pour nous le parallèle peut être établi dans l'ensemble de la composition de la glande jusqu'à son produit, devant lequel il faut s'arrêter. Alors les acini mîles paraissent eomposés, de même que les acini femelles, d'une couche de cellules ou de corpuseules, comme on voudra les appeler, destinés à secréter les produits. Les éléments de cette conche qui tapissent le cul-de-sac sécrétcur sont beaucoup moins volumineux dans les mâles que dans les femelles, parce que dans les uns le produit sera infiniment plus petit que dans les autres. Il est naturel de voir dans la différence du produit la rause de la différence des éléments qui le fournissent : aussi nous parait-il y avoir unc analogic complète cntre un cul- 
de-sae sécréteur du sperme, et un cul-de-sae sécréteur des wufs; et cela est si vrai, qu'avant la gestation, e'est-à-dire bien avant que les éléments se dessinent, que l'cuf et le spermatozoïde soient formés, on éprouve une grande difficulté à pouvoir reconnaître le sexe des Acéphales : e'est que la glande est purement cellulaire, et que les cellules qui la composent n'ont pas encore pris le développement qui les caractérisera plus tard.

Du reste on retrouve, dans les deux cas, ume explication de la sécrétion complétement en rapport avec la théorie générale. Lorsque la cellule qui a produit un germe ou un filament est arrivée à maturité, elle se rompt ou tombe dans la cavité du cul-de-sac; alor's elle est emportée par les courants, elle est rejetée et excrétée. Aussi nous parait-il que dans la production du sperme et des aufs, on ne doit voir qu'une succession de chutes et de reproduetions de cellules, comme dans les autres glandes.

Si l'on s'arrête dans la comparaison, au point qui nous a semblé devoir être celui où toutes les analogies, les ressemblances et les caractères comparables cessent d'exister, on aura peine ì comprendre l'opinion de M. Van Beneden, qui a cru pouvoir démontrer' l'analogie du spermatozoïde avee la cellule vitelline. Dans une gangue quelconque, dit cet auteur (1), il se forme des oufs, de même que dans une partie organique toute semblable il se formera des spermatozoïdes. " Il y a un ouf, pour la forme au moins, dans " les deux cas. Au milicu de cet ouf, cellule ou vésicule, on voit " apparaître ensuite un grand nombre de petites cellules qui, par " leur réunion, forment le vitellus pour le produit femelle, et qui, " dans le produit mîle, se répandent au dehors séparément, en dé» chirant la vésicule qui les contenait. C'est un spermatocyste dans „ ce dernier cas, un vitello ou embryocyste dans le premier. „ Suivent ensuite des explieations qui indiquent que c'est par l'allongement de ces cellules vitellines que le zoosperme se constitue et prend sa forme distincte.

Pour pouvoir admettre cette opinion, il faudrait d'abord avoir reconnu dans l'(euf cette formation de cellules ; et ensuite avoir

(1) Van Beneden, Bull. de l'Acad. des sc, de Bruxelles, t. XI, 1844: Hermuphrodisme de l'Anodonte, p. 382-383. 
rencontré d'une manière constante la cellule mère de l'élément qui produira le filament spermatique. D'ailleurs, en admettant ces idées, que deviennent la vésicule germinative et la tache? On doit aussi en tenir compte. 1I. Van Beneden parait s'être occupé de rechercher, dans le spermatocyste, les vésicules de Purkinje et de Wagner; mais il n'a pu découvrir rien d'assez net pour avoir une certitude (1).

Du reste, il ne faut pas oublier que ce parallèle de l'organe mâle et de l'organe femelle est fait sur l'Anodonte, animal considéré comme hermaphrodite par M. Van Beneden, tandis que Leuwenlıoeck (2) et Siebold (3) le déclarent à sexes séparés; que si ces derniers auteurs sont dans le vrai, on peut s'expliquer toutes les analogies trouvées par le savant belge.

Si l'on considère le nombre des espèces qui ont été étudiées, on remarque que les cas d'hermaphrodisme sont, on peut le dire, bien peu multipliés. Trois genres seulement ont présenté les deux glandes portées par le même individu, et encore, dans l'un d'eux, une espèce rentre dans la catégorie la plus nombreuse, dans celle où les sexes sont séparés; le quatrième exemple est une exception đans un genre ì sexe séparé. N'est-on pas conduit, même en tenant compte des exemples signalés par Siebold, ete., à croire que l'hermaphrodisme est l'exception, tandis que la séparation des sexes est la condition habituelle. Cette opinion se trouve encore confirmée par les observations qui montrent dans l'Huître que plus l'un des sexes prédomine, plus la régularité semble renaitre. On croirait là, que plus la tendance à l'état normal reparait, plus la disposition tend à devenir simple et semblable à celle qui s'observe dans les cas oì la condition exceptionnelle n'existe pas. Et je ne puis m'empêcher de dire ici quelle impression faisait sur moi une espèce nouvelle que j’allais étudier. Souvent, à l'aspect de la glande (après avoir aequis une certaine habitude), j'ai pu reconnaître si j’avais affaire à une espèce à sexes séparés, et, redoutant des idces à priori, j’ai multiplié mes recherches dans la crainte de faire des erreurs; il ne m'est arrivé d'avoir à modifier mon opinion que

(1) Loc. cit., p. 383

(2) Leuwenhoeck, Arcana naturæe detecta, lettre 59

(3) Siebold, Anat. comp., t. I, p. 286. 
pour la Pandora rostrata et le Cardium serratum. Linsi, pour ne citer qu'un exemple, combien de Venus ai -je examinées ì Mahou, à Barcelone, à Marseille, ì Martigues, ì Cette, à Rochefort, à lì Rochelle, en Bretagne; jamais un exemple, même douteux, ne s'est présenté. Mais, dans cette espèce, quelle régularité surtout dans le testicule que l'on reconnaît, à l'œil nu ou avec la loupe, ì la forme toute particulière de ses culs-de-sac sécréteurs. Dans un autre exemple, dans la Donax anatinum, je n'ai rencontré qu'un seul individu en gestation, e'était un mâle. J'ai la conviction que, dans cette espèce, les sexes sont séparés; il sera curieux de voir si plus tard cet à priori sera confirmé ou reconnu faux.

Ainsi, l'opinion ancienue de Poli, reproduite dans presque tous les ouvrages, celle plus moderne de MII. Garner et Deshayes, sur un hermaphrodisme général, doit être complétement rejetée et n’être plus considérée que comme applicable à quelques cas relativement assez rares. Ce résultat, indiqué comme absolu par R. Owen, sans être basé sur des faits nombreux et trop généralisés, reconnu par von Siebold dans plusieurs espèces, conduit naturellement ì se demander comment s'effectue la fécondation?

Il est un fait qui frappe quand on s'oceupe des sexes, eu égard aux espèces en général. On a vu deux genres, l'un hermaphrodite (Pecten), l'autre unisexué (Cardium), présenter chacun une espèce où les conditions sexuelles dérogent à la règle générale. $A$ la première vue, sans fairc même une étude approfondie des caractères, on voit quelque chose de cette anomalie des sexes se traduire dans l'apparence des animaux. Ainsi on ne saurait nier que le Peigne. bigarré n'ait un aspect générique un peu différent des autres Peignes hermaphrodites. De même dans tout l'ensemble du Cardium serratum, on découvre quelque chose de spécial qui indique des différences plus grandes entre l'espèce hermaphrodite et les espèces à sexes séparés, que celles qui distinguent ces dernières entre elles.

Les études au point de vue de la classification n'ont pas été assez complètes pour qu'il soit permis de penser que des geures nouveaux seront utilement établis d'après ces différences. Mais enfin cette obscrvation laisse une impression dont il est difficile de se 
défendre, et qui pourrait peut-être plus tard conduire à une application.

Il ne faut pas se dissimuler toutefois que l'observation de 11. Humbert sur le Peigne glabre de Cette fait perdre un peu de sa valeur aux caractères tirés de l'hermaphrodisme et de la séparation des sexes. Il est évident, en effet, que si une espèce peut se présenter à l'état hermaphrodite ou à l'état unisexué, la réunion et la séparation des sexes a, par cela même, une importance bien moindre.

C'est pour n'avoir pas connu des faits de ce genre que M. Davaine a été conduit pour les Huîtres à admettre une opinion que nous avons eu l'occasion de critiquer déjì. Cet auteur se denande, après avoir reconnu les diverses apparences sous lesquelles se présente l'Huître, sila séparation des sexes est la loi, et l'hermaphrodisme l'exception, ou réeiproquement. " Admettre que les Huitres » sont indifféremment hermaphrodites ou ì sexes séparés, c'était ") admettre une condition encore ineonnue dans le règne animal. „ Ces considérations nous portèrent à penser que les diverses appa"rences qu'avait offertes à notre examen l'organe reproducteur " des Huîtres n'étaient que des phases de l'état le plus compliqué de "l'hermaphrodisme (1)... "

On voit comment la connaissance des faits comparatifs faisant défaut, l'auteur arrive, pour ne pas admettre une condition inconnue dans le règne animal, et qui existe cependant, à reconnaitre des phases dont il cherehe à démontrer la succession. Cette condition, qui, pour nous, n'est plus inconnue, vient à l'appui de l'opinion opposée à celle de M. Davaine.

La succession des périodes d'activité des deux glandes ne s'accorde pas avee ce que l'on observe dans les autres espèces hermaphrodites, où jamais le testicule n'a paru plus précocement développé que l'ovaire. Dans les Pandores, les Peignes, les Bucardes hermaphrodites, les deux glandes marchent parallèlement dans leur évolution. L'opinion qui consiste à admettre que les différentes apparences sexuelles de l'Huitre sont dues à un développement plus ou moins considérable de l'une des glandes, se trouve appuyée sur

(1) Loc. cit., p. 307 , alinéa $u$. 
des faits complaratifs qui nous montrent la mème espèce pouvant passer par des étals intermédiaires de l'hermaphrodisme à l'uniscxualité. Elle est d'ailleurs en rapport arce l'importance moindre qu'il semble falloir accorder à la réunion ou à la séparation des sexes. Et c'est évidemment la valeur absolue que M. Davaine altache à cette séparation, qui l'a conduit à chercher et à trouver l'explication motivant son opinion.

La rencontre de l'œuf et du sperme pent avoir lieu en deux points : dans l'animal et hors de lui.

Dans le premier cas, qui se rapporte à l'hermaphrodisme, l'opinion de Cuvier est applicable, quand il dit (1) que « ces animaux se suffisent et se fécondent cux-mêmes. v Mais il ỹ a une distinction à ćtablir en raison des deux espèces d'hermaphrodisme que l'on a vues exister. Dans les Iuitres, en effet, la question de savoir si les acini sécrétaient à la fois l'œuf et le spermatozoïde n'a pu être résolue, mais il est resté démontré que les acini mâles et les acini femelles étaient au moins côte à côte et insérés aux extrémités des mêmes conduits. Dans le Cardium serratum, le doute qui existe à l'égard de l'Huître a complétement disparu. De là un contact mmédiat des éléments presque au noment de leur sécrétion, et de leur départ desacini sécréteurs. Aussi l'œufde l'Huitre est-il presque toujours fécondé, et les premières phases de son développenent sont-elles difficiles à observer. Nous voyons done cheminer, dans les cas d'hermaphrodisme avec mélange des glandes, le fluide fécondant du mâle à côté de l'ovule de la femolle, depuis le point où ils ont été l'un et l'autre produits.

Dans le second cas, pour l'un des genres, pour les Peignes, les éléments peuvent et doivent même se rencontrer daus le tube excréteur, mais beaucoụ moins vite que dans l'Huître; on doit même comprendre que, lorsque la ponte s'effectue avec rapidité, il peut se faire que des oufs sortent sans aroir été influencés par la liqueur mîle : si cela arrive, on voit, d'un autre côté, que la fécondation se fait au moins dans la cavité de la glande de Bojanus, oì tombent souvent des produits de la génération.

Mais comment se rendre compte de la fécondation daus la ma-

(1) Règne animal. 
jeure partic des cas, e'est-ì-dire quand les sexes sont séparés? Faut-il, avec Méry, croire à une sorte d'accouplement? Il suffit de voir comment vivent les Lithodomes, les Pholades, etc., pour rejeter tout de suite une pareille supposition qui pourrait tout au plus s'appliquer à quelques espèces vivant libres de toute adhérence, mais qui ne pourrait plus nous expliquer la fécondation des Anomies, des Cames, des Spondyles, ctc.

Voudrait-on voir un rapprochement des tubes formès par le manteau entre des individus voisins, comme le sont dans une même pierre les Pétricoles, les Gastrochènes, dans un même fond sablonneux les Bucardes, les Clovisses, les Solens? Mais alors on devrait considérer que les individus éloignés les uns des autres resteraient inféconds. Ce serait lì une supposition qu'aucune observation ne démontrerait.

Il faut done avoir recours à une autre explication. On sait de 'juclle agilité, de quelle mobilité, et surtout de quelle résistance vitale jouissent les spermatozoïles. M. de Quatrefages a vu ceux du Taret virre très longtemps, et, pour la plupart des espèces dont il est ici question, j’ai répété la même observation. N'est-il pas évident que ees propriétés sont en rapport avec les conditions physiques de leur action. Dans les animaux supérieurs, un peu d'eau, un léger refroidissement, font cesser immédiatement tous les mouvements. Sorti des vésicules séminales du mâle ou dı vagin de la lemelle, le sperme perd ses proprićtés fécondantes.

Tandis qu’ici l'eau, celle du moins des milieux dans lesquels vit l'animal, parait animer les filaments pris dans la glande; e'est que l'action doit s'opérer à distance, et que le spermatozoïde est obligé d'aller à la rencontre de l'œuf. Aussi peut-on comparer la fécondation des Acéphales lamellibranches diö̈ques au phénomène si bien ćludié par Linné dans les plantes qu’il avait désignées par ce nom.

Le mâle lance sa semence, elle va féconder les aufs pondus flottant daus l'eau, ou se trouvant dans le manteau entre les branchies de la femelle, comme cela se voit pour un grand nombre d'espreces l'eau entraine le sperme que les courants et les mouvements ciliaires de la surface interne du mantean font pénétrer 
justu'aux wuls. Qne l'on se figure une pierre criblée par les trous des Gastrochènes, des Lithorlomes, ete., n’est-il pas évident que les produits rejetés par le tube postérieur des uns sera absorbé par le tube antérieur des autres. Les courants nécessaires aux besoins de l'alimentation et de la respiration sont très forts ; on les observe avee la plus grande facilité. Les Pholades, les Lutraires, lorsque la couche de liquide qui couvre l'extrémité de leurs siphons est pen épaisse, lancent un jet qui dépasse un peu le niveau de l'eau. Ces courants n'ont rien qui se lie à la génération; ils ont pour but d'autres fonctions, mais néanmoins ils doivent coneourir à l'accomplissement de la fécondation. Ainsi, le sperme lancé par une Gastrochène, entrainé par le courant le plus voisin, tombe dans le manteau d'un individu soit mâle, soit femelle. Ce que je dis des Gastrochènes, je le dirais des Lithodomes enfermés dans la pierre, des Bucardes, des Lucines, des Lavignors, des Solens, enfouis dans le sable, ete. Les mouvements de l'eau facilitent ou éloignent les chances de la fécondation ; aussi faut-il reconnaitre que cette première période de la fonction de reproduction est complétement abandonnée au hasard. La ressemblance avec ee qui se passe dans les plantes diö̈ques est frappante. La plante mâle laisse tomber son pollen, c'est le vent rui se charge de le porter à la rencontre d'un pied femelle. Dans un cas c'est l'eau qui sert au transport, dans l'autre c'est l'air ; l'un a ses courants, l'autre ses vents; que si les mouvements de ces milieux sont contraires, l'infécondité en est la conséquence. La comparaison peut être soutenue, mais cependant sans que pour les Acéphales la fécondation puisse avoir lieu à des distances comparables ì celles que l'on a citées dans quelques cas pour les végétaux.

Ce mode d'influence du mâle sur la femelle avait été admis, dès 1825, par Prévost, dont ¡’ai rapporté les expériences si bien conduites : il avait vu les Unio mâles féconder les femelles sans qu’il y eût rapprochement. Cette manière de comprendre le premier phénomène de la reproduction est certainement l'expression de la vérité, car on voit les espèces où la fécondation est la mieux assurée se reproduire avec le plus de facilité. Ainsi quelle n'est pas l'étomante fécondité de l'Huitre? Elle forme des banes que les pêcheurs sont sans cesse orcupés à détruire, el qui erpentant se. 
reforment toujours ; mais pour cette espèce la chance du hasard de la fécondation n'existe pas. Les Moules se reproduisent aussi avec une facilité remarquable, bien qu'elles aient les sexes séparés. Cela tient à ce qu'elles se réunissent, se groupent, ce qui, diminuant les ehances d'inaction du sperme, augmente le nombre des reufs fécondés. Plus les espèces sont rapprochées, plus nous les voyons se multiplier.

Quand je cherchais dans le port de Mahon des Chama gryphoides qui vivent fixés aux rochers, j'avais fait la remarque suivante : si, après quelques recherches, je ne trouvais qu'un individu isolé, je ne devais pas m'attendre à en rencontrer beaucoup, je devais changer de place; si, an contraire, trois, quatre individus se présentaient assez rapprochés, habituellement ma pêche était bomne. N'y a-t-il pas dans ce fait quelque chose qui montre que le sperme, porté au hasird, est souvent perdu quand les femelles sont trop éloignées, et que celles-ci placées à une grande distance, isolées el fixées à des rors, pondent des oufs qui restent inféconds, jusqu'ì ce que les flots apportent quelque larve mâle daus leur voisinage, dont le driveloppement ultérieur répondra aux besoins de la fécondation.

On sait que tous les observateurs ne sont pas d'accord sur la question de savoir lequel du liquide ou du filament est la partie active dusperme. Les spermatozoïdes ne servent-ils par leurs mouvements qu’à transporter la liqueur sur l'œuf, ou bien sont-ils récllement l'élément modificateur? C'est ee qu’il est bien difficile de pouvoir clire. Toutefois l'observation montre que les mouvements des spermatozö̈des ef leur présence sont deux conditions absolument nécessaires pour que le sperme puisse agir sur les neufs. Si l'on rapproche ee fait des conditions dans lesquelles on vient de voir s'accomplir la fécondation des Acéphales diö̈ques, on doit consenir que l'importance des spermatozoïles augmente eneore; (ar, après avoir traversé des couches d'eau assez étendues pour arriver jusqu’à l'oulf, les filaments doivent être débarrassés de la plus grande partie du liquide spermatique qui les baignait, et l'on ne peut guire se refuser i admellre que seuls ils soient les véritables agents fécondiunts. 
Lne deruic̀re considération est eelle qui loude ì l'époyue de l'amnée pendant laquelle les Lamellibranches se reproduisent. En général, durant l'hiver, les glandes génitales restent inactires, ou du moins elles ne font que se préparer ì entrer en activité, et alors leur structure n'indique point le sexe de l'individu. La chaleur fait développer, comme, du reste, pour la plupart des animaux, les organes de la génération; aussi éest pendanl l'été que les recherehes sur la reproduction des Molluspues qui uous occupent sont les plus fructueuses. On roit, du reste, que la gestation des $A$ céphales varie avec les époques et avec les localités. Poli a donné, à cet égard, des indications très justes dius son magnifique ouvrage que nous arons eu si souvent l'oceasion de citer : " Il importe de savoir, dit-il, que tous ces animax ne pon»dent pas à la mème époque de l'annće : Qucedam vere ineunte, "quadam cestate, quadlam dum autumno atque hyeme (1). "D'après lui, les Myes, les Solens, fraient au commencement du printemps; la Pholade, la Came, la Vénus, la Donace, l’Anomie, la Telline, la Mactre, dans l'été; et la Moule, au mois d'octobre jusqu'en décembre. En ce dernier point, Polin'est pas d'accord avec Baster 2 , qui a vu la même espèce, en Hollande, frayer au mois de mars ef d'avril. L'auteur italien rapporte que les pêcheurs de Tarente n'ont jannais observé le frai de la Houle dans ees mois. On comprend que la différence de la température entre les deux pays où observaient les deux auteurs est assez grande pour expliquer la divergence de leurs opinions. Ce qu'il y a de certain, e'est que, sur les côtes de l'Océan en France, Boulogne, Calais, la Rochelle et la Tremblade, les organes génitaux des Moules sont en état de gestation aux mois d'avril et de mai; on croit même que dans cette période liı Iloule comestible devient un aliment nuisible, peut-être dangereux (3). Les individus rares que j’ai pu avoir à Vahon étaient au mois de juillet complétement privés d'ceut on de sperme. J’ai

(1) Loc. cit., t. I, p. 68 .

(2) Loc. cil.

(3) La question des empoisonnements par les Houles est encore mal connue, et des recherches à la fois médicales et scientifiques doivent être entreprises par des hommes spéciaux. 
olsservé la même chose aux Martigues, dans l'étang de Berre, pris de Marseille, pendant le mois de septembre. Il est peu probable: que, dans cette dernière localité, le développenent des orufs pit commencer, alors que la température baissait déja beancoup; mais ce qui est vrai pour une localité doit épronver des changements pour une autre.

Depuis le commencement de mai jusqu’à la fin de septembre, à Barcelone, à Palma, ì Mahon, à Marseille, atux Martignes, à Cette, j’ii trouvé des Vénus et des Peignes higarrés et glabres dans um état parfait de gestation : les reufs el les spermatozoüles abondaient; mais dans la série des mois qui viennent d'être indiqués, et qui correspond à l'ordre suivant lequel les localités ont été explorées, le nombre des individus ayant des glandes turogides et très gonflées par les germes ou le sperme allait en diminuant. Les Vénus se trouvaient, à Cette, dans le même état que celui où je les ai trouvées à la Rochelle en avril 1854. Ce qu’on peut expliquer par un arrêt de la ponte, eausé par l'arrivée des froids.

J'ai trouvé, il Cette, dens les derniers jours de septembre, des luittes eneore en lait; mais les pêcheurs indiquent le mois de juillet comme le moment où cet état est le plus fréquent, et cela est vrai : il faut compler que le frai commence le 15 juin et finit le 15 aoùt. Les observations que j’ai pu faire encore cette année ì Saint-Jacut, sur les còtes de Bretagne, sont complétement d'aceord avec ce ru’indiquent les hommes ineessamment occupés à maniel les Huìtres qu’ils livrent an commerce.

J'ai étudié fréquemment, à Barcelone à la fin d'aoùt, et à Cette en septembre, la Donace (Donax anatinum), et je n'ai trouvé qu'un seul individu en élat de gestation. La Mactre (Mactra stultorum), dans la première localité et à la même époque, m’a paru aussi avoir pondu. Vers le courant de septembre, la T'elline (1) et la Psammohie (2) avaient pondı; leurs glandes étaient flétries. Dans l'été, 'oll Bretagne, j’ai rencontré la Mactre sans auf; ef je erois qu'ayrès ces deux observations faites sur des points aussi ćloignés,

(1) Tellina solidula.

(2) Psummobia vespertina. 
on peut conclure que la reproduction de cet Acéphale a lien an rommeneement de l'été ou dans le printemps.

I mon arrivée sur les côtes de la Bretagne, le 26 juillet 1854, je trouvai des Pholades d'une très grande taille dans un état parfait pourl'ćlude des organes dela génération. Les glandes, extrêmement développíes, oecupaient, pour ainsi dire, tout le corps ; elles étaient gorgées d'oufs ou de spermatozoüdes, suivant le sexe. Cet élat dur:a jusqu'au commencement de septembre, où je les examinai de nouveau, ayant pu, pendant la grande marée de la pleine lune du 8 , me procurer de nouveaux individus. Mais, ì la grande mer suivante, je trouvai les Pholades presque toutes sans arufs; il n'était pas douteux qu'elles n'eussent pondu pendant la morte mer, r'est-ì-dire dans une époque voisine du 15 et du 16.

Les Pandores, à cette éporque, étaient exactement dans les mêmes conditions, et leur ponte eut lieu aussi pendant la morte-cau du 16.

Voilà deux espèces, si l'on en juge par cette observation, qui sont bien plus tardives à se reproduire que les autres.

Dans la même localité, pendant une période de temps comprise entre les fins de juillet et de septembre, j’ai pu étudier fríquemment les Coques (Cardium rusticum et les Clovisses (Venus decussata), qui abondent dans les plages des Hébiens, et j’ai remarqué que leurs glandes génitales n'étaient point développées. Cependant à la même saison, ì Mahon et à Cette, toujours j'avais rencontré les organes génitaux en parfait état sur ces mêmes espèces.

De tous ces fails, il résulte évidenment que le moment de lis reproduction varie pour la même espèce avec la localité. Cela seul suffit, je crois, pour montrer comment Baster, qui observait daus les Pays-Bas, et Poli, qui faisait ses étules en Sicile, ont pu être en désaccord, bien que cependant l'un el l'autre fussent probablenent dans la vérité.

On peut, en résumé, admettre, je erois, avec le vieux proverbe qui nous dit que les huitres sont moins bonnes pendant les mois sans $r$ (c'est-i-dire quand elles fraient), que les Acéphales lamellibranches pondent et se reproduisent en général de la fin dı printemps au commencement de l'automne; que l'époutur ne peut ìtre fixće absolument pour aueune espice; que les con- 
ditions de température, tenant au climal, font varier beatcoup ce moment; que, de plus, il y a des différences individuelles yni expliquent comment une espèce présente des individus au terme de leur gestation un ou deux mois après l'époque la plus habiturlle.

Tous les auteurs qui ont étulié la reproduction des Acéphales ont été frappés de la prodigieuse fécondité de ces animaux. Il ne me semble guère possible de donner, conme ils l'ont fait, des chiffres qui expriment d'une manière satisfaisante le nombre des oufs pondus par un seul individu. Je dirai seulement, pourdonner une dée de la quantité d'ocufs qu'une Ifuitre de grande taille peut pondre, que j'ai recueilli sur le Pied-de-cheval de Mahon des larves qui ont formé une colonne de près de 3 centimètres $1 / 2$ de hauteur dans un tube de verre de 8 millimètres de diamètre. Les jeunes larves avaient environ $1 / 10^{\circ}$ de millimètredans leur grand diamètre. Le nombre de petils produits par une Huitre est donc immense.

Si l'on rapproche ec fait de l'époque où a lieu la gestation, on verra quel préjudice considérable la pêche pendant l'été porte à la reproduction des banes : car en enlevant une seule Huìtre remplie. de larves on détruil un nombre immense de jeunes individus ; landis que si l'on attend que les larves deviennent indépendantes, qu'elles aient abandonné le manteau de leur mère, on détruit bien encore beaucoup de jeunes Huîtres, mais un grand nombro chappent encore aux dragues et aux filets.

Je erois utile de domer la liste des espèees qui ont servi à mes observalions; j’y joindrai l'indication des localités on jo les ai rencontrées, et quelquefois les noms qu'elles y reçoivent.

Ostrae edulis et Hippopus, Huilre comestible et Pied-de-cheval, Lamk, 2e édit.,

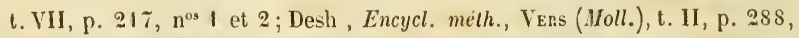
$\mathrm{n}^{\text {os }} \mid$ et 2. - Méditerranée, Océan.

Ostrea stentina, Huitre stentine, Payr., Cat. des Moll. de Corse, p. 81, n 154 , p 3, fig. 3; Lamk, 2e édit., t. VII, p. 236, $\mathrm{n}^{0} 50$. - Mahon.

Aхоми Epuppius, Anomie pelure d'oignon, Pélau à Saint-Jacut, Lamk, se édit., 1. VII, p. 273, n 1: Brug., Encycl. méth., t. I, p. 72, pl. clxx, fig. 6, 7, $n^{\circ}$ ö. - Mahon, Cette, la Rochelle, Bretagne. 
Pecten Jacob.eus, Coquille de Saint-Jacques, Lamk, 2 e édit., t. VII, p. 130 , $1^{\circ} 3$; Poli, Test., II, tab xxvı, fig. 1, 2. - Mahon, Cette, la Rochelle.

Pecten maxinus, Peigne à cótes rondes, Ricardiun en Brelagne, Lamk, t. VII, $2^{\mathrm{e}}$ édit., $\mathrm{n}^{\circ}$ 1; Desh., Encycl. méth., t. II, p. 270. - La Rochelle, SaintJacut-la-Mer.

Pecten varius, Peigne bigarre, Lamk, ib., p. 147, $1^{\circ} 47$; Desh., ib, p. 275, pl. 213, fg. 5; Forbes et Hanley, t. II, p. 273. - Mahon, Cette, la Roclielle, Rochefort.

Pecten glaber, Peigne glabre, Lamk, ib., p. 137, n² 20 ; Desh., Encycl. méth., t. II, p. 270. - Mahon, Cette.

Sposdyus G.eneropus, Pied-d'ane, Huitre vermeille, Ostia vermella (en mahonais), Lamk, t. VII, $2^{\mathrm{e}}$ édit., p. $184, \mathrm{n}^{\circ} 1$; Desh., Encycl. mélh., Vers, t. III, p. $978, \mathrm{n}^{\circ}$ 1. - Mahon.

Lisa squanosa, Lime commune, Lamk, t. VII, $2^{\mathrm{e}}$ édit., p. $115, \mathrm{n}^{\circ} 2$; Desh., Encycl. méth., Vers, t. II, p. 345, no 3. - Mahon.

Pixsa xobilis, Jambonneau, Pinne hérissé, Nacre, Lamk, t. VII, $2^{\mathrm{e}}$ édit., p 62, n ${ }^{\circ} 5$; Desh., Encycl. méth., t. III, p. 768, n². - Mahon, Palma (aux Isletas).

Arca Noe, Arche de Noé, Pied-de-chevreau, Pe de cabril (en mahonais), Lamk, t. VI, 2e édit., p. $461, \mathrm{n}^{\circ} 3$, Encycl. méth.; Brug., t. I, p. 97, $\mathrm{n}^{\mathrm{0}} 2$; Poli, Test., II, tab. xxıv, fig. 1 et 2, p. 138, t. II. - Mahon, Palma (las Isletas).

Pectuxculus pilosus, Pétoncle fammulé, Coquilles anglaises, Scupiñas inglesias (en mahonais), Lamk, $2^{e}$ édit., t. VI, p. 488, n² 2 ; Desh., Encycl. mélh., t. III, p. 741, n² ; Poli, Test., t. II, tab. xxv, fig. 17, 18. - Mahon, Barcelone.

Anodonta cygneA, Anodonle des cygnes, Lamk, t. VI, $2^{\mathrm{e}}$ édit., p. $564, \mathrm{n}^{\circ} 1$. - Versailles, les étangs de Trappes.

Uxıo littoralıs, Mulette littorale, Lamk, t. VI, 2e édit., p. $538, \mathrm{n}^{\circ} 20$. — La Garonne (à Langon).

Unı pictonum, Mulette des peintres, Lamk, ib., p. 5\$1, n 32 ; Desh., Encycl. méth., p. 586, n² 20, fig. 1, pl. 248.-La Seine, près de Paris, à Bougival.

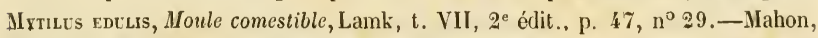
Palma, Marseille, les Martigues, Cette, la Rochelle, les côtes de la Nanche, en Normandie, en Bretagne.

Modjola Lithophaga, le Lithodome, la Datte, la Dattile (en mahonais), Lamk,

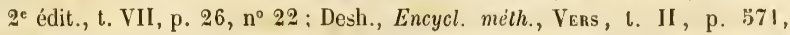
$\mathrm{n}^{\circ}$ 41; Genre Lithodomus de Cuvier, Règne animal, II, p. 471; Lithodomus lithophagus, Payr., Cat., p. 68, n 132. - Mahon.

Cham gryphoides, Came gryphoïde, Lamk, t. VI, $2^{\mathrm{e}}$ édit., p. $581, \mathrm{n}^{\mathrm{n}} 3$; Eucyel. méth., pl. 197, fig. $2 u, b, c$; Poli, Test., Il, tab. xxul, fig. 3. - Mahon.

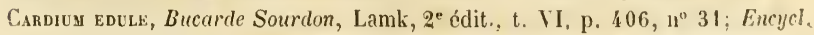
méth., Brug, n 13, t I, Vers (Ioll.). - Rochefort, la Manche. 
Cardium rusticum, Bucarde rustique, Lamk, ib., p. 405, nº 30 ; Brug., Encycl. méth., t. I, p. 222, no 15 . - Mahon, Cette, Rochefort, les Hébiens.

Cardium tuberculatum, $B$. tuberculée, Lamk, ib., p. 397, n 16 ; Brug., Encycl. méth., p. 229, n'12. - Cette.

Cardium cillare, B. frangée, de Bruguière, Encycl. méth.,p. $218, n^{\circ} 11$. Lamarck en fait son espèce echinalum différente de celle qu'il désigne par le nom de ciliare, Lamk, ib., p. 396, n 12. - Cette.

Cardium serratum, B. dentelée, Brug., Encycl. méth., t. I, p. 329, n$^{\circ} 37$; Lamarck, t. VI, p. 401, $n^{\circ} 35$; Pennant, C. lavigalum; Forbes et Hanley, C. norvegicum, t. II, p. $35, \mathrm{pl}$. xxxı, fig. 1 et 2. - Océan européen, d'après Lamarck et Bruguière; les Hébiens.

Cardium lavigatcm, B. lisse, Brug., Encycl. méth., t. I, p. 231, n 30 ; Lamk, t. VI, p. 403, n 26. - Des Antilles, d'après Bruguière.

Cardita sulcata, Cardile cannelée, Lamk, t. VI, p. 425, n$^{\circ} 1$; Brug., Encycl. méth., VERs, t. I, p. $405, \mathrm{n}^{\circ} 3$. - Mahon.

Lucina lactea, Lucine lactée, Lamk, t. VI, p. $228, \mathrm{n}^{\circ} 12$; Encycl. méth, t. I, p. $374, \mathrm{n}^{\circ} 9$; Reeve, t VI, 1849 à 1851 , sp. 41, pl. virt, tig. 41. - Cette, étang de Thau.

Donax anatinum, Donace des canards, Lamk, 2e édit., t. VI, p. $249, \mathrm{n}^{\circ} 26$; Desh., Encycl. méth., Vers, t. II, p. 99, n 17. - Barcelone, Cette.

Tellixa solidula, Telline, Desh., Encycl. mélh, t. III, p. 1919, n* 28. Saint-Jacut-la-Mer (les Hébiens).

Psammobia vespertina, Psammobie, Desh., Encycl. mélh., t. III, p. 851, nº 1 ; Lamk., $2^{\circ}$ édit., t. VI, p. 173, n 3 . - Saint-Jacut-la-Mer (les Hébiens). Trigonella piperata, Desh., Expl. de l'Algérie, Moll., p. 509; Lavignon, de Cuvier; Lutraria piperata et compressa, de Lamk, t. VI, 2 e édit., p. 91 et 92 , $\mathrm{n}^{\text {os }} 4$ et $5 ;$ T. plana, de Dacosta, Brit. conch., p. 200 , pl. xıI, fig. 1.- La Rochelle, Rochefort, les Hébiens.

Venus decussata, Vénus croisée, la Clovisse des ports de la Méditerranée, Scupiñas lisas (des Mahonais), Lamk, t. VI, p. 356, n 46.-- La Méditerranée, dans toutes les localités.

Conbula striata, Preres et scupiñas gravudas (en mahonais), Desh., Expl. de l'Algérie, p. 231, $\mathrm{n}^{\circ}$ 1; Fleming, Brit. un., 2e édit., p. 425. - Mahon, Marseille, les Hćbiens.

IIactra stultorum, Mactre des sols, Lamk, p. 99, t. VI, 2e édit.; de Poli, Linné, etc. - Barcelone; les Hébiens, à la Colombière.

Mra arenaris, Mye des sables, Lamk, 2e édit., t. VI, p. 74, $\mathrm{n}^{n} \mathbf{2}$; Desh , Encycl. méth., Vers, 1. II, P. 592. - $\Lambda$ Rochefort.

Lutraria solexoldes, Lutraire soleniforme, Desh., Encycl. méth., t. II, p. 387 , $\mathrm{n}^{\circ} 1$; Lamarek, t. VI, p. 90, $\mathrm{n}^{\circ}$ 1.- A Saint-Jacut-la-Mer, spécialement sur la placre de la Colombière et des Piettes (les Hébiens).

Pandora rostrata, Pandore rostrée, Desh., Encycl. mélh., t III, p. $697, \mathrm{n}^{n}+$ 
Lamarck., t. VI, P. 145, n 1.- A Saint-Jacut-la-Mer, spécialement sur la plage de la Colombière (les Hébiens).

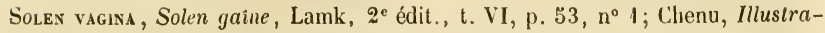
tions conchyliologiques; Poli, Test., t. I, pl. Lxxı, fig. 14; Desh., Encycl. méth., t. III, p. $959, \mathrm{n}^{n}$ 3. - La Rochelle.

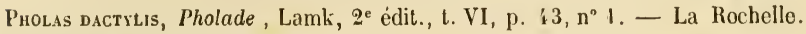

Gastrochexa debla, Desh., Expl. de l'Algérie, p. $34, \mathrm{n}^{\circ} 1$, ou Tarentina. Le même auteur l'avait indiquée dans l'Encycl. méth., sous le nom de Fistulana hyans, t. II, p. 141, $\mathrm{n}^{\circ} 3$; Sowerby, Genera of shells, Gastrochana modiolina, $\mathrm{n}^{\circ}$ 11; Lamk, $2^{\mathrm{e}}$ édit., t. VI, p. $49 ;$ G. modiolina, $\mathrm{n}^{\circ}$ 3. - II ahon.

Petricola Ruperella, Pélricole rupérelle, Lamk, $2^{\mathrm{e}}$ édit., t. VI, p. $159, \mathrm{n}^{\circ} 9$; Desh., Encycl. méth., p. 747, n² 3. C'est la Ruperella striala de Fleu riau de Bellevue. - Mahon.

\section{EXPLIGATION DES FIGURES.}

Nola. - Tous les dessins ont été pris à la chambre claire, et offrent des grossissements comparables; en ce sens que les mêmes éléinents ont été dessinés avec le même jeu de lentilles et à une même distance.

Les œufs ont été étudiés avec la lentille no 3 et l'oculaire n" 1 de M. Nachet; les spermatozoïdes avec le jeu $n^{\circ} 7$ et. l'oculaire $n^{\circ} 4$.

Les grossissements que j'indique ici sont plus faibles peut-être que ceux considérés comme appartenant aux combinaisons que je viens de citer. Je ferai observer que j'avais, pour la commodité des observations, raccourci beaucoup le tube du microscope, ce qui avait rapproché mon dessin, qui n'était guère éloigné de la chambre claire que de 18 centimètres.

Je fais ici cette observation, parce que les appréciations des pouvoirs amplifiants varient si souvent, même à l'insu de l'observateur qui hausse un peu plus, un peu moins, le papier sur lequel il dessine, que je crois qu'il serait utile que les micrographes s'entendissent enfin sur la longueur du tube du microscope, et la distance à laquelle on dessine et l'on mesure, afin d'avoir des résultats, je ne dis pas absolus, mais au moins comparables.

\section{PLANCHE 5.}

Fig. 1. Pinna nobilis (Nacre), demi-grandeur, vue de face, en dessous, pour montrer l'ovaire $(0)$ et ses rapports avec le foie $(f)$. Le sac de Bojanus $(r)$ et ses orifices $(c)$, qui sont aussi ceux des organes de la génération.

Fig. 2. Une portion du même vue par la face dorsale. L'ovaire $(0)$, le foie $(I)$, le sai; de Bojanus $(r)$, les muscles du byssus $(m)$, la gouttière intestinale formće par l'ovaire $(i)$.

Fig. 3. OEufs du mème à différents degrés de développement. Une résicule 
germinative isolée et entourée encore de granules vitellins. Grossissement, 170 diamètres.

Fig. 4. Coupe de l'ovaire du même, pour montrer les conduits excréteurs.

Fig. 5. OEufs non murs déformés par l'endosmose, de la Gastrochcena tarentina. Grossissement, 170 diamètres.

Fig. 6. OEuf mûr du même, non déformé. Grossissement, 170 diamètres.

Fig. 7. OEufs de la Corbula striata entourés de granulations. Cette figure, analogue à celle que donnent les auteurs, ne peut rien démontrer pour la structure de l'ovaire.

PLANCHE 6.

Fig. 1. Portion de glande femelle et de foie du Pecten varius, grossie, montrant ( très nettement les acini groupés en lobules primitifs. On voit dans le milieu du tube excréteur une teinte brunâtre due an produit de la sécrétion.

Fig. 2. Face droite; extérieur du manteau de la Mytilus edulis, montrant les canaux excréteurs se rendant aux lobules secondaires et primitifs en partant du point $b$, où le conduit est unique.

Fig. $2^{a}$. Spermatozoïdes de la même. Grossissement, 500 diamètres.

Fig. 3. Portion des acini de l'Arca Noe, pour montrer les capsules des œufs continues avec la substance même du tube sécréteur. Grossissement, 170 dia. mètres.

Fig. 4. OEuf du même: la capsule rompue, vide et plissée. Grossissement. 170 diamètres.

Fig. 5. OEuf du Pectunculus pilosus. Grossissement, 170 diamètres. Les capsules, en se rapprochant, semblent faire des aréoles qui logent chaque œuf.

Fig. 6. Trois culs-de-sac sécréteurs du Lavignon (Trigonella piperata). Les œufs, pressés les uns contre les autres, paraissent polyédriques. Cette appareuce est la plus fréquente dans tous les ovaires mûrs.

Fig. 7. OEuf du même enfermé dans la coque ou capsule; il semble entouré d'uno zone transparente. Grossissement, 170 diamètres.

\section{PLANCIE 7.}

Fig. 1. Lima squamosa, vu de face, pour montrer que l'ovaire $(0,0,0)$, d'un beau rouge, a envahi tous les organes qu'il masque et fait disparaître. Les organes de Bojanus $(r)$, dans lesquels s'ouvrent los ovaires, paraissent remplis d'œufs qui s'échappent par leurs orifices $(c)$.

Fig. 2. OEufs de la même. Grossissement, 170 diamètres.

Fig. 3. Portion des acini ou culs-do-sac sécréteurs de l'ovaire du Cardium rusticum. Grossissement, 400 diamètres. La capsule de l'œuf et son point d'attache sont remarquablement évidents.

Fig. 4. OEufs du Spondylus Gederopus pris à leur sortie de l'ovaire à la suile d'une ponte que j'avais déterminée. Grossissement, 170 diamètres. 
Fig. 5. Parenchyme cellulaire du cul-de-sac sécréteur de l'ovaire de l'Unio pictorum. Grossissement, 170 diamètres.

Fig. 6. Id. des œufs se développant dans les cellules. Grossissement, 170 diamètres.

Fig. 7. OEufs sans capsule apparente. Grossissement, 170 diamètres.

Fig. 8-9. OEufs avec une capsule qui n'est évidente que vers le point d'insertion. La membrane vitelline fait dans cet endroit un peu hernie. Grossissement, 170 diamètres.

Fig. 10. La capsule devient évidente à l'opposé du hile d'insertion. Grossissement, 170 diamètres.

Fig. 11. OEuf mûr rompu à dessein pour montrer que, entre la capsule et l'œuf, il y a bien un liquide, puisque les granulations vitellines s'y sont répandues. Grossissement, 170 diamètres.

Fig. 12. Epithélium vibratile des conduits de l'ovaire de l'Unio pictorum. Grossissement, 400 diamètres.

Fig. 13. Coupe théorique des acini de l'ovaire du même, pour montrer l'origine des œufs dans les parois et leur saillie dans la cavité.

Fig. 14. Spermatozoïdes de l'Unio littoralis, leur cellule productrice (e), les cellules mères des cellules productrices $(a, b, c, d)$. Grossissement, 500 diamètres.

Fig. 15. Spermatozoïdes de la Trigonella piperata. Grossissement, 500 diamètres.

\section{PlaNCHE 8 .}

Fig. 1. Exemple de Pecten varius dans lequel la glande était presque réduito aux canaux excréteurs ; $\left(a^{\prime}\right)$ rameau de lobe sus-hépatique ; $(a)$ rameau du lobe abdeminal ; (b) orifice dans le sac de Bojanus ; $(c)$ orifice de ce dernier. Double de grandeur naturelle.

Fig. 2. Spermatozoïdes du Pecten varius. Grossissement, 500 diamètres.

Fig. 3. Id. du P. glaber. Grossissement, 5000 diamètres.

Fig. 4. Pecten glaber. $(a)$ conduits excréteurs du testicule et de l'ovaire; $(d)$ petits îlots de glande femelle isolés au milieu de la glande mâle; (b) orifice commun aux organes des deux sexes, et placé dans l'organe de Bojanus qui s'ouvre en $(c) ;(o)$ ovaire; $(t)$ testicule.

Fig. 5. Huitre comestible. (b) orifice génital qui termine le conduit excréteur; (g) ganglion branchial; $(x)$ connectif branchio-buccal; $(n)$ nerf branchial. Entre les deux cordons nerveux se trouve l'orifice de la génération. - Ce dessin a été avec intention forcé pour rendre plus net cet orifice, méconnu par tous les auteurs. Si, dans la nature, il ne paraissait pas aussi distinctement, je renvoie aux indications du texte pour le découvrir.

Fig. 6. Un ouf enveloppé dans sa capsule. Grossissement, 170 diamètres.

Fig. 7. Acini, culs-de-sacs sécréteurs d'un individu presque entièrement mâle.

Fig. 8. Paquets spermatiques au milieu des granulations qui s'échappent de 
la glande. (a) les paquets de cellules qui correspondent aux paquets des filaments.

Fig. 9. Spermatozoïdes de l'Huître, au moment où ils deviennent libres. Grossissement, 500 diamètres.

\section{Planche 9.}

Fig. 1. Une grappe ou lobule du testicule d'un Cardium rusticum. Grossissement, $\mathbf{5} 5$ diamètres.

Fig. 2. Coupe de quelques acini du même, pour montrer les produits de la sécrétion tombés au centre des conduits en cul-de-sac, dont la paroi est tapissée par le parenchyme cellulaire.

Fig. 3. Spermatozoïdes du même. Grossissement, 500 diamètres.

Fig. 4. Aspect des dernières ramifications des testicules $(a)$ au-dessus du foie (b), dans la Venus decussata.

Fig. ॐ. Un lobule du testicule du même, pour montrer les culs-de-sac remplis de filaments et les parois tapissées par le parenchyme cellulaire.

Fig. 6. Partie du même. Grossissement, 240 fois. On distingue plus nettement que dans la figure précédente la couche cellulaire externe et les paquets spermatiques en dedans.

Fig. 7. Un paquet spermatique du même. Grossissement, 500 diamètres.

Fig. 8. Les filaments isolés du même. Grossissement, 500 diamètres.

Fig. 9. Cellules productrices des filaments du même. Grossissement, 500 diamètres.

Fig. 10. Filaments spermatiques et cellules du testicule de la Gastrochcona dubia Tarentina. Grossissement, $\mathbf{5} 00$ diamètres.

Fig. 11. Filaments et cellules de la Corbula striata. On voit des cellules mères.

Fig. 12. Partie d'un cul-de-sac sécréteur de la Cardita sulcata. Les spermatozoïdes en $(d)$ semblent encore enfermés dans la cellule qui les a produits et en être en partie ou complétement dégagés en $(c)$. Grossissement, 500 diamètres.

Fig. 13. Extrémité de l'abdomen du Spondylus Gaderopus, grossi deux fois pour montrer l'aspect de la glande mâle.

Fig. 14. (a) Masse spermatique dont se dégagent les filaments; (b) filaments isolés de la Lima squamosa.

Fig. 15. Cellules mères, cellules productrices des filaments, et filaments spermatiques de la Petricola Ruperella. Grossissement, 500 diamètres.

PARIs. - Imprimerie de L. MARTINET, rue Mignon, 2. 




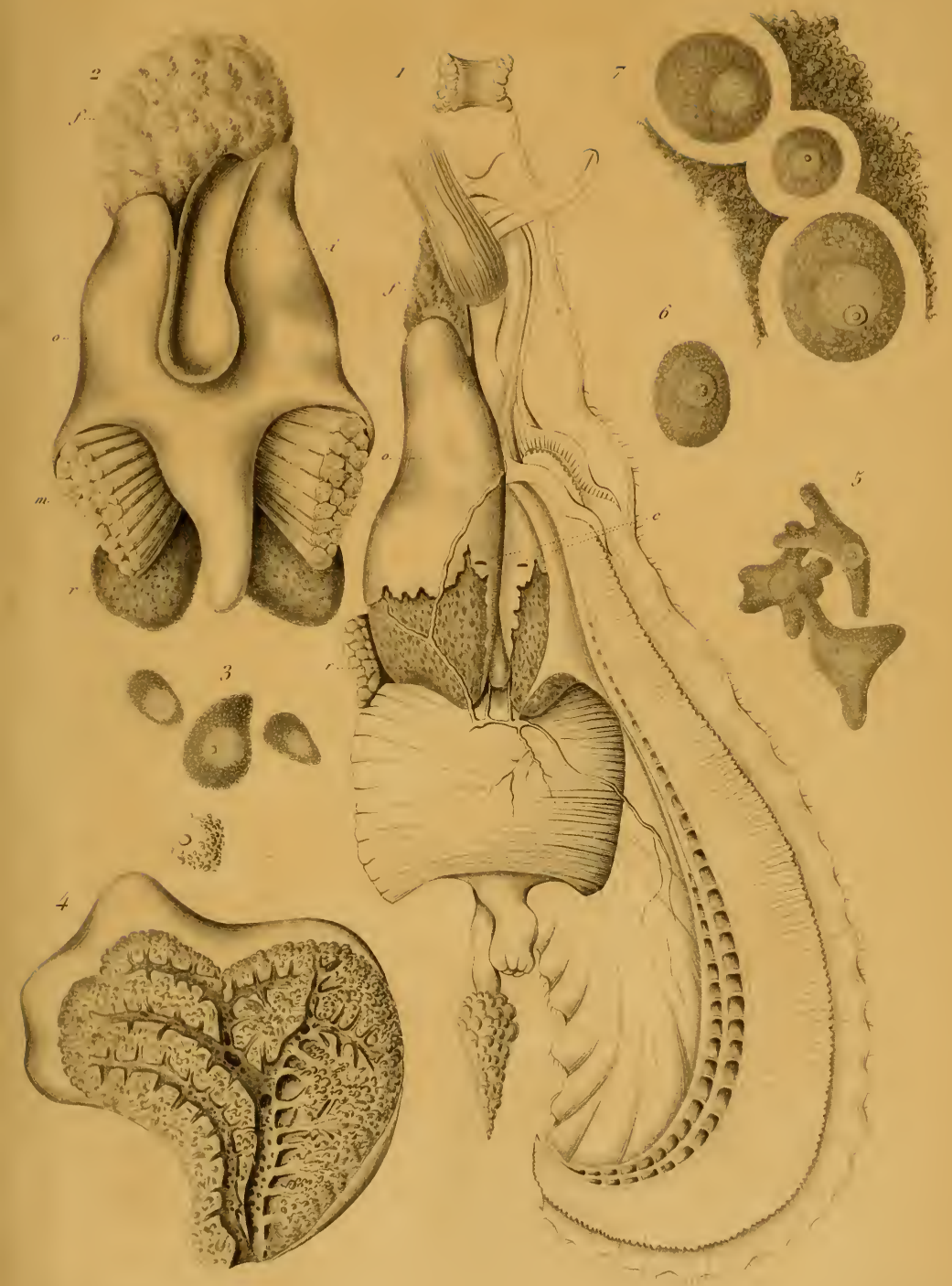




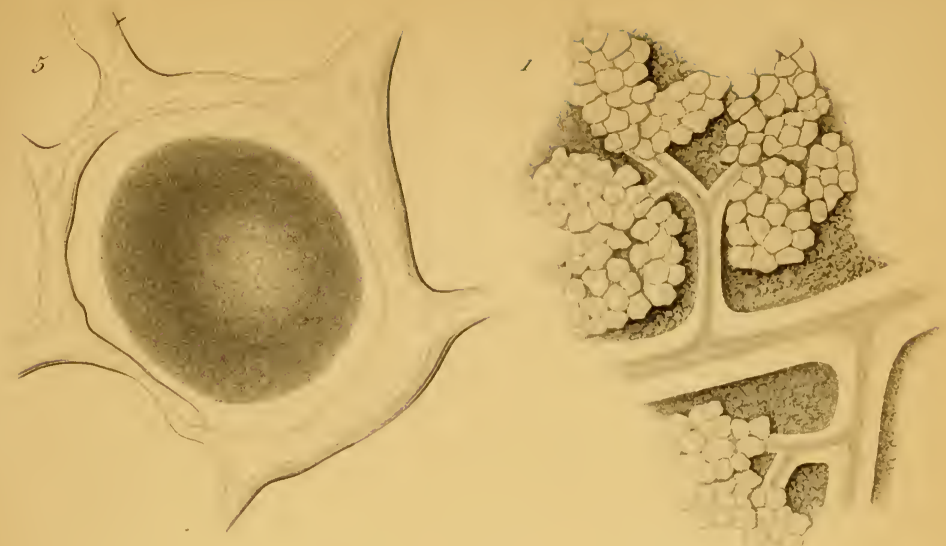

2

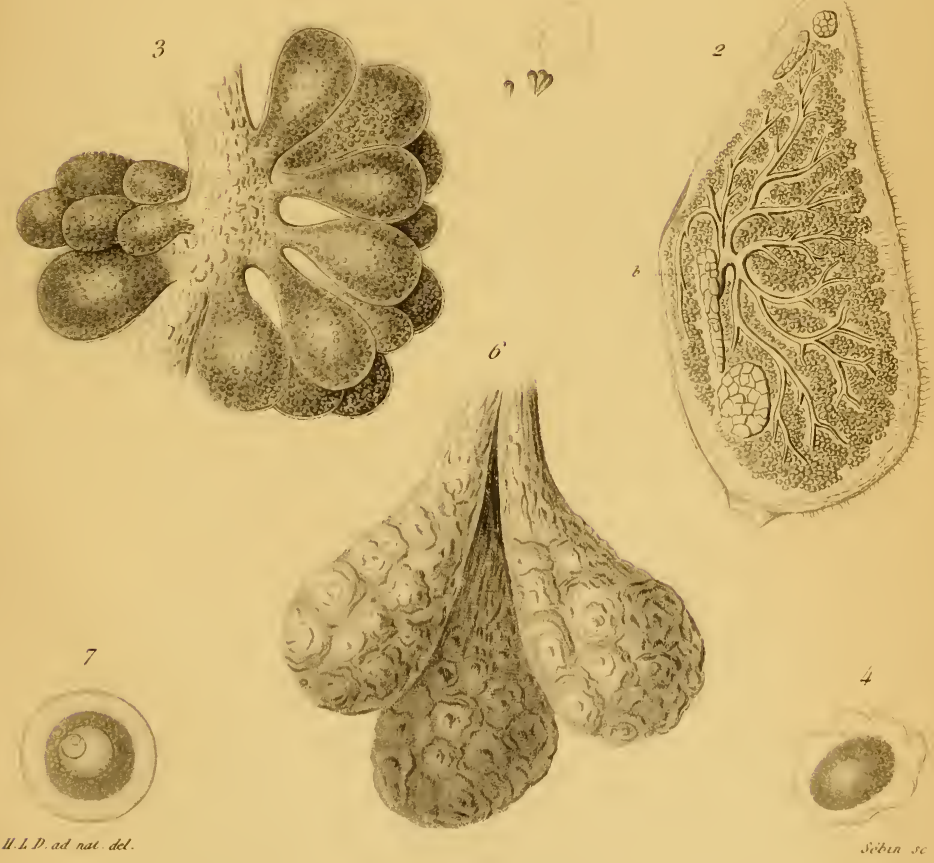

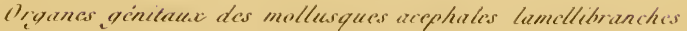




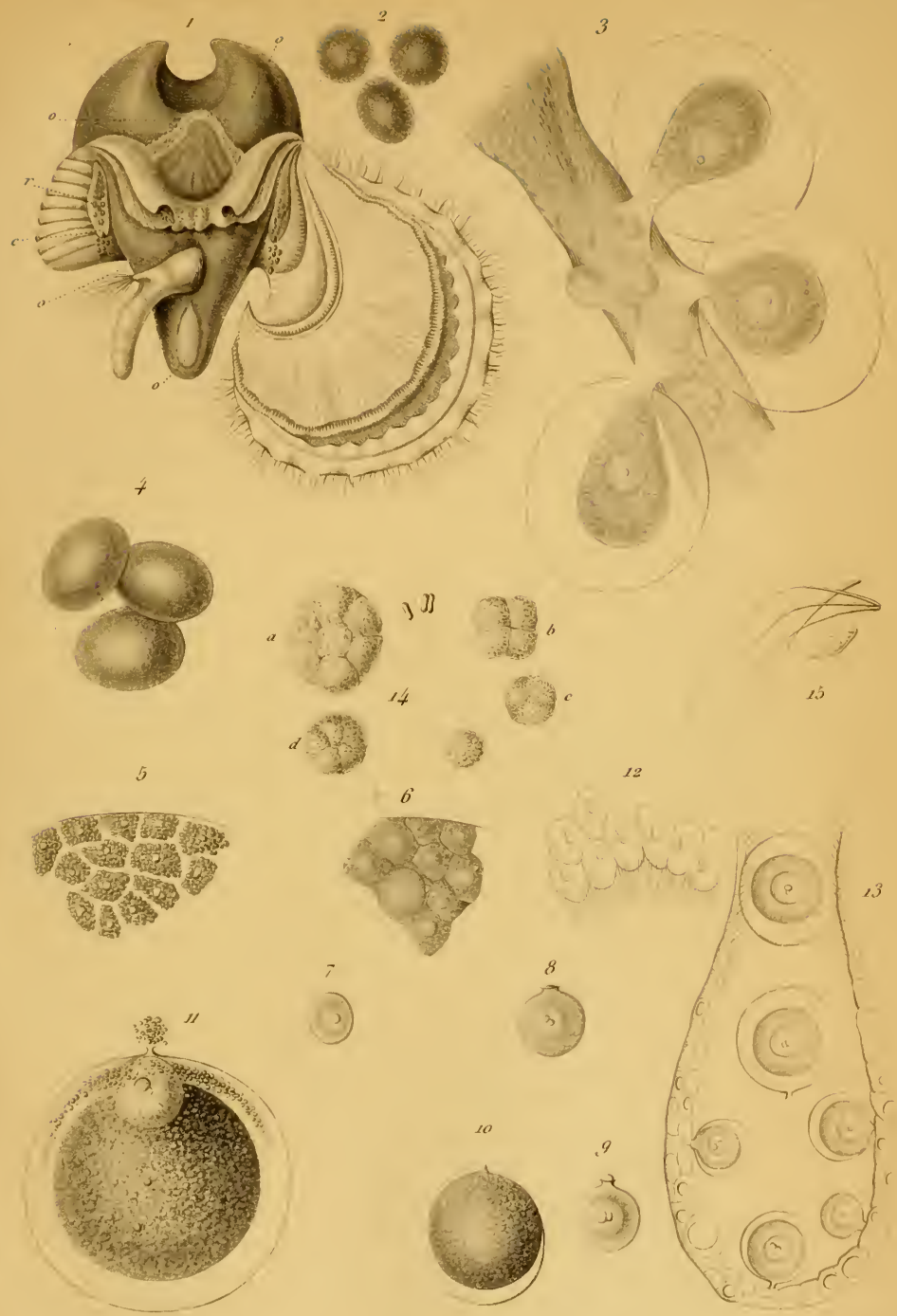

H.L D ad nat del

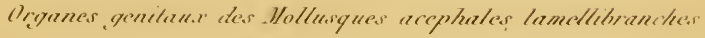





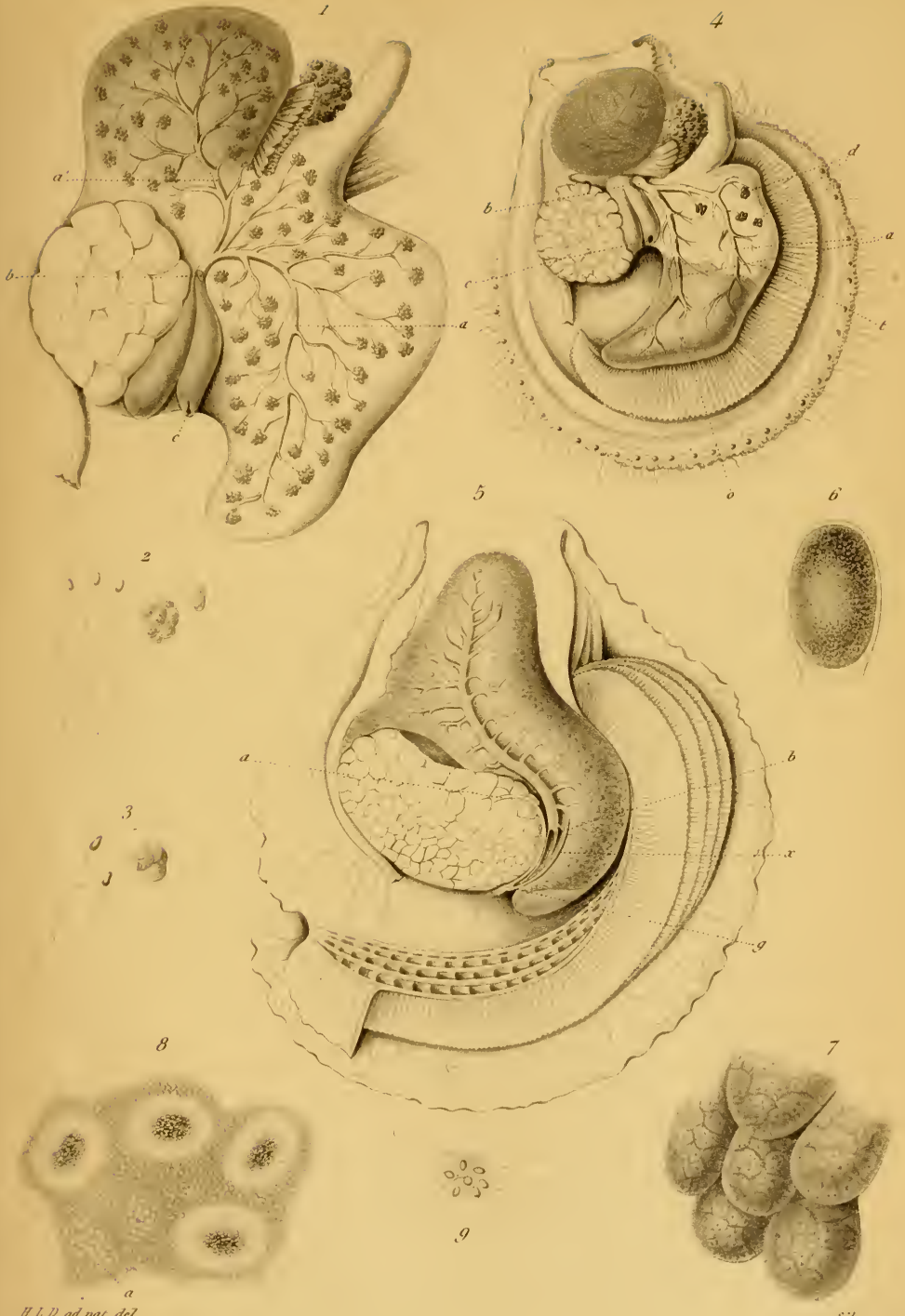

II L D ad mas dcZ 



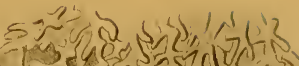

4 - J $\rightarrow$ an wa

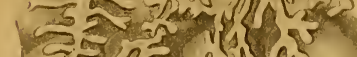
(.) 105 री दि

a 305 of an 9$\}$ P

5
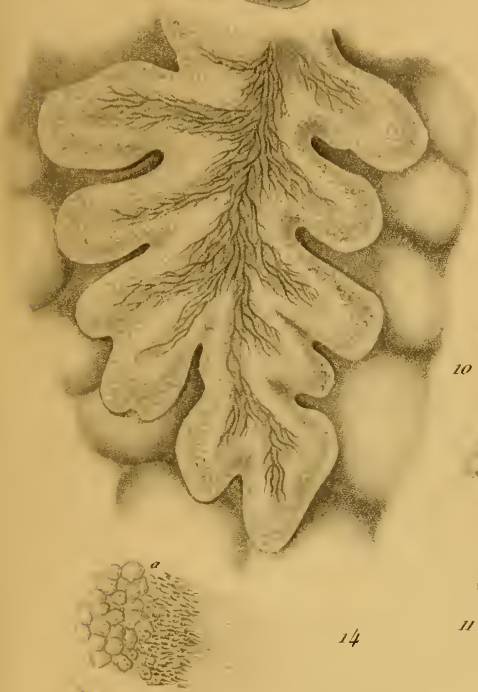

14

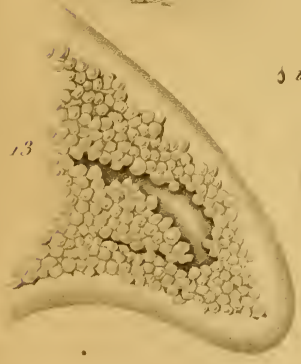

jo 35

$\pi$
$?$

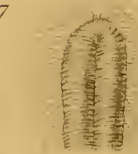
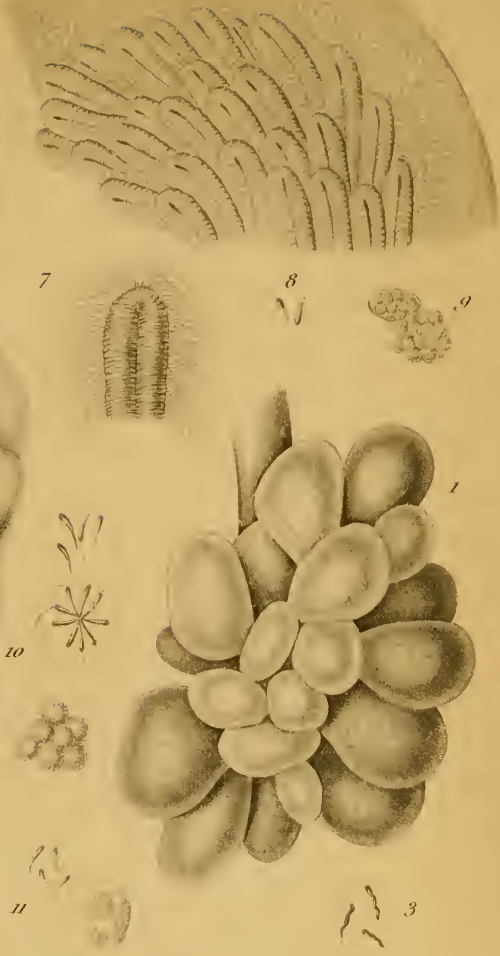

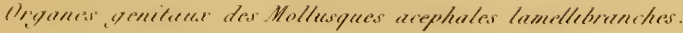





\section{MEMOIRE}

sur

L'ORGANE DE BOJANUS DES AGÉPIALES LAMELLIBRANGHES.

I.

Dans presque tous les Acéphales lamellibranches, on trouve de chaque côté du corps, vers l'insertion des branchies, entre l'abdomen, le muscle postérieur des valves, le cœur et le foie, une glande plus ou moins brunâtre, sur les fonctions de laquelle on est loin de s'entendre.

Bojanus (1) s'en est occupé le premier avec suite; cela explique pourquoi on la trouve souvent désignée par ces mots corps, sac, organe de Bojanus. Il la regardait comme l'organe de la respiration; Poli (2) pensait qu'elle sécrétait la coquille ; Neuwyler (3) la considérait comme le testicule; Treviranus (4), Carus (5), Garner (6), R. Owen (7), von Siebold (8), admettent qu'elle est le rein; MM. Deshayes (9) l'appelle l'organe dépurateur; Van-der-Hœven (10) et von Siebold (11) aussi, lui

(1) Bojanus, Isis, 1819 , p. 46 , pl. 1, fig. 1; 1820 , p. 404 .

(2) Poli, Testacea utriusque Sicilice historia et anatome, 1791-1795, int. p. 18 , t. II, p. 86 , pl. 20 , etc., p. 143 , pl. 26 , etc., p. 41, pl. 237.

(3) Neuwyler, Die Generations-organe von Unio und Anodonta, dans les Neue Deushrift. der Allgem. Schw. Ges. für die gessammt. Natur. , VI, 1812, p. 1, pl. $1-3$.

(4) Treviranus, dans Tiedemann, Zeitsch. fiir Physiol., I, p. 53.

(5) Carus, Zool., 1834 , II, p. 650.

(6) Garner, Trans. of the phil. Soc., p. 92, t. II, pour 4841.

(7) R. Owen, Lect. on the comp. Anat., p. 284.

(8) Von Siebold, Anat. comp., t. I, $2^{\mathrm{e}}$ partie, p. 280, not. 5.

(9) Deshayes, Exploration scientifique de l'Algerie, les diverses Monographies.

(10) Van-der-Hœven, Meckel's Archiv., 1828, p. 502.

(11) V:Siebold, loc. cit., P. 281 , note 6. 
trouvent beaucoup d'analogie avec les appendices veineux des Céphalopodes.

Il est peu d'organes dont le rôle ait été, on le voit, plus diversement interprété.

En face de tant d'opinions, il eût été difficile de prendre une lécision absolue, surtout quand les faits n'avaient pas un caractère tel qu'ils pussent trancher nettement la question; aussi le travail que je puhlie aujourd'hui est-il plus anatomique que physiologique. Pour avoir une opinion définitivement arrêtée, de nouvelles recherches, où la chimie physiologique eût joué le plus grrand rôle, étaient nécessaires. Des circonstances ne m’ont pas permis de les entreprendre ; j'ai dû cependant faire connaître les particularités qui, pendant un examen fréquemment répété, se sont présentées à mon observation. La structure de cette glande ćlait d'ailleurs peu connue, et il existait quelques erreurs anatomiques à son égard, et une ćtude comparative sur un nombre suffisant d'espèces manquait absolument. Il y avait done lì quelque ('hose à faire; aussi le but de ma publication est-il principalement de combler une lacune. Si je présente à la fin quelques considérations sur la physiologie, elles doivent être regardées seulement comme des éléments pouvant servir plus tard à une étude approfondie des fonctions.

Je place la description de l'organe de Bojanus à côté et après celle des organes de la reproduction, parce qu'il m'a paru y avoir un rapport constant entre les deux. Toujours ceux-ci s'ouvrent ou dans l'intérieur de celui-lì, ou à côté; et ce rapport des orifices excréteur's conduit à̀ un rapprochement analogue à celui que l'on fait dans les animaux supérieurs, entre les glandes rénales et celles de la reproduction.

On trouvera done en définitive dans ce travail une étude détailléc de la structure de l'organe, son anatomie descriptive, des données curieuses sur l'origine de quelques concrétions en forme de perles, et une appréciation des principales opinions des auteurs. 
II.

AXATOMIE.

$\S 1^{\text {er }}$.

Description de l'organe.

Le sac de Bojanus est toujours facile à trouver; sa position est à peu près constante, mais sa forme, ses rapports et ses autres caractères, varient avec les espèces.

La teinte suffit le plus souvent pour le faire reconnaitre. Quand on écarte les branchies après avoir placé l'animal sur le dos (1), e'est-à-dire sur la partie qui correspond à la charnière de la coquille, on voit de chaque côté de la masse viscérale, habituellement blanchâtre, ou grisâtre, ou plus ou moins rouge, un corps oblong, qui se fait distinguer par sa coloration presque constamment brunâtre. Cette teinte cependant est dans quelques cas différente : ainsi dans la Lime squameuse, elle est d'un jaune clair; dans l'Anomie de la Méditerranée, je l'ai vu souvent d'un violet assez riche en coloris. Du reste, son intensité varie avec les individus, et probablement aussi avec les époques de l'année.

On verra plus loin l'explication de cette différence; toujours estil qu'à part quelques exceptions, c'est entre le brun foncé, le brun olivâtre ou le jaune verdâtre, qu'eile varie.

La forme dépend de celle du corps de l'animal ; elle change avec celle-ci. On ne peut, du reste, bien en juger qu'en enlevant soigneusement les branchies et tous les tissus blanes qui les unissent au péricarde, au cour et au manteau. Alors on voit que le sac occupe tout l'espace compris entre la poche péricardique en dessus, le muscle postérieur des valves en arrière, le foie en avant, la masse glandulaire génitale en avant encore, mais en bas et en dedans, les muscles rétracteurs postérieurs du pied en dessous. L'espace dans lequel est comme enchâssée la glande détermine

(1) PI. 5, t. IV, 4 e série, fig. 6 (Cardium rusticum), vu de face en dessous $(r)$. 
naturellement sa forme; que l'animal soit allongé, et elle sera longue comme dans les Modioles (1), les Moules, l'Arche(2); qu'il soit, au contraire, court et ramassé sur lui-même, et la forme deviendra irrégulièrement globuleuse, comme c'est le cas le plus fréquent.

Les proportions du musele des valves, celles de la masse viscérale abdominale et des muscles du pied, causent aussi de très grandes variations dans la disposilion; ainsi, dans les coquilles des Pèlerins, où le muscle des valves est si volumineux et où la masse abdominale s'étend beaucoup en arrière, les deux saes font saillie dans les dépressions qui separent les deux parties. Dans les Lucines, l'extrémité postérieure se contourne sur le muscle des valves; aussi semble-t-il que le corps de Bojanus présente trois branches. Dans la Nacre (3), la masse de l'organe est globuleuse, et bien neltement limitée de chaque côté.

Ces variations de forme n'ont rien d'important; elles correspondent toutes aux variations que présentent les animaux. Ce dont on juge très bien dans l'Anomic par exemple, où l'un des côtés du corps est fortement contourné, tandis que l'autre présente la forme, habiluelle. L'une des glandes décril une courbe d'un très court rayon, tandis que l'autre est presque droite.

Les rapports sont importants à connaitre; ils présentent des différenees qui tiennent encore à la disposition générale.

Dans presque tous les cas, le sac de Bojanus s'applique en arrière sur le muscle des valves : c'est lì un rapport constant (4), Quand il remonte sur le dos, il contracte un nouveau rapport; il s'approche du rectum; quelquefois celui-ci, après s'être dégagé du cœur, se loge en glissant sur la face dorsale du muscle des valves entre les denx saes; enfin le péricarde tapisse la partic dor-

(1) Voy. Ann. des sc. nat., $4^{\circ}$ série, 1854 , t. IV, pl. 5, fig. $10(r)$.

(2) Je renvoie, quand la spécification n'est pas donnée en note, à la liste des espèces qui a été publiée en 1854, Annales des sciences naturelles, $4^{\mathfrak{e}}$ série, $\mathrm{t}$. II, p. 242 et suiv.

(3) Voy. Ann. des sc. nat., $4^{\mathrm{e}}$ série, 1854 , t. II, pl. 5, fig. 2.

(4) Voy. id. t. II, pl. 5, fig. 1; pl. 7, fig. 1; pl. 8, fig. 14; t. IV, pl. 5, fig. (2), (6), (10), 113). 
sile des deux glandes, qui forment comme le plancher inféricur de la cavité où se loge le cour (1).

Les cordons nerveux, qui unissent les ganglions buceaux aux ganglions branchiaux, sont toujours en rapport inmédiat aver l'organe; ils sont placés contre sa face interne, et dans les exemples où le corps est allongé, comme dans les Mulettes, les Anodontes, on doit les ehercher au milieu de la substance même de la glande; la couleur blanehe de ceux-ci tranche vivement sur la teinte si brune du sac de Bojanus.

Les branchies sont le plus souvent insérées sur une ligne qui partage en deux parties à peu près égales la glande. Ainsi dans le Peigne varié, le Peigne glabre, et bien d'autres qu'il serait trol' long de citer (2), lorsqu'on les a enlevées, on voit leur insertion qui se marque par un sillon. Cependaut dans la eoquille de SaintJaeques et dans la Lime, la branchie est insérée en dehors; aussi la glande se trouve-t-elle dans ees espèees entre la masse abdominale et l'organe respiratoire (3).

Les rapports qu'affeetent les deux sacs l'un avec l'autre sont importants ; ils varient eomme préeédemment et dans les mêmes cireonstances. Ainsi dans les cas où la masse viscérale s'avanec beaucoup en arrière, elle empêche les deux extrémités inférieures des deux glandes de se réunir sur la ligne médiane; on voit alors eelles-ci, séparées et distinctes, former deux corps, l'un ì droite, l'autre à gauche; il n'est question ici que de la partie apparente, sans autre préparation que l'éeartement des branchies. Cette disposition existe à son maximum dans la lime squameuse (4).

Dans le cas coutraire, les deux saes peuvent se rapproeher en bas sur la ligne médiane, et former eomme un collier autour dı

(1) T. IV, pl. 6, fig. 3, 4 .

(2) Je renvoie, du reste, aux figures qui ont été publiées dans les Annales, sur les organes de la reproduction. Voyez cependant particulièrement la figure 1 de la planche $8, t$. II.

(3) Ceci est très marqué dans la figure 2 de la planche 6 , t. IV. La masse abdominale est d'un côté, les branchies de l'autre.

(4) T. II, pl. 7, fig. 1. 
muscle du pied, sorte de pédicule de la masse abdominale; cela s'observe dans le Cardium rusticum, la Lucina lactra, ete.

Plus profondément, les deux glandes se rapprochent constamment du côté du dos. Dans l'Anodonte, la Mulette des peintres, la Lutraire solénoïde, la Bucarde frangée, la Pholade, etc., la disposition est si nettement marquée, qu'en observant l'animal du côté du péricarde, après avoir enlevé le cœur, on croirait à l'existence d'une seule masse glandulaire (1).

Il y a même accolement des parois internes sur la ligne médiane et formation d'une cloison verticale, qui le plus souvent est percée d'une ouverture (2), ètablissant une communication directe entre le sac de droite et le sac de gauche. Je reviendrai sur cette disposition en décrivant la cavité de la glande.

A l'intérieur, la glande est assez compliquée, et sa disposition n'a été que bien imparfaitement connue et décrite.

Prenons l'exemple même étudić par Bojanus, l'Anodonte, l'Anodon cygnaum, comme il l'appelle. Du reste, dans la Mulette des peintres, dans la Lutraire solénoïde, la Pholade, les Bucardes, les Corbules, etc., les choses sont semblables.

$\mathrm{Si}$, après avoir enlevé les branchies, on incise l'organe suivant sa longueur, un peu de côté, on tombe dans une large poche où la teinte est encore plus marquée qu'au dehors ; on y voit appliqué sur la paroi interne une élévation qui fait saillie dans la cavité; c’est cette masse saillante que Bojanus avait considérée comme la partie active de l'organe, comme le poumon. Une connaissance plus exacte et plus complète de la disposition eût empêché d'avancer une telle opinion. En effet, en déchirant cette partie saillante, on trouve dans son intérieur une nouvelle cavité. Bojanus avait vu cette cavité, mais il la croyait close; ce qui n'est pas, car elle présente deux orifices très distincts. Sur un animal ouvert avec précaution, qu'on fasse une petite ouverture à la paroi de cette cavité interne, et puis qu'on pousse une matière colorante dans son intérieur, on verra cette matière s'échapper dans la première poche la plus extérieure, et

(1) T. IV. pl. 6, fig. 3, Cardium.

(2) T. IV, pl. 6, fig. 4, Lutrario 
cela en arrière. Si l'on cherche aver soin dans la partic où s'est échappé le liquide, on trouve au milieu des replis glandulaires qui lapissent les parois, et le cul-de-sac voisin dı muscle postérieur des valves, une très large et longue fente yui établit la communication entre les deux cavités. On ne peut guère donner ì cette large communication le nom d'orifice; car elle a presque antant d'étendue que le diamètre du sac lui-même; elle est masquée par les replis glandulaires dont les interstices lui ressemblent, се qиi certainement a empêché de la distinguer.

S’il était encore besoin de critiquer l'opinion de Bojamus, on trouverait ici un argument bien fort contre sa théoric. Comment admettre, en effet, que la cavité centrale dı poumon pourrait ètre remplie de sang, alors qu'elle communique avec la poche périphérique où, d'après l'auteur, entre l'eau servant à la respiration.

En étudiant la partie de la glande qui forme le plancher du périearde, on est frappé de l'existence de deux dépressions qui se trouvent en avant (1), tout près de l'endroit où le rectum sort de la masse viscérale pour pénétrer daus le cœur. En présentant li tête d'une épingle à insecte dans ces dépressions qui ressemblent à des orifices de quelques conduits, on la voit bientôt disparaître, et pénétrer assez avant. On cherche naturellement si l'épingle n'a pas pénétré daus l'organe de Bojanus placé au-dessous, et l'ou I'est pas peu surpris de la rencontrer dans la cavité interne de la partie saillante, appelée poumon par Bojanus.

Ce fait, par lui-mème assez singulier, méritait d'être vérifié sur d'autres espèces; il élait curieux de savoir si c'était une disposition exceptionnellement propre à l’Unio et à l'Anodonte; aussi, dès que je pus me rendre sur les bords de la mer, ce fut une (juestion que je me proposai de résoudre. L'année dernière (1854) et cette année (1855), j’ai pu observer sur les còtes de Bretagne deux espèces de Bucardes, la Dentelée el la Tuberculeuse, une Pholade d'une taille fort considérable, la Lutraire solénö̈le, la Corbule striée, et j’ai eu la satisfaction de voir se généraliser le fait que j’avais observé à Paris. Plus tard, sur les Anodontes des envirous de Lille, j’ai tronvé, il fillail s'y attendre, une semblable disposi-

(1) T. IV, pl. 6, fig. 3. Péricarde ouvert, le cœur enlevé. Cardium. 
tion. Garner avait vu cette eommunication dans l'Unio ; il la cite, mais il ne l'a pas cherchée dans d'autres espèces. Du reste, le peı de détail qu'il donne laisserait croire (qu'il n'a point eu une connaissance complète de toutes les dispositions.

Si maintenant nous cherchons à considérer dans leur ensemble ainsi que dans leurs rapports les différentes cavités et leurs orifices, nous verrons que l'entrée du sac est placée à côté de l'orifice de la génération (1), en avant, dans l'angle où s'insère l'extrémité antérieure des branchies; qu'à cet orifice d'entrée fait suite une grande eavité (2), quej'appellerai périphérique. dans l'intérieur de laquelle s'ouvre en arrière la cavité centrale (3), qui elle-même communique en avant, et tout près de l'orifice externe ( 4$)$, avec la poche péricardique (5).

La partie qui met en communication le péricarde avec l'organe de Bojanus est étroite, en forme de canal, et ne présente plus l'apparence glandulaire; elle passe en dessous de la fenêtre de communication des deux saes, en croisant sur son côté interne l'extrémité antérieure du sac périphérique, tout près de son orifice excréteur ou externe.

Ainsi le péricarde communique avec l'extérieur par l'intermédiaire du sac de Bojanus, et le trajet que parcourt le sac, pris dans son ensemble, est une courbe aplatie, rentrante, dont le point d'arrivée (orifice externe) (6) est voisin du point de départ (orifice péricardique) (7); l'un est supérieur, l'autre est inférieur.

Cette communication du péricarde n'a pas été connue des anatomistes, Garner seul en parle pour l'Unio dans un passage très court de son Mémoire sur les A céphales lamellibranches.

Il faut toutefois se garder de généraliser trop vite.

(1) T. IV, pl. 5, fig. 2 (pe. ov.). Coupe un peu théorique du corps de Bojanus dans la Mulette des peintres.

(2) Id., fig. $2(c p)$.

(3) Id., fig. $2(c c)$.

(4) Id, fig. $2(p i)$.

(b) Id., fig. "2 (r).

(6) Il. ";, fig. $2(\nu c)$.

(7) Id., fig. $2(p i)$. 
Je dois dire, en effet, qu'on éprouve de grandes difficultés ì recomnaitre si le péricarde communique avec le sac de Bojanıs, lorsque certaines dispositions se présentent comme dans les Peeten, où on ne trouve plus les deux cavités que j’ai cherché à décrire plus haut, et où il n'y a évidemment qu'une seule poche. On sait que, dans cet animal, les oreillettes s'avaneent vers les branchies, assez loin du cour proprement dit. Le péricarde s'allonge, et forme un cul-de-sac très étroit, qui se place à côté du sac de Bojanus. Il est fort difficile de pouvoir s'assurer si, au fond de cet étroit eonduit, il existe une communication; malgré tous mes efforts, je n'ai pu arriver à une conviction qui ne laissâl point de doute.

Quant aux rapports des deux sacs, ils sont plus intimes ici que dans les exemples précédents. Dans le Pecten jacobaus, les deux organes semblent se eontinuer (1) l'un avec l'autre par une portion membraneuse sous-péricardique, qui n'a plus l'apparence glandulaire; aussi les deux organes réunis forment-ils un tout placé comme à elieval sur la dépression qui se trouve entre lo muscle des valves et la masse viscérale; les deux portions véritablement glandulaires pendent de chaque côté de la masse abdominale, entre elle et les branchies.

Je n'ai pas vu non plus dans l'Huitre vermeille cette partie centrale, dont la cavité s'ouvre dans le péricarde. Je dois dire que, lorsque je pouvais disséquer cette espèce, je n’avais pas encorc trouvé la communication dont je parle; toutefois, d'après les dessins que j'ai faits et en particulier d'après celui que je publie (2), on reconnaitra la plus grande analogie avec ce qui s'observe dans le Pecten (3), et si la communication avec le péricarde peut m'avoir échappé, il me parait difficile que la partie, considérée comme le poumon par Bojanus, ait pu passer inaperçue.

Il me semble qu'il y a, à l'égard de la disposition dont il s'agit ici, deux types bien différents : dans l'un, une cavité centrale. communique à la fois aver le péricarde et avee une cavité péri-

(1) T. IV, pl. 6, fig. I (z).

(2) T. IV, pl. 4, fig. 6 .

(3) T. IV, pl. 6, fig 3. 
phérique; celle-ei s'ouvre au deliors, et a une large communication avec celle du côté opposé; dans l'autre, le sac est simple, et s'ouvre d'une part au dehors, de l'autre dans le sac du côtó opposé.

Il est probable que cette grande différence est la conséquence d'une importante modification, qui a tellement déplacé les parties, que l'orifice profond, s'il existe, est deventi tris difficile à reconnaitre.

Enfin, dans la Moule comestible et la Nacre, on trouve des dispositions sur lesquelles je dois appeler l'attention.

Pour la Moule, von Siebold (1) s'exprime ainsi : " Les organes "urinaires sont encore plus singulièrement disposés..... Leurs "deux sacs, qui sont situés à la base des branchies, sont fendus » dans toute leur longueur, de sorte qu'en écartant les branchies, " on apercoit distinctement les compartiments et les cellules de ces "glandes. „Il renvoie à un travail de Treviranus (2), et semble formuler son opinion d'après celle de ce dernier. Il y a là une crreur que je me contenterai simplement de relever aujourd'hui ; peutètre un jour présenterai-je l'anatomie complète de la Moule, ear elle me paraît offrir, à bien des égards, de l'intérêt. Comme cet animal n'est pas rare, comme on le trouve toujours ef partout, il n'a éveillé la curiosité d'aueun malacologiste l'unc manière sérieuse.

Mais une particularité a causé l'erreur des auteurs allemands. Les vaisseaux sanguins, qui rapportent le sang du manteau aux branchies, passent sur un plan inférieur (3) au sac de Bojanus; entre chaque vaisseau, qui s'est comme détaché de la paroi du sac, sont des dépressions qui ont été prises pour les replis internes de la substance glandulaire; ce qui a conduit à admettre que le sac est ouvert d'un bout à l'autre. Si l'olsservation de la circulation ne suffisait pas pour faire reconnaître l'erreur, la présence même

(1) Manuel d'anatomie comparée, t. I, $2^{\mathrm{e}}$ partie, p. 279, note 2.

(2) Voy. Treviranus, Beobacht. aus der Zoot. und Physiol., p. 5I, s. 686.

(3) On n'oublie pas que lorsqu'on examine l'animal en le plaçant sur le dos, ce qui semble supérieur dans celte position est en réalité inférieur quand l'animal est en place. 
d'une cavité daus la glande, l'existence d'un orifice séeréteur, viendraient donner sans aucun donte raison à la manière de voir que j'expose ici.

Quant à la communication avec le péricarde, il m'a été impossible de m'assurer de son existence. La portion d'une partie centrale, analogue à celle de l'Anodonte, ete., ne m'a pas paru très évidente; je me réserve, du resteà cet égard, pour le moment où je présenterai l'anatomie de la Moule; je désirais ici seulement relever l'erreur qui existait.

Dans la Nacre (1), le corps glandulaire est très développé; je l'ai représenté dans le dessin que j'ai donné des organes génitaux de cet animal (2). Il forme deux masses noiruitres placées en avant du muscle postérieur des valves, et en arrière des organes génitaux. La teinte blanche du premier, celle rouge-brique des seconds, fait ressortir la couleur noire de celui-ci. Quand on eximine son intérieur, on trouve une disposition qui ne laisse pas que d'embarrasser un peu tout d'abord. En arrière de l'organe de la génération, sur la ligne médiane, en regardant l'animal en des sous et en fice, on voit une dépression où paraìt un petit lobule. antérieur : c'est l'entrée d'une arrière-cavité, d'un cul-de-sac qui s'avance vers le dos jusqu'au péricarde. Si l'on ineise, avec précaution sur la ligne médiane, la masse viscérale, de manière i ouvrir largement cette cavité, on remarque qu'elle est contiguë à trois poches : une dorsale, le péricarde, et deux latérales fort grandes qui remontent sur ses côtes et sur le dos des muscles postérieurs du byssus (3). Ces dernières contournent complétement les muscles, et reviennent en dehors s'unir au parties noirâtres qui sont véritablement le corps de Bojanus. Elles sont entièrement membraneuses, et semblent être le canal excré-

(1) Pinna nobilis.

(2) Voy. Ann. des sc. nat., $4^{\mathrm{e}}$ série, 1854 , t. II, pl. 5, fig. 1 et 2.

(3) Voy. pl. 5, fig. 1, 2, ou t. II, 1854, $4^{\mathrm{e}}$ série des Ann. des sc. nat. Toutes les membranes formant les cavités ont été enlevées dans les, dessins publiés. - On n'y voit plus que le lobule qui pend entre les deux glandes, ss' et surtout les deux muscles du byssus $(m)$; la cavité dont il s'agit ici remonterait donc sur la face dorsale de res muscles. 
teur de la glande démesurément dilaté. Elles représentent peutêtre à certains égards les poches périphériques que l'on a vues dans l'Anodonte.

Du reste, dans la masse noirâtre de l'organe, on trouve des plis épais de nature glandulaire, qui entourent une cavité profonde, moins étendue que la précédente, dans l'intérieur de laquelle celle-ci s'ouvre.

La communication avec le péricarde n'a pu être observée , car les individus frais manquaient pour cette étude. Ceux conservés dans l'alcool, que je dois à l'obligeance de M. le professeur Valenciennes et de M. Rousseau, aide-naturaliste au Jardin des plantes, ne m'ont pas fourni de renseignements suffisamment nets, pour que je puisse assurer que la communication existe.

Il doit, sans aucun doute, y avoir beaucoup d'antres variétés de forme et de disposition; mais je crois cependant que toutes, plus ou moins, se rapprocherout des deux types principaux, que les Bucardes, les Lutraires, etc., d'une part, les Spondyles, les Peignes de l'autre, nous offrent manifestement.

Voyons où et comment s'ouvre l'organe dont nous connaissons la disposition générale. C'est maintenant le lieu de nous occuper des orifices de la génération. Je disais, en effet, dans le travail où je présentais l'histoire des organes de la reproduction, que leurs ouvertures à l'extérieur offraient des rapports si intimes avec eeux du corps de Bojanus, que je renvoyais au moment où je m'oceuperais de celui-ci pour faire une étude détaillée de ces orilices; il est done indispensable de consulter les planches qui se rapportent à la génération (1).

Trois dispositions principales se présentent : tantôt les glandes de la reproduction s'ouvrent dans le sac de Bojanus, tantôt elles ont un orifice unique et commun avec celui-ci, tantôt enfin deux orifices distincts, et plus ou moins éloignés, appartiennent en particulier à chacune des glandes.

Examinons successivement ces trois cas.

(1) Voyez les pl. 5, 6, 7, 8, 9, t. II, Ł $^{\mathrm{e}}$ série, Ann. des sc. nut., 1854; et pl. $4,5,6$, t. IV, même série. 
Dins les spondyles et les Pcignes, il est très difficile de découvrir les orifices génitaux. Par une sorte de fatalité, la première espèce sur laquelle je cherchai ces conduits fut le Spondyle, et j'employai plus de huit jours de recherches délieates el pénibles a ne pas trouver une chose que le hasard me fit rencontrer an moment où je m'y attendais le moins. J'avais ouvert le sac de Bojanus, à peu près comme dans la figure que je donne, pour en étudier la structure, quand je vis sortir, par un orifice (1) situé du côté interne, dans une partie moins glandulaire que le reste dı sac, un cylindre rose, absolument de la couleur de la glande dont j'avais fait déjì l'étude. L'examen microscopique me montra bien vite que j’avais affaire à des oufs retenus dans une sorte de gelée albumineuse. Plus tard, je renouvelai l'observation, et dés ce moment, quand les orifices de la génération ne paraissaient point au dehors, je les cherchais dans l'intérieur même du sac sur la face interne, et c'est ainsi que j'ai pu les trouver dans les Limes, les Peignes bigarrés, de Saint-Jacques, à côtes rondes et glabres, alors que von Siebold et M. Humbert n'avaient pu les reconnaître.

Ce cas n'est évidemment pas le plus fréquent, au moins à ne considérer que les espèces étudiées.

Dans les Nacres ou Jambonneaux, l'orifice génital est presque à côté (2) de celui du sac de Bojanus; et ici, comme précédemment, les produits de la génération el ceux de la glande qui nous occupe sortent par un seul et unique orifice. C'est ce qui a fait dire à von Sicbold qu'il y avait là comme un petit cloaque.

En ouvrant par-derrière la grande poche membraneuse et non glandulaire decet animal, on voit les deux orifices très voisins, et l'on comprend que les oufs puissent tomber dans ce grand réscrvoir.

Dans l'Arche (3), l'organe de la génération s'ouvre bien près de l'orifice externe, dans l'intérieur du canal excréteur de l'organe de Bojanus, par une fente en boutonnière. L'orifice unique cxterne est porté ì l'extrémité d'une papille fendue en long à son extrémité,

(1) Voy. t. IV, pl. 4, fig. $6(o v)$.

(2) Voy. t. II, pl. 5, fig. 1 (c c).

(3) Voy. t. IV, pl. 5, fig. 3. 
et placée ì peu près vers le milieu de la longueur, de l'organe. La même disposition s'est présentée dans la Vodiole ou Dattile des Mahonais (1).

En résumé, on peut dire que, dans ce premier cas, les deux glandes s'ouvrent par un seul orifice, et que le conduit de l'un, dans des proportions très variables, sert de canal exeréteur ì l'autre.

Où se trouve done l'orifice le plus extérieur, celui, en définitive, qui appartient au sac de la glande que nous étudions? J'ai indiqué dans l'histoire des organes de la génération un rapport important que présente cet orifice avec le connectif nerveux, qui, des masses ganglionnaires branchiales, se rend à celles placées dans le voisinage de la bouche. C'est constamment en dehors du connectif, vers le point où il plonge dans la masse ahdominale (2), que l'on aperçoit l'oritice. Daus les Peignes, les Spondyles, les Limes, l'orifice génital étant profondément placé, conserve néanmoins ses rapports; mais celui du sae de Bojanus se trouve placé à l'extrémité postérieure, non loin des ganglions branchiaux (3).

Quand l'orifiee de l'organe est placé au sommet d'une papille, celle-ci oceupe presque toujours le milieu de la largeur de la glande, ainsi que cela s'observe dans la Modiole, l'Arche, la Moule, etc.

Voyons maintenant le troisième cas, celui où les orifices peuvent être plus ou moins rapprochés, mais jamais confondus.

Je prendrai d'abord l'exemple que nous avons étudié en commengant. Dans l'Unio et l'Anodonte(4), les orifices distincts sont placés côte à côte l'un de l'autre, tout à fait à l'extrémité antérieure du sac; on voit que la position est complétement opposée à celleque nous avons trouvée dans le Peigne. Dans des individus de belle taille ees deux orifices, fendus en boutonnières presque parallèles à l'axe du corps, sont longs d'un millimètre; ils sont très évidents, aussi n'out-ils échappé à aucun observateur : celui de la génération est

(1) Voy. t. IV, pl. 5, fig. 10 (ov).

(2) Voyez pour ce rapport sur les planches $5 \mathrm{du} \mathrm{t}$. IV, et en particulier, les fig. 6, Cardium rusticum; fig. 11, Petricola rupella; fig. 12, Cardila sulcala.

(3) Voy. pl. 6, t. IV, fig. 1, 2 (pe).

(4) Voy. pl. 5, t. IV, fig. $2(p e)(o v)$. 
en dedans, on le reconnait facilement au moment de la reproduction; car de légères pressions sur l'abdomen suffisent pour produire une ponte artificielle.

Dans beaucoup des espèees que j’ai étudiées e'est dans le milieu de la longueur du sae que se trouve l'orifice. Cela s'observe nettement dans les diverses Bucardes (1), les Chames (2), les Mactres, les Pétoneles, les Pétricoles (3), les Gastrochènes, les Pholades, cte. L'orilice de la génération est toujours en dedans, et souvent plus en avant. Il y a de légères différences qui tiennent à ce que l'éloignement est plus ou moins considérable. Ainsi dans la Chame gryphoïde (4) les deux orifices sont placés tout à côté l'un de l'autre sur une ligne qui va du pied au milieu de la longueur du sac. Celui de la génération étant percé dans la masse abdominale, l'autre doit ètre tout près du bord interne de la glande.

Dans la Cardite (5) l'orifiec génital un peu saillant descend sur la face inféricure du sac de Bojanus.

Dans la Pétricole (6) le tissu de la masse abdominale accompagne III peu le canal excréteur de la génération et forme un rudiment de papille. Les rapports des orifices avec les nerfs sont dans ces deux dernières espèces extrêmement évidents.

La Bucarde rustique et les autres espèces ainsi que la Mactre des Sots présentent les deux orifiees assez éloignés; sur des individus de taille très ordinaire il $\mathrm{y}$ avait 2 millimètres de séparation. Dans ees exemples on a quelquefois de la peine à voir l'orifice du corps de Bojanus. Je pense qu’il y a un sphinerer tout autour de lui, qui par ses contractions en masque complétementl'existence. Toutefois, en tirantlégèrement à la fois d'avant en arrière et d'arrière en avant, on finit par faire entre-bâiller l'orifice qui devient alors très évident.

Enfin dans la Nloule, j’avais cru d'abord que la glande génitale. et le sae de Bojanus s'ouvraient par un mème conduit qui terminait

(1) T. IV, pl. 5, fig. 6.

(2) T. IV, pl. 5, fig. 13 (ov pe).

(3) T. IV, pl. 5, fig. 11 (ov pe).

(4) T. IV, pl. 5, fig. 13.

(5) T. 1V, pl. 5, fig. 12, Cardita sulcata (ov pe).

(6) T. IV, pl. 5, fig. 11. Petricola ruperella (ov). 
une papille fort évidente; j’ai tout dernièrement, sur des monles de forte taille que l'on apporte sur le marché de Lille, reconnu audessous de la papille et en arrière d'elle, tout à fait ì sa racine, un petit pertuis par où une injeetion a pénétré constamment et sans difficulté dans l'intérieur du sac. Ceci m'engage à mettre une certaine réserve à affirmer que dans le Lithodome dont l'organisation se rapproche à tant d'égards de celle de la Moule, ces deux organes s'ouvrent au sommet de la papille; je n'ai pas eu d'animaux des Dattiles (1) repuis mon voyage à Mahon et je ne saurais lever le doute qu'a suggéré dans mon esprit l'observation faite sur la Moule comestible.

La disposition est tout ì fait la même que l'on considère une femelle ou un mâle. Il n'y a aueune différence; on devait s'y attendre puisque déjà entre les deux glandes génitales il n'y a de différence qu'entre les parties profondes élémentaires qui sécrètent les éléments caractéristiques.

\section{§ 2.}

Circulation.

Le passage du sang au travers de l'organe qui nous oceupe a été l'objet d'une étude attentive de la part de Bojanus. C'est même la disposition du système circulatoire dans l'Anodonte qui a conduit le savant professeur de Wilna ì son opinion touchant le rôle des glandes, dont le premier il faisait l'histoire. De Blainville, tout en critiquant le travail de Bojanus, reconnut que la cireulation avait été soigneusement et exactement étudiée. Plus tard, Garner et Richard Owen indiquaient en quelrues mots les prineipaux faits, et comparaient même la cireulation de l'organe avec celle du foie. Ils montraient dans ce rapprochement que la cireulation de la veine porte est tout a fait l'analogue de celle-ci; mais e'est toujours de l'examen de une ou deux espèces que les auteurs concluent et généralisent; aussi les résultats auxquels ils arrivent doivent-ils être modifiés en certains points.

La description de Bojanus est l'expression de la vérité ; je ren-

(I) Modiola lithophaga, Lamk., $2^{\mathrm{e}}$ édit., t. VII, p. 26, n²2. 
voie done au travail de ce savant (1) pour ce qui est de l'Anodonte. Il y aura cependant quelques remarques à faire en ce qui touche les faits de structure se rapportant à la circulation.

On trouve des différences dans le mode de distribution des vaisseaux, suivant que les animaux présentent l'un ou l'autre des deux types que nous avons reconnus en étudiant la forme et la disposition des organes. Je prendrai sucessivement chacune des dispositions.

Posons en principe que le sang arrivant de l'économie pour aller respirer aux branchies doit traverser le corps qui nous oceupe; que les vaisseaux qui apportent le sang veineux sont profondément situés ou intérieurs; et que ceux, au contraire, qui le portent du sac aux branchies sont superficiels ou externes.

Ceci nous permettra d'abréger et de rendre plus simple la description.

Prenons d'abord comme exemple et comme type la Lutraire.

On sait que le sang qui arrive aux organes de la respiration après avoir traversé en tout ou partie l'organe de Bojanus, vient, d'une part, de la masse abdominale, de l'autre des lobes du manteau.

Voyons, en premier lieı, le sang des viscères ou de la masse abdominale. Dans la Lutraire comme dans l'Anodonte, la Mulette des peintres et beaucoup d'autres, on trouve en injectant, et poussant un liquide au hasard dans la masse splanchnique, un système de lacunes qui finit par se résondre en quelques veines, lesquelles, par leur réunion, donnent naissance à quelques gros trones (2) dont la fusion produit bientôt un dernier vaisseau médian (3) placé entre les deux muscles postérieurs du pied, et dirigé en arrière. Si l'on se rapporte à la description précédente de l'organe, ces quelques mots suffisent pour nettement caractériser la position de ce vaisseau. On doit, en effet, le chereher à la partie inférieure de la cloison médiane qui sépare les deux saes. Il passe au-dessous

(1) Voy. Isis, 1819, p. 46, pl. 1, fig. $1 a$; et 1820, p. 404 . Voy. l'analyse et la traduction de M. de Blainville, dans le Journal de physique, de chimie et d'histoire naturelle.

(2) T. IV, pl. 6, fig. $6(x)$.

(3) T. IV, pl. 6, fig. 6 (sm), fig. 7 (sm). 
de l'orifice de communication (1) percé dans cette cloison. C'est même au-dessous de cette ouverture que se réunissent les vaisseaux venant du foie, des glandes génitales, et du pied pour former ce canal impair que je nommerai le sinus médian inférieur.

En ouvrant ce sinus par sa face inférieure (2), on voit que ses parois sont criblées de petits orifices, surtout dans le haut. Ce sont les pertuis qui fournissent le sang à la cloison médiane, et aux parties inférieures du sac; quelques-uns sont plus grands que les autres : ils correspondent aux vaisseaux qui portent le sang plus loin dans la cloison. Le sinus médian naìt en avant et se porte vers le muscle postérieur des valves, c'est-à-dire directement en arrière. Arrivé tout près de ce muscle il se divise en quatre branches principales : deux antérieures ou collatérales, et deux postérieures ou terminales.

Les deux premières (3) se contournent en haut en se portant un peu cn dehors pour gagner l'extrémité postérieure de la partie centrale de la glande (4), de cette partie appelée poumon par Bojanus. Alors elles changent de direction, elles se portent en avant, et arrivent, en s'épuisant peu à peu, jusque vers l'extrémité antérieure de cette masse centrale. Il est facile d'injecter par le sinus médian ces branches collatérales, et de les suivre dans les parties que j'indique.

Les rameaux de terminaison(5) se portent aussi en dehors et en haut, en se contournant sur les muscles postérieurs du pied, en dedans desquels ils se trouvent. Ils gagnent la paroi postérieure et externe du sac, dans laquelle ils doivent se ramifier et apporter le sang. Après avoir parcouru ce trajet flexueux ils changent brusquement de direction pour se porter en avant, et fournir ainsi ì toutes les autres parties latérales et antérieures du sac qui ne recoivent pas de sang, des ramuscules du sinus médian, ou bien des deux vaisseaux collatéraux.

(1) T. IV, pl. 6, fig. 6 (z).

(2) T. IV, pl. 6, fig. 7 (sm).

(3) Pl. 6, fig. 6 et fig. 7 (i).

(4) Pl. 6, fig. 6 el fig. $7(p)$.

(5) Pl. 6, fig. $6(l)$. 
Il est à peine utile d'ajouter que ces quatre vaisseaux se distribuent symétriquement deux de chaque côté du corps.

Ainsi voilà un vaisseau médian, résultat de la réunion des troncs veineux arrivant de la masse abdominale, qui se ramilie tout à fait il la manière des artères dans le tissu du corps de Bojanus; c'est une véritable circulation de la veine porte. Il n'y a pas seulement ressemblance quant à l'origine et à la division des vaisseaux, mais eneore ce système circulatoire est placé sur le trajet du sang, qui des viseères va aux organes de la respiration, e'est-à-dire sur le trajet du sang veineux qui revient des viscères chargé de la matière alimentaire absorbée après la digestion, absolument comme dans les animaux supérieurs. A ne considérer done que la circulation, il n'est pas douteux que l'on ne trouve ici l'analogive du foie; mais je reviendrai sur ce fait en parlant des fonctions de la glande. Je veux toutefois bien établir ce fait que les Mollusques présentent une petite circulation analogue à celle du foie des animaux supérieurs, avec cette différence qu'elle se passe dans un organe tout différent de ce dernier.

Après s'être ramifiés dans le tissu du corps glandulaire les vaisseaux se résolvent en capillaires, dont je n’étudierai point ici la disposition, renvoyant pour cela au moment où je m'occuperai de la structure.

Voyons maintenant les vaisseaux efférents. Ceux-ei reçoivent le sang des capillaires, et le portent aux branchies. Il y a bien une disposition générale et constante dans leur position, mais il se présente de nombreuses et très grandes variations dans la forme et le nombre : la position seule est constante. Si les vaisseaux afférents étaient profondément placés, les vaisseaux efférents, au contraire, sont toujours en dehors à la surface externe de l'organe; c'est eux que l'on aperçoit d'abord quand on réussit bien les injections, ou bien quand, sur la paroi externe de l'organe, il s'en dessine sans préparation.

Dans les Mulettes, les Anodontes, il y a des vaisseaux distincts portant le sang de l'organe aux branchies en avant et en arrière, ainsi qu'au feuillet interne et au feuillet externe : Bojanus les a exactement indiqués; mais dlans la Lutraire qui nous occupe 
il en est autrement : les rameanx externes couvrent d'un lacis très épais la paroi du sac, et débouchent, dans des sinus creusés dans la substance glandulaire, mais toujours placés en dehors. Ces sinus, quand on réussit bien à les injecter, font saillie sur le plancher du péricarde ou sur les parois latérales. En les ouvrant (1), on voit les pertuis nombreux qui apportent le sang dans leur intérieur.

Tous ces sinus convergent vers un point latéral et symétrique placé de chaque côte dans la partie la plus dilatée transversalement $(2)$ de l'organe. C'est là que viennent aboutir tous les vaisseaux branchiaux.

On doit reconnaitre quelques sinus principaux, qui, dans la Lutraire, offrent une disposition constante. Il en est un long (je ne décris qu'une côte puisque la disposition est symétrique), qui, de l'insertion branchiale à l'extrémité postérieure de l'organe, se dirige d'avant en arrière jusque dans le prolongement de la glande qui remonte sur le musele postérieur des valves. Sur son bord interne, un peu au delà du milieu de la largeur en arrière, on voit un sinus plus petit, secondaire, qui se porte en dedans. Tandis que le premier mérite le nom de sinus latéral (3), celui-ci peut être appelé sinus dorsal (4).

L'extrémité antéricure dı sinus latéral est fort dilatée et large; quand on en enlève la paroi externe, on distingue, sur la paroi profonde en dehors, du côté de l'insertion des branchies, une série linéaire de pertuis dont les deux derniers, en avant et en arrière, sont assez grands pour permettre l'introduction d'une tête d'épingle. Ces orifices ne sont autre chose que les bouches des vaisseaux branchiaux, qui recoivent le sang accumulé dans les larges sinus creusés dans la substance même de la glande.

Vers l'extrémité postérieure du sinus latéral, on en trouve un autre qui est plus inférieur que celıi-ci : il mériterait le nom de

(1) T. IV, pl. 6, fig. 4 et $5(m n q)$.

(2) T. IV, pl. 6, fig. 4, 5 ( $\mathrm{m} \mathrm{m})$.

(3) T. IV, pl. 6, fig. 4 et $5(q$ et 0$)$.

(4) T. IV, pl. 6 , fig. 4 et $5(\iota)$. 
sinus inférieur (1). Son extrémité postérieure se conburne en dedans, plonge dans la substance glandulaire pour aller recueillir le sang cui a traversé la partie centrale de l'organe, le poumon de Bojanus (2).

Ansi, en résumé, le sang est porté prơondément dans tout l'organe par les ramifications dı vaisseau médian. Il est recueilli par les capillaires, qui le déversent dans les sinus superficiels et externes, d'où il va, par la réunion en mu mème point de tous exs sinus, dans l'appareil de la respiration.

Nous venons d'indiquer la marehe que suit le sang apporté de la masse viscérale; mais il ne vient pas tout de ees parties, il ne passe pas tout par la glande.

II. Milne Edwards, dans ses beaux travaux sur la circulation des Mollusques, a montré qu'une partie du sang du manteau arrivait directement dans les oreillettes sans passer par l'appareil de la respiration. Eh bien, une disposition analogue se présente pour li circulation, que nous éludions en ce moment. On trouve en avant et en arrière, mais surtout en haut et en bas du musele des valves, un lacis de canaux, à parois aussi vagues et aussi peu limitees que. dans le reste de l'organisme, qui recoit postérieurement dı sang des parties qui l'avoisinent, et qui le verse antérieurement en partie dans les tissus spongieux de la glande, en partic dans les branchies. Dans la Lutraire le mantean est très développé en arrière, et reçoit une quantité de sang assez grande pour rendre turgides les tubes respirateurs; une grande partic du liquide, en revenant de ces parties, tombe dans ce sinus latéral ou inférieur, et se rend directement aux branchies sans traverser par conséquent l'organe de Bojanus.

Mais cette particularité est loin d'être aussi nettement tranchée que dans les animaux que nous allons étudier maintenant. Si, dans. la eoquille de Saint-Jaeques, les choses se passent un peu difléremment, toujours néanmoins les vaisseaux afférents sout profonds, toujours les vaisseaux eflérents sont superficiels.

On sait que dans les Peeten dont il s'agit la masse viscrírale as

1) T. IV, $\mathrm{pl}, 6$, fig. \& et $5(n)$.

(2) T. IV, pl. 6, fig. b et $7(p)$. 
divisée presque en deux moitiés : l'une, antéro-supérieure, composée plus exclusivement du foie; l'autre postérieure, que j’ai comparée à la bosse de polichinelle, formée par les glandes génitales; entre les deux, est un étranglement où l'on voit le pied. Cette sorte de division des viscères conduit à une division analogue des vaisseaux; il faut donc éludier la marche du sang qui vient du foie et de celui qui vient des organes génitaux.

En poussant le liquide par les lacunes périjécorales, il est facile d'injecter les vaisseaux veineux (1), qui se ramilient à la manière habituelle au milieu des lobules du foie. On voit alors qu'ils se réunissent en trones plus ou moins constants, qui toujours finissent par former un vaisseau distinct; celui-ci n'est plus impair et médian, mais il est double et symétrique, on le retrouve de chaque côté(2); en sorte qu'en définitive, tout le sang arrivant du foie est. contenu dans deux vaisseaux, qui se dirigent du haut en bas et d'avant en arrière en passant sur les côtes de la bouche, et gagnant les côtes de la base du pied. Comme e'est au niveau à peu près de la bouche qu'a lieu la communication entre les deux glandes, le vaisseau dont il est ici question se trouve done près de cette communication exactement entre elle et la bouche.

Ce vaisseau afférent ne plonge dans la substance glandulaire qu'après avoir dépassé la base du pied, et être arrivé dans la partie vraiment parenchymateuse: il y pénètre de dedans en dehors, et il faut alors, pour pouvoir continuer à le suivre, ouvrir le sac.

Dans l'Huitre vermeille (3), quand on ouvre la poche avant d'avoir étudié la circulation, on est frappé par l'apparence vasculaire que présente son intérieur. On voit naître en face de l'espace qui sépare la masse abdominale de la masse hépatique, un faisceau qui se jorte directement en dehors et en haut, en émettant de chaque côté des rameaux dont les anastomoses forment un lacis, qui laisse entre ses mailles des dépressions nombreuses donnant à la eavité et a l'organe tout entier l'aspect d'un tissu spongieux. $\Lambda$ la plremière

(1) T. IV, pl. 6, fig. $1(j)$.

(2) T. IV, pl. 6, fig. $1(y)$.

(3) Spondylus gaderopus. 
vue, on reconnait dans le Spondylus goederopus (1) la richesse vasculaire du corps de Bojanus.

Dans le Pecten $(2)$ une disposition ì peu près semblable se présente aussi; mais les vaisseaux sanguins venant du foie sont plus difficiles à suivre, il est nécessaire de les injecter, ce qui permet de voir que le vaisseau dont je parlais il n'y a qu'un instant se divise en deux branches, dont les subdivisions secondaires s'entrecroisant dans tous les sens forment un lacis, véritable réseau d'où résulte un tissu spongieux. Les deux branches cheminent à la face interne : l'une est plus supérieure que l'autre. On remarque dans cette différence de la distribution des canaux sanguins quelque chose de correspondant à ce que nous avons vu dans la disposition générale de l’organe.

Le sang qui revient de la masse abdominale aux organes de la reproduction, chemine dans des vaisseaux très faciles à distinguer sans aucune préparation $(3)$. Ils sont arborescents et assez régulièrement disposés; au premier abord on est disposé à les prendre pour des artères. Leur nature ne peut faire l'ombre d'un doute; car en poussant une injection par le cœur, on n'arrive à les injecter qu'après avoir fait des ruptures, à moins que l'injection n'ait tellement bien réussi que, les tissus se colorant d'une manière générale, le liquide ne passe par les lacunes des artères dans les veines. I'ailleurs ils aboutissent par leur grosse extrémité à l'organe de Bojanus; ils ne peuvent done pas appartenir au système artériel, Ces vaisseaux se portent en dedans vers l'organe, et s'introduisent dans sa substance profondément du côté des vaisseaux venant du foie.

Ainsi voilà deux voies différentes par où le sang arrive du corps à l'organe. Dans l'Anodonte, la Lutraire, un seul vaisseau médian impair distribue ses ramifications à tout l'organe; ici, au contraire, des vaisseaux de deux ordres pour chaque côté, et portant chacun le sang d'une partie spéciale.

(4) T. IV, pl. 4, fig. 6 ( $r r$.

(2) T. VI, pl. 6, fig. 1. Le sac de la glande a été ouvert, et les parois étalées permettent de voir le lacis des vaisseaux entourant les deux troncs principaux.

(3) T. IV, pl. 6. lig. 2 (v.). 
Les vaisseaux efférents (1) sont faciles à voir, même sans injection, dans les coquilles de Saint-Jaeques. Ils ne sont plus semblables aux sinus de la Lutraire, et ils ressemblent absolument aux vaisseaux veineux que l'on remarque sur la face externe de l'abdomen. On en voit sans préparation les arborisations dont les ramuscules les plus déliés sont tournés ver's le bord interne, tandis que les gros trones se portent sur la face inférieure vers le point où s'insèrent les branchies, et s'ouvrent directement dans le sinus branchial (2).

Nous avons vu que daus la Lutraire, tout le sang qui allait aux branchies ne traversait pas l'organe de Bojanus. Il en est de même ici, mais la chose est plus nettement caractérisée. Au-dessous dı muscle des valves, si développé dans l'espèce que nous étudions, on trouve un lacis de vaisseaux qu'on injecte avec la plus grande facilite, et qui communique avec deux larges poches (3) piriformes, véritables sinus, placées sous l'altache de ce repli falciforme, qui sert de base d'insertion aux branchies. Ces deux sinus, que l'on in. jecte avec la plus grande facilité, reçoivent aussi du sang qui revient du muscle des valves (4), et de quelques autres parties postérieures du manteau. En avant ils s'allongent et s'effilent en un véritable col, pour s'aboucher avec la base du vaisseau branchial dans un point tout voisin de celui où les vaisseaux efférents de l'organe de Bojanus viemnent eux-mêmes s'ouvrir. Il est facile de remplir d'injection à la fois les vaisseaux du corps de Bojanus et ces sinus en poussant le liquide coloré par le vaisseau branchial, qui court le long du bord concave de la branchie.

On voit qu'au fond la circulation se passe dans le Peigne comme dans la Lutraire; et que les différences que nous signalons tiennent simplement à des modifications dans la forme de la glande.

Dans la Moule comestible ainsi que dans l'Anomie, la glande génitale occupe en grande partic l'épaisseur du manteau. Le sang qui revient de ce dernier est en quantité très considérable, on le comprend; il un traverse pas tout le corps de Bojanus. Il y a, en
(1) T. IV, pl, 6, lig. $2(h)$
(2) T IV, pl. 6, fig. $2(s h)$
(3) I IV, pl 6, fig. $2(s s)$
(4) T. IV, pl. 6, fig, I (s). 
ellet, des vaisseaux qui sont jetés comme des ponts au-dessus de la glande, surtout en dehors, entre le manteau et la veine branchiale, d'où résultent ces lamelles séparées par des sillons, ce qui a fait croire à Siebold que la glande était ouverte dans tonte sa largeur.

En résumé, on voit que le sang traverse en grande partie, avant d'aller s'hématoser, la glande de Bojanus; mais que, s’il ne la traverse pas en totalité, cependant celui qui revient des organes de la digestion ne fait en aucun cas exception. Il ne peut done être douteux qu'il ne se passe dans la glande quelque action physiologique importante.

A part l'étude faite par Bojanus sur la Moule des étangs, peu de travaux traitent d'une manière suivie la partie de la circulation dont je viens de faire l'histoire. Cependant M. Deshayes s'en est oceupé dans les différentes monographies qu'il a publiées dans l'Exploration scientifique de l'Algérie. La circulation, telle que la décrit le savant conchyliologiste dans l'organe dépurateur (c'est ainsi qu'il nomme le corps de Bojanus), n'est pas, si j'ai bien compris et interprété le texte de la publication, en rapport avec les faits que je viens de rapporter.

M. Deshayes, après avoir dit, en s'appuyant sur l'opinion de quelques zoologistes, que l'organe dépurateur a de larges communications avec le système veineux général, “... que tout le sang » Sans exception devait passer à travers ses cavités avant de ren" trer dans le système aortique, » déclare que ce fait est pour lui indubitablement établi (1). Dans de très belles figures, il montre la connexion avec le système veineux général.

Mais il devient difficile de le suivre dans les détails qu'il donne à propos de la communication de l'organe dépurateur avec l'oreillette. Je citerai le passage. Au sujet des Pholades, il dit (2) : "L'extrémité postérieure de l'oreillette a des connexions avec l'or" gane dépurateur, et c'est là qu'il faut chercher la communication » qui existe entre ces deux organes. Dans le Solen siliqua, l'extré" mité des deux piliers rharnus vient se terminer en pointe aiguë

(1) Loc. cit, , p. 167.

(2) Loc. cit., p. 167, 18, fig. \& $(m n)$ : fig. $3(d e)$. 
" à l'angle antérieur de la cavité de l'organe (pl. 18, fig. $4 \mathrm{~m}, n$, » fig. $3 d, e$ ), et c'est en suivant la direction de ces deux piliers que "l'on arrive à la petite ouverture communiquant avec l'oreillette. »Cette ouverture est ovale-oblongue (pl. 18, fig. $3 f$ ); elle est "garnie de chaque côté d'un petit pilier un peu plus épais, qui, "probablement, remplit les fonctions d'une valvule. "

Il est difficile de savoir si l'auteur, quand il parle de communications, a entendu désigner la cavité, le sac lui-même, ou les vaisseaux de ses parois. On ne peut guère admettre que ce soit la cavité du sae qui entrerait en communication avec l'oreillette; cependant la description de l'orifice ayant deux piliers charnus servant de valvules semblerait l'indiquer.

Dans tous les cas, en admettant que l'oreillette soit en communication avec le tissu seul de l'organe, il y a là quelque chose que je n'ai point observé. J'ai montré dans ce qui précède que l'organe de Bojanus était placé sur le trajet du sang entre le corps et les branchies; que le sang qui s'échappait de ses vaisseaux allait s'oxygéner dans les organes de la respiration. Il ne peut done arriver aux oreillettes qu'après avoir traversé les branchies. Peut-être y a-t-il quelques vaisseaux qui, du corps de Bojanus, vont directement aux oreillettes, comme cela se voit pour le manteau; mais je n'ai pas souvenance d'avoir jamais rempli les oreillettes en poussant mes injections dans les tissus de l'organe, et cependant bien souvent elles ont assez bien réussi pour remplir complétement les vaisseaux branchiaux. Toutefois je dois dire que M. Deshayes n'est pas seul de son opinion. Bojanus a décrit dans l'Anodonte des vaisseaux qui vont aussi à l'oreillette. Il le dit en plusieurs endroits. Il appelle artères du réservoir des œufs les vaisseaux qui vont aux branchies, et veines ceux qui se rendent à l'oreillette. Le sang suivrait done deux trajets à la sortie de l'organe pour revenir au cœur. Il irait, d'une part, directement, de l'autre indirectement en traversant les branchies. Je puis dire en tous cas que la première communication est secondaire, mais on comprend que Bojanus ait dù lui attribuer une grande importance en raison même de son opinion, puisque les branchies n'étaient pas pour lui l'organe de la respiration, et que le corps glandulaire était le joumon; dans 
celle manière de voir, il élait naturel de ne pas admettre que tout le sang ayant respiré passît par le réservoir des œufs (branchies).

Ce qui me porterait à eroire que la circulation n'a pas parfaitement été entrevue par M. Deshayes, c'est qu'il arrive à cet auteur d'injecter le tissu de l'organe dépurateur par l'arlère postérieure.

“ Si l'on parvient à injecter l'aorte postérieure, ce qui est quel" qutefois très difficile, on voit alors les diverses surfaces de l'or"gane.... se couvrir d'un réseau vasculaire très riche, dont les " rameaux communiquent entre eux par une multitude d'anasto"moses. Lorsqu'on a l'organe ainsi injecté, la couleur de son tissu » est tellement changée qu'il faut un examen attentif, et des gros" sissements suffisants pour reconnaître que ce cliangement n'est " pas dù à une simple imbibition des tissus, mais à une véritable " injection. "

D'après cela il y aurait un troisième ordre de vaisseaux aussi développé que les précédents, et qui porteraient du sang artériel. Je dois dire n'avoir rien vu de semblable, et l'aveu d'une grande diffieulté à parvenir à injecter par l'aorte postérieure ferait eroire qu'il y a eu quelques ruptures dans les cas de réussite.

Richard Owen (1) et Garner (2) font remarquer que le sang traverse l'organe pour arriver aux branchies, mais ils ne donnent pas de détails.

\section{$\S 3$.}

Structure et Texture.

La structure et la texture de la glande sont faciles ì reconuaître, et si l'on sait peu de chose à leur égard, cela tient tout simplement ì ce que les auteurs ne s'en sont occupés que secondairement et aceidentellement, pour ainsi dire.

M. von Siebold en parle ainsi : " Le parenchyme des parois " de ces sacs est formé par un tissu lâche, qui, à la moindre "lésion, se décompose en petits corpuscules vésiculeux et gra" nuleux. La plupart de ces corpuscules contiennent un noyau

(1) Richard Owen, Lectures on comparative analomy, vol. I, p. 284.

(2) Garner, The magazine of nutural history by Charlesworlh, p. 167, 1839. 
"arrondi, d'un noir bleuitre, auquel est due la couleur plus on » moins foncée des reins, etc. » Et il ajoute en note : "La struc„ ture intime de ces organes n'a pas assez attiré l'attention jus» qu'ici (1). »

Rien n'est facile à obtenir comme l'élément anatomique dont il sagit; en effet, il suffit de prendre au hasard une portion de la glande, et de la porter sous l'objectif du mieroscope pour voir dans le liquide flotter une multitude de cellules facilement reconnaissables, et dont le volume, quoique variable, est cependant presque toujour's assez considérable. Dans quelques exemples, le tissu semble s'égrener, et les corpuscules qui le composent, devenus libres el gonflés par l'endosmose, paraissent très transparents et parfaitement sphériques. Cela s'observe avee une grande facilité dans lit Chama griphoïdes (2), le Corbula striata (3), la Spondylus Gaderopus (4), la Lima squamosa (5), la Lucina lactaca (6), etc., el beaucoup d'autres dont je n'ai pu donner le dessin.

Lorsque l'endosmose a ainsi gonflé les petites utricules, leur contenu se montre plus nettement, ear il semble s'être limité davantage, et ramassé en une petite masse autour d'un noyau; celui-ci présente dans son intérieur des nucléoles, ou tout au moins ces petits corpuscules que l'on est convenu d'appeler ainsi.

La cellule a, du reste, des apparences très variables : quelquefois elle est complétement transparente et vide de tout corpuseule autre que ce noyau, comme on le voit dans la Pandora rostrata $(7)$. On peut presque poser cette règle : plus le noyau est bien dessinć, nettement limité, et moins le contenu de la cellule est abondant. Ainsi dans la Pandore, le noyau est seul au milieu de la cellule ; il en est à peu près de même dans la Chame $(\mathrm{S})$, llans la Telline, la

(1) Anatomie comparée, trad. franç., t. I, $2{ }^{\circ}$ part., p. 279 et note 3

(2) T. IV, pl. 4, fig. 10

(3) T. IV, pl. 4, fig. 3.

(4) T. IV, pl. 4 , fig. 7 et 8 .

(5) T. IV, pl. 4, fig. 2.

(6) T. IV, pl. 4, fig. 11 et 12

(7) T. IV, pl. 5, fig. 15.

(8) T. IV, pl. 4, fig. 10 
Lutraire (quand il n'y a pas une cristallisation), ainsi que dans la Pholade où j’ai observé aussi quelque chose de fort analogue.

Mais il faut l'avouer, les choses varient avec l'époque à laquelle on observe, et souvent dans un même individu une partie de la glande a des cellules dont les noyaux sont très distincts et le contenu nul, tandis qu'ì côté une autre partie a les cellules presque remplies de granulations épaisses et serrées.

Toutefois, dans la Dattile (1) des Mahonais, j’ai presque constamment observé que le noyau n’était pas aussi distinct, et que la cellule était le plus souvent, je pourrais même dire toujours, remplie de granulations colorées, qui donnent au tissu son apparence particulière.

Dans le Pectunculus pilosus (2), j’ai observé quatre, cinq petits noyaux, et quelquefois plus, qui n'étaient pas toujours rapprochés.

Du reste, cette différence du contenu peut donner diverses apparences au tissu, comme on le verra plus loin.

Les cellules sont de taille très variable; dans la Pholade, la Pétricole, elles m'ont paru relativement fort petites. Dans cette dernière, le noyau est fort petit, peu considérable, très limité, et le contenu nul (3). Dans l'Huître vermeille, la Prère ou Corbule, elles acquièrent un développement plus considérable (4).

J'ai dessiné le tissu de la glande de la Chama griphoüdes dans la planche 4, figure 10, et l'on voit que sur une même espèce, un même individu et une même partie, il y a une grande différence dans le volume des éléments.

Les dimensions sont déjà plus considérables dans la Lime (5); mais e'est surtout dans la Telline et la Lucine (6) que l'on observe les cellules de la plus grande taille.

Dans quelques cas peu nombreux, il m'a paru y avoir de petites cellules enfermées dans des cellules plus grandes; on dirait une pro-

(1) T IV, pl. 5, fig. 7 et 8 .

(2) T. IV, pl. 5, fig. 16.

(3) T. IV, pl. 5, fig. $1(a)$.

(4) T. IV, pl. 4, fig. 3,8 .

(5) T. IV, pl. 4, fig. 2.

(6) T. IV, pl. 5, fig. $10(b c)$. 
duction endogène; c'est ce qui s'est offert avec évidence dans le Pectunculus pilosus (1). Bien que, dans cet exemple, ce mode de développement ne puisse être révoqué en doute, jen'oserais cependant affirmer qu'il existe toujours, sans exception, car je ne l'ai pas retrouvé dans tous les cas.

Il faut aussi indiquer ce qui se montre d'une manière à peu près constante dans les Lucines (2), ainsi que dans quelques autres espèces. Le noyau dont je reparlerai plus loin est nettement circonscrit ; il est volumineux, et fortement accusé par sa teinte brunâtre. La cellule qui l'enferme est grande, et ses bords sont bien limités; mais en observant attentivement et faisant varier les inclinaisons du miroir du microscope, on distingue comme un léger contour qui entoure le noyau en dedans de la cellule; il y aurait presque l'apparence d'une cellule plus petite, incluse dans la plus grande. Certainement ceci condıirait à admettre un développement de la cellule autour du noyau comme Schwan l'a indiqué; mais la même apparence se présente, alors qu'il y a deux ou trois noyaux.

Je ne pourrais dire si cette apparence est due à la présence d'une substance, de puissance réfringente différente, entourant le corpuscule central, ou si elle est la conséquence de l'inclusion d'une cellule.

Dans une observation que malheureusement je n'ai pu répéter, les Lucines étant fort difficiles à trouver, quoique très abondantes sur les côtes de Bretagne, j'ai cru voir autour d'un noyau considérable, occupant une grande partie de la cellule, un cercle pellucide, entouré lui-même d'une zone obscure, où des granules très fins et peu développés se mouvaient d'un mouvement brownien. Je le répète, y a-t-il une substance hyaline entourant le noyau? y a-t-il deux cellules emboîtées? C'est ce que je ne puis décider.

Ainsi l'élément microscopique de l'organe est une cellule enfermant une matière brunâtre plus ou moins jaune verdâtre, tantôt éparse, tantôt limitée, et formant un noyau qui cause la coloration générale de la glande.

(1) Voy. t. IV, pl. 5, fig. 16.

(2) Voy. t. IV, pl. 4, fig. $12(a b c)$. 
Le contenu offre encore quelques autres particularités; mais je renvoie pour les faire connaître au moment où je m'occuperai des fonetions.

Comment ces éléments se réunissent-ils, et forment-ils le tissu de la glande? C'est ce qui nous reste à étudier.

Les cellules s'accolent les unes aux autres assez lâchement, car clles ne forment jamais un tissu dense et résistant, se compriment et deviennent polyédriques; ce qui fait que lorsque l'on a dı tissu sur les yeux, on croirait avoir parfois affaire à du tissu végétal. Dans quelques Mactres des côtes de Bretagne et de Normandie, quand la matière colorante n'est pas encore bien développée, l'illusion pourrait être complète pour un observateur qui ne connaitrait point l'origine de la préparation; les cellules sont réunies par couches, dont l'épaisseur est mesurée par trois, quatre et même davantage.

Ces couches de cellules, lâchement unies, tapissent la paroi interne du sac. Les éléments les plus externes, ceux qui limitent la substance glandulaire, sont hérissés de cils vibratiles, qui acquièrent souvent une grande largeur. Dans les Mactres surtout, les cils deviennent de longs filaments flabelliformes. Dansles Pandores(1), ils atteignent aussi un grand développement. Ici l'épithélium est formé par la substance elle-même ; car, à part les cils vibratiles, il n'y a aucune différence entre la cellule la plus externe et les deux ou trois qui la suivent.

Dans l'Arche de Noé, au contraire, les cils sont fort peu allongés, et ils forment comme un fin duvet mobile à la surface interne du sac.

Je dois appeler l'attention sur deux exemples, où les cellules grandes, bien développées, nucléolées, ne sont pas les plus externes. La couche vibratile est formée de cellules ou corpuscules assez petits qui, depuis la surface libre jusqu'aux parties profondes, augmentent de volume. Le Spondyle (2) et la Corbule (3) offrent cette disposition, qui m'a paru évidemment exceptionnelle. Y aurait-

(1) Voy. t. IV, pl. 5, fig. 15.

(2) Voy. t. IV, pl. 4, fig. 7.

(3) Voy, t. IV, pl. 4, fig. 3. 
il cu une erreur dans mon observation? A côté d'une partie de tissu peu développée, serait-il venu se placer un tissu dont les cellules avaient acquis toutes leurs proportions?

Dans son ensemble, le tissu peut offrir deux aspects différents : tantôt la séparation des cellules est marquée par un trait (1) obscur, tantôt au contraire par une bande plus claire (2). Le premier cas a lieu quand le contenu des cellules est réuni en une petite masse bien distincte, en un noyau, ou bien quand la cellule est vide et transparente ; le second se présente quand les granulations sont très nombreuses, la cellule paraissant alors entièrement obscure, et les points de contact, les parois accolées des diverses cellules, produisant une petite bande transparente. C'est quelque chose de tout à fait semblable que l'on observe, lorsque le tissu végétal est gorgé de chlorophylle. Cette apparence s'est présentée assez habituellement dans les Dattiles ou Lithodomes du port de Mahon.

Quand on ouvre le sac de Bojanus, on voit que la surface de la cavité est comme veloutée ou tapissée de sortes de papilles. Le dessin d'une portion grossie de la glande du Spondyle (3) que je donne est une représentation exacte de l'apparence de la face interne de la glande. Nous devons chercher maintenant qu'est-ce qui produit ces inégalités.

Si l'on soumet à l'examen microseopique, mais à un grossissement faible, une portion assez considérable du tissu de la glande, non plus pour en connaitre les éléments, mais pour en étudier la disposition générale, on remarque, en comprimant légèrement, que les saillies dont il vient d'être question sont loin d'être pleines, qu'à leur centre est une cavité ; et si l'observation que j'indique est faite sur la glande d'un animal injecté, on voit que la matière colorante occupe cette cavité.

En employant un grossissement un peu plus fort, on ne tarde pas à s'apercevoir qu'autour de cette cavité, véritable dépendance du système sanguin, est une couche mince et régulière de tissus

(1) T. IV, pl. 5, fig. 7 port. (b).

(2) T. IV, pl. ๖̌, fig. 7 port. (a).

(3) T. IV, pl, 4, fig. 9. 
glandulaires; de telle sorte que l'on peut considérer chacune de ces élévations comme un prolongement en cul-de-sac de l'arbre circulatoire, entouré par de la substance glandulaire. Il y a donc iei quelque chose qui rappelle un peu, mais avee beaucoup de simplicité, ce que l'on voit dans les villosités intestinales, sauf, bien entendu, les fonctions auxquelles je ne veux nullement faire allusion.

On peut dans l'étude du corps de Bojanus se représenter dans toute sa simplicité la marche de la sécrétion; le vaisseau capillaire, qui forme une anse dans la petite élévation papilleuse du sac, entouré par un parenchyme capable de prendre, sans doute, par endosmose les éléments de la sécrétion, fournit au tissu le liquide qui sert à élaborer les produits; et la substance sécrétante est, on le voit, aussi rapprochée que possible du sang.

Ici se présente une question importante, la substance cellulaire de cette couche que nous venons de décrire est-elle en dehorsd'une paroi propre au vaisseau, ou bien forme-t-elle la paroi même du vaisseau? En d'autres termes, les capillaires que l'on injecte dans la paroi de la glande sont-ils distincts de la substance, ou bien sontils des lacunes ereusées dans cette substance? Je dois avouer que, dans bien des cas, dans la Pinne marine (1), dans la Pétricole (2), la Bucarde (3) rustique, il semble difficile de ne pas admettre cette dernière manière de voir, car la couche cellulaire est épaisse simplement de deux ou trois cellules, et l'on distingue très nettement la cavité centrale, surtout dans la Pinna nobilis et la P'etricola ruperella.

I a-t-il une pellicule mince, anhysste, qui limite cette cavité, et tapisse en dedans les contours du parenchyme glandulaire? Cela est possible, mais la démonstration en est bien difficile, sinon impossible.

Que si l'on admet la première des opinions, on voit encore ici plus nettement le rapport de la substance glandulaire sécrétante avec le liquide sanguin; et l'on peut presque dire que la substance

(1) Voy. t. IV, pl. 5, fig. 9

(2) Voy t. IV, pl. 5, fig. I.

(3) Voy, t. IT, pl. "̈, fig. i 
puise par un de ses côtés les matériaux qu'elle sécrète et verse au dehors par l'autre.

Si maintenant on rapproche de ces descriptions la théorie moderne des sécrétions, qui explique la production des liquides par une muc et une déhiscence incessantes des cellules du parenchyme, on voit que le mouvement dans la production des cellules doit se passer du dedans au dehors, c'est-à-dire de la partie en contact avec le liquide sanguin à celle que couvrent les cils vibratiles. On pourrait toutefois objecter à cette manière de voir les deux exemples que je présentais il n’y a qu'un instant, dans lequel les cellules les plus développées n'étaient point celles qui avoisinaient les cils; mais on se rappelle quelles restrictions j'ai cru devoir faire.

On a vu que quelques faits semblaient démontrer le développement endogène des cellules. Dans la Tellina solidulaque je pêchais aux Hébiens, j’ai remarqué qu'entre les cellules fort grandes et renfermant des noyaux fort développés, il y avait de petits points d'une matière jaunâtre analogue ì celle qui forme le noyau des cellules; et je me demande si ces granulations ne sont pas destinées à devenir le noyau de cellules futures. Ici le développement ne serait plus endogène.

Enfin il est une disposition que je ne dois pas passer sous silence, car elle peut, au premier abord, paraître embarrassante. Le tissu glandulaire de la Bucarde rustique (1), observé à un assez faible grossissement, paraît formé de petits prolongements creusés d'un eul-de-sac; mais les cils vibratiles, au lieu d'être en dehors de ces prolongements, comme cela a lieu dans les autres espèces, se trouvent en dedans. On ne peut arlmettre que les vaisseaux sanguins soient ici dans le centre du prolongement lì où paraissent les mouvements eiliaires. En observant le tissu de la glande de la Pétricole, on remarque (2) alternativement des dépressions et des saillies de la surface, on observe une même chose dans la Modiole lithophage (3). Aussi dans ces exemples trouve-t-on tous les pas-

(1) Voy. pl. 5, fig. 4.

(2) Voy. t. IV, pl. 6, fig. 1

(3) Voy. I. IV, Il. "5, fig. 7 
sages entre les lobules saillants de la Pinna nobilis (1), et les culsde-sac du Cardium rusticum (2).

En résumé, que l’on considère les vaisseaux sanguins comme élant distinets, et formant un lacis considérable recouvert d'une couche de substance cellulaire de nature glandulaire, ou bien que l'on admetle une couche glandulaire onduleuse dans les replis de laquelle sont creusés des eanaux où s'épanche le sang dans son trajet du corps aux branchies, toujours aura-t-on une idée exacte de la disposition et de la structure de la glande fort simple, en définitive, commie on le voit.

III.

Rôle physiologique du corps de Bojanus.

C'est en faisant toute réserve que je présenterai quelques considérations sur la physiologie de cet organe; je l'ai dit en conmençant, il est nécessaire d'entreprendre quelques recherches nouvelles; je ne puis cependant à la fin de ce travail, presque entièrement anatomique, m'empêcher de faire connaitre les faits qui me paraissent résulter des observations nombreuses que j’ai pu et dù faire, tant pour étudier l'organe lui-même que pour faire l'histoire des organes de la reproduction des Acéphales. Je regarde, du reste, les considérations qui suivent comme destinées à servir de renseignements pour un autre travail. Ce travail, je l'espère du moins, s'accomplira plus tard, et je pourrai alors remplir la lacune que présente aujourd'hui mon mémoire.

Il est utile d'abord de bien établir quelles opinions ont été successivement soutenues par les auteurs; il est peu d'organes dont le rôle ait été aussi diversement interprété. Bojanus (3) est le premier auteur qui ait séricusement entrepiris d'en faire connaitre l'histoire. Dans un mémoire fort remarquable sur la respiration de

(1) Voy. t. IV, pl. 5, fig. 9.

(2) Voy. pl. 5. fig. 4.

(3) Voy. loc. cit., traduction de Blainville, et le mémoire original dans l'Isis, 1819. 
l'Anodonte, cet auteur cherche ì prouver que le sac glandulairedes Acéphales est un poumon. Celte opinion n'est plus soutenable et soutenue aujourd'hui; cherchons cependant comment Bojanus avait été conduit à cette manière de voir qui, du reste, n'était pas nouvelle, comme l'observe avec raison de Blainville, puisque Méry l'aviit déjà émise à l'Académie des sciences de Paris.

Bojanus n'observant que l'Anodonte (Anodon cygnæum), et voyant toujours l'animal porter ses petits dans les feuillets de ses branchies, en conclut que ces organes étaient des matrices on réservoirs dles ceufs. Dépossédant ainsi les feuillets branchiaux d'un rôle qui leur avait été jusqu'alors attribué, il lui fallait trouver ailleurs les organes de la respiration.

En faisant des injections, il reeonnut bien vite la richesse vasculaire des glandes brunîtres placées de chaque côté du corps; il supposa que cette richesse devait être en rapport avee une fonetion importante : trouvant un sac ouvert à l'extéricur, dans la large cavité durquel s'élevait une éminence, qu’il crut spécialement vasculaire, il fut conduit à admettre que le sac était la cavité respiratoire où entrait l'eau; que la masse saillante était le poumon, organe même de la respiration.

S'il n’y avait là rien de nouveau, puisque déjà Méry avait eu cette opinion, il y avait an moins quelpue chose d'incorrect et l'étrange. Quelque chose d'incorrect, car, ainsi que n'a pas manqué de le dire M. de Blainville, il n'y a pas de poumon quand la respiration est aquatique; et quelque chose d'étrange, parce que tout le sang ayant respiré, tout le sang artériel aurait, avant d'arriver au corps, traversé un organe où la vitalité doit être très active au moment de la gestation.

Ce qu'il y a d'exact dans le travail de Bojanus, c'est la description de la circulation. Il semble même que la circulation lacunaire y est entrevue; mais comme le savant professeur de Wilna ne faisait ses études que sur une seule espèce, il ne devait el ne pouvait généraliser ; aujourd'hui que les beaux travaux de M. Milne Edwards nous ont fait connaitre complétement cette disposition de la circulation particulière aux Mullusques, nous pouvons dire que Bojanus avait vu la disposition, sans se rendre un conpte bien exact de ce qu'il 
voyait. Voiei ec qu'il dit; jemprunte te passage à la traduction donnée par M. de Blainville: "Les veines du eorps naissent de " toutes les parties...., et il n'y a pas de doute qu'elles ne commu"niquent avec les artères par des vaisseaux de transition d'un » diamètre assez considérable. Je doute que ce réseau et même ces "vaisseaux aient dans toute leur étendue de véritables parois " vaseulaires, et je pourrais même affirmer qu'une grande partie eu » est entièrement dépourvue. »

Si Bojanus ne se fût point tenu à l'étude d'une seule espèce, il anrait bien vite acquis la conviction que les branchies ne sont pas des matrices, ear un grand nombre d'Acéphales ne portent plus leurs oufs pendant l'incubation; et eependant les prétendues matrices, qui, dans ces cas, seraient devenues inutiles, n’en existent pas moins.

J'ai rappelé ce fait pour montrer encore une fois combien il est nécessairc de multiplier les observations sur des espèces différentes et nombreuses, afin d'arriver à des données exactes.

De Blainville, qui s'occupa de faire connaître en France le travail dı célèbre professeur de Wilna, voulut aussi émettre son opinion qui n'avait rien d'arrêté, et qui, sous la forme d'hypothèse, attribuait suceessivement differents rôles à l'organe. Il se demanda si la glande ne serait point une annexe de l'organe de la respiration destiné à retenir de l'eau pour le cas où l'animal se trouverait placé hors de ce milieu; si elle ne représenterait pas un organe sécréteur appartenant à la dépuration du sang ou une sorte de rein; si elle ne serait pas une sorte de rate, de ganglion vasculaire, destiné a faire subir une modification au sang, avant d'arriver à l'organe de la respiration; ou bien enfin si elle n'appartiendrait pas à la génération, et si elle ne serait pas chargée de sécréter l'enveloppe des reufs.

Dans ces opinions, dans ees hypothèses, que, du reste, le savant professeur ne démontre pas, on trouve un peu tout, et par conséquent rien de préeis.

Je citerai l'opinion de Richard Owen : Modern analysis has " detected a large proportion of uric acid in the peritoneal con"partinent enclosing this veinous plexus, and has thus deternimed 
" it to be the renal organ. " Ainsi pour M. Richard Owen, celte glande est un rein, parce que les analyses modernes y ont décelé une large proportion d'acide urique (1).

Garner, dont j'ai souvent cité le travail vraiment remarquable sur les Mollusques, considère le corps de Bojanus comme un organe d'excrétion, qui doit rejeter certains produits hors de l'économie. Il observe une chose qui, à mon sens, a passé trop inaperçue , à savoir qu'à certaines périodes, le corps produit une plus grande quantité de matière calcaire. Ce fait est démontré par les sillons qui se voient sur la coquille, et qui correspondent, comme je l'ai observé même pour des embryons microscopiques, à des moments de repos. L'animal peut avoir besoin de se débarrasser, à certaines époques, d'un surcroît de matière calcaire, et l'organe glandulaire dont il est iei question rejette celte matière au dehors. C'est du reste l'opinion tout hypothétique de de Blainville, qui considère l'organe comme un rein dépurateur. Garner fait remar(puer la différence toutefois qui existerait dans celte espèce nouvelle d'organe dépurateur, qui aurait une circulation analogue à celle que l'on observe dans le foie des animaux supérieurs. Il avail, je l'ai déjà dil, connaissanee de la communication avec le périearde. Voici comment il l'indique pour l'Unio seulement : « In " the Unio an orifice elose to that of the oviduet, leads into a large " cavity of the mantle under pericardium, into which the secreting „organ opens by an internal orifice; " et il ajoute même que Bojanus u'aurait point soutenu son opinion s'il avait connu cette eommunication.

On trouve quelques erreurs touchant les rapports des orifices de la génération et du sac. Ainsi, d'après Garner, l'oviducte est dislinct du sae dans les Lithorlomes, les Moules; tandis que dans la Buearde, la Mactre, la Pliolade et la Mlye, les reufs sont rejetés daus le sae (2).

(1) Rich. Owen, Lectures on comparative analomy, vol. I, p. 284. Mais il ne cite pas les analyses, il n'y a vraiment pas assez de détails pour que cette plirase soit une véritable démonstration.

(2) Lor cit,, "Whilst in Tellina, Cardium, Mactra, I'holas, Mya and nost others the ova are discharged into the secretory organs, ") p. 29 i 
Il y a li une erreur, pour quelques espèces an moins La Bucarde, les Mactres, sont les animaux chez lesquels, au contraire, les deux orifices sont les plus éloignés, les plus séparés et les plus distinets (1).

Les concrétions nombreuses que l'on trouve dans le salc de Bojanus et dans son tissu même avaient été connues de Poli (2), et avant lui de Swammerdam (3). Ces deux auteurs avaient pensé, d'après la nature même de ces concrétions, que l'organe dont il s'açit avait pour róle spécial de sécréter la coquille. Cette opinion est aujourd'hui sans valeur : personne ne songe à la soutenir.

Von Siebold a eu aussi son attention fixée sur ces concrétions, et dans son Anatomie comparée, il s'exprime ainsi à leur égard : “... Le tissu très lâchı.... se décompose en petits corpuscules " granuleux et résiculeux; la plupart de ces corpuscules contiennent "un noyau arrondi... Ces noyaux sont très solides, et doivent être " considérés comme des corpuscules excrétés. Ils sont quelquefois n tellement grands, ‘u'on les apercoit à l'oil nu sous la forme de » concrétion inorganique, qu'on peut d'autant mieux comparer à des » calculsrénaux qu’ils contiennent de l'acide urique. » Et il ajoute en note : “Étant parvenu à recueillir une quantité considérable de ces " calculs, j'en ai remis une partic à $\mathrm{II}$. de Babo, de cette ville, qui » s'est chargé d'en faire l'analyse qualitative. Le résultat a été que ces " calculs, dont la cassure est conchoïde, sont composés principale" ment de phosphate calcaire avec une trace de phosphate de magné"sie, et une faible quantité d'une matière organique se comportant » avec l'acide nitrique exactement comme l'acide urique. " La fin de cette note se termine ainsi : "L'opinion qui les regarde comme des „ reins a trouvé beaucoup plus de partisans Treviranus dans Tie»demann, Zeitsch.f. Physiol., I, p. 53, et Carus, Zool., 1834, „ II, p. 650), d'autant plus qu'on affirmait qu'ils contiennent de "l'acide urique (Garner, Trans. of Phil. Soc. loc., p. 92, et Owen, „Lectures on the comp. Anat., p. 284), ce dont je n'ai pas pu m'as"surer jusqu’ì présent. La composition chimique de ces concré-

(1) Voy. la planche ö, fig. 6

(2) Ouvrage de Poli sur les Mollusques des Deux-Siciles.

(3) Biblie nuturex 
" tions me confirme dans l'opinion que ces organes sont réellement "des reins (1)."

Ainsi voilà l'opinion de von Siebold nettement formulée. L'organe de Bojanus est un organe dépurateur, et spécialement un rein.

Il est impossible de ne pas relever, après bien d'autres, du reste, l'erreur de Neuwyller (2) sur les prétendues fonetions génitales mâles de cet organe. Les spermatozoïdes qu’il croit avoir aperçus ne sont évidemment que des cellules chargées de cils, si longs quelquefois, comme on l'a vu.

II. Deshayes (3) considère l'organe comme jouant un rôle de dépuration, mais ne dit pas spécialement qu'il soit un rein.

Enfin M. Milne Edwards (4) avat depuis longtemps, dans 11 travail sur quelques animaux inférieurs de la mer, indiqué, à propos du sexe du Pecten, que ces glandes pourraient bien concourir a quelque titre à l'accomplissement des fonctions de la reproduction. Dans des conversations particulières, .1. Edwards, tout en donnant moins de valeur à cette idée première, m’a paru pencher aujourd'hui pour celle qui verrait dans l'organe de Bojanus un rein.

Telles sont les opinions que l'on trouve dans les ouvrages.

On ne peut tout d'abord s'empêcher de faire une réflexion en voyant quelles raisons déterminent l'opinion des auteurs. La plus vraisemblable, la plus accréditée aussi, est que le sac de Bojanus joue le ròle de rein. Or on se base sur la présence de l'acide urique, comme s’il était par avance démontré que la sécrétion urinaire était toujours caractérisée dans la série animale par la présence de cet acide. On se demande si la présence dans une partie de l'organisme de quelques concrétions se comportant comme de l'acide urique est bien une preuve que cette partic est un rein. Nous savons si peu sur les fonctions des animaux inférieurs, que véritablement

(1) Anatomie comparée, t. II, $2^{\mathrm{e}}$ partie, p. 280 , et note 5.

(2) Neuwyl., Neue Denkschr., VI, p. 25.

(3) Voy. Deshayes, Exploration scientifque de l'Algérie, Mollusques. Articles, Organes dépurateurs, des diverses Monographies.

(4) Voy. Milne Edwards, Observations sur la structure et les fonctions de quelques Zosphytes, Mollusques et Crustacés des cótes de Frunce (Annales des sciences naturelles, $2^{\mathrm{e}}$ série, 1. XVIII, p. 321 ). 
c'est aller un peu vite en se prononeant eatégoriquement sans autre preuve.

On appelie foie dans les animaux supérieurs une glande brunâtre qui sépare du sang un produit jaune verdâtre, la bile, et qui, particularité importante, se trouve sur le passage du sang revenant de l'intestin, e'est-à-dire sur le trajet de la plus grande partie des matières alimentaires absorbées après la digestion.

Quelle influence a sur ces matières alimentaires, désormais entrainées dans le torrent de la circulation, la glande hépatique? Nous n'en savons encore vraiment pas grand'chose. Si les faits avancés par M. Bernard se vérifient complétement, s'ils sont acquis à la science, nous commeneerons à entrevoir l'une de ces actions, et le rôle mystérieux du foie se dévoilera ì nous. Nous verrons les matières amylacées transformées par lui cu sucre ; mais resterait la bile, pour laquelle on doit encore se demander si ellc est un liquide nécessaire à la digestion, si au contraire elle n'est qu'une sorte de matière excrémentitielle, résultat d'une première dépuration opérée sur cette substance alimentaire qui fait son premier pas dans l'économie.

Quoi qu'il en soit, il ne peut être douteux pour personne que le foic n'ait un rôle importaut, et que ce rôle consiste à agir sur le sang chargé des principes alibiles autres que ceux qui sont versés dans le torrent circulatoire par le canal thoracique ou l'appareil chylifère.

Or, qu'on le remarque, le foic est justement placé sur le chemin du sang qui va au poumon, et à priori, ne scrait-on pas tenté de croire qu'un organe placé dans les mêmes conditions serait appelé à remplir les mêmes fonctions? Il faut bien le reconnaitre, ces considérations font naître dans l'esprit un doute sérieux ì l'endroit des fonctions du corps de Bojanus, qui se trouve précisément dans les conditions que nous venons d'indiquer. Il est placé sur le trajet du sang qui revient des organes de la digestion, et qui certainement est chargé des produits alimentaires. C'est done, si e'est un rein, un rein bien différent de celui des animaux supérieurs, car il est dans des conditions tout autres.

Et après ces réflexions, quand on rapproche d'elles les preuves vraiment bien légères que l'on donne de la nature de la fonction, 
on se prend à douter, surtout quand on songe an peu de notions ypue l'on possède sur les fonctions de ces animaux; car l'acide urique ne se présente pas toujours dans l'urine, et quand il se rencontre dans une partie du corps, nous n'en concluons pas forcément que cette partie est un rein. Ce qu'il serait utile de chereher, dans la liqueur excrétée, c'est l'urée, car l'urée est l'élément caractéristique de l'urine; mais encore de l'urine des animaux supérieurs. Or nous n'avons aucune donnée sur la sécrétion urinaire dans les animaux inférieurs.

Nous ne savons pas comment s'effectue la nutrition, par quelle voie et sous quelle forme sont rejetés les aliments ayant servi à l'accomplissement de la vie. Nous ignorons complétement si, de même que dans les animaux supérieurs, les matières azotées sont rejetées au dehors sous forme d'urée. On le voit, la question prend des proportions plus grandes qu'on ne le supposerait au premier abord. Envisagée sous ce point de vue, elle devient plus difficile à résoudre, et les preuves tirées de la présence d'un petit caleul d'acide urique, tout en ayant leur valeur réelle, n'en restentelles pas moins insuffisantes.

Quoi qu'il en soit de ces considérations, qui, du reste, montrent, je pense, tout le vague qui existe sur la question, voici les observations qu'il m’a été donné de faire :

Quatre espèces ont surtout présenté les faits les plus caractéristiques : ce sont la Nacre, la Lucine, la Lutraire et la Mactre.

En étudiant à Mahon les organes de la reproduction de la Nacre, je fusfrappé de la résistance des saes de Bojanus; en les ouvrant, je trouvais dans leur fond une poussière noirâtre, et dans leurs tissus des concrétions. Cette particularité n'avait rien d'exceptionnel, et dans tous les individus que j'observais à Mahon, dans ceux que je dus plus tard à l'obligeance de M. Valeneiennes et de M. Rousseau, je retrouvai constamment les mêmes concrétions.

L'examen microseopique de ces papilles qui hérissent la face interne des sacs, et qui constituent, comme on l'à vu, le tissu même de l'organe, montre (1) daus les cavilés de chacune d'elles une con-

(1) Voyez pl. 5, fig. 9 
crétion sphérique pertiforme, parfaitement arrondie, transparente, l'une teinte brunitre, terre de Sienne, offrant des lignes concentriques, 'pui indiquent, sans aucun doute, le dépôt de couches suceessives; on a sous les yeux une véritable petite perle. On se rappelle (que la cavité de la papille est oceupée par un canal sanguiu; et l'on se demande si e'est dans le sang que s'est formée cette conerétion perliforme? Je reviendrai plus loin sur cette formation et sur son origine.

On rencontre fréquemment, dans toutes les parties du corps des Naeres, des perles souvent allongées, pyriformes, ressemblant un peu à des larmes bataviques, ru’il serait bien intéressant d'étudier. Une analyse comparative de celles-ci, et des concrétions perliformes du corps de Bojanus, auraient, on le comprend, le plus grand intérêt. Malheureusement je n’ai point de données, et je ne puis dire si dans l'une et l'autre on rencontre les mêmes éléments; mais e'est li une laeune qui ne pourra et ue devra plus exister dans un nouveau travail.

L'organe de Bojanus de la Lucine [de celle de l'étang de Thau près de Cette, ef de eelle des environs des Hébiens près de SaintJacut-la-Nler en Bretagne (Côtes-du-Nord)] est vivement coloré en brun. On se rappelle que cette coloration est due au noyau des cellules du tissu. Ces noyaux, moins développés et très vagues dans les cellules les plus jeunes (1) et les moins grandes, deviennent de plus en plus nets, ct la matière colorante semble se coneréter et augmenter de plus en plus, à mesure que les cellules sont plus anciennes (2). Sur um même individu, il est rare que l'on ne reneontre pas tous les passages, depuis un simple noyau jusqu'i un petit eorpuseule sphérique composé de couches concentriques, disposées autour d'un centre plus clair, qui rappelle eneore par son apparenee ce noyau. On peut done admettre iei dans la Lucine qu'autour de ce noyau, comme centre d'attraction, est venue se déposer la substance calculeuse (3). Ces petites perles sont enfermées dans la cellule où elles se sont produites.

(1) Voy.pl. 4, fig. 11.

(2) Id., fig. $12(a b c)$.

(3) Id., fig 12 (c). 
Dans les Jambonneaux, on trouve les perles dans la cavité mème du vaisseau sanguin (1) occupant le centre de la papille. Comment s'expliquer cette position? Évidemment il y a eu rupture de la cellule dans laquelle s'était formée la conerétion, et chute de celle-ci dans la cavité interne. Si la rupture eût eu licu en dehors, la perle serait tombée dans le sac même, ce qui rend compte de la présence de cette poussière noire que l'on trouve dans la poche de l'organe.

Dans ces deux exemples, les dépôts sont irrégulierset amorphes ; dans ceux qui suivent, au contraire, il y a en cristallisation de la matière inorganique, et ici encore e'est autour du noyau que sont venus se grouper les petits cristaux.

Le corps glandulaire est, dans la Lutraire, d'un brun foncé ; si on le déchire sous l'ean, on voit s'en détacher une poussière brune qui se précipite au fond du liquide. Si on l'observe au microscope, on voit qu'il est formé de petites élévations peu transparentes, paraissant bourrées de petites aiguilles ou corpuscules allongés et dirigés dans tous les sens. Sur une portion peu considérable de la glande, à un grossissement suffisant, ces particules se font tout de suite reconnaître pour des cristaux (2), qui ressemblent singulièrement à l'acide urique que l'on rencontre dans bien des eas chez les animaux supérieurs. Mais, chose remarquable, e'est encore ce noyau (3), à bords irréguliers, parfaitement recomaissable, qui sert de centre aux cristaux. Ceux-ci sont groupés le plus souvent en croix; cependant il y a bien des variations de forme (4): tantôt le noyau est au centre d'une lamelle ellipsoïdale qui rappelle les lames rhomboïdales dont les angles sont arrondis, comme cela s'observe même dans les eristallisations de l'urine de l'homme; tantôt deux de ces plaques sont posées de champ l'une sur l'autre, et se coupent à des angles très variables, mais toujours le noyau est reconnaissableà l'intersection; tantôt enfin de petiles baguettes, plutôt aciculaires que véritablement lamellaires, rayounent eneore

(1) Pl. 4, fig. 5, fig. 9.

(2) Voy. t. IV, pl. 4, fig. 1.

(3) Voy. I. IV, fig. $1\left(n^{\prime}\right)$.

(4) Voyez les différentes formes de cristaux dessinées figure 1 de la planche 4 . 
du même point central, et l'on a, à n'en pas douter, le commencement d'un groupe analogue à ceux que l'on trouve dans l'urine de l'homme. Tous ces corpuscules sont enfermés dans des cellules, de telle sorte que, entre l'exemple actuel et ceux étudiés précédemment, il n'y a pour toute différence que la disposition amorphe dans un cas, la forme cristalline dans l'autre; et l'on peut ici dans cette différence apprendre à connaîtrel'origine même de ces petites perles.

Mais la Mactre présente dans le corps de Bojanus des groupes de cristaux qui rappellent véritablement, à s'y méprendre, les cristaux d'acide urique. La forme, la teinte, tout est parfaitement semblable (1). Sans connaitre les formes indiquées précédemment pour la Lutraire, il est difficile de comprendre leur origine et leur point de dejpart ; mais dans ce dernier exemple, on voit déjì le commencement du groupement des baguettes aciculaires. Ces baguettes, du reste, semblent rayonner d'un centre qui est. le noyau primitif de l'une des cellules. Ces groupes de cristaux paraissent noirâtres, quand on les observe à la lumière réfléchie; au contraire, ils sont rougeâtres, éclairés par la lumière transmise. Je ne les ai jamais rencontrés enfermés dans une cellude; cela tient sans doute à ce qu'ils avaient acquis déjà trop de développement, et qüils avaient rompu les parois. La cristallisation ou la production de matière inorganique, quoique placée en dehors de la cellule, n'en a pas moins continué et augmenté leur volume.

Depuis que j'ai fait cette observation, le même faits'est présenté de nouveau; j'en parlerai dans un travail que je me propose de publier bientôt sur l'anatomie et le développement de la Bullée (Bullca aperta). J'ai, en effet, rencontré dans le rein de cet animal des cristaux groupés tout à fait comme dans la Nactre des côtes de Bretagne.

Ces cristaux de la Lutraire ont été vus par M. Deshayes, qui les compare à des cubes à angles arrondis, à des navicules et des tourniquets compresseurs; il observe qu'ils sont solubles dans l'acide nitrique, mais il n'en indique pas la nature. Du reste, tous les

(1) Voy. t. IV, pl. 4, fig. h. 
auteurs ont été frappés de la présence de ces concétions solides inorganiques ; Poli avait même basé sur elle sa théorie de la sécrétion de la coquille.

Il était bien intéressant, on le comprend, de connaître la composition de ces conerétions ; aussi en avais-je recueilli dans le but de les faire analyser. Toutefois, malgré les résultats que mon excellent ami et collaborateur pour d'autres travaux, M. A. Riche, a obtenus, je croisqu’il faudrait procéder à de nouvelles études. Pour moi, en effet, les analyses chimiques, quand elles s'appliquent à la physiologie, doivent avoir un but défini ; on doit chercher dans telle ou telle direction, et ce n'est pas seulement d'une analyse isolée que l'on peut conclure quelque chose de positif. Des analyses isolées fournissent des renseignements sans doute; mais pour arriver à des faits caractéristiques, il faut des recherches comparatives. $\Lambda$ insi les Jambonneaux présentent des concrétions dans le sac de Bojanus, dans les tissus du manteau, ete., etc. N'est-il pas évident qu'il faudrait faire l'analyse comparative de ćes produits, et n'est-il pas hors de doute que leur composition devrait être opposée à celle de la coquille elle-même? On le voit dans l'étude qui nous occupe, ces recherches prennent des proportions considérables, surtout quand on remarque que ce n'est pas sur une espèce isolée, mais évidemment sur un grand nombre que le travail doit être entrepris.

Je ne présente done qu'avee réserve les quelques résultats que je dois à l'obligeance de mon labile ami. Voici le passage même de la lettre où il me rend compte des opérations qu'il a lait subir aux corps que je lui avais remis.

"Les masses brunâtres que vous m'avez dit appartenir ì la Lu„ traire solénoïde ont été desséchées à 50 ou 60 degrés, puis „ mises en digestion à chaud avec une solution de potasse ; la solu„ tion, décomposée par l'acide chlorhydrique, a donné un précipité „ blane d'acide urique insoluble dans l'alcool et dans l'éther. Séché „ sur un filtre séparé du papier, ce précipité, traité par l'acide azo» tique, avee la chaleur et la vapeur d'ammoniaque, a donné la " coloration rouge caractéristique de l'acide urique. "Déjà, on se le rappelle, la simple observation microseopique m'avait conduit à admettre la présence de cet acide. L'analyse iei est démonstra- 
live; clle a quelque chose de plus positif que celle rapportée par von Siebold et faite par 11 . de Babo.

Dans la série des matières que j’avais données à M. Riche, se trouvait, mais en petite quantité, la glande de la Vactre, où l'on observait très nettement les cristaux paraissant d'acide urique, et dontj'ai parlé. Traitée de la même manière, un précipité très faible s'est encore produit; il était insoluble dans l'aleool et l'éther, et coloré légèrement en rouge par la réaction de l'acide azotique et de l'ammoniaque; mais la quantité de matière était très faible, et il y avait, bien que la réaction se présentât, moins de certitude que précédemment.

Mais, chose curieuse dans les eonerétions de la Pinne marine, conerétions perliformes et non cristallines, l'acide urique ne s'est point montré, si du moins on juge de sa présence par les réactions précédentes. Cependant le même procédé a été employé à plusieurs reprises, ear la quantité de matière était plus considérable.

M. Riche a cru y trouver de l'urée. "Il m'a paru y en avoir un " peu. J'ai essayé le dosage au moyen du procédé de M. Millon; je "n’ai eu que des traces d'acide carbonique, dégagé par l'action de „la substance sur l'azotite de mercure dissous dans l'acide azo" tique. L'eau de chaux était troublée cependant, et en recueillant » le gaz dans un tube à potasse, j'ai eu une augmentation bien légère " de poids due à l'acide carbonique produit. "

Malheureusement les analyses n'ont eu pour but que la recherche de l'acide urique et de l'urée. Elles auraient dù aussi faire connaitre la nature même des calculs qui ne présentaient pas d'acide urique, la substance manquait pour cela ; mais je ne puis admettre néanmoins que ces derniers résultats infirment les premiers.

En effet, dans la vessie de l'homme se forment des calculs d'acide urique, mais tous ne sont pas d'acide urique. Il en est de phosphate ammoniaco-magnésien (ce sont les plus fréquents) où l'vn chercherait en vain l'acide urique, et ee n'est pourtant pas ì dire qu'ils ne soient earactéristiques de la sécrétion urinaire. Y aurait-il ici quelque chose qui rappelle ce que l'on voit dans l'homme. L'acide urique est plus fréquemment cristallisé, tandis que le phosphate ammoniaco-magnésien est presque toujours amorjhe, dépose 
par couches concentriques. Je regrefle beaucoup de n'avoir point la composition du sel qui forme les concrétions perliformes du corps de Bojanus du Jambonneau.

C'est surtout l'urée qu'il serait important de chercher et de trouver; mais les difficultés sont extrêmes dans ce travail. En effet, l'organe est peu volumineux dans les espèces que nous pouvons facilement étudier.

Il faut done le prendre sur un grand nombre d'individus. Or le sang qui est dans son intérieur est abondant, et il s'écoule en grande abondance par les blessures que l'on fait à l'animal; on a done les glandes de Bojanus baignées dans une masse considérable de ce liquide. Or on ne sait rien sur la composition chimique du sang des Acéphales ou des Mollusques; on se voit toujours en face de bien des questions quand on veut aborder les études de chimie physiologique des animaux invertébrés.

Des faits qui précèdent, il semble découler, avee les observations des auteurs que j’ai cités précédemment, que la glande de Bojanus est un rein.

Cependant, je l'avoue, il m'est difficile de croire que si, en effet, cet organe est chargé de séparer du sang ce que nous nommons dans les animaux supérieurs l'urine, il soit exclusivement destiné à remplir ce rôle. En effet, j'ai bien des fois été frappé, et cela avant de connaitre l'opinion de M. Edwards (1), par la coïneidence évidente qui existait entre le développement des sacs de Bojanus et celui des glandes génitales. A Mahon, aux Martigues, à Cette, à la Rochelle, à Saint-Malo, à Courseulles, j’ai maintes fois observé que les animaux, au moment de la ponte, avaient leur corps de Bojanus vivement coloré et fort turgide; que ce développement, annonçant une plus grande activité de la sécrétion, cessait ou diminuait beaucoup, au contraire, sur les individus chez qui la périorle d'excitation génitale était passée.

Parmi les nombreux faits de ce genre quej'ai observés, je citerai l'un d'eux, que je trouve dans mes notes de $\mathbf{1 8 5 4}$ sur mon voyage en Bretagne.

(1) Loc. cil 
Aux grandes marées d'atout, j'ilvais tromvé sur les plages des Ilébiens beaneoup de Pandores rostrées dont les organesde la génération étaient gorgés par les produits de la sécrétion, et je me demandais si je ne pourrais en étudier la ponte et le développement. J'avais remarqué que les corps de Bojanus étaient vivement colorés, les corpuscules nucléolaires étaient fort nombreux et très gros. Comme ces Pandores habitaient un fond sablonneux assez profond, je lus empêché dans ces recherches par la morte-eau ou petite marée de la première quinzaine de septembre. A la grande marée suivante les Pandores avaient pondu, et je trouvai tous les organes génitaux vides. Chose remarquable, les glandes de Bojanus avaien! perdu la vivacité de leur coloration, et il ne pouvait être douteux que la cessation du travail d'une glande ne cö̈neidât avec celui de l'autre. Dans beaucoup d'autres circonstances, cela m'a paru si évident que, dans les notes de mon voyage aux Baléares, je trouve le corps de Bojanus souvent désigné par le nom d'annexe de la génération. On se rappelle d'ailleurs le rapport presipue constant qui existe entre les orifices des denx glandes.

C'est en rapprochant ces faits de ceux que l'anatonie nous a montrés à propos de la circulation, que je n’ai pu, malgré les an:lyses, arriver à prendre une détermination absolue, avant d'avoir préalablement fait de nouvelles recherches.

Je suis loin cependant de nier que les saes de Bojanus soient des organes dépurateurs analogues aux reins; mais je croirais volontiers qu'au lieu d'un seul rôle ils peuvent en jouer deux. Ainsi nous voyons dans la série animale, ì mesure que l'organisme se simplifie de plus en plus, la division du travail être de moins en moins grande, et un même organe remplir plusieurs fonctions. N'y aurait-il pas ici quelque chose de semblable, et la glande rénale de quelques auteurs ne pourrait-elle aussi devenir glande anmexe de la génération à un moment donné, tout en conservant sou rôle d'organe dépurateur?

En résumé, on le voit par les détails que je viens de dommer, la nécessité de recherches phỵsiologiques sur l'ensemble des fonctions des animaux inférieurs ne doit faire aucun doute; rt e'est paree que les notions que pussìde la science ì cet égard me 
paraissent trop insuffisantes, que j'ai apporté une grande réserve dans les conclusions de mon travail.

Je citerai en terminant un dernier fait. Sur une Mactre qui présentait les cristaux d'acide urique dont j'ai parlé, je trouvai aussi dans l'ovaire une foule de pelits corps brunâtres, qui n'étaient rien autre que des calculs; et en étudiant minutieusement le tissu, je rencontrai dans un ouf $(\mathbf{1})$, entre la coque et le vitellus, un calcul, une masse de substance pierreuse. Il est difficile de trouver la matière calculeuse plus avant dans l'organisme. Or ne se pourraitil pas faire que les Mollusques produisant des perles, quels que soient le nom ou la valeur des produits, ne fussent autre chose que des animaux placés dans une même condition que l'hornme goutteux, ayant une diathèse calculeuse, une disposition à laisser précipiter dans tous les points de l'économie cette matière calcaire qui, normalement, se dépose par couches successives et forme la coquille? N'est-il pas probable que, de même que l'acide urique et le phosphate ammoniaco-magnésien se déposent, chez l'homme et les animaux supérieurs, dans la vessie ou autour des articulations pour former les calculs vésicaux, ou les concrétions tophacées des calculeux et des goutteux, de même ici, quand les matériaux se développent anormalement et outre mesure, ils peuvent se déposer partout, et donner naissance aux calculs que nous avous trouvés dans le corps de Bojanus, ou bien aux perles proprement dites? En uII mot, le Mollusque produisant des perles n'est-il pas un être atteint d'une diathèse calculeuse?

Je me suis abstenu de donner un nom particulier à la glande, rela se comprend sans peine. La résignation que j’ai employée, corps de Bojanus, ne préjuge nullement le rôle. Que d'exemples de dénominations semblables je pourrais citer dans l'anatomie de l'homme! Les corpuscules de Malpighi désignent des choses que l'on ne peut confondre avec d'autres, et cependant le nom ne lait en rien pressentir la fonction. Il était prudent d'ailleurs de se garder d'imposer un nom basé sur les fonctions, puisque je n'avais rien de positif, de fixe à leur égard; il me suffirait enfin de rappeler

(1) Voy. t. IV, pl. 4, fig. 5 (c). 
'que les noms de poumon, testicule, rein, organe dépurateur, appendice veineux, donnés successivement à l'organe, suivant les opinions différentes des auteurs, ont dù être tour à tour abandonnés, ce qui n'eùt pas eu lieı, si à la place d'un nom significatif on eût employé un norn sans valeur physiologique.

\section{EXPLICATION DES FIGURES.}

Anatomie de l'organe de Bojanus.

PLANCHE 4.

Fig. 1. Cristaux de formes diverses, libres ou enfermés dans des cellules, trouvés dans le tissu du corps de Bojanus de la Lutraria solenoïdes. $(n)\left(n^{\prime}\right)$ noyau, (f) cellule.

Fig. 2. Éléments microscopiques isolés de la même glande dans la Lima squamosa. On croirait à l'existence d'une seconde cellule incluse.

Fig. 3. Éléments microscopiques isolés de la Corbula striata. Ces éléments sont de deux grandeurs; les plus petits portent les cils vibratiles.

Fig. 4. Un groupe de baguettes aciculaires de la Mactra stultorum. La ressemblance avec l'acide urique de l'homme est extrême.

Fig. 5. Un œuf de la même, ayant entre sa coque et son vitellus une concrétion pierreuse (c).

Fig. 6. Corps du Spondylus Gcederopus dépouillé du manteau et des branchies , pour montrer le corps de Bojanus $(r)(r)$ ouvert; $(o v)$ orifice de l'oviducte; $(p e)$ orifice du sac; $(m p)$ muscle des valves.

Fig. 7. Portion de tissu de la glande du même (b); cils vibratiles (c); corpuscules moins développés que ceux placés en $(a)$.

Fig. 8. Corpuscules du même très développés, et qui se trouveraient après ceux marqués $(a)$, fig. 7 .

Fig. 9. Une portion grossie de la substance du même, pour montrer l'apparence veloutée de la face interne.

Fig. 10. Tissus du corps de Bojanus de la Chama griphoides.

Fig. 11. Tissus du corps de Bojanus de la Lucina lactea peu développé; la matière colorante est encore disséminée, diffuse.

Fig. 12. Id. : en $(a)(b)(c)$ on voit le noyau se concréter de plus en plus, et en (c) il est devenu le centre d'une véritable petite perle. On voit dans ces cellules un second cercle, qui semblerait, comme dans la figure $\mathbf{2}$, indiquer une seconde cellule incluse.

PLANCHE 5.

Fig. 1. Portion (b) du tissu du corps de Bojanus de la Petricola ruperella, montrant l'épaisseur des parois des lobules et leur disposition; (a) éléments isolés. 
Fig. 2. Coupe un peu théorique de l'organe $(v)$ dans l'Unio pictorum; $(p)$ péri carde ; $(c)$ cœur ; $(o v)$ oritice génital ; $(p e)$ orifice externe de la poche périphérique $(c p) ;(p i)$ orifice péricardique ou interne de la poche centrale $(c c)$; ( $m p)$ muscle postérieur des valves.

Fig. 3. Papille de l'Arca Noe fendue, pour montrer l'orifice génital dans la paroi.

Fig. 4. Deux lobules de l'organe dans le Cardium rusticum, pour montrer les dépressions glanduleuses qui semblent renverser la disposition habituelle.

Fig. 5. Éléments microscopiques du même.

Fig. 6. Corps du même vu en dessous, pour montrer $(p)$ le pied, $(b r)$ les branchies, $(g b)$ les ganglions branchiaux, (co) le connectif bucco-branchial, (ov) l'orifice de l'oviducte, (pe) l'orifice externe du sac de Bojanus.

Fig 7. Porlion de tissu de la Modiola lithophaga, pour montrer deux apparences $(a)(b)$, et le commencement d'une dépression conduisant à la forme renversée, figure 4 .

Fig. 8. Ėléments isolés du nıême.

Fig. 9. Une portion du tissu de l'organe de la Pimna nobilis, montrant dans le centre de chaque lobule une perle.

Fig. 10. Animal de la Modiola lithophaga, vu de profil, pour montrer $(r r)$ le corps de Bojanus, $(o v)$ la papille orifice de l'oviducte et du corps de Bojanus; $(m v)$ la masse viscérale, $(p)$ le pied, $(m p)$ le muscle postérieur des valves.

Fig. 11. Corps de Bojanus avec les parties voisines de la Petricola ruperella, pour nontrer ses rapports. Les mêmes lettres signifient les mêmes choses que précédemment.

Fig. 12. Id, de la Carlita sulcata.

Kig. 13. Corps de la Chama griphoïdes, pour montrer l'organe de Bojanus. Mèmes lettres désignant même chose que dans les figures précédenles.

liig. 14. Eléments microscopiques du Pecten glaber

Fig. 15. Extrémité d'un lobule de la Pandora rostrata, remarquable par la netteté des corpuscules solides et la longueur des cils vibratiles.

Fig. 16. Éléments du Pectunculus pilosus. L'une des cellules, plus grande, semble en contenir quatre.

\section{PLANCHE 6 .}

Fïr. 1. Corps du Pecten jacobuus vu de profil, et débarrassé du manteau el des branchies pour montrer $(f)$ le foie, $(j)$ les vaisseaux sanguins veineux nés dans cette glande, $(c)$ le cœur, $(b)$ la bouche, $(p)$ le pied, $(r)$ le rectum, (a) la masse abdominale, (pe) l'orifice externe de l'organe de Bojanus, $(y)$ le vaisseau unique qui résulte de la réunion des vaisseaux du foie, $(s)$ le sinus qui reçoit le sang du muscle postérieur des valves et autres parties et le verse dans les branchies.

lig. 2. La branchie (ur) droite est conservée, rejetée à gauche, pour laissel voil (h) les vaisseaux veineux qui, de l'organe de Bojanus, portent le sang au sinus branchial $(s b)$ qui se continne en une veine $(r b)$, sinus qui verse le 
sang du muscle et des parties postérieures du corps dans le sinus branchial $(s b)$.

Fig. 3. Péricarde ( $p r)$ du Cardium echinalum ouvert par le dos, le cœur enlevé, pour montrer les orifices internes ( $p i)$ de l'organe de Bojanus.

Fig. 4. Organe de Bojanus isolé et injecté de la Lutraria solenoïdes, vu par la face dorsale, avec les sinus $(l)(0)(n)(q)$ ouverts; $(m)$ est la série d'orifices des vaisseaux branchiaux; $(r)$ rectum; $(p i)$ orifice péricardique de la cavité profonde de l'organe; $(v b)$ veines branchiales.

Fig. 5. Organe de la même vu de profil $;(n, o, q, t, m)$ mêmes choses que dans la figure précédente; $(c)$ cœur.

Fig. 6. Id., l'organe ouvert, pour montrer les vaisseaux qui se distribuent dans les tissus; $(\mathrm{sm})$ sinus médian inférieur résultant de la réunion des veines du foie; $(n)(i)$ branche collatérale destinée à la partio désignée spécialement par Bojanus sous le nom de poumon; $(p)(l)$ vaisseau terminal du sinus; $(c)$ cœur ; (z) orifice de communication entre les deux poches externes de la cavité externe.

Fig. 7. La même figure que la précédente, mais vue de face et en dessous, pour montrer les mêmes parties; le sinus médian impair $(s m)$ ouvert et logeant les deux connectifs partant du ganglion $g$.

Fig. 8. Apparence des capillaires à la surface du corps de Bojanus dans le Pecten maximus. 





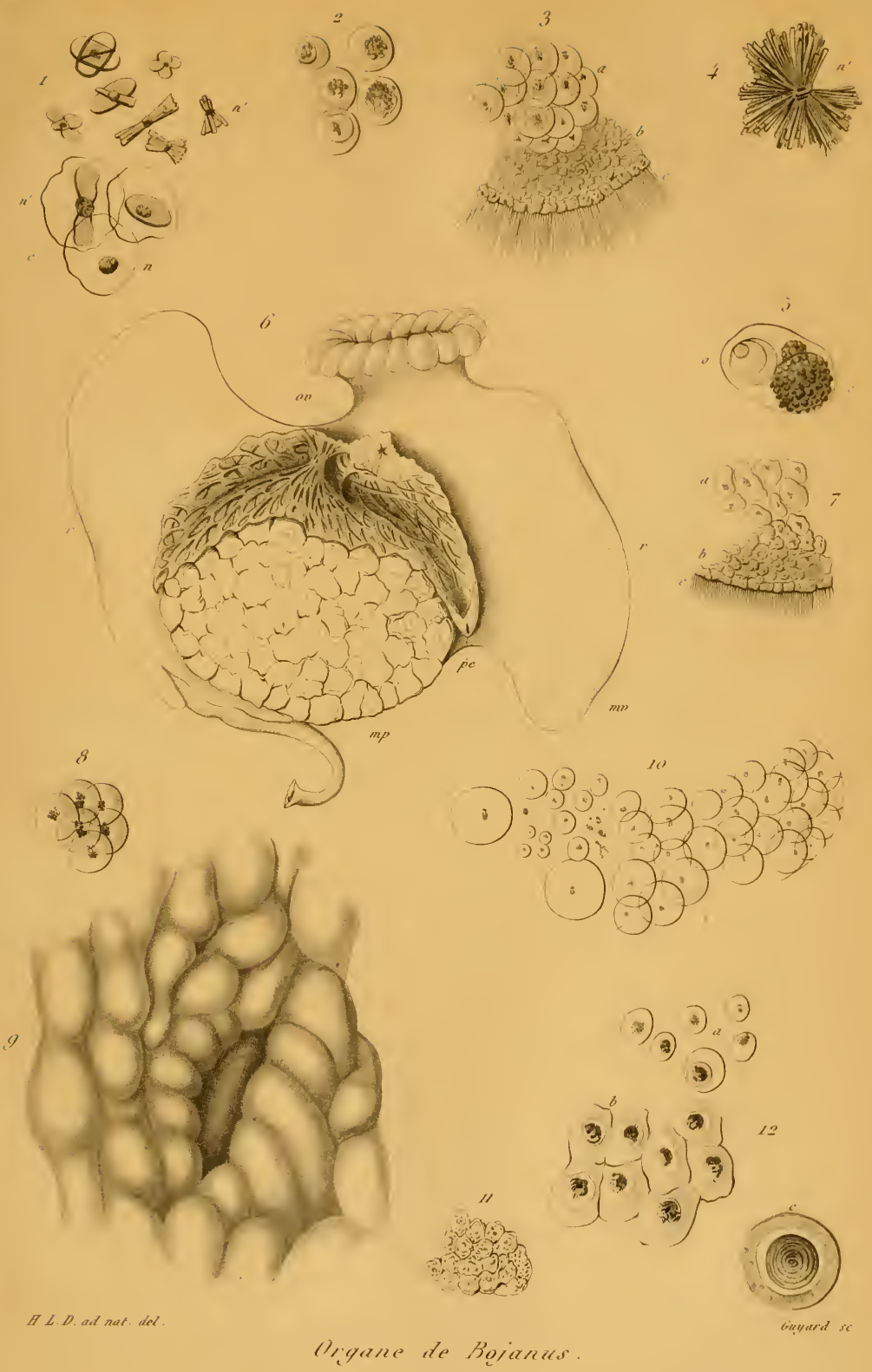




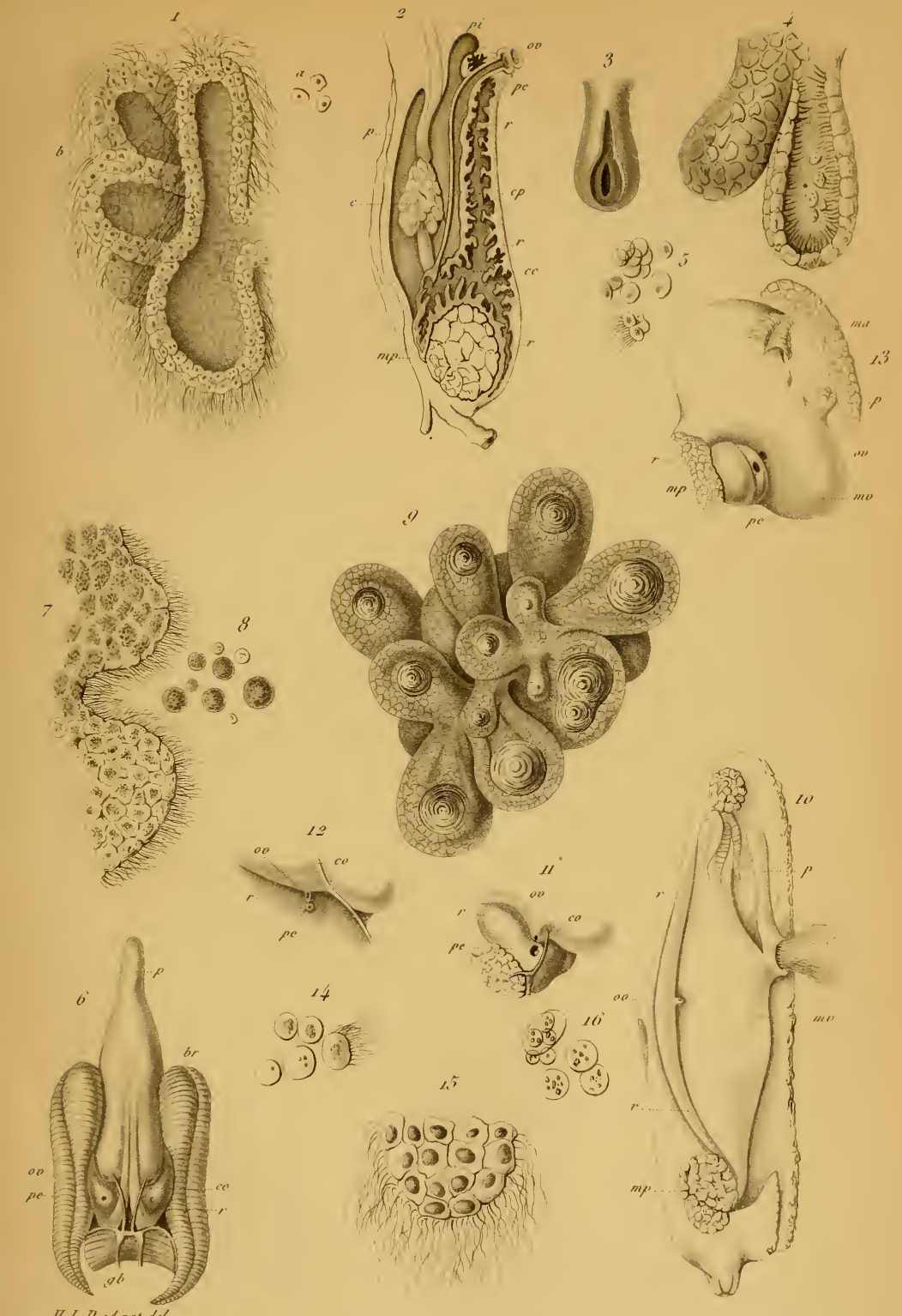

Orqune de Bojunus. 



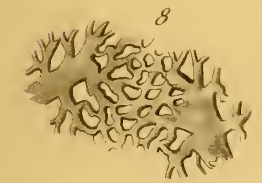

"LDD
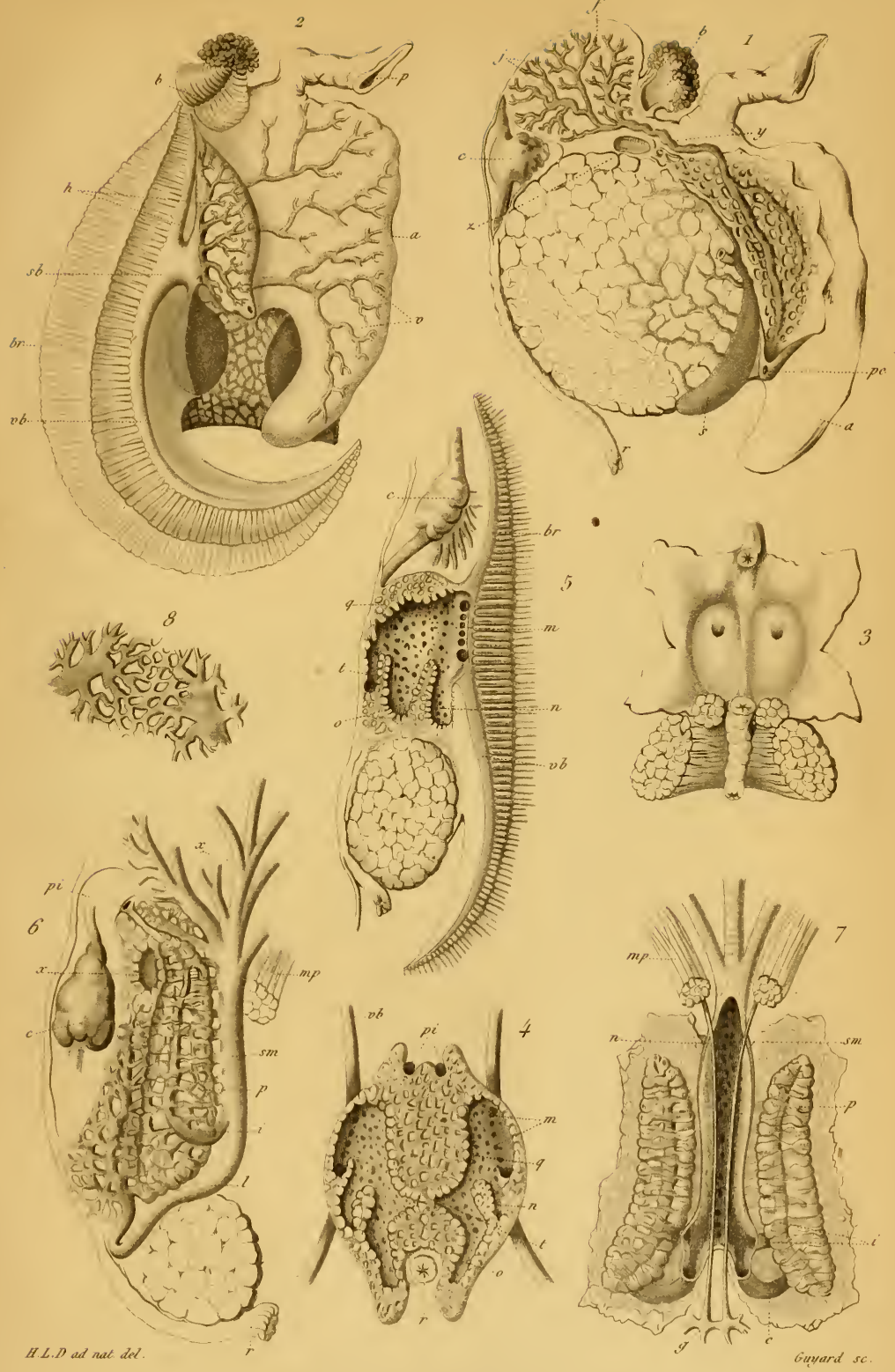

if 7ool. Tome + PLo

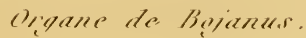





\section{MÉMOIR E}

SUR

\section{LE DÉVELOPPEMENT DES BRANCHIES}

DES MOLLUSOQUES ACÉPHALES LAMELLIBRANCHES.

I.

HISTORIQUE.

L'embryogénie des Mollusques, considérée dans l'ensemble de l'embranchement, est encore peu avancéc ; celle des $\Lambda$ céphales en particulier est surtout en retard. Quelques mémoires peu nombreux ont fait connaître des faits importants, mais aucun d'eux ne présente l'histoire entière d'un organe depuis l'origine jusqu'au développement complet.

Rien cependant n'est utile en zoologie comme les connaissances d'embryogénie; chaque jour on voit de plus en plus l'importance des recherches qui se rapportent ì cette branche de la physiologie. On peut même, sans être taxé d'exagération, affirmer que tout l'avenir de la science des animaux est maintenant dans la connaissance des transformations embryonnaires (1).

Ne voyons-nous pas, en effet, non-seulement des espèces, des genres, mais encore des ordres, des classes, disparaître des cadres

(1) Voyez Considérations sur quelques principes relatifs à la classifization naturelle des animaux, par M. Milne Edwards (Ann. des sc. nat., $3^{\mathrm{e}}$ série, 1844, t. I ${ }^{\mathrm{er}}$, p. 65 ). 
zoologiques depuis les laborieuses et savantes recherehes des Van Beneden, Siebold, Leukart, Küchenmeister, Phillippi, ete., etc. Il est permis de dire qu'il arrivera un moment où il ne sera plus possible d'assigner une position zoologique à un animal, sans connaître les formes qu'il a dû présenter depuis l'œuf qui l'a produit; alors seulement on aura une idée exaete des rapports zoologiques dans toute leur étendue.

Heureusement il faut le reconnaitre, la science semble entrer dans une voie nouvelle, et tout fait espérer qu'elle arrivera à des résultats bien autrement importants que ceux qu'ellẻ a déjà fournis par l'énumération de caractères qui ne sont que la somme d'un compte bien fait et patiemment fait de toutes les particularités observées sur les diverses parties du eorps d'un animal.

Le classificateur ne peut plus se contenter des formes que présente un senl individu à un moment donné de son existenee; il doit loaser ses divisious sur l'ensemble des formes correspondant aux différentes phases de la vie. C'est là ee qu'ont senti les zoologistes modernes, et ee qui explique l'ardeur avee laquelle ils se livrent ì l'éhude si diffieile du développement des animaux.

C'est aussi ce qui m'a conduit à faire les recherches, objet de ce mémoire.

Mon intention était et est encore d'étudier l'embryogénie comparée aussi eomplétement que possible de l'embranehement des Mollusques. Déjà j'ai réuni des matériaux nombreux ; mais une position nouvelle m'a détourné, pent-être pour longtemps, de ees études ; il m'a paru intéressant cependant de faire connaître quelques points de mon travail. Ils sont entièrement nouveaux.

J'ai eu le désir de faire voir comment naissait ou apparaissait un organe; par quelles transformations sueeessives il passait pour arriver à son état parfait. J'ai voulu montrer anssi que l'étude de l'évolution génésique d'une partie explique facilement certaines formes ou dispositions dont rien ne peut faire appréeier la cause et l'origine chez l'arlulte.

Ce travail, déjì fait pour bien des organes chez les animaux qui se rapprochent le plus de l'homme, u'a pas éti entrepris pour les 
Mollusques. Lì, en effet, l'embryogénie, peu avancée, se borne à des généralités; elle s'en tient à peu près exclusivement à des dounées générales sur les premières phases de la vie. J'ai done cru devoir faire pour charjue appareil une étude spéciale, et aujourd'hui je veux m'oceuper de celui de la respiration.

Sans avoir une application zoologique aussi directe que sembleraient le faire croire les lignes qui précèdent, les faits rqui suivent montreront, je l'espère, comment se constitue l'appareil de la respiration.

Nos connaissances, qui sont déjà très bornées en ce qui touche l'embryogénie générale des Lamellibranches, le deviennent encore davantage quand il s'agit du développement d'un organe, et en particulier de celui de la respiration.

Les seuls travaux sérieux que nous trouvions sur cette partie de l'histoire des Mollusques sont ceux de MM. Carus, Löven et de Quatrefages. Ils nous font connaitre le développement de quelques Acéphales seulement pendant les premières périodes.

On trouve bien encore dans les Mémoires de la Société biologique de Paris un travail sur le développenent de l'Huittre; ce qui se rapporte dans cet opusenle aux organes de la respiration ne mérite guère d'être considéré comme étant sérieux.

Carus (1) avait déjà depuis longtemps fait connaitre quelques faits sur le développement des Naïades (Anodontes); il faut le dire, les résultats d'alors ne sont plus guère en rapport avee les progrès de la science d'aujourd'luui.

I. de Quatrefages avait aussi étudié l'embryogénie de l'Anodonte (2); mais son travail le plus moderne est celui qu'il a publié sur le Taret. N'ayant pu continuer ses observations si curieuses et si habilement conduites, le savant académicien n'a pas dû trouver' la signification de quelques parties, qui, sans aucm doute, sont les premiers rudiments des branchies. "Il s'est développé dans ce " point, dit-il (dans l'amas de globules placés dans ce voisinage de "la (harnière), deux organes très singulier's : ce sont deux ouver-

(1) Voyez Carus, dans les Actes des curieux de la nature, XVI, 1832.

(2) Voyez de Quatrefages, Sur la vie inlrabranchiale des jeunes Anodontes (Ann. des sc. nat., $3^{\mathbf{e}}$ série, t. IV et V). 
"tures placées à côté l'une de l'autre, et garnies d'un fort bourre» let cilié. Les cils de l'une en s'agitant produisent exactement „l'effet d'une roue dentée en mouvement; ceux de l'autre ouver" ture n'offrent dans leur aspect rien de particulier. J'ai répété s cette observation sur plusieurs larves, et toujours j’ai trouvé »cette différence (1). " On le voit, les organes de la respiration sont indiqués dans ce travail, sans que leur signification soit assignée : elle ne pouvait l'être, puisque ce n'est que plus tard, longtemps après probablement l'époque à laquelle M. de Quatrefages cessait ses observations, que se termine le développement.

M. Löven a publié dans les Mémoires del'A cadémie de Stockholm un travail fort étendu sur le développement des Acéphales lamellibranches. Ce travail a une grande importanee, et mériterait d'être connu ; il est fächeux que sa tradıction n'ait point paru dans un reeueil périodique français. On y trouve décrite l'origine des branchies, qui correspondent aux fentes en boutonnière ciliées, indiquées par II. de Quatrefages ; mais, après avoir constaté l'apparition de l'organe, le savant suédois ne cherche pas ì en suivre les progrès jusqu'à la constitution complète; il s'arrête, et ne pousse pas assez loin ses observations pour avoir une idée complète du développement; s'il hasarde quelques explieations, on voit que e'est d'une manière dubitative, et que les faits semblables à ceux qui font l'objet du travail que je publie lui ont manqué complétement.

Je citerai le passage entier où il est question de ce développement (2) :

“Les branchies sont ehez tous (les embryons des acéphales ") lamellibranches) plus ou moins développées, nous avous remar" qué chez les Montacuta (3) qu'un cordon délié offrant trois ren" flements sensibles sort de la paroi postérieure du manteau, et se

(1) De Quatrefages, Ann. des sc. nat., $3^{\text {e }}$ série, t. II, p. 217, Études embryologiques, Mémoire sur l'embryogénie du Taret.

(2) Voyez Mémoires de l'Académie de Stockholm, 1848. - Kongl. ventenskaps akademiens Handlingar fö ar 1848 , p. 3 \$9. - Titre du mémoire: Bidrag Lill Kä̈nnedomen om utvecklingen af mollusca acephala Lamellibranchiala, af Löven Hartill, tab. $\mathrm{X}-\mathrm{XV}$.

(3) Genre créé par l'auteur. 
"perd bientòt sur le foie (lig. 104, 105, 107 et 108, y); et je "présume que ce sont là les premiers rudiments des branchies. "Dans la figure 116, elles manquent entièrement; car il est diffi" cile de les retrouver dans ce corps oblong, transparent, presque " vésiculeux, qui parait derrière la bouche, et dont la nature m'est "restée inconnue jusqu'à présent. Mais dans la figure 113, $y$, on " voit sortir du manteau, en faisant des anses, un cordon allongé, "renflé par intervalles (peut-être creux), et qui est régulièrement " contourné en quatre areades, dont la dernière va se perdre con") fusément derrière l'nesophage. Dans la figure 111,y, on voit le " mème organe, mais il est ramassé en anses arrondies. Si nous "passons à la figure 107, $y$, nous voyons ces anses sortir du bord "du manteau, et gagner ou augmenter de volume. Peut-être les "lobes arrondis sortant des bords du manteau ne sont-ils qu’une "anse imparfaite; quant aux anses suivantes, elles sont distinctes, " mais disposées de telle sorte quie leur ouverture ne parait pas "complète. Les troisième et quatrième sont très évidentes; ces " dernières se perdent dans un pli au-dessous de la bouche. On les " voit de même dans les figures 112 et 115,y, et plus développées, "sortant dubord postérieur du manteau jusqu'aux environs de la " tête où elles se fixent. Dans cette partie, leur ouverture est mani"feste au centre; elle est garnie intérieurement de cils, serrés, "développés, ressemblant à un repli quand ils sont en repos, ou " déterminant des muages onduleux, comme des vagues, yuand ils "sunt en mouvement... On ne peut pas douter yue ces grands sorganes ne soient des branchies, quoique cependant je n'aie " jamais pu me convainere qu’il y en eût de chaque côté; car je. " n’ai jamais distingué qu'un seul jli, bien que j’aie cherché les cils " internes pour découvrir la série interne des branchies.

"On doit remarquer ici qu'il existe des Mollusques qui n'ont " yu’un seul feuillet de chayue eoté; celui-ci se sépare peut-ètre " en deur très longtemps après.

"Les anses ne sont pas libres en haul; une ligne très fine, un "pen ondulense, indique tine membrane tig. 112, y), qui parait " limiter le canal par lequel la braurhie se réunit arre le vaisseau - de lia cirrulations. 
"Nous avons, si je ne me trompe, vu la première formation des "branchies; nous en savons assez pour être sùr qu'elles se mon" trent sous la forme d'un cordon fin, renflé à certains intervalles; "que ces renflements se contournent plus tard en anses, qui "s'allongent de plus en plus, et sur lesquelles se développent les „ cils vibratiles régulièrement disposés, et d'une forme particulière.

„Ce sont ces anses, si ouvertes et si arrondies, qui s'allongent, " plus tard, en se développant, et restent grêles, étroitement unies „et serrées; ce qui fait qu'on peut à peine reconnaìtre leur ancienne » forme. C'est ainsi qu'elles produisent ces feuillets considérables » qui, fixés dans la partie antéricure et interne des lobes du man" teau, constituent une portion très grande de l'animal.

"Quand les organes de la respiration sont parvenus au déve» loppement que nous voyons ici, le cour ne tarde pas à se former " rapidement; mais je n'ai jamais été assez heurcux pour l'observer » chez aucun des Acéphales que j’ai examinés, et je désespère même "de le faire (1). "

Ainsi il n'est pas douteux que M. Löven n'ait vu l'origine des appareils respiratoires; mais aussi qu il n’a pas observé les transformations diverses qui conduisent aux diflérents feuillets; je pense même que la supposition qu'il fait pour expliquer leur production n'est pas entièrement conforme à ce que l'on va voir chez la Moule.

Ainsi la partie historique est très restreinte, et nous n'aurons pas à expliquer et à accorder entre elles les différentes opinions, puisqu'elles se rédıisent à celles de M. Löven, M. de Quatrefages n’ayant pas assigné de fonctions aux fentes ciliées qu'il avait vues.

Quant à celles du recueil de la Société de biologie, ellesn'ont de rapport avec rien de ce ıui a été vu par les auteurs; leur inexactitude me parait telle, que e'est à peine si elles doivent trouver place dans une eritique séricuse.

(1) Cette traduction du mémoire a été faite par M. Yung, employé du laboratoire d'entomologie du jardin des plantes de Paris. Je le prie de recevoir mes remercîments pour l'obligeance qui il a bien voulu mettre à me fournir le passage que je viens de citer. 
II.

BRANCHIE DE LA MOULE ADULTE.

Pour bien s'entendre dans la deseription de l'organe naissant de la respiration, il est nécessaire de voir quelle est la composition de la branchie d'une Moule adulte (Mytilus edulis des marchés de France), et de fixer la valeur de quelques expressions; sans cela, il est diffieile de désigner les parties dont on entend parler. Ce sont même les observations qui m'ont été faites par des personnes fort au courant de l'anatomie des Mollusques qui m'engagent à faire précéder les faits d'embryogénie que j’ai à présenter par quelques considérations d'anatomie descriptive.

Les branchies, complétement développées, se composent de chaque côté du corps de quatre leuillets disposés près les uns des autres, et parallèles au plan médian qui partage, en deux parties latérales semblables et symétriques, le corps de l'animal. Les choses étant identiques des deux côtés, ce n'est qu'un seul qui nous occupera.

De ces feuillets, deux sont moyens, et placés entre les autres qui sont extrêmes dans cette séric composée de quatre éléments ; eu égard à la ligne médiane dı corps, les uns sont internes, les autres externes.

Les deux moyens sont unis par leurs bords supérieurs avec le corps entre le pied, la masse viscérale et le mantcau: les autres sont, au contraire, libres par le même bord.

[On n'oublie pas que lorsqu'on regarde un . Mollusque acéphale en le posant sur la partie qui correspond à la charnière, on le place sur le dos, que par conséquent on renverse l'animal, et que ce qui doit être en bas paraît en haut ; après cet avertissement, il ne pourra plus désormais y avoir de doute. La deseription qui va suivre se rapporte à l'animal supposé en place et non à l'animal en préparation, ouvert et renversé.]

Les deux feuillets extrêmes, placés en dedans et 'n dehors des moyens restant libres par leurs bords supérienrs, se soudent avee les moyens par leurs bords inférieurs.

()n peut se représenter ees quatre fenillets ainsi soudés deux à 
deux comme n'étant autre chose que deux lames ployées sur le milieu de leur longueur, l'une en dedans, l'autre en dehors.

De telle sorte que les branchies seraient composées de lames directes ou descendantes (feuillets moyeus adhérents au corps par le bord supérieur) et de lames réfléchies ou ascendantes (feuillets extrêmes, l'un interne, l'autre externe, libres par leurs bords supérieurs, adhérents aux deux précédents par leurs bords inférieurs).

Il n'y aurait done que deux branchies de chaque côté, l'une interne, l'autre externe, représentées chacune par un feuillet ascendant à bord supérieur libre, et un feuillet descendant à bord supérieur adhérent.

Les feuillets moyens seraient les feuillets descendants, directs, adhérents. Les feuillets extrêmes seraient les feuillets réfléchis, ascendants, libres.

Il nous arrivera donc, dans la description, de dire la branchie interne ou la branchie externe pour désigner deux feuillets ; puis, ponr l'une ou l'autre, nous emploierons les épithètes de lame directe, lame réfléchie, ou encore lame adhérente, lame libre, ou bien enfin lame descendante, lame ascendante.

Cette sorte de glossologie est nécessaire; lixer la valeur des termes est indispensable soit pour abréger les deseriptions, soit enfin pour s'entendre et désigner suffisamment les objets dont il est question.

Les lanes sont constituées par des filaments eylindriques, qui dirigés parallèlement les uns aux autres descendent directement, et se placent perpendiculairement à l'axe du corps. Ces filaments sont tenus en rapport les uns avec les autres par des traverses peu nombreuses, perpendiculaires à leur direction, (jui, du reste, n'ont pas pour nous un grand intérêt, et par de véritables articulations ciliaires mobiles qui peuvent ètre rompues, mais ıui se reproduisent bientôt.

Ce dernier fait est assez curiens, et je ne le vois point signalé.

Le long des baguettes qui forment les lames, on rencontre des tubercules hérissés de cils vibratiles courts, dont les mouvements ne sont point semblables à ceux du reste des filaments, et ne déterminent pas de courant. Ces tubercules se correspondent sur. Jes 
divers filaments, ils se font face, de telle sorte que les cils. qui les couvrent en s'agitant, s'enehevêtrent les uns les autres, et restent unis par leurs ondulations. Lorsqu'un effort vient à éloigner deux filaments voisins, les cils sont séparés, et les articulations mobiles ciliaires sont détruites; mais quand l'effort a cessé, on voit bientôt les houppes de eils se pénétrer de nouveau, et reformer ces singuliers moyens d'union.

La disposition de l'appareil respiratoire est loin d'ètre toujours aussi simple dans toute la classe des Acéphales lamellibranches. Il y a même des cas où l'on ne reconnaitrait plus les branchies à liı description qui vient d'être donnée; mais, avec un peu d'attention. on voit bien vite que le plan d'organisation est cependant le même. Dans beaucoup d'espèces, les Unio, les Anodontes, les Bucardes, les Huîtres, etc., etc., les bords supérieurs des feuillets rélléchis sont soudés, l'interne avec celui du côté opposé les externes avec le manteau. De telle sorte que l'on ne voit plus les branehies séparées des deux côtés, et que la masse viscérale semble enlerméc dans l'organe de la respiration, lequel se présente alors comme formé de plis étendus d'un lobe du manteau à l'autre.

Mais ces soudures, qui sont quelquefois très solides et très résistantes, comme dans l'Huitre, l'Anodonte, etc., sont ì peine marquées dans quelques espèces, et il est des Bucardes sur lesquelles on peut très facilement, par une légère traction, rompre l'adhérence des feuillets réfléchis externes avec le manteau; on replace alors cette partie de l'appareil de la respiration dans les conditions que l'on vient de voir dans la Moule.

La lame réfléchie interne se soude par son bord supérienr avec celle dı côté opposé, et forme un sillon sur la ligne médiane. Souvent eette soudure ne se voit qu'en arrière de la misse viscérale, comme dans les Bucardes, les Unio; dans ce cas, on renontre quelquefois les bords supérieurs unis avee le côlé de la sse viscérale en avant; mais bien souvent cette soudure est si peu solide que la moindre traction peut la rompre. C'est ce qu'on peut observer dans les Bucardes.

I a sondure des deux bords supérieurs des deux lanes réfléchies internes existe sur tonte lit largeur dans les Anomies, et on la 
détruit assez facilement; mais, au premier abord, quand on considère les branchies de cet Acéphale irrégulier (1), on éprouve une certaine difficulté à reconnaitre ce même plan que dans les autres animaux. Cependant toutes les adhérences étant rompues, les branchies se retrouvent constituées comme dans la Moule. Ce fait est important, car il permet de supposer que le développement doit ètre sinon entièrement semblable, du moins à peu près le même dans tous les cas.

Dans l'Anodonte, l'Unio, l'Huitre, etc., les branchies semblent bien plus résistantes que dans les espèces que je viens d'indiquer; e'est que des filaments transversaux, unissant et tenant rapprochés les filaments verticaux, sont très nombreux et plus résistants que dans les espèces précédentes, que dans la Mlonle, où ils sont assez rares, et que dans la coquille de Saint-Jacques, où ils sont si peu nombreux, s'ils existent, et si faibles, que la branchie peut à peine ètre touchée sans se décomposer en une multitude de filaments. Dans cette espèce, dı reste, on trouve les feuillets réfléchis avec leurs bords supérieurs complétement libres, et avec une disposition tout à fait semblable à celle que l'on observe dans la Mloule.

III.

MEURS DES EMBRYONS.

Les branchies n’apparaissent dans la Moule que lorsque l'animal a acquis déjà un certain degré de développement. Leur présence doit certainement marquer une période de la vie embryounaire. Dans quelques cas, sur les Huîtres par exemple, j'ai en vain cherché à voir cette période; tous mes efforts ont été vains et inutiles. C'est qu'évidemment elle ne commence que lorsque certaines conditions se présentent. Quelles sont-elles? Je n'en sais rien; je n'ai pas pu encore les saisir; mais à coup sùr personne n'a vu sur cet animal le développenent de la branchic. Je le montrerai surabondamment.

Quant à la Moule il n'en est pas de même; des circonstances

(1) Voir le travail que j'ai publié sur l'Anomie, Amn, des sc. nal., $4^{\mathrm{e}}$ série, 1. II, p. 1 . 
heureuses m'ont permis de me procurer en grand nombre, et à des degrés très divers, les embryons. Aussi ai-je pu suivre l'appareil de la respiration depuis son commencement jusqu'ì son entier développement.

J'avais été frappé, et tout le monde l'a été, sans doute, par l'innombrable quantité de jeunes Noules qui, dans certaines localités, viement se placer sur les bords de la mer presque à lleur d'eau, quand les rochers leur fournissent un lieu abrité où elles peuvent vivre sûrement et tranquillement. Il suffit d'avoir fait une promenade sur les jetées qui servent de cales dans différents ports, dans celui de la Joliette, par exemple, à llarseille (1), pour avoir vu un liséré noirâtre presque à fleur d'eau composé de Moules prodigieusement nombreuses, et serrées les unes contre les autres.

Je me demandais, dans une excursion que de Marseille j'avais faite à la Venise provençale, aux Martigues, si je trouverais sur les bords de la petite mer de Berres la même chose, et je ne tardai pas, en suivant les berges qui sont derrière la ville, près de la route de Marseille, à trouver les Moules en nombre vraiment considérable; mais, en continuant mon excursion, j'arrivai à un endroit où les fucus, sous forme d'un duvet fin, couvraient les pierres. Je cherchai au milieu de ces plantes, et je ne trouvai plus les Moules. Elles semblaient avoir abandonné le bord, gênées qu'elles étaient par la végétation; elles reparaissaient dès que le rocher devenait de nouveau à nu. Il me parut évident que, dans les points les plus voisins de la surface de l'eau, il y avait une grande quantité d'animaux toujours jeunes, alors qu'on n'en trouvait pas de grande taille; qu'il devait y avoir une émigration des individus les plus forts vers des régions plus profondes, et que, à un certain moment, les jeunes Moules, avant de se fixer, venaient vivre à la surface de l'eau, portées instinctivement vers un milieu où l'air et la lumière étaient en rapport avec les besoins de leur activité vitale. Je pensai done qu'on pourrait trouver des larves ou des embryons sur les bords des berges, et je me mis résolùment

(1) L'observation dont il est ici question a été faite dans le mois de septenbre 1853 ; je ne sais si les faits que j'indique ont cessé d'exister. 
à leur recherche. M. Löven n’avait-il pas été la loupe à la main recueillir des embryons sur les fucus, en suivant la marée dans les petites flaques d'eau que laissait la mer?

J'explorai done avec soin les anfractuosités des rochers qui avoisinent le bord de l'eau et qui sont remplies, quand la mer de Berres agitée vient se briser sur le rivage.

Dans ces sortes de petites mares, la faune et la flore présentent les mêmes caractères que dans la mer mème, et je pensai que là, si je devais le reucontrer, je trouverais plus lacilement ce que je cherchais. J'examinai toutes les plantes, et bientôt je reneontrai sur un fucus filamenteux transparent, d'un gris jamàtre, des petits points brunâtres qui me parurent être des animaux fixés. J'avone que jétais loin de penser que j’avais atteint mon but. Quand j'observai les brius de fucus que j’avais récoltés tous chargés de ces points noirs, je ne tardai jas ì reconnaître de jeunes Moules; ear sur une même touffe je rencontrai bientôt des individus de tout àge, et faisant le passage contre l'adulte et l'enbryon.

Ceux qui ont exploré les bords de la mer se feront seuls une idée exacte du plaisir que j'éprouvai en trouvant ainsi une mine aussi riche et inépuisable, qui, à la porte mème des Martigues, pouvait me fournir un sujet de travail sans perte de temps. Je fus donc bientôt installé et à l'œuvre.

Je n'avais garde de laisser échapper une telle occasion; dans l'étude de l'embryogénie des Acéphales comme dans celle des autres Mollusques, toute la question est d'avoir des embryons. Car rien u'est diffieile comme de les élever; pour beaucoup d'espèces nême c'est la seule cause du retard qu'éprouvent nos connaissances.

Je me plais à signaler aux naturalistes la circonstance heureuse, dont je me hàtai de profiter autant que le temps me le permettait; car, pour les autres points de l'histoire embryogénique de la Moule, on trouvera à coup sùr, dans la localité que j’indique, lous les matérianx nécessaires. Il suffit en ellet, du mous dans l'année et à l'époque dont je parle, de chercher à l'est des Nartigues, en se dirigeant vers Saint-llédard, pour reneontres des fuantités 
innombrables d'embryons de $1 / 4$ à $\mathbf{1} / 5^{\circ}$ de millimètre de diamètre, et de toutes les grandeurs au-dessus.

Mes observations ont été faites dans la première quinzaine de septembre, une amnée où la saison, pluvieuse et froide, avait fort retardé le printemps. Il serait curieux de faire des observations dans le courant de l'été, pour voir à quel moment lesjeunes Moules se fixent anx fucus, et en quel état elles sont quand elles viennent ainsi à la surface de l'eaur.

Serai-je assez heurenx pour pouvoir moi-même faire les recherches que j’indique? Si mes occupations me le permettent, à coup sur je reverrai les Martigues, et j'exploiterai de nouveau les heureuses conditions dont je viens de parler.

On ne saurait trop multiplier les indications qui, plus tard, penvent ètre d'un précieux secours aux naturalistes. Les auteurs qui écrivent, ou qui écriront sur l'embryogénie des animaux marius, ne doivent rien négliger pour faire connaitre les moindres particularités de mocurs. On ne saurait croire combien, pour le zoologiste voyageur, sont utiles et importantes les plus simples indications.

Aujourd'hui que les études d'anatomie physiologique sur les bords de la mer sont poussées avec lant d'activité, il y aurait tout un livre à entreprendre pour faire connaitre la géographie zoologique marine, non-seulement pour des pays éloignés, mais encore pour une même localité.

Ainsi, pour ne citer (qu'un exemple, il n'est pas douteux que sur les plages que découvre la marée basse, on ne trouve des animaux à des profondeurs diverses. La Moule n'atteint pas de grandes profondeurs; c'est ce que l'on peut constater sur toutes les côtes de la France avec la plus grande facilité.

Les pêcheurs riverains, je dirais mieux les populations côtières qui suivent la marée pour butiner après elle, savent très bien quiils ne trouveront tel ou tel animal que lorsque la grève découvrira jusqu'à telle ou telle profondeur. Ils se préparent d'avance pour telle marće, parce que c'est alors qu'ils feront une plus riche prise.

Je pourrais citer des exemples nombreux. Des animaux qui m'ont fourni des sujets d'étude se trouvaient seulement à certaines 
profondeurs et non pas à d'autres; je connaissais sur des banes de sable des zones où à coup sûr je rencontrais des Dentales, et si je les trouvais ailleurs, c'est que la mer les avait roulés et déplacés.

Les animaux des côtes habitent done comme des zones spéciales. On sait aussi que beaucoup n'abandonnent jamais la haute mer; enfin que ces zones peuvent changer avec l'âge : c'est ce qui arrive en particulier pour la Moule. Sans aucun doute elle vient à la surface de l'eau cuand elle est encore embryon, et c'est là ce qui explique ces banes de Moules qui, dans la Médilerranée, bordent les rochers à fleur d'eau.

Des observations directes m'ont encore fourni la preuve de ce dernier fait. Quand je plaçais, dans de l'eau très pure et parfaitement renouvelée, les fucus clrargés de petites Moules, constamment les embryons gagnaient le haut du vase, et formaient à la surface de l'eau comme une couche noirâtre. Il y a done dans cette étude des mœurs des animaux d'intéressants sujets de recherches qui serviraient plus tard de guides précieux pour les excursions marines de physiologic et d'anatomic.

Je n'ai point observé les jeunes Moules avant qu'elles fussent fixées; mais je crois que par analogie on pourrait, d'après ce que M. Löven, M. de Quatrefages et moi-même avons vu sur d'autres espèces, admettre que la Noule vient ainsi à la surface, portée qu'elle est par son appareil locomoteur ciliaire, son disque moteur, comme dans les autres espèces d'Acéphales lamellibranches, et qu'alors son byssus, trop délicat encore, ne suffit pas pour la fixer sur les rochers où les mouvements des vagues sont trop forts. Aussi, sur le bord de l'étang de Berres, je reconnaissais, guidé par ces données, les points abrités où je rencontrerais de jeunes embryons en grande quantité. Je m'explique pourquoi aussi, sur la jetée du sud du port de la Joliette, les Moules abondent : elles ont pu s'y fixer à l'abri des coups de mer.

Ce voisinage de la surface est évidemment en rapport avec les besoins de l'animal; car dans les moments d'agitation de l'eau, quand la jeune Moule est fixée, soit aux fucus, soit aux rochers, les vagues en se retirant la laissent à découvert ; elle est exposée à l'air, condition qui évidemment ne lui 'est pas défavorable. 
J'ai fait, disais-je, mes olsservations au commencement de septembre; bien que cette année-lì la saison fût retardée, il est peu probable que les embryons de 1/4 de millimètre, les plus petits quiil m'ait été donné de rencontrer, fussent du mois d'avril. Il me semble qu'ils devaient être moins âgés. On sait que Baster (1) et Poli (2)étaient en désaccord sur le moment de la ponte des Moules. Le premier assignait les mois d'avril et de mai, le second le mois d'octobre jusqu'en décembre. Ces deux observateurs entendaient parler des Moules des côtes de la Hollande et de la mer de Tarente, et je crois qu'ils devaient l'un et l'autre être dans la vérité (3). Ne serait-on pas tenté de croire, d'après la taille des jeunes embryons que j'observai aux Martigues, qu'il peut, pour des points intermédiaires aux localités précédentes, y avoir aussi des époques intermédiaires pour la ponte?

Je n'ai pas observé l'embryon à partir de l'neuf; c'est done là une nouvelle recherche à faire pour reconnaître l'époque de la fraie dans la localité dont il est ici question. Cette époque, du reste, doit être bien connue des pêcheurs (4) de Moules des Martigues, où ce Mollusque, très abondant, donne lieu à une pêche fort active, qui fournit des bénéfices considérables, car il est estimé.

\section{IV.}

\section{ORGANISATION DES EMBRYONS.}

Voyons maintenant quelle était l'organisation des jeunes Moules au moment où j'ai commencé les observations.

Sur les plus jeunes embryons, l'anatomie peut se faire par trans-

(1) Poli (Xav.-Jol.). Testacea utriusque Sicilice. Paris, 1791-1795.

(2) Baster (Job.). Opuscula subseciva, continentia observationes miscellaneas de animalculis et plantis quibusdam marinis eorumque ovariisque et seminibus, 2 vol. in-4. Harlemi, 1759-1765.

(3) Voir les observations que j'ai publiées sur les organes de la reproduction des Mollusques acéphales lamellibranches. - Considérations générales et historiques. (Ann. des sc.nat., t. II, $4^{\mathrm{e}}$ série, p. 155 .)

(4) Je vois que, dans les notes prises dans la localité, j'ai omis de signaler exactement le moment de la ponte; je préfère donc m'abstenir plutôt que de me confier à mes souvenirs. 
parence; mais, dès que les appareils ont pris quelque développement, il faut arriver ì des dissections très minutieuses, le plus souvent fort laborieuses, que j’ai cependant pu conduire ì bonne fur, sous l'excellent microscope ì dissection de M. Nachet, avec le trépied mobile à miroir qui sert de table.

Test.

La corjuille dans les jeunes Noules est entièrement différente de ce qu'elle sera plus tard chez l'adulte, puisqu'elle est presque ovale, et que la charnière ainsi que le ligament se trouvent vers le milieu du grand diamètre. I re moment, on pourrait comparer sa forme ì cellę d'une Mactre, en petit toutefois (1). En avant et en arrière dı ligament élastique (2) sont des dents très nettement dessinées, et l'engrènement (ju'elles forment(3) est toujours facile à constater.

On remarque sur les deux valves des stries circulaires peunombreuses, qui, dans les individus de très petite taille, sont d'autant plus rapprochées d'une circonférence régulière qu'elles sont plus centrales, et voisines du crochet. Cela montre évidemment que, dans le commencement, les valves du test étaient arrondies, et que leur forme ne s'est allongée que par les progrès du développement; ct plus tard, fuand la coquille sera piriforme, on ne pourra expliquer cette nouvelle apparence que par l'accroissement disproportionné que prend le diamètre longitudinal antéro-postérieur, comparativement au diamètre transverse ou vertical, surtout en arrière de la charnière. Il semble, sur la coquille d'un adulte, que le sommet des crochets qui se trouve presque au-dessus de la bouche soit formé par les premières couches testacées de l'embryon.

Du côté dı dos, les valves présentent les deux crochets, qui peu à peu portés en avant, laissent en arrière la portion postérieure du test, et logent les deux lobes du foie.

(1) Voyez t. V, pl. 2, fig. 2, 3, 4.

(2) Idem, fig. $2(l)$.

(3) Idem, fig. $2(\mathrm{ch}, \mathrm{ch})$. 
Eı regardant l'animal de champ (1), on aperçoit la charnière, qui, reetiligne dans toute la partie moyenne correspondant aux crochets des valves, devient sinueuse en avant et en arrière. On y remarque (3) cind dents du còté de la bouche et trois seulement en arrière, s'engrenant les unes les autres avec une grande précision. La r'́union des deux valves est complétée par un ligament élastique bien évident, 'łui semble formé de deux moitiés semi-lunaires (4), et qui est plus rapproché des dents postérieures que des antérieures.

Un fait rui m'a paru singulier est celui-ci : Les dentelures s'effacent de plus en plus, ì mesure que les progrès du développement avancent davantage, ef lorsque les branchies sont complétement constituées, il m’a été impossible de les distinguer. L'épaississement des parties les aurait-il cachées et dérobées à l'observation?

Il n'est pas possible de comparer complétement les jeunes Ioules dont il vient d'être question aux larves des Huîtres, même les plus développées, et dont j’ai présenté ailleurs une partie de l'histoire (5); car on établirait un parallèle entre des êtres qui ne sont point dans des périodes correspondantes de leur développement. Mais dans les jeunes Huitres cependant, on trouve une charnière qui présente beancoup d'analogie avec celle-ci.

Les valves de la jeune Moule sont tenues rapprochées, par un musele (6) bien développé situé en arrière; on voit autour de lui le rectum, et dans son voisinage l'anms. Le muscle correspond évidemment à celui qui, dans l'adulte, est postérieur. N'ayant pas observé celui qui est du côté de la bouche en avant, mais M. Löven l'ayant indiqué dans les figures des jeunes Acéphales dont il a fait l'histoire, je crois qu'on peut arlmettre que le musele postérieur est le premier à se développer, et que par couséquent les Noules qui

(1) Voyez pl. 2, fig. $(3,4)$.

(2) Voyez pl. 2, fig. (2).

(3) Voyez pl. 2, fig. (ch, ch).

(4) Voyez pl. 2, fig. (l).

(5) Voyez Comptes rendus de l'Acudemie des sciences de l'Institut de France, t. XXXIX, année 1854, p. 1197.

(6) Voyez pl. 2, fig. 3 et 4, dans le voisinage d'un organe marqué $r$. 
sont dimyaires commencent par être d'abord monomyaires; fait qui pourrait expliquer, par la théorie des arêtes de développement, comment certains Mollusques, tels que l'Huitre, la Coquille de Saint Jacques, le Spondyle, ete., restent toujours monomyaires.

\section{Manteau.}

A l'époque où nous étudions la Moule, son manteau présente déjà la disposition que plus tard il aura ehez l'adulte, mais cependant à undegrémoindre. Les deux moitiés, ou lobes, sont soudées sur la ligne médiane, en arrière, du côté opposé à la bouehe (1).

La soudure, dans les plus jeunes individus, remonte assez hant ; elle embrasse la base dı pied qui semble sortir de son intérieur $(\mathbf{2})$ par une ouverture spéciale. En arrière, l'union des deux lobes est interrompue à la hauteur de l'anus : e'est le commencement du tube postérieur respiratoire. Restée à peu près la même pendant que les autres parties croissent, la soudure devient relativement plus petite, et s'éloigne de la base du pied; aussi présente-t-elle une ouverture antérieure d'autant plus grande, que l'animal est plus avancé en àge. On voit au travers de cette fente les filets branchiaux (3); en arrière d'elle, vers la base, on remarque aussi une languette qui, en se rabattant, peut jouer le rôle de valvule ; c'est le rudiment de ee qui existe sur l'adulte, et qui est si prononcé dans quelquesf espèces : les Lithodomes, les Bucardes, etc.

Est-il besoin de dire que le manteau est très sensible, très conIractile, et qu'il est eouvert de cils vibratiles nombreux que l'on distingue faeilement?

\section{Pied.}

Cet organe, destiné spéeialement à la locomotion, présente déjà un développement considérable au moment de l'apparition des branchies, et, pendant la formation de eelles-ci, il eontinne son accroissement.

Quoirquecylindroïde, il mérite hien cependant son nom, car il sert

(1) Voyez pl. 2, fig. 1 (m).

(2) Voyez pl. 2, fig. $1(p)$.

(3) Voyez pl. 2, fig. I (br.). 
beaueoup à l'animal; recourbé halituellement en avant, il se porte. dans tous les sens; son extrémité libre est un peu évasée, surtont dans les moments où l'animal l'applique sur les corps en manière de ventouse. Cettc extrémité présente, en effet, une exca. vation, qui se continue en forme de sillon tout le long de la face inférieure, comme, du reste, dans l'adulte (1).

Le jeune animal s'en sert à peu près comme les Sangsues se servent de tout leur corps pour la locomotion; il le porte au loin dans tous les sens, et semble tâter, palper les objets; puis ille fixe dans le point choisi, et alors, en se contractant, il peut rapporter, attirer et ramener son corps vers le point où avait eu lieu l'adhérence de la ventouse. J'ai suivi bien souvent, sous le microscope, les jeunes Moules rampant ainsi sur les brins de fucus. Quand je les plaçais dans des vases de verre, je les voyais, ì l'aide d'une bonne loupe, avancer sur les parois à la manière qui vient d'être indiquée. La locomotilité de ces jeunes larves est encore assez grande, et l'on peut ainsi les voir se déplacer jusqu'au moment où elles se fixent par leur byssus.

Du reste, ce mode de progression n'appartient pas seulement aux jeunes Moules. La Cyclas (Cyclas cornea), que l'on rencontre en quantité prodigieuse dans les environs de Lille, se déplace avec une très grande facilité au moỵen de son pied, qu'elle étend et fixe en manière de ventouse. On n'a qu'à placer dans un vase de verre ces Aréphales pour les voir ramper (l'expression est très juste), et monter jusqu’à la surface du liquide, et si, dans le vase, se trouvent quelques filaments de Conferves, on les voit bientôt s'y attacher et s'y suspendre toujours à l'aide de leur pied.

C'est à ce mode de locomotion que je dois d'avoir pu examiner l'animal de face. En plaçant la jeune Moule dans des cuvettes à faces planes, parallèles, formées de lames de verre excessivement minces, et spécialement disposées pour l'examen des petits êtres, on la voyait ramper tantôt sur la face inférieure, tantôt sur la face supérieure, et présenter dans un cas le dos, dans l'autre la partie inférieure.

(1) Voyez pl. 2, fig. $1(p)$. 
Le pied est couvert de cils vibratiles très vifs, au milieu desquels on rencontre des cirrhes ou cils plus grands, également fort mobiles; tous servent à la locomotion. Quand l'animal veut nager, il étend son pied, et il avance par les mouvements seuls des cils. Ce moyen est employé de moins en moins, ì mesure que l'animal est de plus en plus développé.

On voit dans le pied une cavité générale, où les contractions agitent des globules.

\section{Byssus.}

Quand on place dans un vase de verre une touffe de fucus chargée de petites Moules, bientôt les parois se couvrent d'une quantité considérable d'embryons. Alors on peut changer l'eau, renverser le vase, sans que ceux-ci se détachent. L'adhérence a lieu au moyen d'un byssus, qui ne peut être mis en doute sur des individus de $1 / 2$ millimètre, mais qui est plus difficile à constater sur les individus de plus petite taille. Dans ces derniers, il faut apporter toute son attention pour reconnaître l'organe filamenteux qui sert à les lixer. Il n'est composé que de deux, trois ou quatre fils trausparents hyalins, parfois ramifiés, et qui naissent de la face inférieure du pied, tout près de sa base, dans la fente ou scissure même que l'on voit sur la face inférieure (1).

Il m'a semblé, mais je dois avoucr que l'observation offre de grandes difficultés, que les filaments du byssus étaient de petits iubes, dans l'intérieur desquels était comme une matière plastique 'jui, lorsque le filament venait à être rompu, s'échappait à l'extrémité, et y faisait un petit bourrelet; ce serait cette matière plastique qui se fixerait aux parois.

Plus tard, j'ai eu oceasion d'observer les mœurs d'une Moule rose que j'avais trouvée sur les côtes du Bretagne, et j'ai pu voir comme une sorle d'épanouissement de tubercule blanchàtre terminant chaque filament. J'avais placé la Moule dans un vase de terre verni en noir; elle avait changé plusieurs fois de place en cassant les tubes de son byssus, on voyait de petits disques qui venaient se former ì l'extrémité de chacun de ees tilaments, à mesure qu'elle

(1) Voyez pl. 2, lig. 1. 
se fixait de nouveau. On pourrait done avoir l'idée du ròle de l'organe qui nous oceupe, en le eonsidérant tout simplement eomme IIII faisceau de tubes destinés à porter dans le point, où doit se fixer l'animal, la matière plastique destinée à cet usage.

\section{Organes internes.}

A part les branchies que je laisse de côté, il n'y a que l'appareil digestif de lormé dans les jeunes Moules dont il vient d'être question. Des organes qui servent ì la conservation de l'individı, c'est, en effet, celui de la digestion qui se développe le premier après celui de la locomotion.

Nous devons étudier la bouche, l'estomac, l’intestin, l'anus, le foie.

La première est fort difficile ì voir, et cela s'explique. En avant du corps (1), il y a une sorte de dépression, où bien certainement est la bouche, mais qui est remplie le plus souvent, quand l'animal est en repos, par le pied qui s'y retire comme dans une loge ou uII abri. Aussi n'ai-je jamais pu observer nettement cet orifice, dont la position dans un point de cette partie antérieure ne pent ìtre douteuse.

Comme, dans presque tous les Acéphales lamellibranches, l'estomac est placé dans le voisinage de l'orifice d'entrée, c'est à peine si le tube qui y conduit peut recevoir le nom d'osophage. (Mn reconnaît bien vite l'estomac à ses parois épaisses, écartées, limitant une eavité étendue, au milien de laquelle paraissent tourbillonner des globules plus ou moins colorés, qui sont tantôt de petits corps avilés far l'animal, tantôt des particules de malière colorante fournie par le foie. Les mouvements de ces petites masses sont très évidents, tant que l'animal est bien portant; ils cessent dès que la vitalité paraît un peu se ralentir, ct alors il n'y a plus cette ligne hien nette et transparente entre les parois et le milieu de la ca-

(1) Voyez V, pl. 2, fig. 2 et 4 . On voil le pied qui se recourbe en haut, et dont la pointe est rapprochée du crochet el du foie. - De même dans la figure 3. 
vité (1). Dans les jeunes Huìtres, ce phénomène me guidait toujours dans l'appréciation que j'avais à porter sur la durée de l'existence des jeunes animaux que j’élevais.

L'estomac se contracte aussi de temps en temps, comme, du reste, les autres parties de l'organisme.

L'intestin offre les mêmes caractères que che\% l'adulte; mais ces caractères sout d'autant plus marqués que l'on avance davantage; ainsi, à mesure que les branchies se développent, on voit les circonvolutions intestinales plus nettement dessinées.

L'estomac se rétrécit vers son extrémité postérieure dans un point voisin du muscle postérieur des valves, et à son rétrécissement fait suite l'intestin qui remonte vers le dos, pour se diriger d'arrière en avant, un peu sur le côté de l'estomac. A gauche, après être arrivé eli avant, presque à la naissance de la cavité stomacale, il redescend en longeant la première anse et restaut à son côté gauche ; puis se rapprochant de nouveau de la ligne médiane, il gaçne la face dorsale du muscle des valves, se contourne pour venir s'ouvrir, comme dans tous les Acéphales, au-dessus et en arrière de lui.

Quand on peut arriver à fixer l'animal ou à l'empêcher de se mouvoir trop librement, on distingue très nettement les cils vibratiles dans l'intérieur dı tube intestinal oì ils déterminent un courant assez rapide.

Enfin le foie est de tous les organes celui que l'on aperçoit le plus promptement et avec le plus de facilité ; car il frappe immédiatement par sa couleur brunâtre à la fois jaune et un peu verlàtre. Il est formé de deux masses, l'une droite, l'autre gauche, dont le volume est très differrent; l'un et l'autre occupent les crochets de la coquille, mais dans des proportions variables. Le côté droit (2) s'étend beaucoup moins en arrière; il semble sphéroïdal ; celui de gauche, an contraire, est comme bilobé (3). Plus

(1) Zool., pl. 2, fig. $2(g)$, fig. $3(g)$.

(2) Voyez pl. 2, fig. $3(f)$, fig. $2(f)$.

(3) Voyez $\mathrm{pl}$. 2, fig. \& (I), fig. 2, où l'on peut bien apprécier la différence des formes des deux lobes. 
lind, l'estomac et l'intestin seront perdus pour ainsi dire an milien de la masse glandulaire, qui s'étendra toujours davantage à gauche ' fu'à droite. La position de l'estomac, plus de ce côté que de l'autre, explique certainement la prédominance du lohe droit relativenent all lobe gauche.

On distingue dans le milieu de ces lobes comme un éclairci; la substance glandulaire semble moins épaisse en ce point; cela tient ıniquement à la position du microscope. Quand on place les pourtours au foyer, le centre parait transparent, parce pu'il y a une cavité an milieu de la masse glandulaire qui communique avec l'estomac; ces cavités plus tard, en suivant l'accroissement de la glande, s'allongent et finissent par former des camaux biliaires, toujours extrêmement larges, et dilatés dans les Mollusques Acéphales.

On voit même dans cetle cavité les mouvements ciliaires; c’est qu'en effet, comme je le montrerai plus tard dans un travail que je me propose de publier bientôt sur la structure du foie des Acéphales, les canaux biliaires, qui sont tapissés par la substance glandulaire elle-même, sont revêtıs par un épithélium vibratile, qui s'étend jusque dans le fond des culs-ile-sac sécréteurs.

Tels sont les principaux organes de la digestion; on voit yu'ì l'époque où nous allons prendre le développement de la jeune Moule, ils sont assez nettement dessinés. Eux et les organes de la locomotion sont les plus parfaits; ils ne peuvent ètre confondus aver aucun autre.

II. est encore deux choses que j’ai observées non pas dans les embryous ayant déjà des rudiments de branchies, mais dans ceux qui avaient déjà pris un peu plus de taille, bien que cependant ils n'eussent pas encore un grand nombre de filanents respiratoires.

En avant de la première baguette des branchies, en arrière de la partie antérieure du lobe gauche du foie, et à la base du pied, j’ai aperçune petite vésicule(1)sphérique, dans l’intérieur de laquelle

(1) Voyez Zool., pl. 7, fig. 4 (o). 
s'agitaient, d'un mouvement analogue à celui que l'on appelle mouvement brownien, de tout petits corpuscules toujours placés an centre. M. Löven a vu et dessiné cette même capsule dans la Moule plus développée; c'est, à n'en pas douter, une capsule auditive; ce sont les otolithes de M. Von Siebold. La position à la base du pied avec les autres dispositions la caractérise suffisamment.

Enfin dans le point où l'on rencontre habituellement le cour et les organes de Bojanus chez l'adulte, on voit une vésicule transparente, hyaline, à peine appréciable par un contour léger. Elle a été vue aussi par M. Löven, qui a signalé, même dans son intérieur, l'existence de cellules, ce qui ne permet guère de ne pas reconnaitre en elle l'origine dusae de Bojanus. Cette vésieule paraît entre le lobule postérieur du foie, le muscle postérieur des valves en dessous du tube digestif (1). Je dois me ranger à l'opinion de M. Löven ; car dans les jeunes Cyclas j'ai vu la même chose ; et déjà dans cet Acéphale la substance earactéristique se fait reconnaitre. Il est vrai lle dire anssi que dans ces derniers embryons le cœur battait.

J'avais eu une autre opinion, je dois l'avouer; car j'avais vu ces vésicules se contracter à de très longs intervalles, il est vrai ; j'avais d'abord pensé qu'elles devaient représenter les oreillettes. Mais comme, sur des individus beaucoup plus développés, il m’a été impossible de voir le coeur, j’ai dù abandonner cette opinion, surtout quand plus tard j'ai connu ce que disait M. Löven dans son remarquable Mémoire : "Je n'ai jamais été assez heureux "pour l'observer (le cœur) chez aucun Acéphale examiné, et je " désespère même de le faire. "

Si j'insiste sur ce déreloppement du cour, e'est que personne u'a encore vu naître cet organe dans les Aeéphales. Les larves de jeunes Huîtres, quej’aićlevées et conservées pendant quarante-trois jours après les avoir extraites du manteau de leur mère, ne m'ont pas fourni l'occasion de voir se former l'organe central de la circu lation, el elles n'avaient point de branchies.

1) Voyez pl. 7, fig. 3 et $4(r)$. 
En résumé, la jeune Moule, quand ses branchies vont paraìtre, ne possède que des organes de la locomotion et de la digestion. Quand l'appareil de la respiration se montre, le pied est nettement dessiné; il est pourvu d'un byssus. Les otolithes sont appréciables ainsi que les rudiments de ce que $M$. Löven considère comme l'organe de Bojanus. Le tube digestif se compose d'un estomac et d'un intestin forts distinets, et le foie est nettement divisé en deux lobes, qui sont chacun creusés d'une cavité débouchant dans l'estomac.

Il n'y a, comme on le voit, ni cour, ni système nerveux proprement dit, ni à plus forte raison de glandes génitales.

\section{V.}

DÉVELOPPEMENT.

Malgré tous mes soins, toute l'assiduité de mes recherches, je I'ai pu, à l'époque où je les observais, rencontrer des individus portant moins de trois à quatre rayons branchiaux. Je ne puis done ici indiquer l'origine de ces premiers éléments de l'organe respiratoire; mais, sans foreer beaucoup l'analogie, il est permis de penser que ceux-ci ont été produits absolument comme ceux qui viennent plus tard, et qui se rangent à côté d'eux.

M. Löven n’a pas dépassé le nombre de neuf ou dix rayons; d'après ce qu'il dit même, on eroirait qu'il ne s'est préoecupé que d'une chose: qu'il n'a voulu montrer quelapremière phase du développement : car il affirme que ce qu'il a vu est bien l'organe de la respiration représenté par des baguettes nées sur les eôtés du corps.

Voici ce que nous devons chereher à reconnaitre. D'abord dans quel ordre apparaissent chacun des feuillets, et par quelle série de transformations les trois ou quatre filaments qui ont été observés se sont trouvés remplacés par des lamelles et des feuillets, comme cela a lieu chez l'adulte? En répondant à toutes ces questions, on le voit, le mode de développement des branchies sera complétement démontré. 


\section{Branchie interne.}

Feuillet moyen, direct ou descendant.

Je viens de dire qu'il m'avait été impossible de rencontrer moins de trois rayons. Dans deux des dessins qui accompagnent ce mémoire, on voit (1) des baguettes qui, de chaque côté du corps, s’avancent à l'opposé de la charnière vers le bord libre du manteau et des valves. Ce sont, les premières parties de l'appareil, celles qui constitueront la lame moyenne ou directe de la branchie interne de droite ou de gauche; on voit done que d'abord cette lame commence par être une grille composée de quelques baguettes très distinctes et non soudées entre elles.

On voit aussi, pour m'en tenir à ces exemples, que pour former les fentes ciliées du Taret indiquées par M. de (Quatrefages, il suffirait de raccourcir ici beaucoup les parties, et de rapprocher leurs extrémités jusqu'au contact.

Remarquons aussi que l'extrémité des rayons est plus grosse, et semble renflée en une sorte de tête, qui devient bientôt, quand le nombre est plus grand, comme vaguement trilobée.

M. Löven, je l'ai dit, pense que, chez la Montacuta, un cordon, offrant trois renflements sensibles, sort de la paroi postérieure du manteau. Je ne puis apprécier et juger cette opinion, les observations me faisant défaut; mais pour les autres filaments, la nature suit une marche que je crois avoir reconnue, et qui me parait être la véritable, car elle rend trop bien compte de certaines dispositions.

Voici quelle elle est :

Dans le point où le manteau, le muscle des valves et les viscères, sont unis, un bourgeonnement des tissus a heu, et produit de chaque côté un tubercule qui, en s'allongeant, forme bientôt une baguette. Le premier de ces tubercules a dù se former, sans aucun doute, tout à fait à la base du pied, tandis que ceux quie viennent après naissent et se disposent en arrière, en ligne droite, et dans

1) Voyez fig. (3) (4) (1) (br). 
un plan parallèle à celui qui partage le corps de l'animal en deux moitiés symétriques.

C'est là une supposition, mais tout porte ì croire que cette origine est bien réellement la véritable, si l'on en juge par ce qui se passera plus tard.

A partir du troisième rayon branchial, on voit successivement apparaître les nouveaux éléments de la branchie sous forme de tubercule, de petites élévations, qui peu à peu s'allongent et s'éloignent de celui qui les précède.

La dépression qui sépare ces tubercules ou bourgeons est d'abord marquée en avant (1); plus tard une dépression se forme en arrière ; celle-ci isole le bourgeon du manteau, tandis que la première l'avait isolẻ du filament antérieur (2). Ainsi séparé, le tubercule s'allonge, et de bourgeon qu'il était (3), devient rayon; alors il se place ì côté des précédents, et le nombre des éléments de la branchie se trouve porté de trois à quatre; en arrière du quatrième s'opère un travail semblable : un tubercule se montre isolé en avant d'abord, confondu avec le manteau encore en arrière; bientôt une dépression le sépare de ce dernier, et le nombre des rayons s'aceroît; il est porté de quatre à cinq. Ainsi de suite, le nombre augmente rapidement.

Je ne saurais dire si primitivement il y a un cordon produisant ces tubereules, comme le dit M. Löven; mais quand ils se sont multipliés, leurs bases, unies entre elles, forment un bourrelet longitudinal, qui alors mérite en effet le nom de cordon. Cela devient très évident quand le nombre des rayons branchiaux est porté à neuf ou dix (1); alors aussi les rapports sont tels, entre ceux qui sont les plus anciens el ceux qui paraissent de nouveau, que l'origine par le bourgeonnement ne peut plus faire de doute.

Ainsi les premiers phénomènes, en partant du quatrième rayon branchial, consistent en un bourgeonnement, qui étend la branchie

(1) Voyez pl. 2, fig. $3(a)$.

(2) Voyez pl. 2, fig. $4(a)$.

(3) Voyez pl. 2, fig. $3(a)$.

(4) Voyez pl. 2, fig. 5. - Au bas, de cette figure on voit les bourgeonnements de taille différente qui doivent augmenter le nombre des rayons. 
d'avant en arrière en lui ajoutant postérieurement toujours de nouveaux éléments. N'est-il pas naturel de penser que les trois premiers que je n'ai pu voir naître se sont développés de la même manière?

Pendant ce travail, les tubercules en s'isolant se couvrent aussi de cils vibratiles. Ceux-ci se disposent sur deux séries ou lignes doubles, l'une antérieure, l'autre postérieure. Les différents rayons se portent en bas en se courbant en dedans, et forment par leur rapprochement et leur position dans un même plan une sorte de lamelle percée delongues fentes. Les extrémités se touchent toutes, mais sans être encore soudées; leur union a lieu plus tardivement.

Les rayons un peu courbés en are vers la ligne médiane rencontrent ceux du côté opposé; aussi quand on observe les embryons en dessous, une apparence analogue à celle de la cage thoracique: se fait-elle remarquer (1).

Les extrémités des rayons des deux côtes ainsi rapprochées alternent les unes avee les autres sans qu'il y ait soudure, et quand l'animal sort ou rentre son pied, on voit cet organe s'enfermer sous le grillage, ou s'en débarrasser en écartant les bagıettes qui le forment.

A mesure que les rayons deviennent de plus en plus longs, et qu'ils se dessinent mieux, les cils qu'ils portent se disposent aussi plus régulièrement; ils se placent sur le côté antérieur et le côté postérieur en deux rangées, en tout quatre, deux internes, deux externes. Les mouvements sont inverses sur les côtés de deux rayons voisins. Ainsi supposons que, dans une baguette branchiale, sur son eôté postérieur, le courant soit dirigé de la base d'insertion à son sommet libre, celui que présentera le côté antérieır du filament suivant, sera dirigé inversement de l'extrémité libre à la base d'insertion; il en résultera que les particules paraitraient marcher sur les bords d'un rayon dans un sens, tandis qu'elles s'avancerout en sens inverse sur le bord de l'autre. Quand les mouvements ciliaires sont dans un état convenable, on eroirait voir tout autour de la fente les dents d'une roue dentée d'engrenage; c'est ce que I. de Quatrefages a très bien décrit dans le Taret, et rendu très exactement dans ses dessins.

(1) Vovez pl. 2, fig. $1(b r)$. 
On distingue dans ces filaments un milieu qui semble opaque, plus obscur, entouré d'une couche finement granuleuse, qui se continue avec l'enveloppe du corps. L'une forme le tissu mou de la branchie, l'autre en est la charpente; au milieu se creuse plus tard un vaisseau, qui entre en communication avec l'appareil de la circulation.

\section{Feuillel interne réfléchi ou ascendant.}

Je dois anticiper sur ce qui sera démontré plus tard; je dois dire que la branchie, qui se développe la première, est la branchie interıe. Chaque branchie est considérée, on se le rappelle, comme ayant deux feuillets; le second feuillet de la première branchie, de celle dont nous étudions le développement, se forme avant que la branchie externe ait commencé à paraître. Ainsi done, l'ordre de succession est celui-ci :

$1^{\circ}$ Le premier feuillet ou feuillet direct descendant de la branchie interne;

$2^{\circ}$ Le deuxième fenillet ou feuillet réfléchi, ascendant, de la mêne branchie.

On verra plus loin quelle conséquence remarquable on peut tirer de cette sorte de retard.

Dans le développement que nous allons étudier maintenant, nous trouvons une marche toute différente de celle que nous venons de voir, car le deuxième feuillet s'aceroît en sens inverse du premier.

Il ne commence à être appréciable que vers le moment où la première lame présente de neuf ì onze rayons branchiaux bien développés, sans y comprendre les hourgeons ou tubereules en voie de formation, toujours au nombre de trois ou quatre $(2)$.

Jusque là, on pouvait observer les jeunes Moules par transparence et sans préparation. Maintenant il n'en est plıs de même, et l'on doit avoir recours à des dissections, qui deviennent d'une grande difficulté. Elles consistent à séparer avec deux aiguilles,

(1) Loc. cil., Embryogénie du Taret.

(2) Voyez. pl. 2. fig. 5. 
sous la loupe, les deux valves de la coquille, puis à dissocier les parties molles, respectant autant que possible les branchies déjà formées. Quand on peut arriver à ce résultat, on voit sur le côté interne que les extrémités renflées des rayons, qui avaient été d'abord libres, se sont soudées, et ont formé une lame, mais une lame percée de longues boutonnières. Cette soudure des extrémités inférieures des rayons branchiaux produit comme un bourrelet, comme un cordon (1), qui limite en bas la branchie, comme nous avions vu un cordon la terminer en hant vers son insertion au corps.

Ce dernier cordon est le résultat de l'accroissement des têtes des rayons qui, en s'étendant latéralement, jettent comme un pont entre elles; c'est ce pont qui, en augmentant ì son tour, forme une sorte de repli membraneux (2); pour voir cela, il est nécessaire d'une étude attentive.

La soudure, on doit le prévoir, ne peut commencer sur les rayons les moins développés; aussi ce sont les têtes des rayons les plus anciens qui se réunissent les premières, et sur les branchies de neuf à dix rayons, on voit très neltement les six ou sept têtes antérieures parfaitement unies, tandis que les trois postérieures sont rapprochées sans être soudées; quoique cependant elles se préparent à la réunion. Sur des exemples, dans ces conditions, on voit bien neftement que la lamelle, qui est la conséquence de la soudure, est d'autant plus large, qu' elle est plus rapprochée du côté antérieur. J'ai pu voir ces faits sur des branchies qui n’avaient de soudés que les trois ou quatre rayons antérieurs. Les lames s'étendent naturellement dans des sens différents en largeur et en hauteur. Dans le premier sens, l'aceroissement est semblable pour les deux lames; dans le second, il est inverse. L'une, en effet, descend de haut en bas, l'autre s'élève de bas en haut. Le mode d'accroissement des deux lames est donc en partie le même, en partie différent.

Je laisse de côté pour un instant le mode, suivant lequel les fentes en boutonnière s'effectuent. Il est le même pour les deux

(1) Voyez pl. 2, fig. $5\left(b, b^{\prime}, b\right)$.

(2) Voyez pl. 2, fig. 5 (c). 
feuillets réfléchis, pour les feuillets externe et interne. On n’aura qu'à appliquer au feuillet interne ce qui sera dit dans un instant pour le feuillet externe. Pour le moment, on peut considérer ce feuillet interne comme une lamelle s'étendant de bas en haut, doublant pour ainsi dire la première, et s'allongeant d'avant en arrière par la soudure des.têtes des nouveaux rayons branchiaux qui se forment. Ainsi se trouve formé le feuillet réfléchi, qu'il serait mieux d'appeler, on le voit, le feuillet interne de la branchie interne; car il n'est pas le résultat d'une flexion, d'une réflexion de la fremière lamelle, mais bien d'un bourgeonnement de son bord inférieur, e'est-à-dire d'une production nouvelle et surajoutée.

\section{Branchie externe.}

Feuillet moyen direct ou descendant.

Pour déterminer le moment de l'apparition des différentes parties de l'appareil respiratoire, il faut trouver et prendre des points de repaire dans l'appareil lui-même. Ainsi nous avons déjà vu que le deuxième feuillet ne commenȩait à paraître que lorsque le premier portait près de douze rayons bien développés; la deuxième branchie ou, si l'on veut, le troisième feuillet, en allant de dedans en dehors, ne se montre que bien plus tard, e'est-à-dire quand les deux premiers, parfaitement constituès, ont déjà vingt rayons. A ce moment, la partie développée de l'appareil a tout à fait l'apparence d'une branchie, telle qu'elle se présente dans l'adulte, et le bord libre du feuillet interne, e'est-à-dire le bord supérieur, atteint presque le bord adhérent du feuillet direct ou moyen interne.

Alors les dissections deviennent indispensables, et elles sont bien plus laborieuses encore que lorsqu'il n'y avait qu'une seule lame.

On voit sur le bord externe de l'insertion du feuillet moyen interne, ou de la première lame directe de la branchie interne, vers son extrémité postérieure, naitre une série de tubercules, d'abord ¿̀ peine sensibles, mais qui bientôt sont séparés par des dépressious qui les éloignent, les limitent, et en forment de nouveaus filaments isolés comme ceux re la première lame an début de son 
développement. On voit leur multiplication avoir lien sur le bord exterue du cordon, résultat de la réunion des bases des filaments de la première rangée.

Les nouveaux filaments se développent d'abord (1) à l'extrémité postérieure de la branchie; ils s'étendent en avant ( 2 ) pour regagner l'extrémité antérieure de la première partie de l'appareil. Mais comme celle-ci continue toujours son développement en arrière, la nouvelle lame naissante se trouve bientôt dépassée par la première; aussi pour la suivre doit-elle s'étendre en arrière, de sorte qu'elle va s'allongeant par ses deux extrémités, en avant et en arrière.

Les bourgeons, du reste, prennent naissance, et s'étendent absolument comme dans les premiers cas; les plus anciens se trouvant an milieu (3) quand ils sont tout à fait transformés en rayons, on s'explique facilement la forme de cette lame branchiale (4) qui, large au milieu, cst rétrécie en pointe à ses deux extrémités.

Ces filaments commencent par être libres; ils ont une position à peu près parallèle avec ceux de l'autre lame, contre laquelle ils s'appliquent. Leur développement, leur forme, sont sensiblement les mêmes que pour la première lame dont nous avons donné la description en détail. Il n'y a done rien à ajouter. Les cils et les antres choses ont la même disposition.

Fenillet externe réflèchi ou ascendant.

Celui-ci parait quand le premier a déjà pris un certain développement (5); il en est ici encore comme du feuillet réfléchi de la première branchie ; aussi, quoique je n’aie point compté le nombre de rayons existant an moment où le feuillet qui nous occupe apparaît, je ne serais pas éloigné de penser que c’est quand il y a à peu près un nombre semblable à celui que présente la première lame directe,

(1) Voyez Zool., pl. 2, fig. $6\left(m^{\prime \prime}\right)$.

(2) Voyez Zool., pl. 2, fig. $6\left(m^{\prime}\right)$.

(3) Voyez Zool., pl. 2, fig. $6(\mathrm{~m})$.

(4) Voyez Zool., pl. 2, fig. $7(q-m)$.

(5) Voyez Zool., pl. 2, fig. 8. 
que commence i se développer le feuillet 'réfléchi. En tout cas, ce développement se passe absolument de la même manière que pour le feuillet réfléchi de la branchie interne. Les têtes des rayons prennent un accroissement considérable, se rapprochent, se soudent en dehors. La soudure semble former un pont (1) entre les têtes voisines, et bientôt une lamelle est produite ; celle-ci s'accroît forcément par ses deux extrémités, puisque la branchie, sur laquelle elle prend naissance, s'étend par les deux bouts; mais comme c'est surtout en arrière que la lame réfléchie a commencé son développement, les parties les mieux formées sont-elles tont près de l'extrémité postérieure (2).

Voilà comment naissent les lames, les feuillets réfléchis indirects ou ascendants.

Comment, maintenant, se forment sur eux les fentes branchiales? Il y a ici un mode de développement tout différent de celui que nous ont offert les lames directes; celles-ci ont commencé par n'être point des lames; elles ne le sont devenues que lorsque, par une soudure de leur extrémité, les rayons ont été fixés dans un même plan. Si l'on veut, les premiers feuillets ont commencé par être déchiquetés en lanières ; la soudure en a formé plus tard une lame; ici, au contraire, la lame commence par être pleine. Comment sera-1-elle divisée en bandelettes?

Les têtes des rayons (3) sont toujours comme terminées par trois lobes qui rappellent un trètle, dont les divisions seraient larges et empâtées. Les ponts de soudure s'établissent entre les lobes latéraux , tandis que les lobes médians font saillie sur le bord libre, en s'effaçant de plus en plus sur la face de la lame; il en résulte des ondulations, des dépressions et intervalles, qui correspondent aux espaces placés entre les rayons.

Dans ces points, on voit une dépression qui, d'alord ova-

(1) Voyez Zool., pl. 2, fig. $8(a, b, c)$.

(2) Voyez Zool., pl. 2, fig. $8(b)$.

(3) Voyez cette même figure 8 de cette planche 2. En suivant du sommet à la base, on peut reconnaitre tous les intermédiaires entre les premier's rayons à peine soudés et ceux où le pont est complet. 
laire et en continuité avec la dépression générale, s'élargit de plus en plus, et prend une transparence qui indique un amineissement de la lamelle; bientôt on distingue un véritable orifice, terminé en pointe aiguë du côté du bord libre ; des cils viennent en garnir le pourtour, et une fente se trouve ainsi formée par érosion sur la branchie externe, dont la lame directe a de dix-huit à vingt rayons (1). On voit tous les passages entre une fente branchiale bien nette et une simple dépression dans la lamelle qui n'est pas formée complétement partout, et qui se prolonge encore en naissant par voie de bourgeonnement, ainsi qu'il a été dit.

On le voit, il y a une grande différence entre ce qui a lieu dans les feuillets rétléchis et les feuillets directs.

Il ne faut pas croire que la lamelle réfléchie de la seconde branchie ne se forme que lorsque la première est bien développée; non, elle commence avant que les baguettes ou rayons soient très allongées $(2)$. Il y a, en effet, cette différence entre elle et la première, que dans celle-ci la partie réfléchie ne se développe que beaucoup plus tard lorsqu'un grand allongement des rayons s'est déjà produit, tandis qu'ici le nombre des rayons est déjà considérable; et leur longueur cependant très médiocre quand déjà leur tête se soude.

Si nous résumons ce que uous venons de voir, nous trouvons les quatre feuillets se succédant dans l'ordre suivant : Feuillet moyen interne, ou feuillet direct de la branchie interne; feuillet réfléchi interne de la première branchie; apparition du deuxième feuillet moyen externe ou feuillet direct de la seconde branchie; enfin quatrième feuillet, ou feuillet réfléchi externe.

En d'autres termes, d'abord la branchie interne, feuillet direct, puis feuillet réfléchi ; ensuite la branchie externe, feuillet direct, feuillet réflérhi.

Voilà l'ordre d'apparition des feuillets. Quant à la direction et au mode d'accroissement, on a vu que toujours les deux feuillets

(1) Voyez Zool., t. IV, pl. 7, fig. \& $\left\{a, b, c, c^{\prime}\right\}$.

(2) Voyez Zool., t. IV, pl. T $(m, q)$. 
moyens ou directs commençaient l'interne cu avant, l'exterue en arrière ; ce qui conduit ì l'extension de l'un d'avant en arrière, tandis que pour l'autre elle est d'arrière en avant. Toutefois ce mode d'accroissement pour ce dernier est limité aux premiers moments de l'apparition; car la première lame continuant à s'accroìtre d'avant en arrière, et celle-ci la suivant dans son développement, il en résulte qu'elle doit aussi s'étendre d'avant en arrière.

Les feuillets réfléchis naissent toujours dans le point le plus développé de la lame à laquelle ils appartiennent. Il devait en être forcément ainsi.

On voit même d'après cela quelle doit être la marche de leur' accroissement, puisque l'une a commencé en avant, l'autre en arrière; ce doit ètre en avant et en arrière que commencent les feuillets réfléchis internes et externes. Mais tandis que le premier va toujours en s'accroissant d'avant en arrière, le second s'étend de deux manières, d'avant en arrière et d'arrière en avant ; ce qui entraîne un développement analogue dans les feuillets rélléchis.

Il suffit, je pense, d'opposer ces résultats à ceux que M. Löven a fait connaître, pour voir que le savant malacologiste du Nord n'a pas suivi complétement la formation des organes de la respiration; que l'opinion hypothétique qu'il émet avec doute, il est vrai, ne s'accorde pas entièrement aver ce qui vient d'être indiqué ici.

Il dit, en effet : " On ne peut plus douter que ces grands organes „ ne soient des branchies, quoique je u'aie jamais pu me convaincre ") qu'il y en ait deux de chaque côté ; car je n'ai jamais distingué " qu'un senl pli. "Et plus loin : "On doit remarquer qu'il existe "des Mollusques qui n'ont qu'un feuillet de chaque còté, qui se " sépare en deux, peut-être longtemps après (1). " On esı, en effet, porté à croire à un dédoublement, à une division du feuillet branchial; mais quand on a suivi le développement, il n'y a plus de doute possible.

Il reste enfin à examiner si l'on doit considérer comme des branchies les deux lignes ciliées, parallèles aux bords de la coquille, que l'on trouve indiquées dans un Mémoire inséré dans

(1) Académie de Stockholm, 1848. Citation précédente. 
les Actes de la Société de biologie, et qui sont regardés par l'auteur comme les branchies des jeunes Huîtres.

Sans doute, je n'ai pas la prétention de dire que, dans tout le groupedes Acéphales lamellibranches, les branchies se développent comme dans la Moule. Je me hâte moins dans mes généralisations; mais quand je considère ce qui a été vu par M. Löven sur un grand nombre d'espèces, sur le Taret par M. de Quatrefages, par moi-même sur les Huitres, les Moules, les Cyclas, etc., je dois l'avouer, j'ai peine ì croire que la nature développe les branchies en partant de choses complétement différentes; et pendant que les savants que je viens de nommer, si habitués aux recherches d'embryogénie et d'anatomie comparées, trouvent une disposition constante, alors même qu'ils ne peuvent encore reconnaitre l'organe qui se développe, il me paraît impossible d'abandonner ma manière de voir, qui est en tout conforme avec ce qui a été vu, pour embrasser une opinion nouvelle, présentée par un homme dont les travaux ne semblent pas indiquer une grande habitude de l'anatomie comparée, surtout de celle des animaux inférieurs.

"Voir venir les clıoses, a dit Turpin, est le meilleur moyen de " les connaître. "Cela sera vrai, à la condition de les suivre jusqu'à ce qu'il n'y ait plus ancun doute sur leur nature. Or, je le demande, dan's les deux lignes ciliées, qui sont l'une parallèle au bord du mantean, l'autre voisine de la charnière, comment voir les rudiments des branchies? M. Davaine a pris tout simplement les cils vibratiles du bord du mantean pour l'une des branchies, et peut-être ceux de l'estomac pour l'autre.

Je sais hien qu'on me dira que les branchies de l'Huitre sont autrement construites que celles de la Moule; cela est vrai, mais l'origine de l'organe de la respiration est si éloignée, dans le Mémoire que je cite, de re qui a été vu par MM. Löven, de Quatrefages ot moi-même, qu’il me paraît impossible de ne pas trouver cela une grossière erreur.

D’ailleurs en embryogénie il ne suffit pas de dire : Ceci est tel organe qui eommenee! Ine assertion semblable r'a de valeur et ne devient positive que lorsqu'on l’a démontrés en suivant le développrement ultóriemr. Or ce n'est pas ce yui a été fait; dans ce même 
travail, les organes de la circulation sont aussi décrits, le nombre des pulsations du cœur est même compté, et il suffit de voir les lessins qui l'accompagnent, de les comparer ì cenx publiés non par moi, mais par M. Löven, pour reconnaitre la valeur de pareilles recherches, et pour voir que c'est à peine si elles méritent d'être prises au sérieux.

On a vu que, dans l'adulte, les filaments des branchies restaient rapprochés par l'enchevêtrement, l'intrication des cils vibratiles disposés en houppes, et mus de mouvements ondulatoires, qui ne prenaient aucune part ì la formation des courants. Ce mode d'union s’est présenté dans l'éturle du développement. J'ai vu, en effet, paraitre de loin en loin sur les jeunes branchies des tubercules, dont la transformation m'a montré ce que j’ai décrit en commençant. Ils se sont couverts d'un duvet d'une finesse extrême; les cils se sont peu à peu allongés, et agités de mouvements ondulatoires.

En face de chaque tubercule, il s'en développe un autre sur le rayon voisin, et les deux tuhercules, par le progrès dı développement, s'approchant de plus en plus, finissent par produire l'effet dont il est question, par mêler les cils qui les courrent.

Un examen rapide ferait croire à des travées tendues entre les rayons; mais, avec un grossissement suffisant, on ne tarde pas à s'apercevoir que le mode d'union est bien tel que je viens de l'indiquer.

Faut-il voir ici dans ces tubercules le commencement de ces bandes transversales, qui, dans l'Huitre et dans l'Anodonte, la Pholade, la Lutraire, etc., transforment les lames branchiales en un réseau à mailles quadrilatères? S’il en était ainsi, la natıre s'arrêterait en route, et au lieu d'un filament transversal pleiı, il n’y aurait que les tubercules ciliés formant une union qui peut être interrompue momentanément, et se rétablir ensuite.

Il faut ajouter que dans la branchie des Moules on trouve rependant de loin en loin de véritables baguettes tamsversales assez. longues qui unissent les rayons, et consolident ainsi les lames, dont les éléments sont si facilement dissociables. 
Quant aux eils vibratiles, ils sont de plusieurs sortes sur la branchie arrivée à son entier développement. Sur chaque rayon, ils forment quatre séries régulières, linéaires, placées deux en dedans, deux en dehors, sur les côtés antérieur et postérieur. Si l'on se représentait ces rayons comme étant prismatiques, lleux des faces étant dirigées, l'une en avant et l'autre en arrière, les rangées des cils occuperaient exactement les arêtes; ce sont là les cils de la respiration, et qui agitent et renouvellent l'eau ì la surface de l'organe.

Mais il en est d'une autre espèce et plus rares, placées de loin en loin, isolément, plus longs, dont les mouvements sont moins rapides et moins réguliers, et qui semblent destinés à balayer la surface des branchies, à la débarrasser des petits corpuscules qui resteraient mêlés aux rayons, bien plutòt qu’ils ne paraissent destinés à agiter l'eau.

Plus tard entin, une nouvelle rangée de cils très fins vient se placer ì côté des quatre premières et en dedans d'elles, non pas par rapport à l'axe de l'animal, mais par rapport à l'axe même dı rayon. Ceux-ci ne paraissent pas prendre un accroissement considérable; ils restent sous forme d'un fin duvet.

La structure de l'organe mériterait une étude altentive. En partant de celle que présente l'adulte, j'en indiquerai les traits principatix.

On trouve au centre des rayons un tube résistant qui forme romme une charpente, plus solide, plus forte, qui semble correspondre amx vaisseaux: Quand on fait les préparations nécessaires pour l'étude, la partie qui entoure cette charpente est eulevée sur III grand nombre de points, car elle est cellulaire, et facile ì déchirer. On a souvent le tube central isolé entouré encore dans quelques points par le tissu dont je parle, qui porte les cellules épithéliales à cils vihratiles.

Mais je dois signaler ici une lacune. A quel moment se forment les vaisseaux de la branchie? C'est là une chose que je ne pourrais dire. Jamais je n'ai vu de mouvement dans les jeunes hranchies, même dans celles qui avaient déjà quatre feuillets. L'apparition lardive du cœur expliquerait peut-être cela; cepen- 
lant, il faut le dire, pour prendre une détermination, un doit être plein de réserve, car les préparations sont d'une telle délicatesse que l'on pourrait bien facilement faire erreur. M. Löven luimème se borne à quelques mots, dans lesquels il déclare n'avoir jamais été assez heureux pour voir naître le cœur. Ce qu'on peu affirmer, e'est que l'organe de la respiration existe déjà avant le ccur, et que l'un précède l'autre. Je parle toujours de la Youle.

Si, dans l'Huitre, les choses se passent comme dans la Moule, on ne comprend pas comment M. Davaine a pu étudier le cœur; il est évident qu'il n'a pas vu les branchies; et dès lors il semble difficile que le prétendu cœur, dont il a cependant compté avec soin les pulsations, puisqu'il en fixe le nombre par minute, soit bien le véritable organe central de la circulation. Il est probable qu'un bouquet de poils vibratiles a été pris pour le copur sur queluque animal rentré dans sa petite coquille.

v.

CONCLUSION.

Je crois, ainsi que je l'annonçai, avoir pris un organe à son origine, en avoir suivi toutes les modilieations, et ètre arrivé à la forme qu'il a dans l'animal parfait; c'est done plus que de l'embryogénie générale, c'est l'embryogénie particulière d'un organe que je me proposais de faire. Me serais-je trompé en croyant avoir atteint le but?

Mais je n'ai fail, je n'ai pu faire l'étude du développenent des branchies que d'un seul animal; je n'ai done point de comparaison à établir, puisque les éléments manqueraient pour cela. Cependant il y a des rapprochements qui ne peurent être passés sous silence, et il me semble même que, sans trop se hasarder, on peut cherrher à se faire, à priori, une idée générale de quelques dispositions principales de l'organe respiratoire dans les Acéphales lamellibranches, sauf à les confirmer ou les abandonuer ensuite à posteriori.

L.es bramchies présentent des différences qui tienuent à la sout- 
dure des lames réfléchies, et au plus ou moins grand développement de l'une des deux.

Ainsi, dans les Huitres, les Anodontes, les Pholades, les Li1traires, et tousles Acéphales (1) qui vivent plus ou moins ensablés ou enfermés, on trouve que les feuillets réfléchis externes sont soudés avec le manteau par les bords que nous avons vı être libres dans la Moule; que les feuillets réfléchis internes sont soudés entre eux par leur bord supérieur; de telle sorte qu'en ouvrant ces animaux, les branchies apparaissent comme une lame plusieurs fois ployée sur elle-même. Mais en y regardant de près, en cherehant surtout à disséquer, on ne tarde pas à trouver les traces des soudures. C'est ainsi que dans les Cardium, les bords des feuillets réfléchis internes sont soudés en partie entre eux, en partie avec la masse viscérale. Les plus légères tractions détruisent ces soudures; ainsi que dans beaucoup d'autres exemples, les Mactres, les Vénus, etc., où une disposition tout à fait analogue se présente.

Il était nécessaire pour ces animaux que l'eau, entrant par un des tubes, dùt forcément traverser le grillage branchial pour sortir par l'autre; ainsi se trouvait assuré l'acconplissement de l'acte de la respiration.

En trouvant dans celte disposition, si différente en apparence, une même chose, on peut croire à un développement semblable à celui qui vient d'être indiqué. Les soudures ne fourniraient point des raisons suffisantes pour écarter une telle opinion; car li facilité avec laquelle on látache les bords internes et externes des branchies de l'Anomie, do la Bucarde, etc., prouverait assez que ces soudures, au moins daus re cas, ne se sont effectuées qu'après ce développement. Qu'on crsaie, an contraire, d'enlever les lames directes, et l'on verra quellr différence il y a dans la inanière dont s'aecomplit la séparation dans les deux eas.

Mais il lant convenir 'pue daus l'Hnître, les Anodontes, les Lnio, etr., il y a me soudure bien autrement solide et résistante.

(1) Je renvoie pour la détermination des espéces, dont je donne seulement les noms français, à un travail que j'ai publié sur les Acéphales lamellibranches, et ou j'ai soigneusement indiqué la spécification. (. $4 \mathrm{mn}$ des $s c . n a l ., \mathrm{t}$. II, $4^{\mathrm{e}}$ série, p. $1: ; \%)$ 
Cependant je crois qu'ici encore les soudures doivent s'effectuer secondairement, et, sans pouvoir affirmer que le développement suive une marche identiquement la même, je ne puis m'empêcher d'admettre qu'il se fait d'après un plan général, à peu près le même aux détails près. Il y a évidemment bien des particularités qui doivent encore être étudiées, et qui nous feront connaitre comment se forment tous ces filaments transverses, qui transforment la branchie en un véritable grillage à mailles quadrilatères; mais ces détails ne suffisent pas pour faire supposer un plan complétement différent.

Si donc on admet une même marche dans le développement des branchies, on s'expliquera très nettement et très simplement quelques variétés de forme et de disposition del'organe de la respiration.

Ainsi, dans quelques cas, on ne trouve qu'une seule branchie de chaque côté ; dans d'autres, on en trouve bien deux, mais l'une d'elles est très petite, relativement à l'autre qui est très développée.

Comment expliquer cette absence de l'une, cette inégalité de l'autre?

On a vu que la seconde branchie ne paraissait que lorsque la première était déjà développée, et que son feuillet réfléchi atteignait presque le point de départ de la lame directe.

Si donc le développement s'arrête en chemin, la seconde branchie aura une moins grande largeur que la première, puisqu'elle est arrivée plus tard.

On se rappelle aussi que cette même seconde branchie doit s'accroìtre d'arrière en avant, puisqu'elle commence à l'extrémité postérieure de la première; donc, si le développement s'arrête, celle-ci devra être plus courte, moins longue que la première.

La mème raison peut expliquer comment la hauteur est moindre.

A un certain moment (1), labranchie de la Moule doit done représenter la disposition des Mollusques auxquels je fais allusion. Dans beaucoup de Cardium, on sait combien est courte la deuxième branchie ou branchie externe, de même dans la Cardita sulcata,

(1) Voy. Zool., pl. 2, fig. 7. Cette figure peut donner une idée de la comparaison que je veux établir; mais il faut dire que la branchie externe $(m, g)$, plus développée, serait plus rapprochée de la vérité. 
la Petricola ruperella, la Chama gryphö̈des. Dans la Pandora rostrata, on croirait au premier abord que la branchie externe, ressemblant à un bourrelet, est formée d'un seul feuillet, tant son développement est peu considérable. Ainsi un arrêt de développement suffit pour expliquer cette disposition. Quand la première branchie de la Moule est bien développée, et que la seconde commence à avoir ses deux feuillets, on a tout à fait l'apparence des branchies irrégulières.

Enfin il est des espèces auxquelles il manque une branchie de chaque côte; les Lucines sont dans ce cas. Il était curieux et intéressant de reconnaître laquelle des deux branchies manquait ; était-ce celle qui se développe la première ou celle qui apparaît la seconde? En examinant les Lucines (Lucina lacta) de l'étang de Thau, j'ai bientôt trouve que la branchie unique portait un repli en dedans, et que par conséquent elle devait être la branchie interne, c'est-àdire la première développée. Ici l'arrêt de développenent ne porterait pas seulement sur l'étendue de la branchie externe, mais sur la totalité de cette portion de l'organe.

Ici encore, à un certain moment, la branchie de la lloule rappelle tout à fait la branchie de la Lucine (1); c'est lorsque les deux premières lames sont seules développées, avant que les secondes paraissent.

Ces exemples suffisent, je crois, pour montrer que le développement doit se faire d'après un même plan daus les Acéphales, et pour prouver surtout combien l'embryogénie est utile et nécessaire, dans l'appréciation des formes, qui, sans elle, ne peuvent être rapportées à rien de précis; elle nous sert à montrer l'unité de plan de composition, principe si fécond, quand il est convenablement appliqué dans les groupes dı règne animal, où sou existence ne saurait être mise en doute.

\section{EXPLICATION DES FIGURES.}

PLANCHE ๖.

Fig. 1. Petite Moule, plus avancée que la suivante, vue de face, du côté des bords libres des valves, montrant la soudure du manteau $(m)$ et la languette (1) Voy. pl. 2, fig. 6. La branchie interne, débarrassée des tubercules $m, m^{\prime}, m^{\prime \prime}$, représente tout à fail celle d'une Lucine adulte. 
qui la termine en arrière; les branchies qui forment une cage, ressemblant au thorax $(b r)$; le pied $(p)$ couvert de cils vibratiles, et parcouru par une fente longitudinale; le byssus $(b)$.

Fig. 2. La même vue par le dos; elle est contractée. (ch, ch) charnière dentelée, $(l)$ ligament élastique, $(f)$ foie. $(g)$ estomac dans lequel paraissent les granulations rejetées au centre par les mouvements des cils vibratiles de la paroi. On distingue très nettement les deux lobes du foie; celui de gauche, plus étendu, semble bilobé.

Fig. 3. Jeune Moule, vue de profil par le côté droit. $(p)$ pied, $(f)$ foie, $(g)$ estomac. L'intestin, qui parait du côté de la charnière, se termine en arrière et en dessous du muscle postérieur des valves, que l'on voit en arrière de la vésicule transparente $(r)$; celle-ci doit être considérée avec M. Löven comme l'origine du corps de Bojanus. Trois rayons branchiaux sont complets $(c, b)$; l'un nouveau $(a)$, qui formera le quatrième, est séparé en avant des trois autres, et commence à s'isoler en arrière.

Fig. 4. Une Moule un peu plus avancée, vue sur le côté gauche. Mêmes lettres désignant mêmes choses. On y voit de plus $(o)$ les otolithes, et un paquet musculaire rétracteur du pied qui se rend auprès du muscle postérieur des valves en croisant le corps de Bojanus $(r)$. Le rayon $(a)$, qui, dans la figure précédente, est à peine saillant, ici au contraire est bien détaché. Les têtes de tous les rayons commencent à devenir trilobées.

Fig. 5. Branchie interne; feuillet moyen direct, de gauche, vu par le côtè interne en $(a)$. Les nouveaux rayons paraissent comme des bourgeons; c'est par cette extrémité postérieure que la branchie s'accroît. - Cette figure montre aussi le développement du feuillet réfléchi, qui nait par l'accolement et la soudure des têtes des rayons encore libres en $(a)$, mais rapprochés en $(b)$ et soudés en $\left(b^{\prime}\right)$. Le pont qui a été jeté entre les têtes, et dont on peut suivre les progrès de l'extrémité postérieure à l'extrémité antérieure, forme déjà une véritable lamelle en $c$.

Fig. 6. Branchie gauche, interne, vue par son côté externe : elle montre le développement très grand du feuillet rétléchi interne $(k)$, et le commencement de la seconde branchie $(m)$ qui bourgeonne en $\left(m^{\prime}\right)$ et en $\left(m^{\prime \prime}\right)$. Cette rangée de tubercules commence en arrière, et sur le cordon $(n)$ qui représente la base d'insertion des rayons de la première lame.

Fig. 7. La même branchie plus avancée en développement. La deuxième, qui ne paraissait qu'à l'état de rudiment dans la figure précédente, est ici déjà constituée; elle a un feuillet direct $(m)$ et un feuillet réfléchi $(q)$.

Fig. 8. Deuxième branchie séparée de la première, et plus fortement grossie, montrant l'origine des fentes branchiales de la lame réfléchie; c'est la branchie gauche externe. On voit que en $(a)$ il n'y a pas encore d'orifice, mais que en $(b)$ déjả des fentes bordées de cils vibratiles sont complétement percées. On les voit de moins en moins grandes à mesure que l'on s'avance davantage du 
côté de $(c)$ et en $(e)$. Le pont jeté en forme de lamelle, entre les têtes de rayons, est de moins en moins net, et même la soudure finit par étre quelque peu confuse.

Fig. 9. Tubercules qui se développent sur les faces des rayons, et qui enchevetrant les touffes de cils qu'ils portent, maintiennent les filaments en rapport les uns avec les autres. 

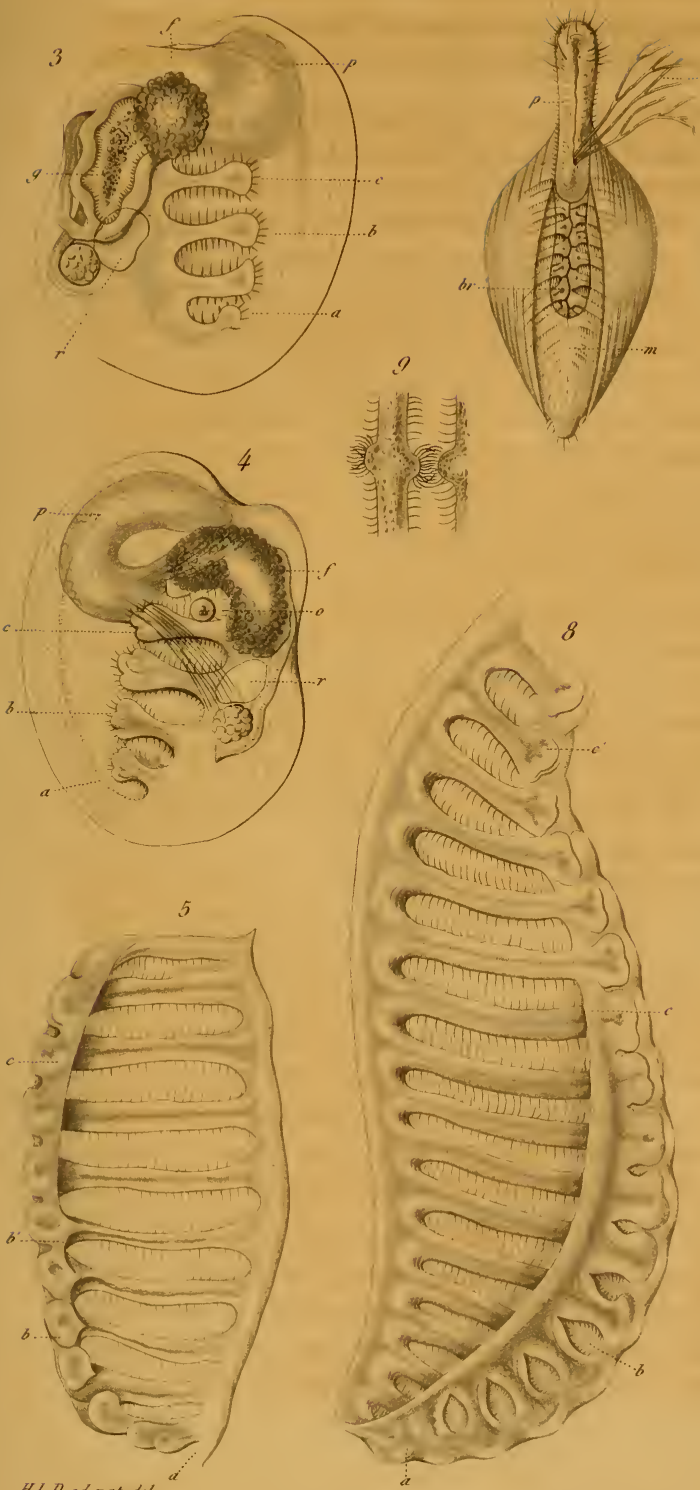

H.L.D. ad nat. atel. "

Déneloppement des Branchici.
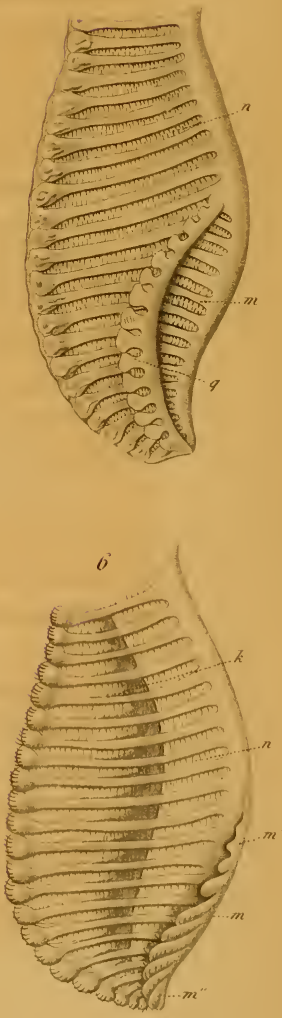

imyard si 



\section{TA B LE.}

Mémoire sur l'organisation de l'Anomie. . . . . $\quad \begin{array}{ccc}\text { Pages. } & \text { Planches. } \\ 1 & 1,2\end{array}$

Mémoire sur les organes génitaux des Mollusques acéphales lamellibranches. . . . . . . . . . . $33 \quad 5,6,7,8$

Mémoire sur l'organe de Bojanus. . . . . . . $127 \quad 4,5,6$

Mémoire sur le développement des branchies des Mollusques acéphales lamellibranches. . . . . . . . $181 \quad 2$ 







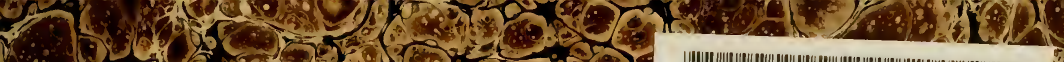

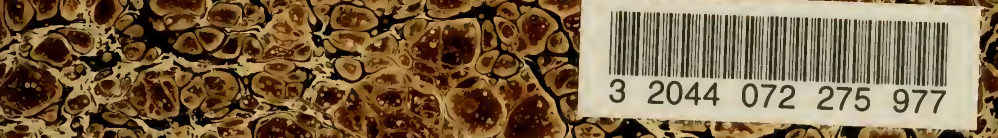

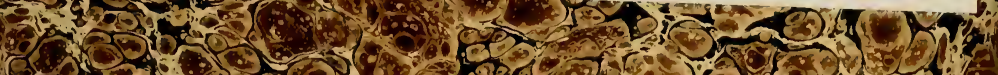

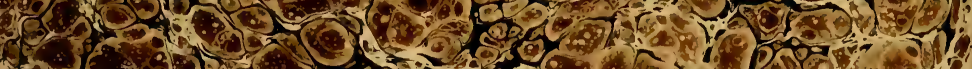

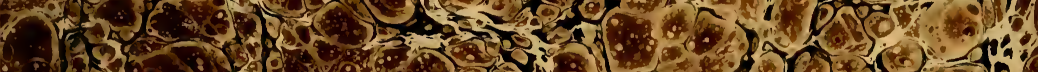
$(6) \times$

\section{6.}

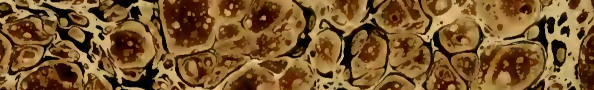

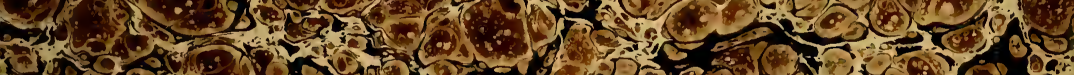

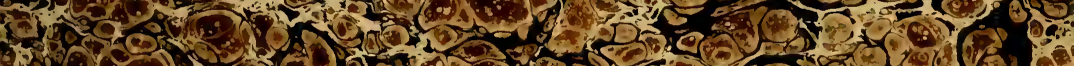
- Q

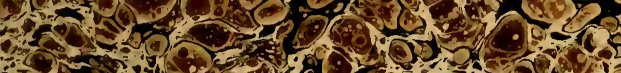

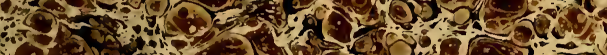

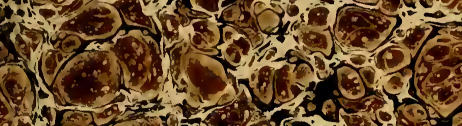

30

1. (.)

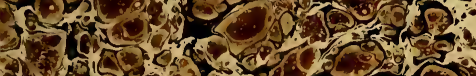

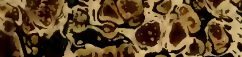

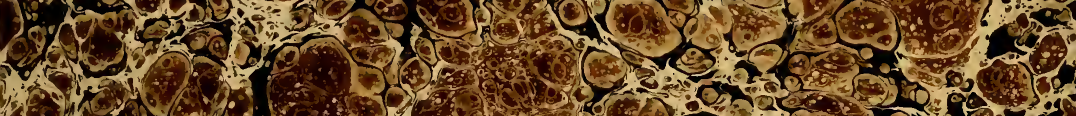
inger. 46. 20. 2.

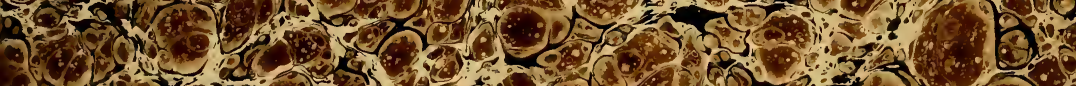
(1) 2. 2010 10.1. 10.5 1. -

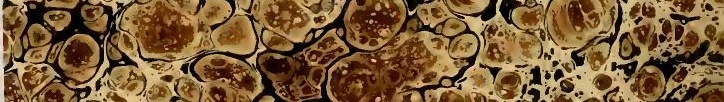

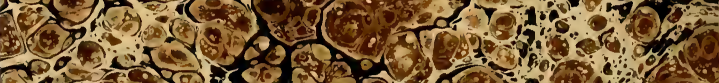

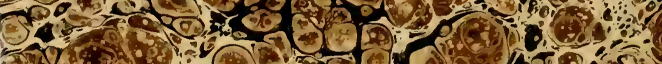
1 - O J H.C. 102. o or.

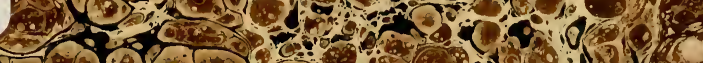




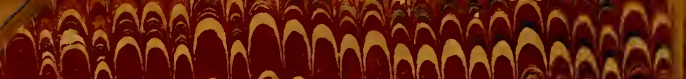

- An 10

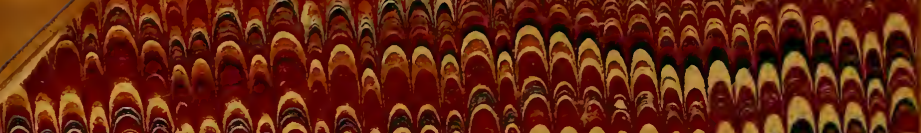
ranW A MAAAก AA A A A A

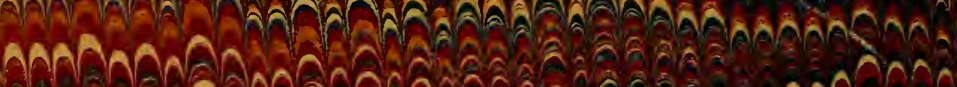
Maphwa

ล AลA

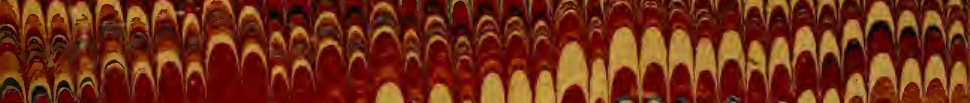

Ba

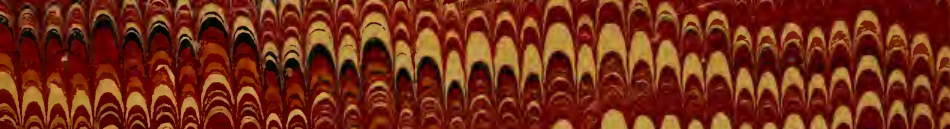
AMAN

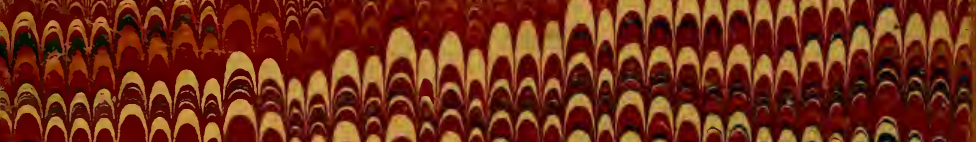
A Aam? A A*AAA

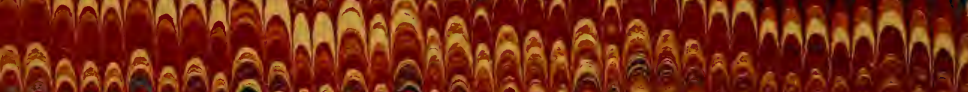
ANAAA A A A A A A ล O

S201 man

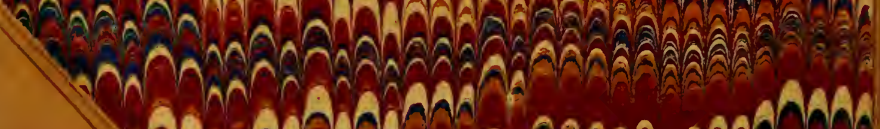

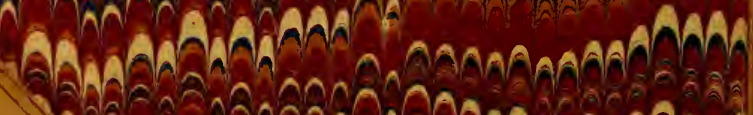
AMMAMAMAAN 